\title{
The Solar Wind as a Turbulence Laboratory
}

\author{
Roberto Bruno \\ Istituto Fisica Spazio Interplanetario - INAF, \\ Via Fosso del Cavaliere 100, 00133 Rome, Italy \\ email: bruno@ifsi.rm.cnr.it \\ http://www.ifsi.rm.cnr.it \\ and \\ Vincenzo Carbone \\ Dipartimento di Fisica, Università della Calabria \\ Ponte P. Bucci, Cubo 31C 87036 Rende (CS), Italy \\ email: carbone@fis.unical.it \\ http://fis.unical.it \\ Accepted on 9 April 2005 \\ Published on 20 September 2005 \\ http://www.livingreviews.org/lrsp-2005-4 \\ Living Reviews in Solar Physics \\ Published by the Max Planck Institute for Solar System Research \\ Germany
}

\begin{abstract}
In this review we will focus on a topic of fundamental importance for both plasma physics and astrophysics, namely the occurrence of large-amplitude low-frequency fluctuations of the fields that describe the plasma state. This subject will be treated within the context of the expanding solar wind and the most meaningful advances in this research field will be reported emphasizing the results obtained in the past decade or so. As a matter of fact, Ulysses' high latitude observations and new numerical approaches to the problem, based on the dynamics of complex systems, brought new important insights which helped to better understand how turbulent fluctuations behave in the solar wind. In particular, numerical simulations within the realm of magnetohydrodynamic (MHD) turbulence theory unraveled what kind of physical mechanisms are at the basis of turbulence generation and energy transfer across the spectral domain of the fluctuations. In other words, the advances reached in these past years in the investigation of solar wind turbulence now offer a rather complete picture of the phenomenological aspect of the problem to be tentatively presented in a rather organic way.
\end{abstract}

(C) Max Planck Society and the authors.

Further information on copyright is given at http://solarphysics.livingreviews.org/About/copyright.html

For permission to reproduce the article please contact info@solarphysics.livingreviews.org. 


\section{How to cite this article}

Owing to the fact that a Living Reviews article can evolve over time, we recommend to cite the article as follows:

Roberto Bruno and Vincenzo Carbone,

"The Solar Wind as a Turbulence Laboratory",

Living Rev. Solar Phys., 2, (2005), 4. [Online Article]: cited [<date $>$ ], http://www.livingreviews.org/lrsp-2005-4

The date given as $<$ date $>$ then uniquely identifies the version of the article you are referring to.

\section{Article Revisions}

Living Reviews supports two different ways to keep its articles up-to-date:

Fast-track revision A fast-track revision provides the author with the opportunity to add short notices of current research results, trends and developments, or important publications to the article. A fast-track revision is refereed by the responsible subject editor. If an article has undergone a fast-track revision, a summary of changes will be listed here.

Major update A major update will include substantial changes and additions and is subject to full external refereeing. It is published with a new publication number.

For detailed documentation of an article's evolution, please refer always to the history document of the article's online version at http://www. livingreviews.org/lrsp-2005-4. 


\section{Contents}

1 Introduction $\quad \mathbf{5}$

1.1 What does turbulence stand for? . . . . . . . . . . . . . . . . . . . 6

1.2 Dynamics vs. statistics . . . . . . . . . . . . . . . . . . . 10

2 Equations and Phenomenology $r$

2.1 The Navier-Stokes equation and the Reynolds number . . . . . . . . . . . . . . . . 14

2.2 The coupling between a charged fluid and the magnetic field . . . . . . . . . . . . 15

2.3 Scaling features of the equations . . . . . . . . . . . . . . . . . . . 16

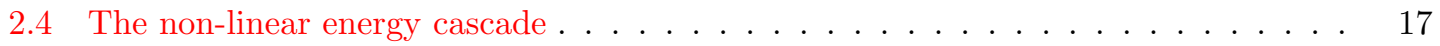

2.5 The inhomogeneous case . . . . . . . . . . . . . . . . . . . . 19

2.6 Dynamical system approach to turbulence . . . . . . . . . . . . . . . . . . . 19

2.7 Shell models for turbulence cascade . . . . . . . . . . . . . . . . . . . . . . . . 22

2.8 The phenomenology of fully developed turbulence: Fluid-like case . . . . . . . . . . 23

2.9 The phenomenology of fully developed turbulence: Magnetically-dominated case . 24

2.10 Some exact relationships . . . . . . . . . . . . . . . . . . . 25

3 Early Observations of MHD Turbulence in the Ecliptic $\quad 27$

3.1 Turbulence in the ecliptic . . . . . . . . . . . . . . . . . . . . . . . . 27

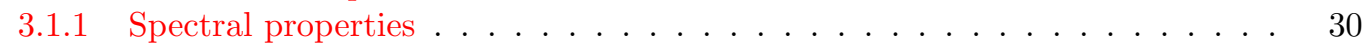

3.1.2 Evidence for non-linear interactions . . . . . . . . . . . . . . . . . . . . . . . . . . . . . . 33

3.1.3 Fluctuations anisotropy . . . . . . . . . . . . . . . . . . . . . . . . . . . . . . . . 35

3.1.4 Simulations of anisotropic MHD . . . . . . . . . . . . . . . . . . . . . . . . . . . . . . . . . 37

3.1.5 Fluctuations correlation length and the Maltese Cross . . . . . . . . . . . 38

3.1 .6 Magnetic helicity . . . . . . . . . . . . . . . . . . . . 42

3.1.7 Alfvénic correlations as uncompressive turbulence . . . . . . . . . . . 45

3.1.8 Radial evolution of Alfvénic turbulence . . . . . . . . . . . . . . . . . . . . . 47

3.2 Turbulence studied via Elsässer variables . . . . . . . . . . . . . . . . . . 51

3.2 .1 Ecliptic scenario . . . . . . . . . . . . . . . . . 51

3.2.2 On the nature of Alfvénic fluctuations . . . . . . . . . . . . . . 55

4 Observations of MHD Turbulence in the Polar Wind $\quad 60$

4.1 Evolving turbulence in the polar wind . . . . . . . . . . . . . . . . . 60

4.2 Polar turbulence studied via Elsässer variables . . . . . . . . . . . . . . . . . . 72

5 Numerical Simulations $\quad \mathbf{7 7}$

5.1 Local production of Alfvénic turbulence in the ecliptic . . . . . . . . . . . . . . 78

5.2 Local production of Alfvénic turbulence at high latitude . . . . . . . . . . . . 80

6 Compressive Turbulence $\quad \mathbf{8 3}$

6.1 On the nature of compressive Turbulence . . . . . . . . . . . . . . . . 85

6.2 Compressive turbulence in the polar wind . . . . . . . . . . . . . . . . . 89

6.3 The effect of compressive phenomena on Alfvénic correlations . . . . . . . . . . 93

7 A Natural Wind Tunnel $\quad 95$

7.1 Scaling exponents of structure functions . . . . . . . . . . . . . . . . . 95

7.2 Probability density functions and self-similarity of fluctuations . . . . . . . . . 99

7.3 What is intermittent in the solar wind turbulence? The multifractal approach . . . 100

7.4 Fragmentation models for the energy transfer rate . . . . . . . . . . . . . 105

7.5 A model for the departure from self-similarity . . . . . . . . . . . . . . . . 106 
7.6 Intermittency properties recovered via a shell model . . . . . . . . . . . . . . 106

8 Intermittency Properties in the 3D Heliosphere: Taking a Look at the Data 111

8.1 Structure functions . . . . . . . . . . . . . . . . . . . 111

8.2 Probability distribution functions . . . . . . . . . . . . . . . . . . 113

9 Turbulent Structures $\quad 117$

9.1 Radial evolution of intermittency in the ecliptic . . . . . . . . . . . . . . . . . 124

9.2 Radial evolution of intermittency at high latitude . . . . . . . . . . . . . 129

10 Conclusions and Remarks 132

11 Acknowledgments $\quad 134$

12 Appendix A: Some Characteristic Solar Wind Parameters 135

13 Appendix B: Tools to Analyze MHD Turbulence in Space Plasmas 136

13.1 Statistical description of MHD turbulence . . . . . . . . . . . . . . . . . . 137

13.2 Spectra of the invariants in homogeneous turbulence . . . . . . . . . . . . . . 139

13.2 .1 Coherence and phase . . . . . . . . . . . . . . . . . . . . 141

13.3 Introducing the Elsässer variables . . . . . . . . . . . . . . . . . . . . 141

13.3.1 Definitions and conservation laws . . . . . . . . . . . . . . . . . . . . 142

13.3.2 Spectral analysis using Elsässer variables . . . . . . . . . . . . . . . 143

14 Appendix C: Wavelets as a Tool to Study Intermittency 144

15 Appendix D: Reference Systems $\quad 147$

15.1 Minimum variance reference system _ . . . . . . . . . . . . . . . . . . 148

15.2 The mean field reference system . . . . . . . . . . . . . . . . . . . . 149

16 Appendix E: On-board Plasma and Magnetic Field Instrumentation 151

16.1 Plasma instrument: The top-hat . . . . . . . . . . . . . . . . . . 151

16.2 Measuring the velocity distribution function . . . . . . . . . . . . . . . . 152

16.3 Computing the moments of the velocity distribution function . . . . . . . . . 153

16.4 Field instrument: The flux-gate magnetometer . . . . . . . . . . . . . . . . 154

17 Appendix F: Spacecraft and Datasets 159

$\begin{array}{ll}\text { References } & 185\end{array}$ 


\section{Introduction}

The whole heliosphere is permeated by the solar wind, a supersonic and super-Alfvénic plasma flow of solar origin which continuously expands into the heliosphere. This medium offers the best opportunity to study directly collisionless plasma phenomena, mainly at low frequencies where high-amplitude fluctuations have been observed. During its expansion, the solar wind develops a strong turbulent character, which evolves towards a state that resembles the well known hydrodynamic turbulence described by Kolmogorov (1941). Because of the presence of a strong magnetic field carried by the wind, low-frequency fluctuations in the solar wind are usually described within a magnetohydrodynamic (MHD, hereafter) benchmark (Kraichnan, 1965; Biskamp, 1993; Tu and Marsch, 1995a; Biskamp, 2003). However, due to some peculiar characteristics, the solar wind turbulence contains some features hardly classified within a general theoretical framework.

Turbulence in the solar heliosphere plays a relevant role in several aspects of plasma behavior in space, such as solar wind generation, high-energy particles acceleration, plasma heating, and cosmic rays propagation. In the 1970s and 80s, impressive advances have been made in the knowledge of turbulent phenomena in the solar wind. However, at that time, spacecraft observations were limited by a small latitudinal excursion around the solar equator and, in practice, only a thin slice above and below the equatorial plane was accessible, i.e., a sort of $2 \mathrm{D}$ heliosphere. A rather exhaustive survey of the most important results based on in-situ observations in the ecliptic plane has been provided in an excellent review by Tu and Marsch (1995a) and we invite the reader to refer to that paper. This one, to our knowledge, has been the last large review we find in literature related to turbulence observations in the ecliptic.

In the 1990s, with the launch of the Ulysses spacecraft, investigations have been extended to the high-latitude regions of the heliosphere, allowing us to characterize and study how turbulence evolves in the polar regions. An overview of Ulysses results about polar turbulence can also be found in Horbury and Tsurutani (2001). With this new laboratory, relevant advances have been made. One of the main goals of the present work will be that of reviewing observations and theoretical efforts made to understand the near-equatorial and polar turbulence in order to provide the reader with a rather complete view of the low-frequency turbulence phenomenon in the 3D heliosphere.

New interesting insights in the theory of turbulence derive from the point of view which considers a turbulent flow as a complex system, a sort of benchmark for the theory of dynamical systems. The theory of chaos received the fundamental impulse just through the theory of turbulence developed by Ruelle and Takens (1971) who, criticizing the old theory of Landau and Lifshitz (1971), were able to put the numerical investigation by Lorenz (1963) in a mathematical framework. Gollub and Swinney (1975) set up accurate experiments on rotating fluids confirming the point of view of Ruelle and Takens (1971) who showed that a strange attractor in the phase space of the system is the best model for the birth of turbulence. This gave a strong impulse to the investigation of the phenomenology of turbulence from the point of view of dynamical systems (Bohr et al., 1998). For example, the criticism by Landau leading to the investigation of intermittency in fully developed turbulence was worked out through some phenomenological models for the energy cascade (cf. Frisch, 1995). Recently, turbulence in the solar wind has been used as a big wind tunnel to investigate scaling laws of turbulent fluctuations, multifractals models, etc. The review by Tu and Marsch (1995a) contains a brief introduction to this important argument, which was being developed at that time relatively to the solar wind (Burlaga, 1993; Carbone, 1993; Biskamp, 1993, 2003; Burlaga, 1995). The reader can convince himself that, because of the wide range of scales excited, space plasma can be seen as a very big laboratory where fully developed turbulence can be investigated not only per se, rather as far as basic theoretical aspects are concerned.

Turbulence is perhaps the most beautiful unsolved problem of classical physics, the approaches used so far in understanding, describing, and modeling turbulence are very interesting even from a historic point of view, as it clearly appears when reading, for example, the book by Frisch (1995). 
History of turbulence in interplanetary space is, perhaps, even more interesting since its knowledge proceeds together with the human conquest of space. Thus, whenever appropriate, we will also introduce some historical references to show the way particular problems related to turbulence have been faced in time, both theoretically and technologically. Finally, since turbulence is a phenomenon visible everywhere in nature, it will be interesting to compare some experimental and theoretical aspects among different turbulent media in order to assess specific features which might be universal, not limited only to turbulence in space plasmas. In particular, we will compare results obtained in interplanetary space with results obtained from ordinary fluid flows on Earth, and from experiments on magnetic turbulence in laboratory plasmas designed for thermonuclear fusion.

\subsection{What does turbulence stand for?}

The word turbulent is used in the everyday experience to indicate something which is not regular. In Latin the word turba means something confusing or something which does not follow an ordered plan. A turbulent boy, in all Italian schools, is a young fellow who rebels against ordered schemes. Following the same line, the behavior of a flow which rebels against the deterministic rules of classical dynamics is called turbulent. Even the opposite, namely a laminar motion, derives from the Latin word lámina, which means stream or sheet, and gives the idea of a regular streaming motion. Anyhow, even without the aid of a laboratory experiment and a Latin dictionary, we experience turbulence every day. It is relatively easy to observe turbulence and, in some sense, we generally do not pay much attention to it (apart when, sitting in an airplane, a nice lady asks us to fasten our seat belts during the flight because we are approaching some turbulence!). Turbulence appears everywhere when the velocity of the flow is high enough ${ }^{1}$, for example, when a flow encounters an obstacle (cf. e.g., Figures 1 and 2) in the atmospheric flow, or during the circulation of blood, etc. Even charged fluids (plasma) can become turbulent. For example, laboratory plasmas are often in a turbulent state, as well as natural plasmas like the outer regions of stars. Living near a star, we have a big chance to directly investigate the turbulent motion inside the flow which originates from the Sun, namely the solar wind. This will be the main topic of the present review.

Turbulence that we observe in fluid flows appears as a very complicated state of motion, and at a first sight it looks (apparently!) strongly irregular and chaotic, both in space and time. The only dynamical rule seems to be the impossibility to predict any future state of the motion. However, it is interesting to recognize the fact that, when we take a picture of a turbulent flow at a given time, we see the presence of a lot of different turbulent structures of all sizes which are actively present during the motion. The presence of these structures was well recognized long time ago, as testified by the beautiful pictures of vortices observed and reproduced by the Italian genius L. da Vinci, as reported in the textbook by Frisch (1995). Figure 3 shows, as an example, one picture from Leonardo which can be compared with Figure 4 taken from a typical experiment on a turbulent jet.

Turbulent features can be recognized even in natural turbulent systems like, for example, the atmosphere of Jupiter (see Figure 5). A different example of turbulence in plasmas is reported in Figure 6 where we show the result of a typical high resolution numerical simulations of 2D MHD turbulence. In this case the turbulent field represents the current density. These basic features of mixing between order and chaos make the investigation of properties of turbulence terribly complicated, although extraordinarily fascinating.

When we look at a flow at two different times, we can observe that the general aspect of the flow has not changed appreciably, say vortices are present all the time but the flow in each single point of the fluid looks different. We recognize that the gross features of the flow are reproducible

\footnotetext{
${ }^{1}$ This concept will be explained better in the next sections.
}

Living Reviews in Solar Physics

http://www . livingreviews . org/Irsp-2005-4 


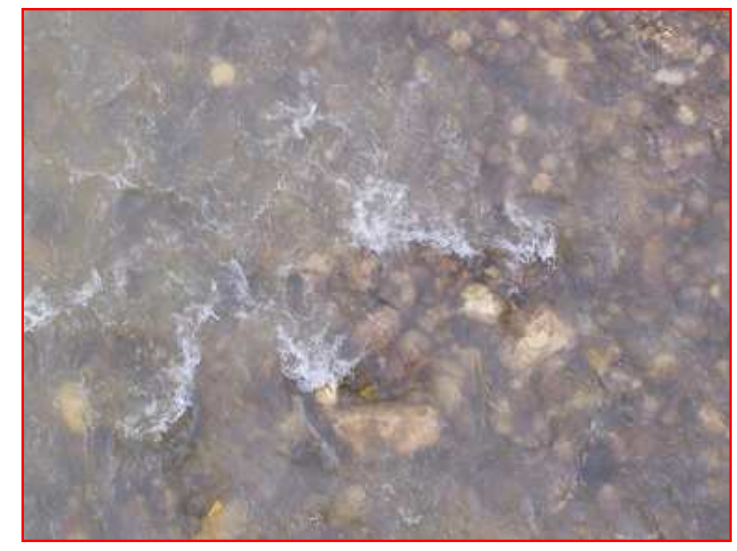

Figure 1: Turbulence as observed in a river. Here we can see different turbulent wakes due to different obstacles (simple stones) emerging naturally above the water level. The photo has been taken by the authors below the dramatically famous Crooked Bridge in Mostar (BosniaHercegovina), which was destroyed during the last Balcanic war, and recently re-built by Italian people.

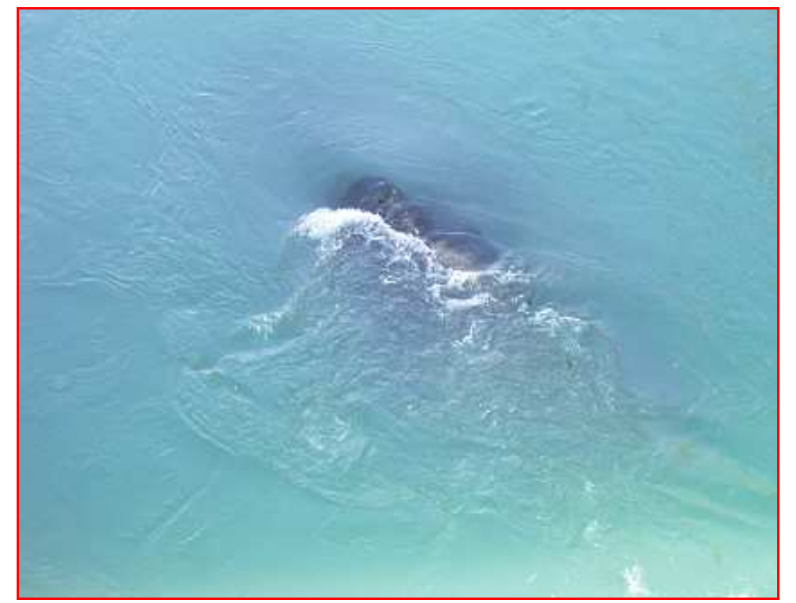

Figure 2: Turbulence as observed passing an obstacle in the same river of Figure 1, allows us to look at a clear example of wake.

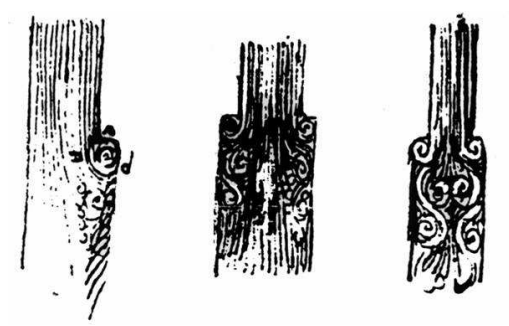

Figure 3: Three examples of vortices taken from the pictures by Leonardo da Vinci (cf. Frisch, 1995). 


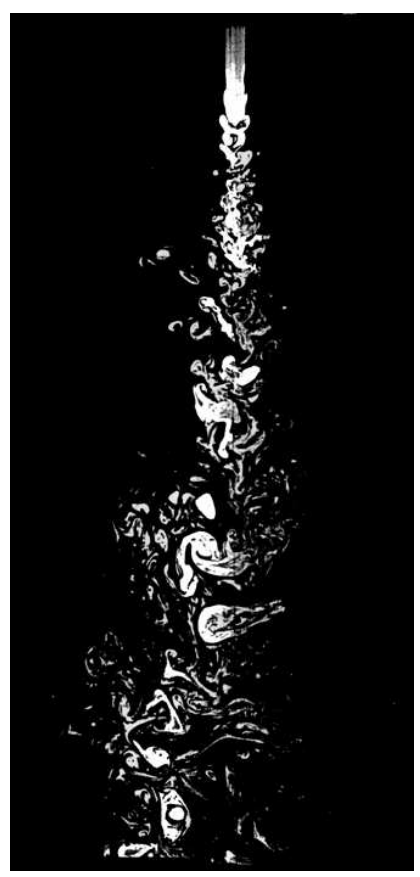

Figure 4: Turbulence as observed in a turbulent water jet (Van Dyke, 1982) reported in the book by Frisch (1995) (photograph by P. Dimotakis, R. Lye, and D. Papantoniu).

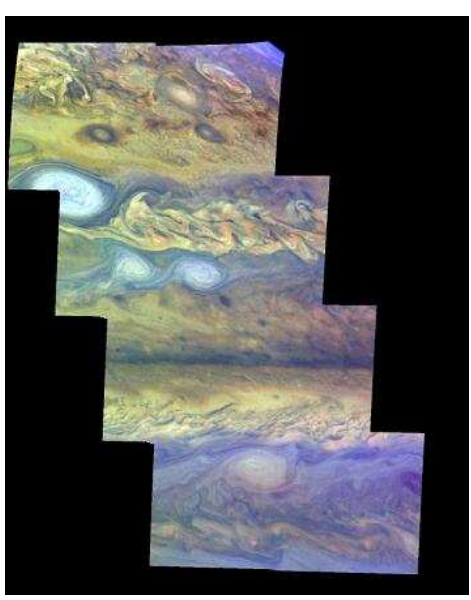

Figure 5: Turbulence in the atmosphere of Jupiter as observed by Voyager. 


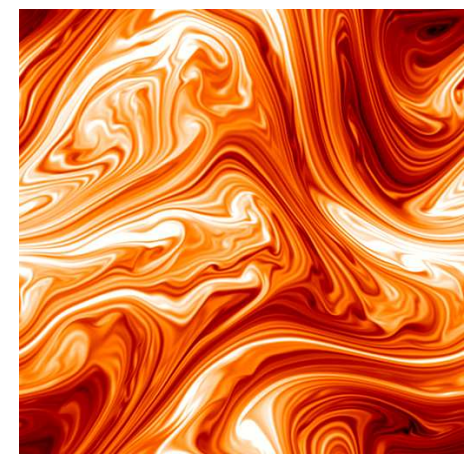

Figure 6: High resolution numerical simulations of 2D MHD turbulence at resolution $2048 \times 2048$ (courtesy by H. Politano). Here the authors show the current density $J(x, y)$, at a given time, on the plane $(x, y)$.

but details are not predictable. We have to restore a statistical approach to turbulence, just like random or stochastic processes, even if the problem is born within the strange dynamics of a deterministic system!

Turbulence increases the properties of transport in a flow. For example, the urban pollution, without atmospheric turbulence, would not be spread (or eliminated) in a relatively short time. Results from numerical simulations of the concentration of a passive scalar transported by a turbulent flow is shown in Figure 7. On the other hand, in laboratory plasmas inside devices designed to achieve thermo-nuclear controlled fusion, anomalous transport driven by turbulent fluctuations is the main cause for the destruction of magnetic confinement. Actually, we are far from the achievement of controlled thermo-nuclear fusion. Turbulence, then, acquires the strange feature of something to be avoided in some cases, or to be invoked in some other cases.

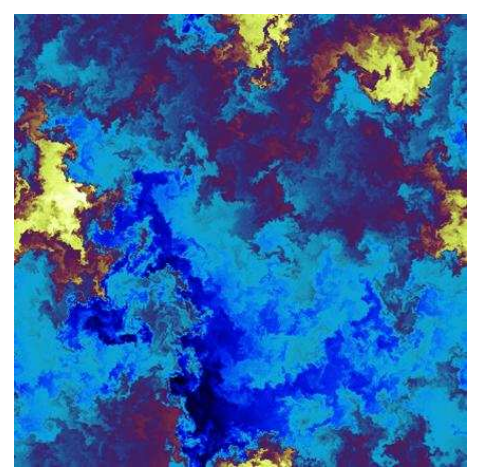

Figure 7: Concentration field $c(x, y)$, at a given time, on the plane $(x, y)$. The field has been obtained by a numerical simulation at resolution $2048 \times 2048$. The concentration is treated as a passive scalar, transported by a turbulent field. Low concentrations are reported in blue while high concentrations are reported in yellow (courtesy by A. Noullez).

Turbulence became an experimental science since O. Reynolds who, at the end of XIX century, observed and investigated experimentally the transition from laminar to turbulent flow. He noticed that the flow inside a pipe becomes turbulent every time a single parameter, a combination of the viscosity coefficient $\eta$, a characteristic velocity $U$, and length $L$, would increase. This parameter $R e=U L \rho / \eta$ ( $\rho$ is the mass density of the fluid) is now called the Reynolds number. At lower Re, 
say $R e \leq 2300$, the flow is regular (that is the motion is laminar), but when $R e$ increases beyond a certain threshold of the order of $R e \simeq 4000$, the flow becomes turbulent. As $R e$ increases, the transition from a laminar to a turbulent state occurs over a range of values of $R e$ with different characteristics and depending on the details of the experiment. In the limit $R e \rightarrow \infty$ the turbulence is said to be in a fully developed turbulent state. The original pictures by O. Reynolds is shown in Figure 8.

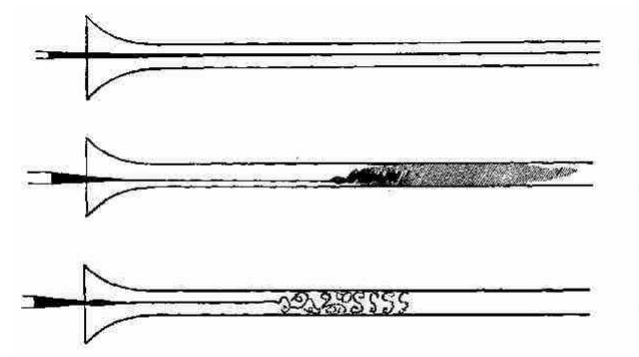

Figure 8: The original pictures by O. Reynolds which show the transition to a turbulent state of a flow in a pipe, as the Reynolds number increases from top to bottom (see the website Reynolds, 1883).

\subsection{Dynamics vs. statistics}

In Figure 9 a typical sample of turbulence as observed in a fluid flow in the Earth's atmosphere can be observed. Time evolution of both the longitudinal velocity component and the temperature is shown. Measurements in the solar wind show the same typical behavior. A typical sample of turbulence as measured by Helios 2 spacecraft is shown in Figure 10. A further sample of turbulence, namely the radial component of the magnetic field measured at the external wall of an experiment in a plasma device realized for thermonuclear fusion, is shown in Figure 11.

As it is well documented in these figures, the main feature of fully developed turbulence is the chaotic character of the time behavior. Said differently, this means that the behavior of the flow is unpredictable. While the details of fully developed turbulent motions are extremely sensitive to triggering disturbances, average properties are not. If this was not the case, there would be little significance in the averaging process. Predictability in turbulence can be recast at a statistical level. In other words, when we look at two different samples of turbulence, even collected within the same medium, we can see that details look very different. What is actually common is a generic stochastic behavior. This means that the global statistical behavior does not change going from one sample to the other. The idea that fully developed turbulent flows are extremely sensitive to small perturbations but have statistical properties that are insensitive to perturbations is of central importance throughout this review. Fluctuations of a certain stochastic variable $\psi$ are defined here as the difference from the average value $\delta \psi=\psi-\langle\psi\rangle$, where brackets means some averages. Actually, the method of taking averages in a turbulent flow requires some care. We would like to remind that there are, at least, three different kinds of averaging procedures that may be used to obtain statistically-averaged properties of turbulence. The space averaging is limited to flows that are statistically homogeneous or, at least, approximately homogeneous over scales larger than those of fluctuations. The ensemble averages are the most versatile, where average is taken over an ensemble of turbulent flows prepared under nearly identical external conditions. Of course, these flows are not nearly identical because of the large fluctuations present in turbulence. Each member of the ensemble is called a realization. The third kind of averaging procedure is the time average, which is useful only if the turbulence is statistically stationary over time scales much larger

Living Reviews in Solar Physics

http://www. livingreviews . org/lrsp-2005-4 


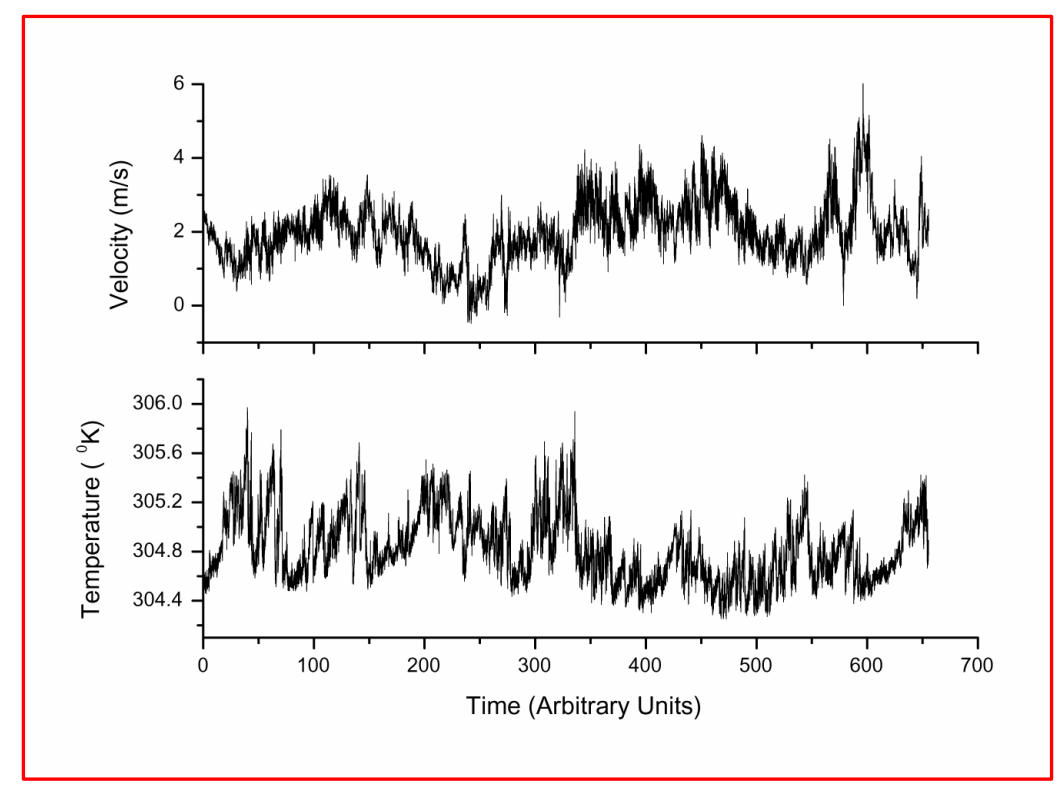

Figure 9: Turbulence as measured in the atmospheric boundary layer. Time evolution of the longitudinal velocity and temperature are shown in the upper and lower panels, respectively. The turbulent samples have been collected above a grass-covered forest clearing at $5 \mathrm{~m}$ above the ground surface and at a sampling rate of $56 \mathrm{~Hz}$ (Katul et al., 1997).

than the time scale of fluctuations. In practice, because of the convenience offered by locating a probe at a fixed point in space and integrating in time, experimental results are usually obtained as time averages. The ergodic theorem (Halmos, 1956) assures that time averages coincide with ensemble averages under some standard conditions (see Appendix 13).

A different property of turbulence is that all dynamically interesting scales are excited, that is, energy is spread over all scales. This can be seen in Figure 12 where we show the magnetic field intensity within a typical solar wind stream (see top panel). In the middle and bottom panels we show fluctuations at two different detailed scales. A kind of self-similarity (say a similarity at all scales) is observed.

Since fully developed turbulence involves a hierarchy of scales, a large number of interacting degrees of freedom are involved. Then, there should be an asymptotic statistical state of turbulence that is independent on the details of the flow. Hopefully, this asymptotic state depends, perhaps in a critical way, only on simple statistical properties like energy spectra, as much as in statistical mechanics equilibrium where the statistical state is determined by the energy spectrum (Huang, 1987). Of course, we cannot expect that the statistical state would determine the details of individual realizations, because realizations need not to be given the same weight in different ensembles with the same low-order statistical properties.

It should be emphasized that there are no firm mathematical arguments for the existence of an asymptotic statistical state. As we have just seen, reproducible statistical results are obtained from observations, that is, it is suggested experimentally and from physical plausibility. Apart from physical plausibility, it is embarrassing that such an important feature of fully developed turbulence, as the existence of a statistical stability, should remain unsolved. However, such is the complex nature of turbulence. 


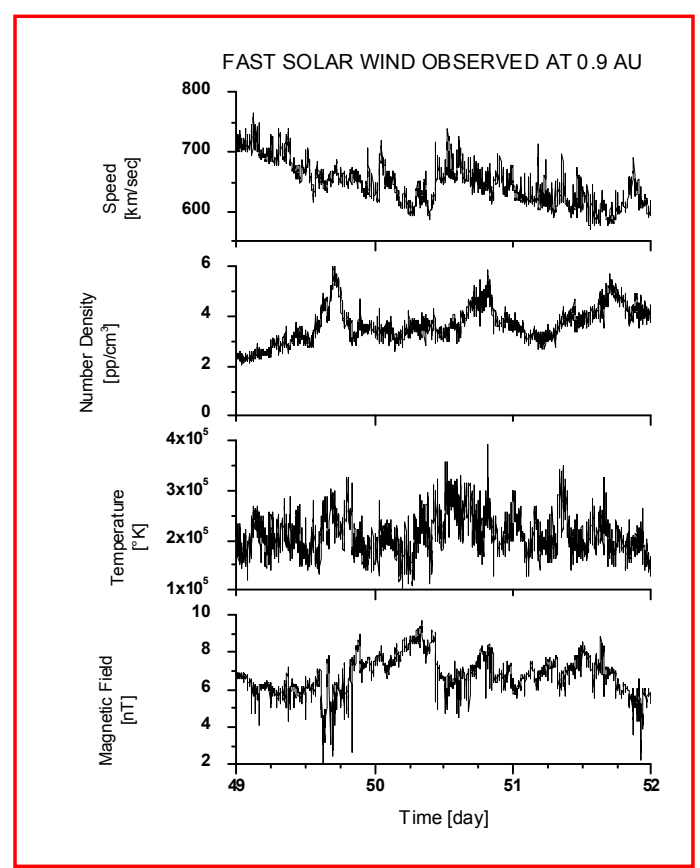

Figure 10: A sample of fast solar wind at distance $0.9 \mathrm{AU}$ measured by the Helios 2 spacecraft. From top to bottom: speed, number density, temperature, and magnetic field, as a function of time.

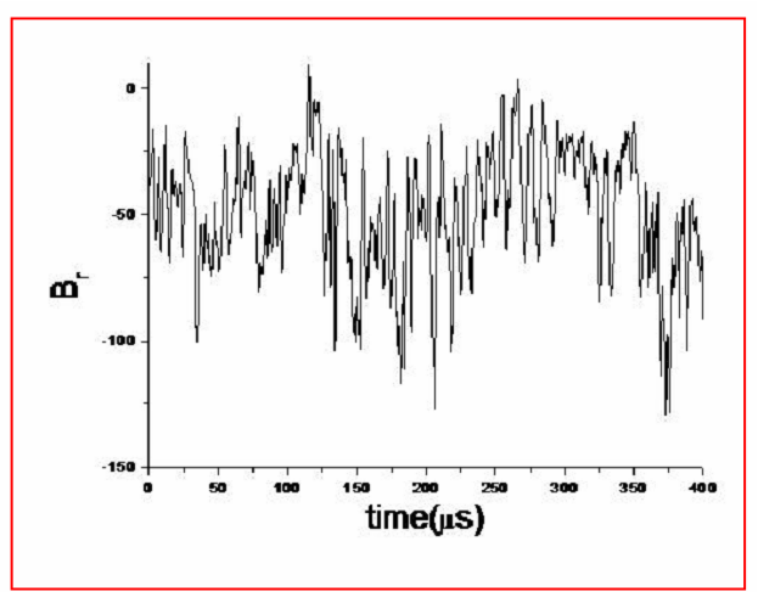

Figure 11: Turbulence as measured at the external wall of a device designed for thermonuclear fusion, namely the RFX in Padua (Italy). The radial component of the magnetic field as a function of time is shown in the figure (courtesy by V. Antoni).

Living Reviews in Solar Physics

http://www . livingreviews . org/lrsp-2005-4 


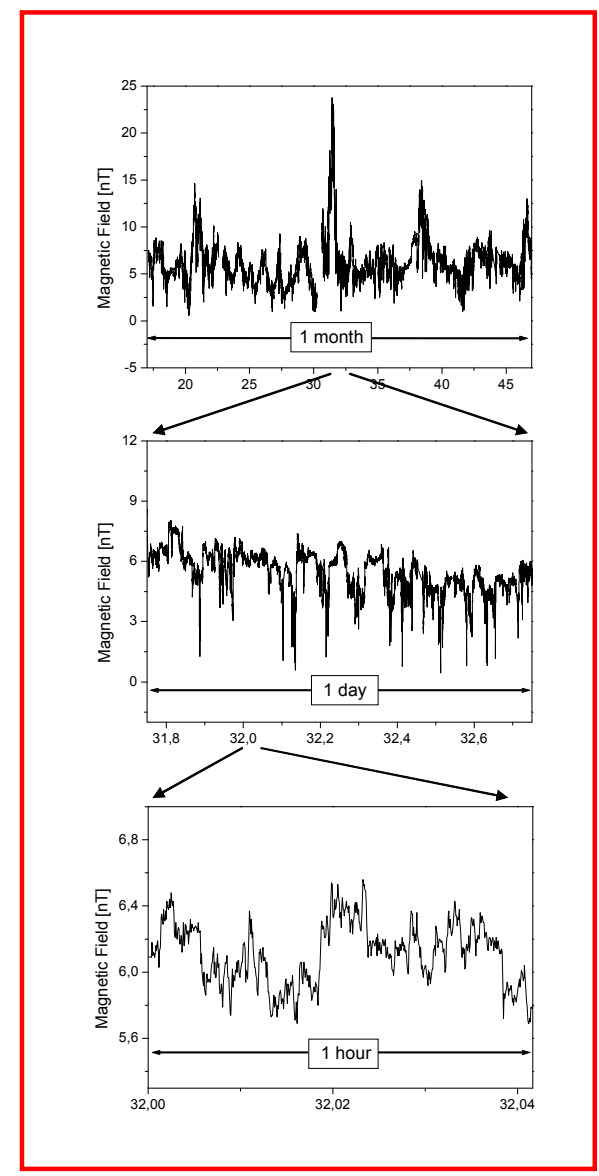

Figure 12: Magnetic intensity fluctuations as observed by Helios 2 in the inner solar wind at $0.9 \mathrm{AU}$, for different blow-ups. The apparent self-similarity is evident here. 


\section{Equations and Phenomenology}

In this section, we present the basic equations that are used to describe charged fluid flows, and the basic phenomenology of low-frequency turbulence. Readers interested in examining closely this subject can refer to the very wide literature on the subject of turbulence in fluid flows, as for example the recent books by, e.g., Pope (2000), McComb (1990), Frisch (1995) or many others, and the less known literature on MHD flows (Biskamp, 1993; Boyd and Sanderson, 2003; Biskamp, 2003). Plasma is seen as a continuous collisional medium so that all quantities are functions of space $\mathbf{r}$ and time $t$. Apart for the required quasi-neutrality, the basic assumption of MHD is that fields fluctuate on the same time and length scale as the plasma variables, say $\omega \tau_{\mathrm{H}} \simeq 1$ and $k L_{\mathrm{H}} \simeq 1$ ( $k$ and $\omega$ are, respectively, the wave number and the frequency of the fields, while $\tau_{\mathrm{H}}$ and $L_{\mathrm{H}}$ are the hydrodynamic time and length scale, respectively). Since the plasma is treated as a single fluid, we have to take the slow rates of ions. A simple analysis shows also that the electrostatic force and the displacement current can be neglected in the non-relativistic approximation. Then, MHD equations can be derived as shown in the following sections.

\subsection{The Navier-Stokes equation and the Reynolds number}

Equations which describe the dynamics of real incompressible fluid flows have been introduced by C. Navier in 1823 and improved by G. Stokes. They are nothing but the momentum equation based on Newton's second law, which relates the acceleration of a fluid particle ${ }^{2}$ to the resulting volume and body forces acting on it. These equations have been introduced by L. Euler, however, the main contribution by C. Navier was to add a friction forcing term due to the interactions between fluid layers which move with different speed. This term results to be proportional to the viscosity coefficients $\eta$ and $\xi$ and to the variation of speed. By defining the velocity field $\mathbf{u}(\mathbf{r}, t)$ the kinetic pressure $p$ and the density $\rho$, the equations describing a fluid flow are the continuity equation to describe the conservation of mass

$$
\frac{\partial \rho}{\partial t}+(\mathbf{u} \cdot \nabla) \rho=-\rho \nabla \cdot \mathbf{u}
$$

the equation for the conservation of momentum

$$
\rho\left[\frac{\partial \mathbf{u}}{\partial t}+(\mathbf{u} \cdot \nabla) \mathbf{u}\right]=-\nabla p+\eta \nabla^{2} \mathbf{u}+\left(\xi+\frac{\eta}{3}\right) \nabla(\nabla \cdot \mathbf{u}),
$$

and an equation for the conservation of energy

$$
\rho T\left[\frac{\partial s}{\partial t}+(\mathbf{u} \cdot \nabla) s\right]=\nabla \cdot(\chi \nabla T)+\frac{\eta}{2}\left(\frac{\partial u_{i}}{\partial x_{k}}+\frac{\partial u_{k}}{\partial x_{i}}-\frac{2}{3} \delta_{i k} \nabla \cdot \mathbf{u}\right)^{2}+\xi(\nabla \cdot \mathbf{u})^{2},
$$

where $s$ is the entropy per mass unit, $T$ is the temperature, and $\chi$ is the coefficient of thermoconduction. An equation of state closes the system of fluid equations.

The above equations considerably simplify if we consider the tractable incompressible fluid, where $\rho=$ const. so that we obtain the Navier-Stokes (NS) equation

$$
\frac{\partial \mathbf{u}}{\partial t}+(\mathbf{u} \cdot \nabla) \mathbf{u}=-\left(\frac{\nabla p}{\rho}\right)+\nu \nabla^{2} \mathbf{u}
$$

\footnotetext{
${ }^{2}$ A fluid particle is defined as an infinitesimal portion of fluid which moves with the local velocity. As usual in fluid dynamics, infinitesimal means small with respect to large scale, but large enough with respect to molecular scales.
}

Living Reviews in Solar Physics

http://www . livingreviews . org/Irsp-2005-4 
where the coefficient $\nu=\eta / \rho$ is the kinematic viscosity. The incompressibility of the flow translates in a condition on the velocity field, namely the field is divergence-free, i.e., $\nabla \cdot \mathbf{u}=0$. The nonlinear term in equations represents the convective (or substantial) derivative. Of course, we can add on the right hand side of this equation all external forces, which eventually act on the fluid parcel.

We use the velocity scale $U$ and the length scale $L$ to define dimensionless independent variables, namely $\mathbf{r}=\mathbf{r}^{\prime} L$ (from which $\nabla=\nabla^{\prime} / L$ ) and $t=t^{\prime}(L / U)$, and dependent variables $\mathbf{u}=\mathbf{u}^{\prime} U$ and $p=p^{\prime} U^{2} \rho$. Then, using these variables in Equation (4), we obtain

$$
\frac{\partial \mathbf{u}^{\prime}}{\partial t^{\prime}}+\left(\mathbf{u}^{\prime} \cdot \nabla^{\prime}\right) \mathbf{u}^{\prime}=-\nabla^{\prime} p^{\prime}+R e^{-1} \nabla^{\prime 2} \mathbf{u}^{\prime} .
$$

The Reynolds number $R e=U L / \nu$ is evidently the only parameter of the fluid flow. This defines a Reynolds number similarity for fluid flows, namely fluids with the same value of the Reynolds number behaves in the same way. Looking at Equation (5) it can be realized that the Reynolds number represents a measure of the relative strength between the non-linear convective term and the viscous term in Equation (4). The higher Re, the more important the non-linear term is in the dynamics of the flow. Turbulence is a genuine result of the non-linear dynamics of fluid flows.

\subsection{The coupling between a charged fluid and the magnetic field}

Magnetic fields are ubiquitous in the Universe and are dynamically important. At high frequencies, kinetic effects are dominant, but at frequencies lower than the ion cyclotron frequency, the evolution of plasma can be modeled using the MHD approximation. Furthermore, dissipative phenomena can be neglected at large scales although their effects will be felt because of non-locality of non-linear interactions. In the presence of a magnetic field, the Lorentz force $\mathbf{j} \times \mathbf{B}$, where $\mathbf{j}$ is the electric current density, must be added to the fluid equations, namely

$$
\rho\left[\frac{\partial \mathbf{u}}{\partial t}+(\mathbf{u} \cdot \nabla) \mathbf{u}\right]=-\nabla p+\eta \nabla^{2} \mathbf{u}+\left(\xi+\frac{\eta}{3}\right) \nabla(\nabla \cdot \mathbf{u})-\frac{1}{4 \pi} \mathbf{B} \times(\nabla \times \mathbf{B}),
$$

and the Joule heat must be added to the equation for energy

$$
\rho T\left[\frac{\partial s}{\partial t}+(\mathbf{u} \cdot \nabla) s\right]=\sigma_{i k} \frac{\partial u_{i}}{\partial x_{k}}+\chi \nabla^{2} T+\frac{c^{2}}{16 \pi^{2} \sigma}(\nabla \times \mathbf{B})^{2},
$$

where $\sigma$ is the conductivity of the medium, and we introduced the viscous stress tensor

$$
\sigma_{i k}=\eta\left(\frac{\partial u_{i}}{\partial x_{k}}+\frac{\partial u_{k}}{\partial x_{i}}-\frac{2}{3} \delta_{i k} \nabla \cdot \mathbf{u}\right)+\xi \delta_{i k} \nabla \cdot \mathbf{u} .
$$

An equation for the magnetic field stems from the Maxwell equations in which the displacement current is neglected under the assumption that the velocity of the fluid under consideration is much smaller than the speed of light. Then, using

$$
\nabla \times \mathbf{B}=\mu_{0} \mathbf{j}
$$

and the Ohm's law for a conductor in motion with a speed $\mathbf{u}$ in a magnetic field

$$
\mathbf{j}=\sigma(\mathbf{E}+\mathbf{u} \times \mathbf{B}),
$$

we obtain the induction equation which describes the time evolution of the magnetic field

$$
\frac{\partial \mathbf{B}}{\partial t}=\nabla \times(\mathbf{u} \times \mathbf{B})+\left(1 / \sigma \mu_{0}\right) \nabla^{2} \mathbf{B},
$$


together with the constraint $\nabla \cdot \mathbf{B}=0$ (no magnetic monopoles in the classical case).

In the incompressible case, where $\nabla \cdot \mathbf{u}=0$, MHD equations can be reduced to

$$
\frac{\partial \mathbf{u}}{\partial t}+(\mathbf{u} \cdot \nabla) \mathbf{u}=-\nabla P_{\text {tot }}+\nu \nabla^{2} \mathbf{u}+(\mathbf{b} \cdot \nabla) \mathbf{b}
$$

and

$$
\frac{\partial \mathbf{b}}{\partial t}+(\mathbf{u} \cdot \nabla) \mathbf{b}=-(\mathbf{b} \cdot \nabla) \mathbf{u}+\eta \nabla^{2} \mathbf{b}
$$

Here $P_{\text {tot }}$ is the total kinetic $P_{k}=n k T$ plus magnetic pressure $P_{\mathrm{m}}=B^{2} / 8 \pi$, divided by the constant mass density $\rho$. Moreover, we introduced the velocity variables $\mathbf{b}=\mathbf{B} / \sqrt{4 \pi \rho}$ and the magnetic diffusivity $\eta$.

Similar to the usual Reynolds number, a magnetic Reynolds number $R_{\mathrm{m}}$ can be defined, namely

$$
R_{\mathrm{m}}=\frac{\mathbf{c}_{\mathrm{A}} L_{0}}{\eta}
$$

where $\mathbf{c}_{\mathrm{A}}=\mathbf{B}_{\mathbf{0}} / \sqrt{4 \pi \rho}$ is the Alfvén speed related to the large-scale $L_{0}$ magnetic field $\mathbf{B}_{\mathbf{0}}$. This number in most circumstances in astrophysics is very large, but the ratio of the two Reynolds numbers or, in other words, the magnetic Prandtl number $P_{\mathrm{m}}=\nu / \eta$ can differ widely.

The change of variable due to Elsässer (1950), say $\mathbf{z}^{ \pm}=\mathbf{u} \pm \mathbf{b}^{\prime}$, where we explicitly use the background uniform magnetic field $\mathbf{b}^{\prime}=\mathbf{b}+\mathbf{c}_{\mathrm{A}}$ (at variance with the bulk velocity, the largest scale magnetic field cannot be eliminated through a Galilean transformation), leads to the more symmetrical form of the MHD equations in the incompressible case

$$
\frac{\partial \mathbf{z}^{ \pm}}{\partial t} \mp\left(\mathbf{c}_{\mathrm{A}} \cdot \nabla\right) \mathbf{z}^{ \pm}+\left(\mathbf{z}^{\mp} \cdot \nabla\right) \mathbf{z}^{ \pm}=-\nabla P_{\mathrm{tot}}{ }^{*}+\nu^{ \pm} \nabla^{2} \mathbf{z}^{ \pm}+\nu^{\mp} \nabla^{2} \mathbf{z}^{\mp}+\mathbf{F}^{ \pm}
$$

where $P_{\text {tot }}{ }^{*}$ is the total pressure, $2 \nu^{ \pm}=\nu \pm \eta$ are the dissipative coefficients, and $\mathbf{F}^{ \pm}$are eventual external forcing terms. The relations $\nabla \cdot \mathbf{z}^{ \pm}=\mathbf{0}$ complete the set of equations. On linearizing Equation (12) and neglecting both the viscous and the external forcing terms, we have

$$
\frac{\partial \mathbf{z}^{ \pm}}{\partial t} \mp\left(\mathbf{c}_{\mathrm{A}} \cdot \nabla\right) \mathbf{z}^{ \pm} \simeq 0
$$

which shows that $\mathbf{z}^{-}\left(\mathbf{x}-\mathbf{c}_{\mathrm{A}} t\right)$ describes Alfvénic fluctuations propagating in the direction of $\mathbf{B}_{\mathbf{0}}$, and $\mathbf{z}^{+}\left(\mathbf{x}+\mathbf{c}_{\mathrm{A}} t\right)$ describes Alfvénic fluctuations propagating opposite to $\mathbf{B}_{\mathbf{0}}$. Note that MHD Equations (12) have the same structure as the Navier-Stokes equation, the main difference stems from the fact that non-linear coupling happens only between fluctuations propagating in opposite directions. As we will see, this has a deep influence on turbulence described by MHD equations.

It is worthwhile to remark that in the classical hydrodynamics, dissipative processes are defined through three coefficients, namely two viscosities and one thermoconduction coefficient. In the hydromagnetic case the coefficients considerably increase. Apart from few additional electrical coeffcients, we have a large-scale (background) magnetic field $\mathbf{B}_{\mathbf{0}}$. This makes the MHD equations intrinsically anisotropic. Furthermore, the stress tensor (8) is deeply modified by the presence of a magnetic field $\mathbf{B}_{\mathbf{0}}$, in that kinetic viscous coefficients must depend on the magnitude and direction of the magnetic field (Braginskii, 1965). This has a strong influence on the determination of the Reynolds number.

\subsection{Scaling features of the equations}

The scaled Euler equations are the same as Equations $(4,5)$, but without the term proportional to $R^{-1}$. The scaled variables obtained from the Euler equations are, then, the same. Thus,

Living Reviews in Solar Physics

http: //www. livingreviews . org/lrsp-2005-4 
scaled variables exhibit scaling similarity, and the Euler equations are said to be invariant with respect to scale transformations. Said differently, this means that NS Equation (4) own scaling properties (Frisch, 1995), that is, there exists a class of solutions which are invariate under scaling transformations. Introducing a length scale $\ell$, it is straightforward to verify that the scaling transformations $\ell \rightarrow \lambda \ell^{\prime}$ and $\mathbf{u} \rightarrow \lambda^{h} \mathbf{u}^{\prime}$ ( $\lambda$ is a scaling factor and $h$ is a scaling index) leave invariate the inviscid NS equation for any scaling exponent $h$, providing $P \rightarrow \lambda^{2 h} P^{\prime}$. When the dissipative term is taken into account, a characteristic length scale exists, say the dissipative scale $\ell_{\mathrm{D}}$. From a phenomenological point of view, this is the length scale where dissipative effects start to be experienced by the flow. Of course, since $\nu$ is in general very low, we expect that $\ell_{D}$ is very small. Actually, there exists a simple relationship for the scaling of $\ell_{\mathrm{D}}$ with the Reynolds number, namely $\ell_{\mathrm{D}} \sim L R e^{-3 / 4}$. The larger the Reynolds number, the smaller the dissipative length scale.

As it is easily verified, ideal MHD equations display similar scaling features. Say the following scaling transformations $\mathbf{u} \rightarrow \lambda^{h} \mathbf{u}^{\prime}$ and $\mathbf{B} \rightarrow \lambda^{\beta} \mathbf{B}^{\prime}$ ( $\beta$ here is a new scaling index different from $h$ ), leave the inviscid MHD equations unchanged, providing $P \rightarrow \lambda^{2 \beta} P^{\prime}, T \rightarrow \lambda^{2 h} T^{\prime}$, and $\rho \rightarrow$ $\lambda^{2(\beta-h)} \rho^{\prime}$. This means that velocity and magnetic variables have different scalings, say $h \neq \beta$, only when the scaling for the density is taken into account. In the incompressible case, we cannot distinguish between scaling laws for velocity and magnetic variables.

\subsection{The non-linear energy cascade}

The basic properties of turbulence, as derived both from the Navier-Stokes equation and from phenomenological considerations, is the legacy of A.N. Kolmogorov ${ }^{3}$ (Frisch, 1995). Phenomenology is based on the old picture by Richardson who realized that turbulence is made by a collection of eddies at all scales. Energy, injected at a length scale $L$, is transferred by non-linear interactions to small scales where it is dissipated at a characteristic scale $\ell_{D}$, the length scale where dissipation takes place. The main idea is that at very large Reynolds numbers, the injection scale $L$ and the dissipative scale $\ell_{\mathrm{D}}$ are completely separated. In a stationary situation, the energy injection rate must be balanced by the energy dissipation rate and must also be the same as the energy transfer rate $\varepsilon$ measured at any scale $\ell$ within the inertial range $\ell_{\mathrm{D}} \ll \ell \ll L$.

Fully developed turbulence involves a hierarchical process, in which many scales of motion are involved. To look at this phenomenon it is often useful to investigate the behavior of the Fourier coefficients of the fields. Assuming periodic boundary conditions the $\alpha$-th component of velocity field can be Fourier decomposed as

$$
u_{\alpha}(\mathbf{r}, t)=\sum_{\mathbf{k}} u_{\alpha}(\mathbf{k}, t) \exp (i \mathbf{k} \cdot \mathbf{r}),
$$

where $\mathbf{k}=2 \pi \mathbf{n} / L$ and $\mathbf{n}$ is a vector of integers. When used in the Navier-Stokes equation, it is a simple matter to show that the non-linear term becomes the convolution sum

$$
\frac{\partial u_{\alpha}(\mathbf{k}, t)}{\partial t}=M_{\alpha \beta \gamma}(\mathbf{k}) \sum_{\mathbf{q}} u_{\gamma}(\mathbf{k}-\mathbf{q}, t) u_{\beta}(\mathbf{q}, t),
$$

where $M_{\alpha \beta \gamma}(\mathbf{k})=-i k_{\beta}\left(\delta_{\alpha \gamma}-k_{\alpha} k_{\beta} / k^{2}\right)$ (for the moment we disregard the linear dissipative term).

MHD equations can be written in the same way, say by introducing the Fourier decomposition for Elsässer variables

$$
z_{\alpha}^{ \pm}(\mathbf{r}, t)=\sum_{\mathbf{k}} z_{\alpha}^{ \pm}(\mathbf{k}, t) \exp (i \mathbf{k} \cdot \mathbf{r})
$$

and using this expression in the MHD equations we obtain an equation which describes the time evolution of each Fourier mode. However, the divergence-less condition means that not all Fourier

\footnotetext{
${ }^{3}$ The translation of the original paper by Kolmogorov (1941) can be found in the book by Hunt et al. (1991).
} 
modes are independent, rather $\mathbf{k} \cdot \mathbf{z}^{ \pm}(\mathbf{k}, t)=0$ means that we can project the Fourier coefficients on two directions which are mutually orthogonal and orthogonal to the direction of $\mathbf{k}$, that is,

$$
\mathbf{z}^{ \pm}(\mathbf{k}, t)=\sum_{a=1}^{2} z_{a}^{ \pm}(\mathbf{k}, t) \mathbf{e}^{(a)}(\mathbf{k})
$$

with the constraint that $\mathbf{k} \cdot \mathbf{e}^{(a)}(\mathbf{k})=0$. In presence of a background magnetic field we can use the well defined direction $\mathbf{B}_{\mathbf{0}}$, so that

$$
\mathbf{e}^{(1)}(\mathbf{k})=\frac{i \mathbf{k} \times \mathbf{B}_{\mathbf{0}}}{\left|\mathbf{k} \times \mathbf{B}_{\mathbf{0}}\right|} \quad ; \quad \mathbf{e}^{(2)}(\mathbf{k})=\frac{i \mathbf{k}}{|\mathbf{k}|} \times \mathbf{e}^{(1)}(\mathbf{k}) .
$$

Note that in the linear approximation where the Elsässer variables represent the usual MHD modes, $z_{1}^{ \pm}(\mathbf{k}, t)$ represent the amplitude of the Alfvén mode while $z_{2}^{ \pm}(\mathbf{k}, t)$ represent the amplitude of the incompressible limit of the magnetosonic mode. From MHD Equations (12) we obtain the following set of equations:

$$
\left[\frac{\partial}{\partial t} \mp i\left(\mathbf{k} \cdot \mathbf{c}_{\mathrm{A}}\right)\right] z_{a}^{ \pm}(\mathbf{k}, t)=\left(\frac{L}{2 \pi}\right)^{3} \sum_{\mathbf{p}+\mathbf{q}=\mathbf{k}}^{\delta} \sum_{b, c=1}^{2} A_{a b c}(-\mathbf{k}, \mathbf{p}, \mathbf{q}) z_{b}^{ \pm}(\mathbf{p}, t) z_{c}^{\mp}(\mathbf{q}, t) .
$$

The coupling coefficients, which satisfy the symmetry condition $A_{a b c}(\mathbf{k}, \mathbf{p}, \mathbf{q})=-A_{b a c}(\mathbf{p}, \mathbf{k}, \mathbf{q})$, are defined as

$$
A_{a b c}(-\mathbf{k}, \mathbf{p}, \mathbf{q})=\left[(i \mathbf{k})^{\star} \cdot \mathbf{e}^{(c)}(\mathbf{q})\right]\left[\mathbf{e}^{(a) *}(\mathbf{k}) \cdot \mathbf{e}^{(b)}(\mathbf{p})\right]
$$

and the sum in Equation (15) is defined as

$$
\sum_{\mathbf{p}+\mathbf{q}=\mathbf{k}}^{\delta} \equiv\left(\frac{2 \pi}{L}\right)^{3} \sum_{\mathbf{p}} \sum_{\mathbf{q}} \delta_{\mathbf{k}, \mathbf{p}+\mathbf{q}}
$$

where $\delta_{\mathbf{k}, \mathbf{p}+\mathbf{q}}$ is the Kronecher's symbol. Quadratic non-linearities of the original equations correspond to a convolution term involving wave vectors $\mathbf{k}, \mathbf{p}$ and $\mathbf{q}$ related by the triangular relation $\mathbf{p}=\mathbf{k}-\mathbf{q}$. Fourier coefficients locally couple to generate an energy transfer from any pair of modes $\mathbf{p}$ and $\mathbf{q}$ to a mode $\mathbf{k}=\mathbf{p}+\mathbf{q}$.

The pseudo-energies $E^{ \pm}(t)$ are defined as

$$
E^{ \pm}(t)=\frac{1}{2} \frac{1}{L^{3}} \int_{L^{3}}\left|\mathbf{z}^{ \pm}(\mathbf{r}, t)\right|^{2} d^{3} \mathbf{r}=\frac{1}{2} \sum_{\mathbf{k}} \sum_{a=1}^{2}\left|z_{a}^{ \pm}(\mathbf{k}, t)\right|^{2}
$$

and, after some algebra, it can be shown that the non-linear term of Equation (15) conserves separately $E^{ \pm}(t)$. This means that both the total energy $E(t)=E^{+}+E^{-}$and the cross-helicity $E_{\mathrm{C}}(t)=E^{+}-E^{-}$, say the correlation between velocity and magnetic field, are conserved in absence of dissipation and external forcing terms.

In the idealized homogeneous and isotropic situation we can define the pseudo-energy tensor, which using the incompressibility condition can be written as

$$
U_{a b}^{ \pm}(\mathbf{k}, t) \equiv\left(\frac{L}{2 \pi}\right)^{3}\left\langle z_{a}^{ \pm}(\mathbf{k}, t) z_{b}^{ \pm}(\mathbf{k}, t)\right\rangle=\left(\delta_{a b}-\frac{k_{a} k_{b}}{k^{2}}\right) q^{ \pm}(k),
$$

brackets being ensemble averages, where $q^{ \pm}(k)$ is an arbitrary odd function of the wave vector $k$ and represents the pseudo-energies spectral density. When integrated over all wave vectors under the assumption of isotropy

$$
\operatorname{Tr}\left[\int d^{3} \mathbf{k} U_{a b}^{ \pm}(\mathbf{k}, t)\right]=2 \int_{0}^{\infty} E^{ \pm}(k, t) d k,
$$

Living Reviews in Solar Physics

http: //www. livingreviews . org/lrsp-2005-4 
where we introduce the spectral pseudo-energy $E^{ \pm}(k, t)=4 \pi k^{2} q^{ \pm}(k, t)$. This last quantity can be measured, and it is shown that satisfies the equations

$$
\frac{\partial E^{ \pm}(k, t)}{\partial t}=T^{ \pm}(k, t)-2 \nu k^{2} E^{ \pm}(k, t)+F^{ \pm}(k, t) .
$$

We use $\nu=\eta$ in order not to worry about coupling between + and - modes in the dissipative range. Since the non-linear term conserves total pseudo-energies we have

$$
\int_{0}^{\infty} d k T^{ \pm}(k, t)=0
$$

so that, when integrated over all wave vectors, we obtain the energy balance equation for the total pseudo-energies

$$
\frac{d E^{ \pm}(t)}{d t}=\int_{0}^{\infty} d k F^{ \pm}(k, t)-2 \nu \int_{0}^{\infty} d k k^{2} E^{ \pm}(k, t) .
$$

This last equation simply means that the time variations of pseudo-energies are due to the difference between the injected power and the dissipated power, so that in a stationary state

$$
\int_{0}^{\infty} d k F^{ \pm}(k, t)=2 \nu \int_{0}^{\infty} d k k^{2} E^{ \pm}(k, t)=\epsilon^{ \pm} .
$$

Looking at Equation (16), we see that the role played by the non-linear term is that of a redistribution of energy among the various wave vectors. This is the physical meaning of the non-linear energy cascade of turbulence.

\subsection{The inhomogeneous case}

Equations (16) refer to the standard homogeneous and incompressible MHD. Of course, the solar wind is inhomogeneous and compressible and the energy transfer equations can be as complicated as we want by modeling all possible physical effects like, for example, the wind expansion or the inhomogeneous large-scale magnetic field. A set of equations for the cross-correlation functions of both Elsässer fluctuations have been developed independently by Marsch and Tu (1989), Zhou and Matthaeus (1990), Oughton and Matthaeus (1992), and Tu and Marsch (1990a), following Marsch and Mangeney (1987) (see review by Tu and Marsch, 1996), and are based on some rather strong assumptions: i) a two-scale separation, and ii) small-scale fluctuations are represented as a kind of stochastic process ( $\mathrm{Tu}$ and Marsch, 1996). These equations look quite complicated, and just a comparison based on order-of-magnitude estimates can be made between them and solar wind observations (Tu and Marsch, 1996).

\subsection{Dynamical system approach to turbulence}

In the limit of fully developed turbulence, when dissipation goes to zero, an infinite range of scales are excited, that is, energy lies over all available wave vectors. Dissipation takes place at a typical dissipation length scale which depends on the Reynolds number $R e$ through $\ell_{\mathrm{D}} \sim L R e^{-3 / 4}$ (for a Kolmogorov spectrum $\left.E(k) \sim k^{-5 / 3}\right)$. In 3D numerical simulations the minimum number of grid points necessary to obtain information on the fields at these scales is given by $N \sim\left(L / \ell_{\mathrm{D}}\right)^{3} \sim$ $R e^{9 / 4}$. This rough estimate shows that a considerable amount of memory is required when we want to perform numerical simulations with high $R e$. At the present, typical values of Reynolds numbers reached in 2D and 3D numerical simulations are of the order of $10^{4}$ and $10^{3}$, respectively. At these values the inertial range spans approximately one decade or little more. 
Given the situation described above, the question of the best description of dynamics which results from original equations, using only a small amount of degree of freedom, becomes a very important issue. This can be achieved by introducing turbulence models which are investigated using tools of dynamical system theory (Bohr et al., 1998). Dynamical models, then, represent minimal set of ordinary differential equations that can mimic the gross features of energy cascade in turbulence. These studies are motivated by the famous Lorenz's model (Lorenz, 1963) which, containing only three degrees of freedom, simulates the complex chaotic behavior of turbulent atmospheric flow, becoming a paradigm for the study of chaotic systems.

Up to the Lorenz's chaotic model, studies on the birth of turbulence dealt with linear and, very rarely, with weak non-linear evolution of external disturbances. The first physical model of laminar-turbulent transition is due to Landau and it is reported in the fourth volume of the course on Theoretical Physics (Landau and Lifshitz, 1971). According to this model, as the Reynolds number is increased, the transition is due to a serie of infinite Hopf bifurcations at fixed values of the Reynolds number. Each subsequent bifurcation adds a new incommensurate frequency to the flow which, in some sense, is obliged to become turbulent because the infinite number of degrees of freedom involved.

The Landau transition scenario is, however, untenable because few incommensurate frequencies cannot exist without coupling between them. Ruelle and Takens (1971) proposed a new mathematical model, according to which after few, usually three, Hopf bifurcations the flow becomes suddenly chaotic. In the phase space this state is characterized by a very intricate attracting subset, a strange attractor. The flow corresponding to this state is highly irregular and strongly dependent on initial conditions. This characteristic feature is now known as the butterfly effect and represents the true definition of deterministic chaos. These authors indicated as an example for the occurrence of a strange attractor the old strange time behavior of the Lorenz's model. The model is a paradigm for the occurrence of turbulence in a deterministic system, it reads

$$
\frac{d x}{d t}=P_{\mathrm{r}}(y-x), \quad \frac{d y}{d t}=R x-y-x z, \quad \frac{d z}{d t}=x y-b z,
$$

where $x(t), y(t)$, and $z(t)$ represent the first three modes of a Fourier expansion of fluid convective equations in the Boussinesq approximation, $P_{\mathrm{r}}$ is the Prandtl number, $b$ is a geometrical parameter, and $R$ is the ratio between the Raylaigh number and the critical Raylaigh number for convective motion. The time evolution of the variables $x(t), y(t)$, and $z(t)$ is reported in Figure 13. A reproduction of the Lorenz butterfly attractor, namely the projection of the variables on the plane $(x, z)$ is shown in Figure 14. A few years later, Gollub and Swinney (1975) performed very sophisticated experiments ${ }^{4}$, concluding that the transition to turbulence in a flow between co-rotating cylinders is described by the Ruelle and Takens (1971) model rather than by the Landau scenario.

After this discovery, the strange attractor model gained a lot of popularity, thus stimulating a great number of further studies on the time evolution of non-linear dynamical systems. An enormous number of papers on chaos rapidly appeared in literature, quite in all fields of physics, and transition to chaos became a new topic. Of course, further studies on chaos rapidly lost touch with turbulence studies and turbulence, as reported by Feynman et al. (1977), still remains ... the last great unsolved problem of the classical physics. Actually, since the solar wind is in a state of fully developed turbulence, the topic of the transition to turbulence is not so close to the main goal of this review. On the other hand, we like to cite recent theoretical efforts made by Chian and coworkers (Chian et al., 1998, 2003) related to the onset of Alfvénic turbulence. These authors, numerically solved the derivative non-linear Schrödinger equation (Mjolhus, 1976; Ghosh and Papadopoulos, 1987) which governs the spatio-temporal dynamics of non-linear Alfvén waves, and found that

\footnotetext{
${ }^{4}$ These authors were the first ones to use physical technologies and methodologies to investigate turbulent flows from an experimental point of view. Before them, experimental studies on turbulence were motivated mainly by engineering aspects.
}

Living Reviews in Solar Physics

http://www . livingreviews . org/Irsp-2005-4 


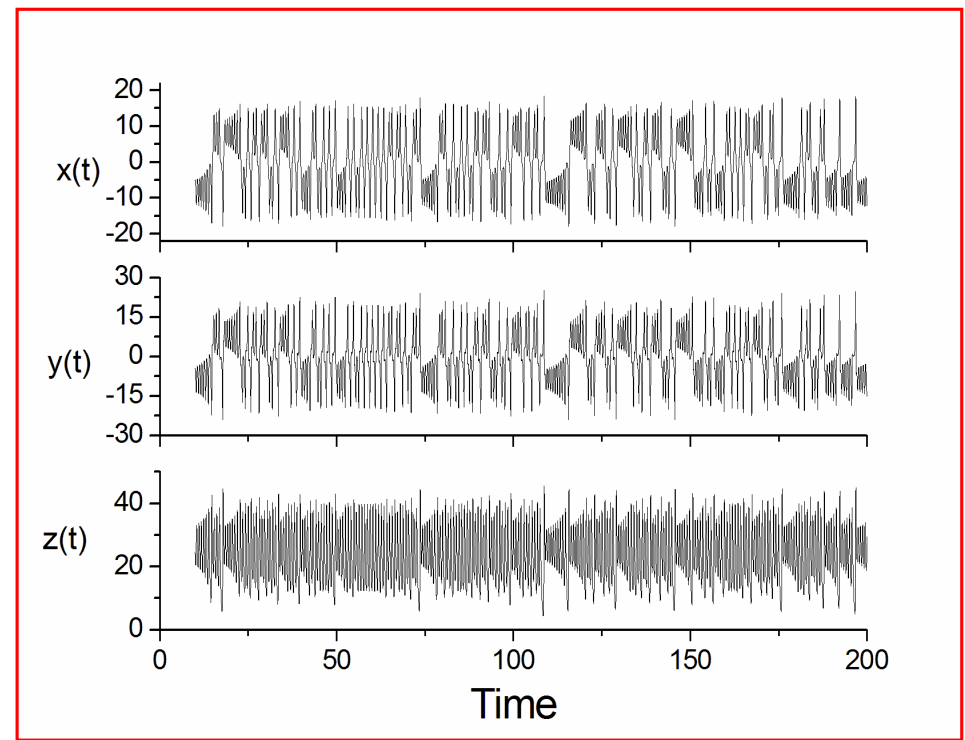

Figure 13: Time evolution of the variables $x(t), y(t)$, and $z(t)$ in the Lorenz's model (see Equation (18)). This figure has been obtained by using the parameters $P_{\mathrm{r}}=10, b=8 / 3$, and $R=28$.

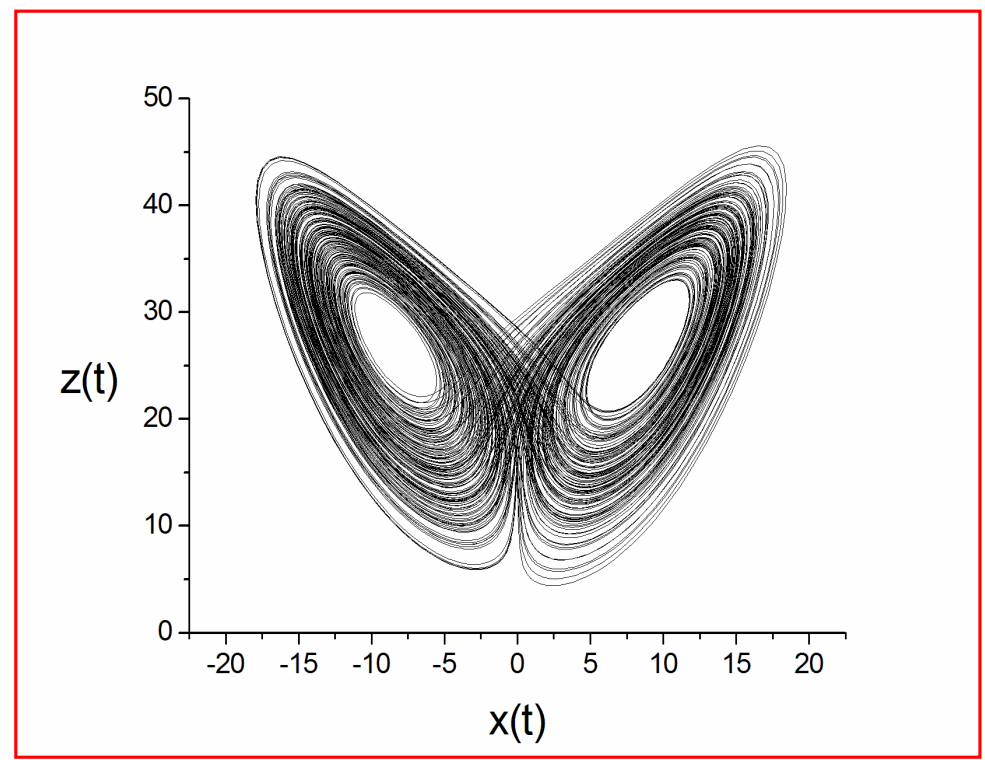

Figure 14: The Lorenz butterfly attractor, namely the time behavior of the variables $z(t)$ vs. $x(t)$ as obtained from the Lorenz's model (see Equation (18)). This figure has been obtained by using the parameters $P_{\mathrm{r}}=10, b=8 / 3$, and $R=28$. 
Alfvénic intermittent turbulence is characterized by strange attractors. Turbulence can evolve via two distinct routes: Pomeau-Manneville intermittency and crisis-induced intermittency. Both types of chaotic transitions follow episodic switching between different temporal behaviors. In one case (Pomeau-Manneville) the behavior of the magnetic fluctuations evolve from nearly periodic to chaotic while, in the other case the behavior intermittently assumes weakly chaotic or strongly chaotic features.

\subsection{Shell models for turbulence cascade}

Since numerical simulations, in some cases, cannot be used, simple dynamical systems can be introduced to investigate, for example, statistical properties of turbulent flows which can be compared with observations.

The shell model mimics the gross features of the time evolution of spectral Navier-Stokes or MHD equations. The 3D hydrodynamic shell model is usually quoted in literature as the GOY model, and has been introduced some time ago by Gledzer (1973) and by Ohkitani and Yamada (1989). The MHD shell model, which coincide with the GOY model when the magnetic variables are set to zero, has been introduced independently by Frick and Sokoloff (1998) and Giuliani and Carbone (1998). In the following, we will refer to the MHD shell model as the FSGC model. The shell model can be built up through four different steps:

\section{a) Introduce discrete wave vectors:}

As a first step we divide the wave vector space in a discrete number of shells whose radii grow according to a power $k_{n}=k_{0} \lambda^{n}$, where $\lambda>1$ is the inter-shell ratio, $k_{0}$ is the fundamental wave vector related to the largest available length scale $L$, and $n=1,2, \ldots, N$.

\section{b) Assign to each shell discrete scalar variables:}

Each shell is assigned two or more complex scalar variables $u_{n}(t)$ and $b_{n}(t)$, or Elsässer variables $Z_{n}^{ \pm}(t)=u_{n} \pm b_{n}(t)$. These variables describe the chaotic dynamics of modes in the shell of wave vectors between $k_{n}$ and $k_{n+1}$. It is worth noting that the discrete variable, mimicking the average behavior of Fourier modes within each shell, represents characteristic fluctuations across eddies at the scale $\ell_{n} \sim k_{n}^{-1}$. That is, the fields have the same scalings as field differences, for example $Z_{n}^{ \pm} \sim\left|Z^{ \pm}\left(x+\ell_{n}\right)-Z^{ \pm}(x)\right| \sim \ell_{n}^{h}$ in fully developed turbulence. In this way, the possibility to describe spatial behavior within the model is ruled out. We can only get, from a dynamical shell model, time series for shell variables at a given $k_{n}$, and we loose the fact that turbulence is a typical temporal and spatial complex phenomenon.

c) Introduce a dynamical model which describes non-linear evolution:

Looking at Equation (15) a model must have quadratic non-linearities among opposite variables $Z_{n}^{ \pm}(t)$ and $Z_{n}^{\mp}(t)$, and must couple different shells with free coupling coefficients.

\section{d) Fix as much as possible the coupling coefficients:}

This last step is not standard. A numerical investigation of the model might require the scanning of the properties of the system when all coefficients are varied. Coupling coefficients can be fixed by imposing the conservation laws of the original equations, namely the total pseudo-energies

$$
E^{ \pm}(t)=\frac{1}{2} \sum_{n}\left|Z_{n}^{ \pm}\right|^{2}
$$

that means the conservation of both the total energy and the cross-helicity:

$$
E(t)=\frac{1}{2} \sum_{n}\left|u_{n}\right|^{2}+\left|b_{n}\right|^{2} ; H_{c}(t)=\sum_{n} 2 \Re e\left(u_{n} b_{n}^{*}\right),
$$

Living Reviews in Solar Physics

http: //www. livingreviews . org/lrsp-2005-4 
where $\Re e$ indicates the real part of the product $u_{n} b_{n}^{*}$. As we said before, shell models cannot describe spatial geometry of non-linear interactions in turbulence, so that we loose the possibility of distinguishing between two-dimensional and three-dimensional turbulent behavior. The distinction is, however, of primary importance, for example as far as the dynamo effect is concerned in MHD. However, there is a third invariant which we can impose, namely

$$
H(t)=\sum_{n}(-1)^{n} \frac{\left|b_{n}\right|^{2}}{k_{n}^{\alpha}}
$$

which can be dimensionally identified as the magnetic helicity when $\alpha=1$, so that the shell model so obtained is able to mimic a kind of 3D MHD turbulence (Giuliani and Carbone, 1998).

After some algebra, taking into account both the dissipative and forcing terms, FSGC model can be written as

$$
\frac{d Z_{n}^{ \pm}}{d t}=i k_{n} \Phi_{n}^{ \pm *}+\frac{\nu \pm \mu}{2} k_{n}^{2} Z_{n}^{+}+\frac{\nu \mp \mu}{2} k_{n}^{2} Z_{n}^{-}+F_{n}^{ \pm},
$$

where

$$
\begin{aligned}
\Phi_{n}^{ \pm} & =\left(\frac{2-a-c}{2}\right) Z_{n+2}^{ \pm} Z_{n+1}^{\mp}+\left(\frac{a+c}{2}\right) Z_{n+1}^{ \pm} Z_{n+2}^{\mp}+ \\
& +\left(\frac{c-a}{2 \lambda}\right) Z_{n-1}^{ \pm} Z_{n+1}^{\mp}-\left(\frac{a+c}{2 \lambda}\right) Z_{n-1}^{\mp} Z_{n+1}^{ \pm}+ \\
& -\left(\frac{c-a}{2 \lambda^{2}}\right) Z_{n-2}^{\mp} Z_{n-1}^{ \pm}-\left(\frac{2-a-c}{2 \lambda^{2}}\right) Z_{n-1}^{\mp} Z_{n-2}^{ \pm},
\end{aligned}
$$

where ${ }^{5} \lambda=2, a=1 / 2$, and $c=1 / 3$. In the following, we will consider only the case where the dissipative coefficients are the same, i.e., $\nu=\mu$.

\subsection{The phenomenology of fully developed turbulence: Fluid-like case}

Here we present the phenomenology of fully developed turbulence, as far as the scaling properties are concerned. In this way we are able to recover a universal form for the spectral pseudo-energy in the stationary case. In real space a common tool to investigate statistical properties of turbulence is represented by field increments $\delta z_{\ell}^{ \pm}(\mathbf{r})=\left[\mathbf{z}^{ \pm}(\mathbf{r}+\ell)-\mathbf{z}^{ \pm}(\mathbf{r})\right] \cdot \mathbf{e}$, being e the longitudinal direction. These stochastic quantities represent fluctuations ${ }^{6}$ across eddies at the scale $\ell$. The scaling invariance of MHD equations (cf. Section 2.3), from a phenomenological point of view, implies that we expect solutions where $\delta z_{\ell}^{ \pm} \sim \ell^{h}$. All the statistical properties of the field depend only on the scale $\ell$, on the mean energy dissipation rate $\varepsilon^{ \pm}$, and on the viscosity $\nu$. Also, $\varepsilon^{ \pm}$is supposed to be the common value of the injection, transfer and dissipation rates. Moreover, the dependence on the viscosity only arises at small scales, near the bottom of the inertial range. Under these assumptions the typical energy dissipation rate per unit mass scales as $\varepsilon^{ \pm} \sim\left(\delta z_{\ell}^{ \pm}\right)^{2} / t_{\ell}^{ \pm}$. The time $t_{\ell}^{ \pm}$associated with the scale $\ell$ is the typical time needed for the energy to be transferred on a smaller scale, say the eddy turnover time $t_{\ell}^{ \pm} \sim \ell / \delta z_{\ell}^{\mp}$, so that

$$
\varepsilon^{ \pm} \sim\left(\delta z_{\ell}^{ \pm}\right)^{2} \delta z^{\mp} / \ell
$$

\footnotetext{
${ }^{5}$ We can use a different definition for the third invariant $H(t)$, for example a quantity positive defined, without the term $(-1)^{n}$ and with $\alpha=2$. This can be identified as the surrogate of the square of the vector potential, thus investigating a kind of $2 \mathrm{D}$ MHD. In this case, we obtain a shell model with $\lambda=2, a=5 / 4$, and $c=-1 / 3$. However, this model does not reproduce the inverse cascade of the square of magnetic potential observed in the true $2 \mathrm{D}$ MHD equations.

${ }^{6}$ We have already defined fluctuations of a field as the difference between the field itself and its average value. This quantity has been defined as $\delta \psi$. Here, the differences $\delta \psi_{\ell}$ of the field separated by a distance $\ell$ represents characteristic fluctuations at the scale $\ell$, say characteristic fluctuations of the field across specific structures (eddies) that are present at that scale. The reader can realize the difference between both definitions.
} 
When we conjecture that both $\delta z^{ \pm}$fluctuations have the same scaling laws, namely $\delta z^{ \pm} \sim \ell^{h}$, we recover the Kolmogorov scaling for the field increments

$$
\delta z_{\ell}^{ \pm} \sim\left(\varepsilon^{ \pm}\right)^{1 / 3} \ell^{1 / 3} .
$$

Usually, we refer to this scaling as the K41 model (Kolmogorov, 1941; Frisch, 1995). Note that, since from dimensional considerations the scaling of the energy transfer rate should be $\varepsilon^{ \pm} \sim \ell^{1-3 h}$, $h=1 / 3$ is the choice to guarantee the absence of scaling for $\varepsilon^{ \pm}$.

In the real space turbulence properties can be described using either the probability distribution functions (PDFs hereafter) of increments, or the longitudinal structure functions, which represents nothing but the higher order moments of the field. Disregarding the magnetic field, in a purely fully developed fluid turbulence, this is defined as $S_{\ell}^{(p)}=\left\langle\delta u_{\ell}^{p}\right\rangle$. These quantities, in the inertial range, behave as a power law $S_{\ell}^{(p)} \sim \ell^{\xi_{p}}$, so that it is interesting to compute the set of scaling exponent $\xi_{p}$. Using, from a phenomenological point of view, the scaling for field increments (see Equation (22)), it is straightforward to compute the scaling laws $S_{\ell}^{(p)} \sim \ell^{p / 3}$. Then $\xi_{p}=p / 3$ results to be a linear function of the order $p$.

When we assume the scaling law $\delta z_{\ell}^{ \pm} \sim \ell^{h}$, we can compute the high-order moments of the structure functions for increments of the Elsässer variables, namely $\left\langle\left(\delta z_{\ell}^{ \pm}\right)^{p}\right\rangle \sim \ell^{\xi_{p}}$, thus obtaining a linear scaling $\xi_{p}=p / 3$, similar to usual fluid flows. For Gaussianly distributed fields, a particular role is played by the second-order moment, because all moments can be computed from $S_{\ell}^{(2)}$. It is straightforward to translate the dimensional analysis results to Fourier spectra. The spectral property of the field can be recovered from $S_{\ell}^{(2)}$, say in the homogeneous and isotropic case

$$
S_{\ell}^{(2)}=4 \int_{0}^{\infty} E(k)\left(1-\frac{\sin k \ell}{k \ell}\right) d k
$$

where $k \sim 1 / \ell$ is the wave vector, so that in the inertial range where Equation (26) is verified

$$
E(k) \sim \varepsilon^{2 / 3} k^{-5 / 3} .
$$

The Kolmogorov spectrum (see Equation (23)) is largely observed in all experimental investigations of turbulence, and is considered as the main result of the K41 phenomenology of turbulence (Frisch, 1995). However, spectral analysis does not provide a complete description of the statistical properties of the field, unless this has Gaussian properties. The same considerations can be made for the spectral pseudo-energies $E^{ \pm}(k)$, which are related to the $2^{\text {nd }}$ order structure functions $\left\langle\left[\delta z_{\ell}^{ \pm}\right]^{2}\right\rangle$.

\subsection{The phenomenology of fully developed turbulence: Magnetically- dominated case}

The phenomenology of the magnetically-dominated case has been investigated by Iroshnikov (1963) and Kraichnan (1965), then developed by Dobrowolny et al. (1980b) to tentatively explain the occurrence of the observed Alfvénic turbulence, and finally by Carbone (1993) and Biskamp (1993) to get scaling laws for structure functions. It is based on the Alfvén effect, that is, the decorrelation of interacting eddies, which can be explained phenomenologically as follows. Since non-linear interactions happen only between opposite propagating fluctuations, they are slowed down (with respect to the fluid-like case) by the sweeping of the fluctuations across each other. This means that $\varepsilon^{ \pm} \sim\left(\delta z_{\ell}^{ \pm}\right)^{2} / T_{\ell}^{ \pm}$but the characteristic time $T_{\ell}^{ \pm}$required to efficiently transfer energy from an eddy to another eddy at smaller scales cannot be the eddy-turnover time, rather it is increased by a factor $t_{\ell}^{ \pm} / t_{\mathrm{A}}\left(t_{\mathrm{A}} \sim \ell / \mathbf{c}_{\mathrm{A}}<t_{\ell}^{ \pm}\right.$is the Alfvén time $)$, so that $T_{\ell}^{ \pm} \sim\left(t_{\ell}^{ \pm}\right)^{2} / t_{\mathrm{A}}$. Then, immediately

$$
\varepsilon^{ \pm} \sim \frac{\left[\delta z_{\ell}^{ \pm}\right]^{2}\left[\delta z_{\ell}^{\mp}\right]^{2}}{\ell \mathbf{c}_{\mathrm{A}}}
$$

Living Reviews in Solar Physics

http://www . livingreviews . org/lrsp-2005-4 
This means that both \pm modes are transferred at the same rate to small scales, namely $\epsilon^{+} \sim$ $\epsilon^{-} \sim \epsilon$, and this is the conclusion drawn by Dobrowolny et al. (1980b). In reality, this is not fully correct, namely the Alfvén effect yields to the fact that energy transfer rates have the same scaling laws for \pm modes but, we cannot say anything about the amplitudes of $\varepsilon^{+}$and $\varepsilon^{-}$(Carbone, 1993). Using the usual scaling law for fluctuations, it can be shown that the scaling behavior holds $\varepsilon \rightarrow \lambda^{1-4 h} \varepsilon^{\prime}$. Then, when the energy transfer rate is constant, we found a scaling law different from that of Kolmogorov and, in particular,

$$
\delta z_{\ell}^{ \pm} \sim\left(\varepsilon \mathbf{c}_{\mathrm{A}}\right)^{1 / 4} \ell^{1 / 4} .
$$

Using this phenomenology the high-order moments of fluctuations are given by $S_{\ell}^{(p)} \sim \ell^{p / 4}$. Even in this case, $\xi_{p}=p / 4$ results to be a linear function of the order $p$. The pseudo-energy spectrum can be easily found to be

$$
E(k) \sim\left(\varepsilon \mathbf{c}_{\mathrm{A}}\right)^{1 / 2} k^{-3 / 2} .
$$

This is the Iroshnikov-Kraichnan spectrum.

\subsection{Some exact relationships}

So far, we have been discussing about the inertial range of turbulence. What this means from a heuristic point of view is somewhat clear, but from an operational point of view this would be rather obscure. In this regard, a very important result on turbulence, due to Kolmogorov (1941), is the so called "4/5-law" which, being obtained from the Navier-Stokes equations, is "... one of the most important results in fully developed turbulence because it is both exact and nontrivial" (cf. Frisch, 1995). Under the hypothesis of homogeneity, isotropy, and, in the limit of infinite Reynolds number, assuming that the turbulent flow has a finite nonzero mean dissipation energy rate $\varepsilon$ (cf. Frisch, 1995), the third-order velocity structure function behaves linearly with $\ell$, namely

$$
S_{\ell}^{(3)}=-\frac{4}{5} \varepsilon \ell .
$$

Following a similar approach developed by Yaglom (1949), Politano and Pouquet (1995) derived an exact relation, from MHD equations, for the third-order correlator involving Elsässer variables:

$$
Y_{\ell}^{ \pm}=\left\langle\left[\delta z_{\ell}^{ \pm}\right]^{2} \delta z_{\ell}^{\mp}\right\rangle=-\frac{4}{3} \varepsilon^{ \pm} \ell
$$

Both Equations $(26,27)$ can be used, or better, in a certain sense they might be used, as a formal definition of inertial range. Since they are exact relationships derived from Navier-Stokes and MHD equations under usual hypotheses, they represent a kind of "zeroth-order" conditions on experimental and theoretical analysis of the inertial range properties of turbulence. Using Equation (27) for $Y_{\ell}^{ \pm}$, or better a little different form (Politano and Pouquet, 1995), namely $\left\langle\left[\delta z_{\ell}^{ \pm}\right]^{2}\left|\delta z_{\ell}^{\mp}\right|\right\rangle$, as a formal definition, these authors found that an inertial range is observed in numerical simulations (Politano et al., 1998a). At odds with numerical simulations, Equation (27) is not easily verified if applied to solar wind data since an extended range is not clearly defined. However, we will come back to this important point in the future version of this review, where we will show how $Y_{\ell}^{ \pm}$functions behave in the solar wind.

As far as the shell model is concerned, the existence of a cascade towards small scales is expressed by an exact relation, which is equivalent to Equation (27). Using Equations (20) the scale-by-scale pseudo-energy budget is given by

$$
\frac{d}{d t} \sum_{n}\left|Z_{n}^{ \pm}\right|^{2}=k_{n} \operatorname{Im}\left[T_{n}^{ \pm}\right]-\sum_{n} 2 \nu k_{n}^{2}\left|Z_{n}^{ \pm}\right|^{2}+\sum_{n} 2 \Re e\left[Z_{n}^{ \pm} F_{n}^{ \pm *}\right] .
$$


The second and third terms on the right hand side present, respectively, the rate of pseudo-energy dissipation and the rate of pseudo-energy injection. The first term represents the flux of pseudoenergy along the wave vectors, responsible for the redistribution of pseudo-energies on the wave vectors, and is given by

$$
\begin{aligned}
T_{n}^{ \pm} & =(a+c) Z_{n}^{ \pm} Z_{n+1}^{ \pm} Z_{n+2}^{\mp}+\left(\frac{2-a-c}{\lambda}\right) Z_{n-1}^{ \pm} Z_{n+1}^{ \pm} Z_{n}^{\mp}+ \\
& +(2-a-c) Z_{n}^{ \pm} Z_{n+2}^{ \pm} Z_{n+1}^{\mp}+\left(\frac{c-a}{\lambda}\right) Z_{)} Z_{n}^{ \pm} Z_{n+1}^{ \pm} Z_{n-1}^{\mp}
\end{aligned}
$$

Using the same assumptions as before, namely: i) the forcing terms act only on the largest scales, ii) the system can reach a statistically stationary state, and iii) in the limit of fully developed turbulence, $\nu \rightarrow 0$, the mean pseudo-energy dissipation rates tend to finite positive limits $\epsilon^{ \pm}$, it can be found that

$$
\left\langle T_{n}^{ \pm}\right\rangle=-\epsilon^{ \pm} k_{n}^{-1}
$$

This is an exact relation which is valid in the inertial range of turbulence. Even in this case it can be used as an operative definition of the inertial range in the shell model, that is, the inertial range of the energy cascade in the shell model is defined as the range of scales $k_{n}$, where the law from Equation (29) is verified. 


\section{Early Observations of MHD Turbulence in the Ecliptic}

Here we briefly present the history, since the first Mariner missions during the '60s, of the main steps towards the completion of an observational picture of turbulence in interplanetary space. This retrospective look at all the advances made in this field shows that space flights allowed us to discover a very large laboratory in space. As a matter of fact, in a wind tunnel we deal with characteristic dimensions of the order of $L \leq 10 \mathrm{~m}$ and probes of the size of about $d \simeq 1 \mathrm{~cm}$. In space, $L \simeq 10^{8} \mathrm{~m}$, while "probes" (say spacecrafts) are about $d \simeq 5 \mathrm{~m}$. We have a much larger laboratory but, up to now, we have made only single point measurements although the magnetospheric ESA-Cluster project has shown all the advantages of performing 3D measurements in space.

\subsection{Turbulence in the ecliptic}

When dealing with laboratory turbulence it is important to know all the aspects of the experimental device where turbulent processes take place in order to estimate related possible effects driven or influenced by the environment. In the solar wind, the situation is, in some aspects, similar although the plasma does not experience any confinement due to the "experimental device", which would be represented by free interplanetary space. However, it is a matter of fact that the turbulent state of the wind fluctuations and the subsequent radial evolution during the wind expansion greatly differ from fast to slow wind, and it is now well accepted that the macrostructure convected by the wind itself plays some role (see reviews by Tu and Marsch, 1995a; Goldstein et al., 1995b).

Fast solar wind originates from the polar regions of the Sun, within the open magnetic field line regions identified by coronal holes. Beautiful observations by SOHO spacecraft (see animation of Figure 15) have localized the birthplace of the solar wind within the intergranular lane, generally where three or more granules get together. Clear outflow velocities of up to $10 \mathrm{~km} \mathrm{~s}^{-1}$ have been recorded by SOHO/SUMER instrument (Hassler et al., 1999).

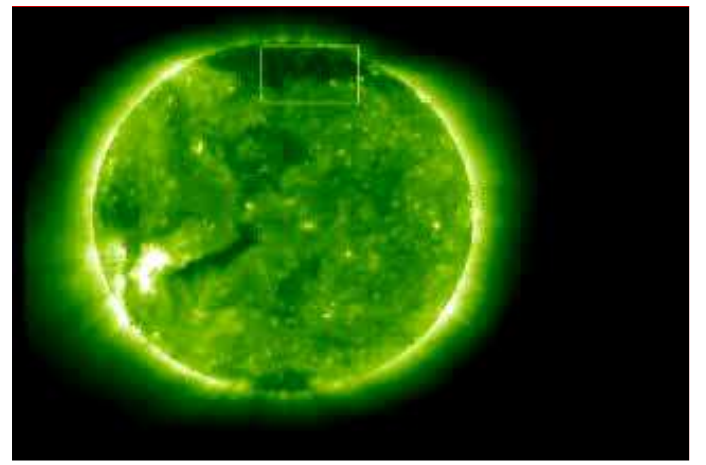

Figure 15: Still from a Animation built on SOHO/EIT and SOHO/SUMER observations of the solar-wind source regions and magnetic structure of the chromospheric network. Outflow velocities, at the network cell boundaries and lane junctions below the polar coronal hole, reach up to $10 \mathrm{~km} \mathrm{~s}^{-1}$ are represented by the blue colored areas (original figures from Hassler et al., 1999). (To watch the movie, please go to the online version of this review article at http://www . livingreviews.org/lrsp-2005-4.)

Slow wind, on the contrary, originates from the equatorial zone of the Sun. The slow wind plasma leaks from coronal features called "helmets", which can be easily seen protruding into the Sun's atmosphere during a solar eclipse (see Figure 16). Moreover, plasma emissions due to violent and abrupt phenomena also contribute to the solar wind in these regions of the Sun. 


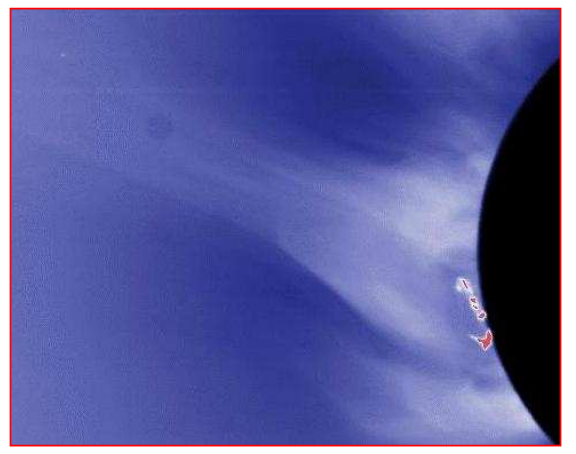

Figure 16: Helmet streamer during a solar eclipse. Slow wind leaks into the interplanetary space along the flanks of this coronal structure. (Figure taken from High Altitude Observatory, 1991).

However, this situation greatly changes during different phases of the solar activity cycle. Polar coronal holes, which during the maximum of activity are limited to small and not well defined regions around the poles, considerably widen up during solar minimum, reaching the equatorial regions (Forsyth et al., 1997; Forsyth and Breen, 2002; Balogh et al., 1999). This new configuration produces an alternation of fast and slow wind streams in the ecliptic plane, the plane where most of the spacecraft operate and record data. During the expansion, a dynamical interaction between fast and slow wind develops, generating the so called "stream interface", a thin region ahead of the fast stream characterized by strong compressive phenomena.

Figure 17 shows a typical situation in the ecliptic where fast streams and slow wind were observed by Helios $2 \mathrm{~s} / \mathrm{c}$ during its primary mission to the Sun. At that time, the spacecraft moved from $1 \mathrm{AU}$ (around day 17) to its closest approach to the Sun at 0.29 AU (around day 108). During this radial excursion, Helios 2 had a chance to observe the same corotating stream, that is plasma coming from the same solar source, at different heliocentric distances. This fortuitous circumstance, gave us the unique opportunity to study the radial evolution of turbulence under the reasonable hypothesis of time-stationarity of the source regions. Obviously, similar hypotheses decay during higher activity phase of the solar cycle since, as shown in Figure 18, the nice and regular alternation of fast corotating streams and slow wind is replaced by a much more irregular and spikey profile also characterized by a lower average speed.

Figure 19 focuses on a region centered on day 75, recognizable in Figure 17, when the s/c was at approximately $0.7 \mathrm{AU}$ from the Sun. Slow wind on the left hand side of the plot, fast wind on the right hand side, and the stream interface in between, can be clearly seen. This is a sort of canonical situation often encountered in the ecliptic, within the inner heliosphere, during solar activity minimum. Typical solar wind parameters, like proton number density $\rho_{p}$, proton temperature $T_{p}$, magnetic field intensity $|B|$, azimuthal angle $\Phi$, and elevation angle $\Theta$ are shown in the panels below the wind speed profile. A quick look at the data reveals that fast wind is less dense but hotter than slow wind. Moreover, both proton number density and magnetic field intensity are more steady and, in addition, the bottom two panels show that magnetic field vector fluctuates in direction much more than in slow wind. This last aspect unravels the presence of strong Alfvénic fluctuations which act mainly on magnetic field and velocity vector direction, and are typically found within fast wind (Belcher and Davis Jr, 1971; Belcher and Solodyna, 1975). The region just ahead of the fast wind, namely the stream interface, where dynamical interaction between fast and slow wind develops, is characterized by compressive effects which enhance proton density, temperature and field intensity. Within slow wind, a further compressive region precedes the stream interface but it is not due to dynamical effects but identifies the heliospheric current

Living Reviews in Solar Physics

http: //www . livingreviews . org/lrsp-2005-4 


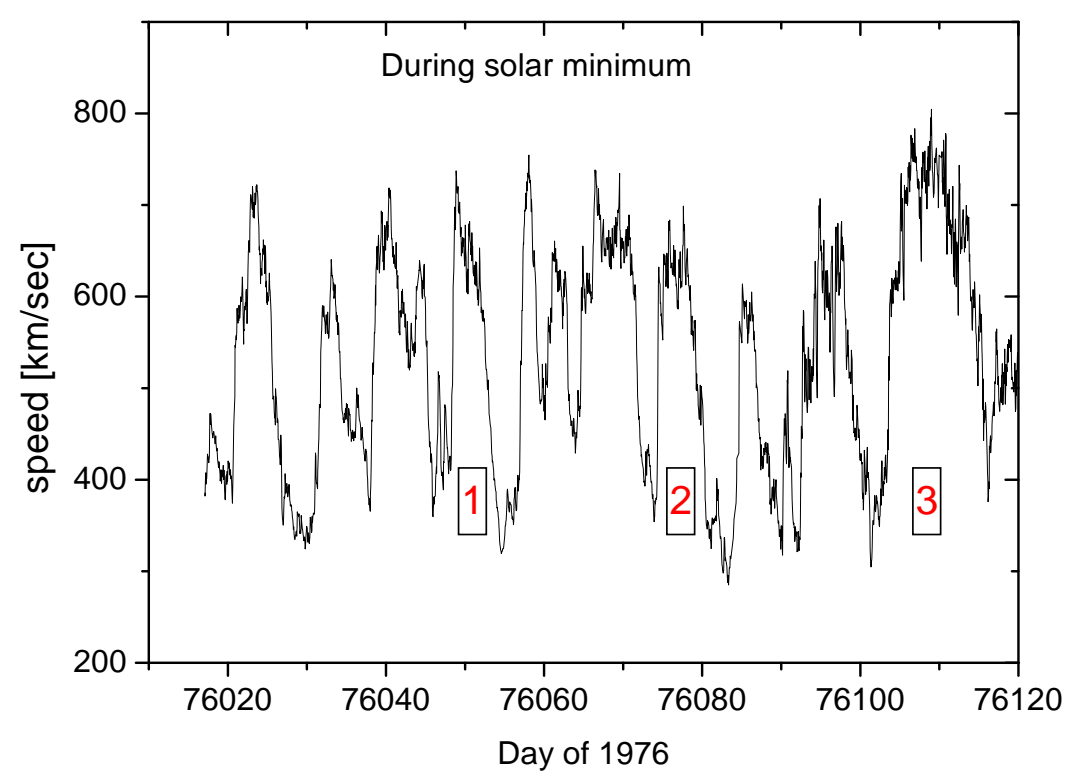

Figure 17: High velocity streams and slow wind as seen in the ecliptic during solar minimum as function of time [yyddd]. Streams identified by labels are the same corotating stream observed by Helios 2, during its primary mission to the sun in 1976, at different heliocentric distances. These streams, named "The Bavassano-Villante streams" after Tu and Marsch (1995a), have been of fundamental importance in understanding the radial evolution of MHD turbulence in the solar wind.

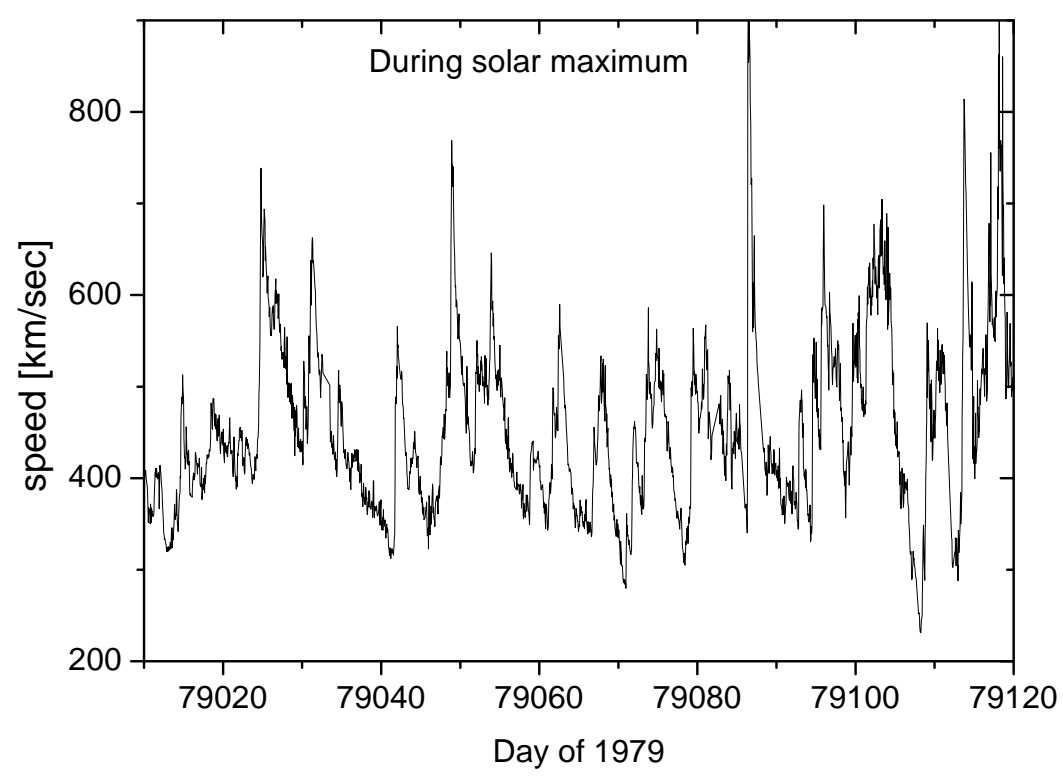

Figure 18: High velocity streams and slow wind as seen in the ecliptic during solar maximum. Data refer to Helios 2 observations in 1979. 


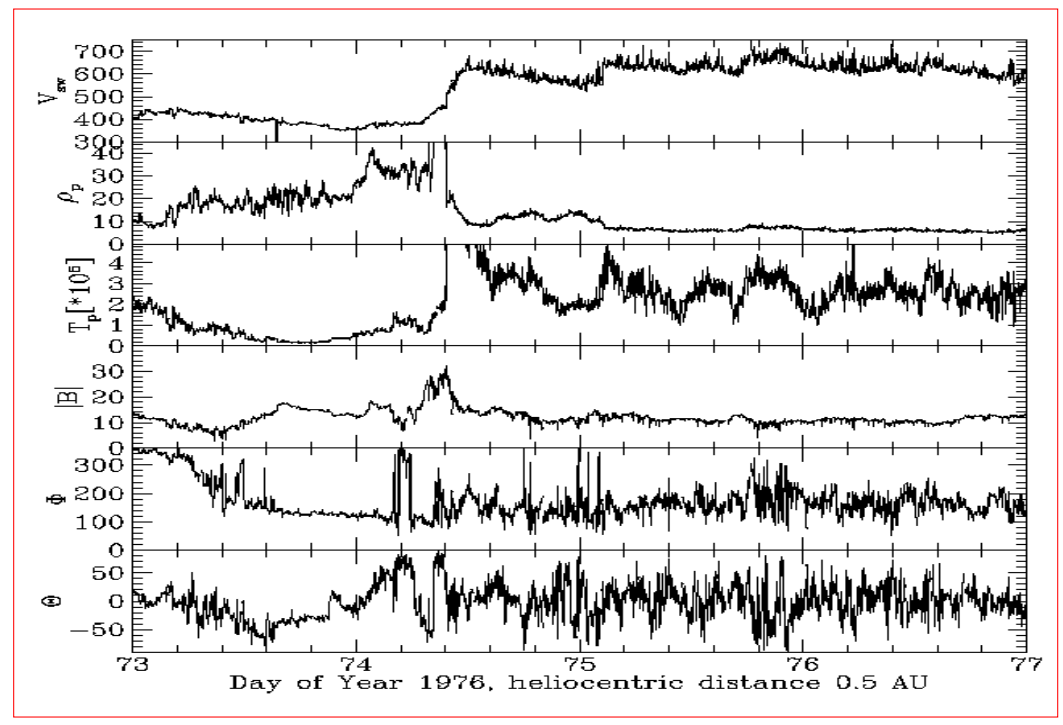

Figure 19: High velocity streams and slow wind as seen in the ecliptic during solar minimum

sheet, the surface dividing the two opposite polarities of the interplanetary magnetic field. As a matter of fact, the change of polarity can be noted within the first half of day 73 when the azimuthal angle $\Phi$ rotates by about $180^{\circ}$. Detailed studies (Bavassano et al., 1997) based on interplanetary scintillations (IPS) and in-situ measurements have been able to find a clear correspondence between the profile of path-integrated density obtained from IPS measurements and in-situ measurements by Helios 2 when the s/c was around 0.3 AU from the Sun.

Figure 20 shows measurements of several plasma and magnetic field parameters. The third panel from the top is the proton number density and it shows an enhancement within the slow wind just preceding the fast stream, as can be seen at the top panel. In this case the increase in density is not due to the dynamical interaction between slow and fast wind but it represents the profile of the heliospheric current sheet as sketched on the left panel of Figure 20. As a matter of fact, at these short distances from the Sun, dynamical interactions are still rather weak and this kind of compressive effects can be neglected with respect to the larger density values proper of the current sheet.

\subsubsection{Spectral properties}

First evidences of the presence of turbulent fluctuations were showed by Coleman (1968) who, using Mariner 2 magnetic and plasma observations, investigated the statistics of interplanetary fluctuations during the period August 27 - October 31, 1962, when the spacecraft orbited from 1.0 to 0.87 AU. At variance with Coleman (1968), Barnes and Hollweg (1974) analyzed the properties of the observed low-frequency fluctuations in terms of simple waves, disregarding the presence of an energy spectrum. Here we review the gross features of turbulence as observed in space by Mariner and Helios spacecrafts. By analyzing spectral densities, Coleman (1968) concluded that the solar wind flow is often turbulent, energy being distributed over an extraordinarily wide frequency range, from one cycle per solar rotation to $0.1 \mathrm{~Hz}$ !. The frequency spectrum, in a range of intermediate frequencies, was found to behave roughly as $f^{-1.2}$, the difference with the expected Kraichnan $f^{-1.5}$ spectral slope was tentatively attributed to the presence of high-frequency transverse fluctuations resulting from plasma garden-hose instability (Scarf et al., 1967). Waves generated by this

Living Reviews in Solar Physics

http: //www. livingreviews . org/lrsp-2005-4 


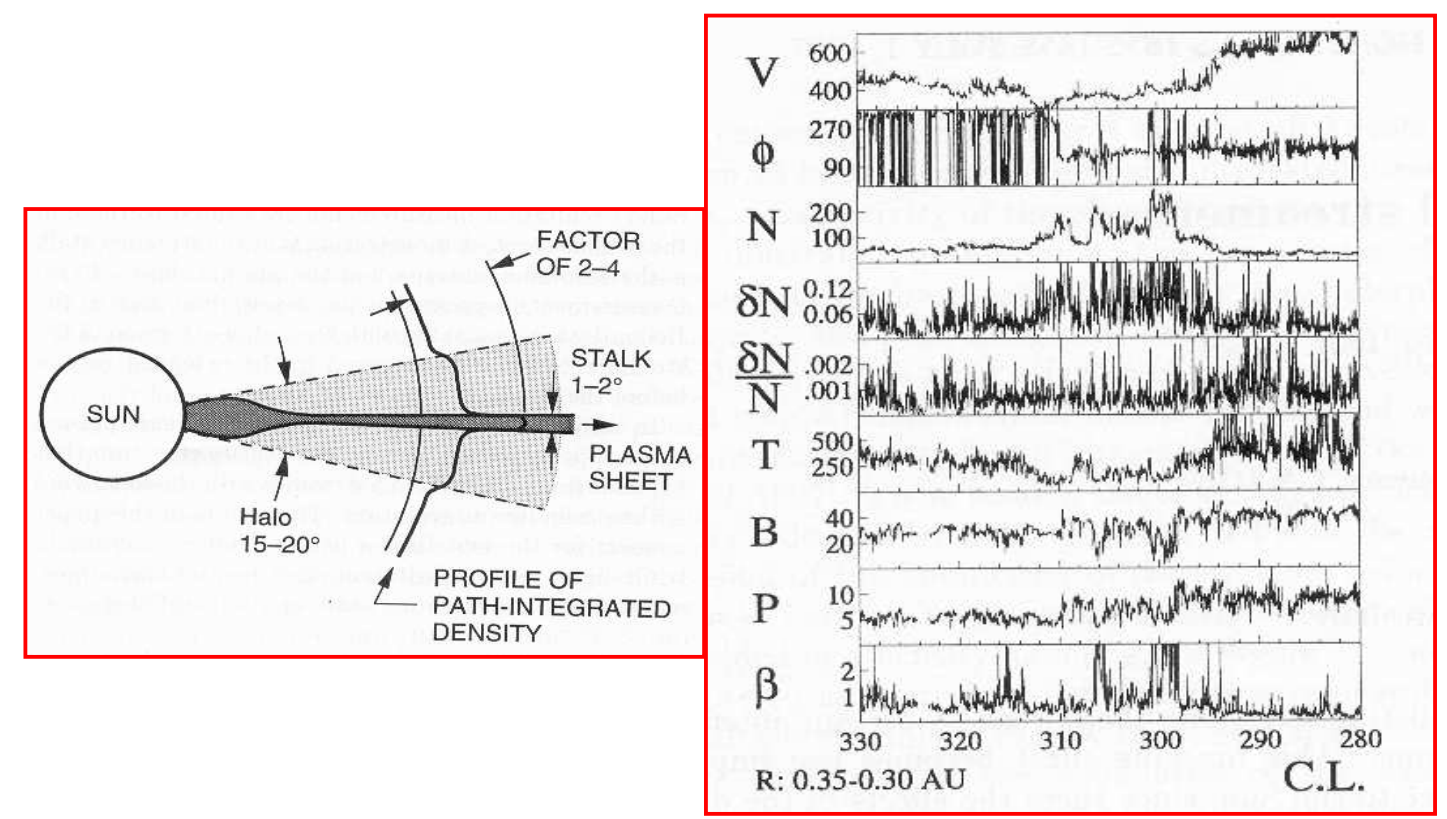

Figure 20: Left panel: a simple sketch showing the configuration of a helmet streamer and the density profile across this structure. Right panel: Helios 2 observations of magnetic field and plasma parameters across the heliospheric current sheet. From top to bottom: wind speed, magnetic field azimuthal angle, proton number density, density fluctuations and normalized density fluctuations, proton temperature, magnetic field magnitude, total pressure, and plasma beta, respectively (adopted from Bavassano et al., 1997, (c) 1997 American Geophysical Union, reproduced by permission of American Geophysical Union). 
instability contribute to the spectrum only in the range of frequencies near the proton cyclotron frequency, and would weaken the frequency dependence relatively to the Kraichnan scaling. The magnetic spectrum obtained by Coleman is shown in Figure 21.

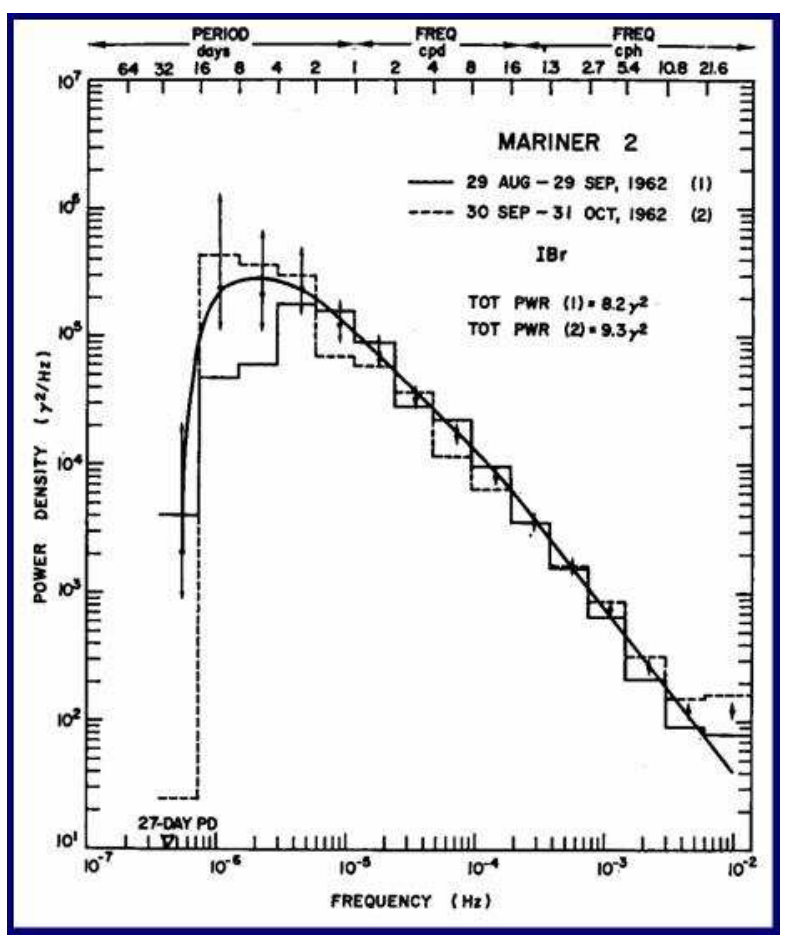

Figure 21: The magnetic energy spectrum as obtained by Coleman (1968).

Spectral properties of the interplanetary medium have been summarized by Russell (1972), who published a composite spectrum of the radial component of magnetic fluctuations as observed by Mariner 2, Mariner 4 and OGO 5 (see Figure 22). The frequency spectrum so obtained was divided into three main ranges: up to about $10^{-4} \mathrm{~Hz}$ the spectral slope was about $f^{-1}$; at intermediate frequencies $10^{-4} \leq f \leq 10^{-1} \mathrm{~Hz}$ a spectral slope of about $f^{-3 / 2}$ was found; finally, the high-frequency part of the spectrum, up to $1 \mathrm{~Hz}$, was characterized by a $f^{-2}$ dependence. The intermediate range ${ }^{7}$ of frequencies recalls spectral properties similar to those introduced by Kraichnan (1965) in the framework of MHD turbulence. It is worth reporting that scatter plots in the values of the spectral index of the intermediate region do not allow us to distinguish between a Kolmogorov spectrum $f^{-5 / 3}$ and a Kraichnan spectrum $f^{-3 / 2}$ (Veltri, 1980).

Then, as far as the solar wind turbulence concerns we do not think we should long discuss here whether or not solar wind developed turbulence be represented by $f^{-5 / 3}$ or $f^{-3 / 2}$, since observations showed that the slope is usually around $f^{-1.6}$ (Bavassano et al., 1982b; Tu and Marsch, 1995a) which, irony of fate, is just between the two cited values. Although we prefer to postpone to a future version of the present paper a detailed discussion on this topic and the related inertial range of solar wind fluctuations, it is worth citing that Tu et al. (1989c) already discussed this problem on the basis of Tu's model (Tu, 1988), using a variable ratio of the inward to outward

\footnotetext{
${ }^{7}$ To be precise, it is worth remarking that there are no convincing arguments to identify as inertial range the intermediate range of frequencies where the observed spectral properties are typical of fully developed turbulence. From a theoretical point of view, here the association "intermediate range" $\simeq$ "inertial range" is somewhat arbitrary as it can be inferred from the short discussion given in Section 2.10.
}

Living Reviews in Solar Physics

http://www . livingreviews . org/Irsp-2005-4 
Alfvénic energy as determined by observations of normalized cross-helicity. These values were then used to find the cascade constant that determines the level of the energy spectrum. The value they found for this constant resulted to be very close to the value observed in ordinary fluid turbulence, assuming that the correspondence between fluid and magnetofluid theories is reached by imposing zero cross-helicity for the MHD turbulence.

As a final comment, the situation of spectral indices determination in MHD turbulence is not changed since the '70s (cf. Carbone and Pouquet, 2005), numerical simulations deal with MHD flows of moderate Reynolds numbers and an inertial range is scarcely observed. The debate, after thirty years, is always open and contributions are welcome.

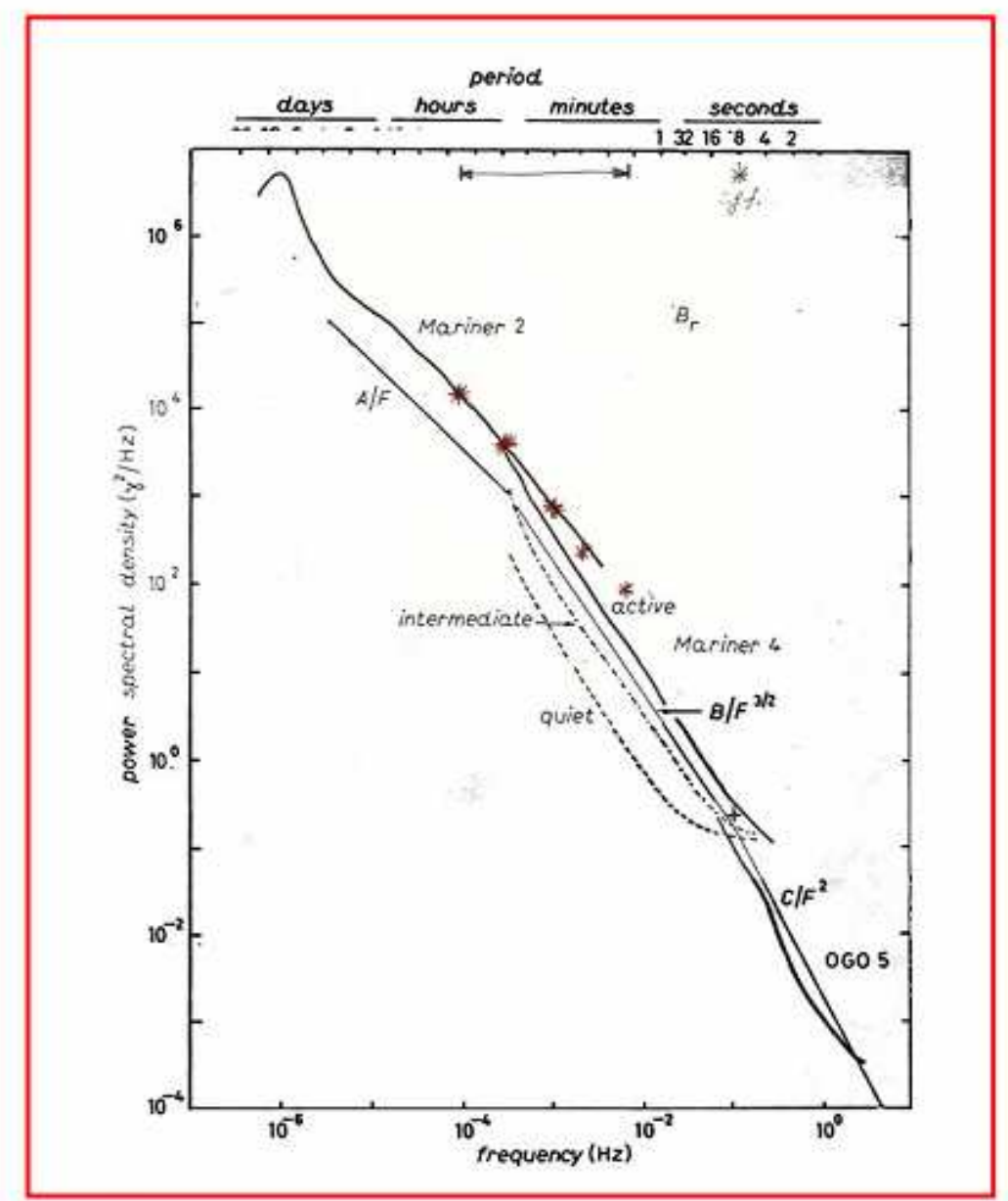

Figure 22: A composite figure of the magnetic spectrum obtained by Russell (1972).

\subsubsection{Evidence for non-linear interactions}

As we said previously, Helios $2 \mathrm{~s} / \mathrm{c}$ gave us the unique opportunity to study the radial evolution of turbulent fluctuations in the solar wind within the inner heliosphere. Most of the theoretical studies which aim to understand the physical mechanism at the base of this evolution originate 
from these observations (Bavassano et al., 1982b; Denskat and Neubauer, 1983). In Figure 23 we re-propose similar observations taken by Helios 2 during its primary mission to the Sun.

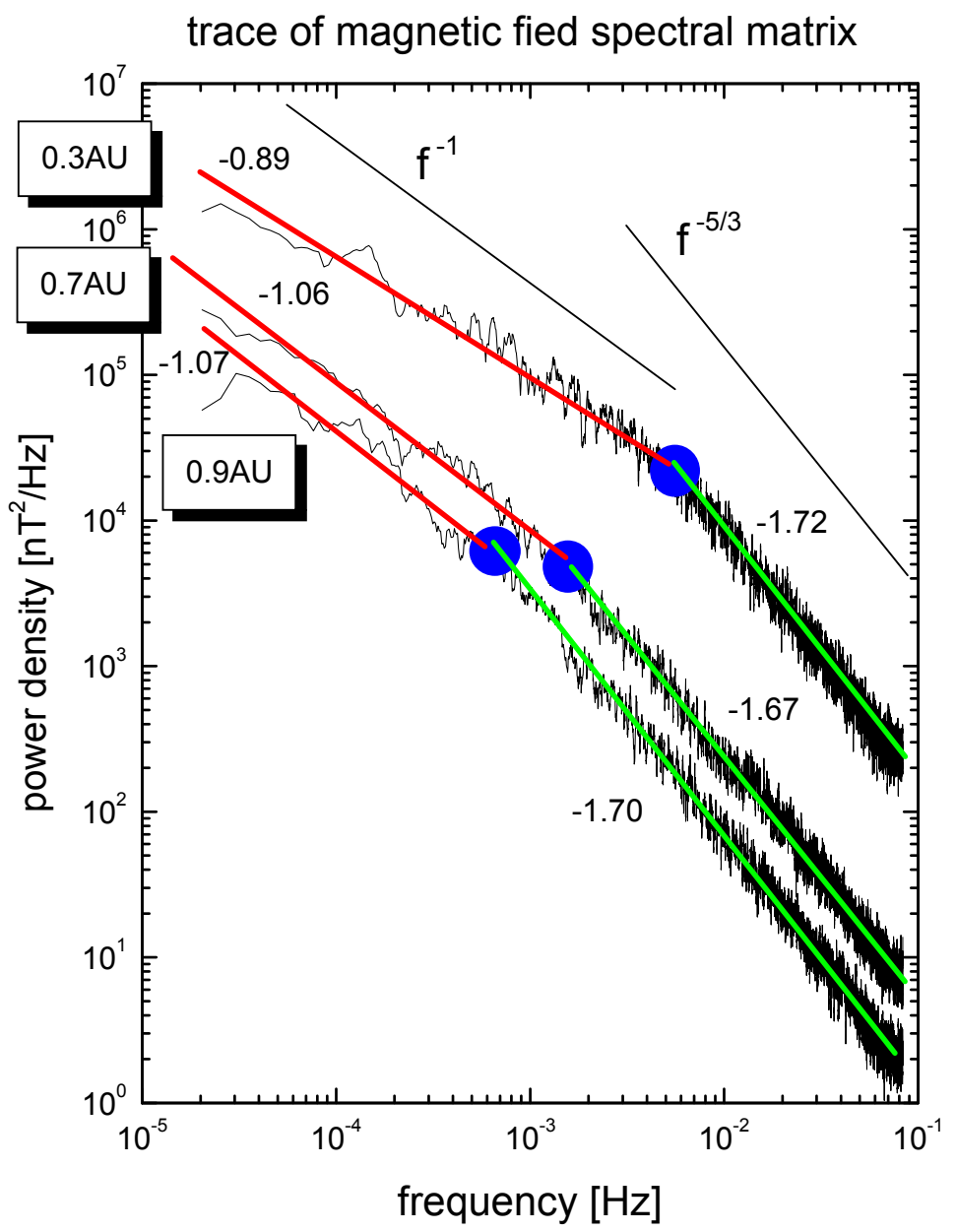

Figure 23: Power density spectra of magnetic field fluctuations observed by Helios 2 between 0.3 and $1 \mathrm{AU}$ within the trailing edge of the same corotating stream shown in Figure 17, during the first mission to the Sun in 1976. The spectral break (blue dot) shown by each spectrum, moves to lower and lower frequency as the heliocentric distance increases.

These power density spectra were obtained from the trace of the spectral matrix of magnetic field fluctuations, and belong to the same corotating stream observed by Helios 2 on day 49, at a heliocentric distance of $0.9 \mathrm{AU}$, on day 75 at $0.7 \mathrm{AU}$ and, finally, on day 104 at $0.3 \mathrm{AU}$. All the spectra are characterized by two distinct spectral slopes: about -1 within low frequencies and about a Kolmogorov like spectrum at higher frequencies. These two regimes are clearly separated by a knee in the spectrum often referred to as "frequency break". As the wind expands, the frequency break moves to lower and lower frequencies so that larger and larger scales become part of the Kolmogorov-like turbulence spectrum, i.e., of what we will indicate as "inertial range" (see discussion at the end of the previous section). Thus, the power spectrum of solar wind fluctuations is not solely function of frequency $f$, i.e., $P(f)$, but it also depends on heliocentric distance $r$, i.e.,

Living Reviews in Solar Physics

http: //www . livingreviews . org/lrsp-2005-4 
$P(f) \rightarrow P(f, r)$.

Matthaeus and Goldstein (1986) found the breakpoint around $10 \mathrm{~h}$ at $1 \mathrm{AU}$, and Klein et al. (1992) found that the breakpoint was near $16 \mathrm{~h}$ at $4 \mathrm{AU}$. This frequency break is strictly related to the correlation length (Klein, 1987) and the shift to lower frequency, during the wind expansion, is consistent with the growth of the correlation length observed in the inner (Bruno and Dobrowolny, 1986) and outer heliosphere (Matthaeus and Goldstein, 1982a). This phenomenology only apparently resembles hydrodynamic turbulence where the large eddies, below the frequency break, govern the whole process of energy cascade along the spectrum (Tu and Marsch, 1995b). As a matter of fact, when the relaxation time increases, the largest eddies provide the energy to be transferred along the spectrum and dissipated, with a decay rate approximately equal to the transfer rate and, finally, to the dissipation rate at the smallest wavelengths where viscosity dominates. Thus, we expect that the energy containing scales would loose energy during this process but would not become part of the turbulent cascade, say of the inertial range. Scales on both sides of the frequency break would remain separated. Accurate analysis performed in the solar wind (Bavassano et al., 1982b; Marsch and Tu, 1990b; Roberts, 1992) have shown that the low frequency range of the solar wind magnetic field spectrum radially evolves following the WKB model, or geometrical optics, which predicts a radial evolution of the power associated with the fluctuations $\sim r^{-3}$. Moreover, a steepening of the spectrum towards a Kolmogorov like spectral index can be observed. On the contrary, the same in-situ observations established that the radial decay for the higher frequencies was faster than $\sim r^{-3}$ and the overall spectral slope remained unchanged. This means that the energy contained in the largest eddies does not decay as it would happen in hydrodynamic turbulence and, as a consequence, the largest eddies cannot be considered equivalent to the energy containing eddies identified in hydrodynamic turbulence. So, this low frequency range is not separated from the inertial range but becomes part of it as the turbulence ages. These observations cast some doubts on the applicability of hydrodynamic turbulence paradigm to interplanetary MHD turbulence. A theoretical help came from adopting a local energy transfer function (Tu et al., 1984; Tu, 1987a,b, 1988), which would take into account the non-linear effects between eddies of slightly differing wave numbers, together with a WKB description which would mainly work for the large scale fluctuations. This model was able to reproduce most of the features observed in the magnetic power spectra $P(f, r)$ observed by Bavassano et al. (1982b). In particular, the concept of the "frequency break", just mentioned, was pointed out for the first time by Tu et al. (1984) who, developing the analytic solution for the radially evolving power spectrum $P(f, r)$ of fluctuations, obtained a critical frequency " $f_{\mathrm{c}}$ " such that for frequencies $f \ll f_{\mathrm{c}}, P(f, r) \propto f^{-1}$ and for $f \gg f_{\mathrm{c}}, P(f, r) \propto f^{-1.5}$. In addition, their model was the first model able to explain the decreasing of the "break frequency" with increasing heliocentric distance.

\subsubsection{Fluctuations anisotropy}

Interplanetary magnetic field (IMF) and velocity fluctuations are rather anisotropic as for the first time observed by Belcher and Davis Jr (1971), Belcher and Solodyna (1975), Chang and Nishida (1973), Burlaga and Turner (1976), Solodyna and Belcher (1976), Parker (1980), Bavassano et al. (1982a), Tu et al. (1989a), and Marsch and Tu (1990a). Moreover, this feature can be better observed if fluctuations are rotated into the minimum variance reference system (see Appendix 15).

Sonnerup and Cahill (1967) introduced the minimum variance analysis which consists of determining the eigenvectors of the matrix

$$
S_{i j}=\left\langle B_{i} B_{j}\right\rangle-\left\langle B_{i}\right\rangle\left\langle B_{j}\right\rangle,
$$

where $i$ and $j$ denote the components of magnetic field along the axes of a given reference system. The statistical properties of eigenvalues approximately satisfy the following statements: 
- One of the eigenvalues of the variance matrix is always much smaller than the others, say $\lambda_{1} \ll\left(\lambda_{2}, \lambda_{3}\right)$, and the corresponding eigenvector $\tilde{V}_{1}$ is the minimum-variance direction (see Appendix 15.1 for more details). This indicates that, at least locally, the magnetic fluctuations are confined in a plane perpendicular to the minimum-variance direction.

- In the plane perpendicular to $\tilde{V}_{1}$, fluctuations appear to be anisotropically distributed, say $\lambda_{3}>\lambda_{2}$. Typical values for eigenvalues are $\lambda_{3}: \lambda_{2}: \lambda_{1}=10: 3.5: 1.2$ (Chang and Nishida, 1973; Bavassano et al., 1982a).

- The direction $\tilde{V}_{1}$ is nearly parallel to the average magnetic field $\mathbf{B}_{\mathbf{0}}$, that is, the distribution of the angles between $\tilde{V}_{1}$ and $\mathbf{B}_{0}$ is narrow with width of about $10^{\circ}$ and centered around zero.

As shown in Figure 24, in this new reference system it is readily seen that the maximum and intermediate components have much more power compared with the minimum variance component. Generally, this kind of anisotropy characterizes Alfvénic intervals and, as such, it is more commonly found within high velocity streams (Marsch and Tu, 1990a).

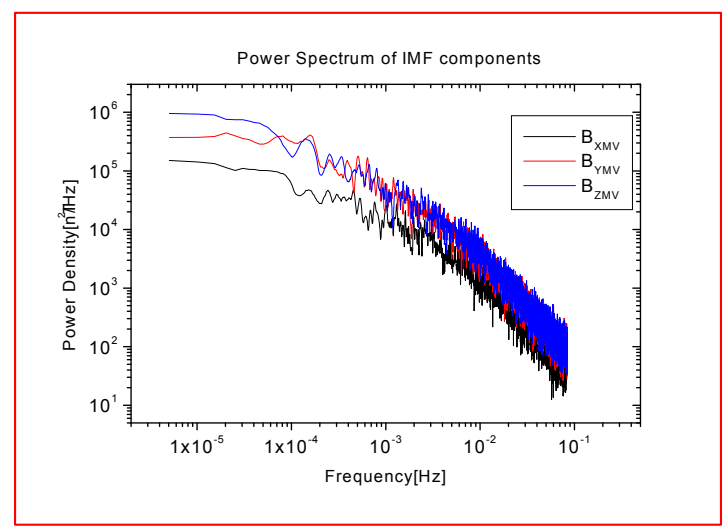

Figure 24: Power density spectra of the three components of IMF after rotation into the minimum variance reference system. The black curve corresponds to the minimum variance component, the blue curve to the maximum variance, and the red one to the intermediate component. This case refers to fast wind observed at $0.3 \mathrm{AU}$ and the minimum variance direction forms an angle of $\sim 8^{\circ}$ with respect to the ambient magnetic field direction. Thus, most of the power is associated with the two components quasi-transverse to the ambient field

A systematic analysis for both magnetic and velocity fluctuations was performed by Klein et al. $(1991,1993)$ between 0.3 and 10 AU. These studies showed that magnetic field and velocity minimum variance directions are close to each other within fast wind and mainly clustered around the local magnetic field direction. The effects of expansion are such to separate field and velocity minimum variance directions. While magnetic field fluctuations keep their minimum variance direction loosely aligned with the mean field direction, velocity fluctuations tend to have their minimum variance direction oriented along the radial direction. The depleted alignment to the background magnetic field would suggest a smaller anisotropy of the fluctuations. As a matter of fact, Klein et al. (1991) found that the degree of anisotropy, which can be defined as the ratio between the power perpendicular to and that along the minimum variance direction, decreases with heliocentric distance in the outer heliosphere.

At odds with these conclusions were the results by Bavassano et al. (1982a) who showed that the ratio $\lambda_{1} / \lambda_{3}$, calculated in the inner heliosphere within a corotating high velocity stream, clearly

Living Reviews in Solar Physics

http://www . livingreviews . org/lrsp-2005-4 
decreased with distance, indicating that the degree of magnetic anisotropy increased with distance. Moreover, this radial evolution was more remarkable for fluctuations of the order of a few hours than for those around a few minutes. Results by Klein et al. (1991) in the outer heliosphere and by Bavassano et al. (1982a) in the inner heliosphere remained rather controversial until recent studies (see Section 9.1), performed by Bruno et al. (1999b), found a reason for this discrepancy.

\subsubsection{Simulations of anisotropic MHD}

In the presence of a DC background magnetic field $\mathbf{B}_{\mathbf{0}}$ which, differently from the bulk velocity field, cannot be eliminated by a Galilean transformation, MHD incompressible turbulence becomes anisotropic (Shebalin et al., 1983; Carbone and Veltri, 1990). The main effect produced by the presence of the background field is to generate an anisotropic distribution of wave vectors as a consequence of the dependence of the characteristic time for the non-linear coupling on the angle between the wave vector and the background field. This effect can be easily understood if one considers the MHD equation. Due to the presence of a term $\left(\mathbf{B}_{\mathbf{0}} \cdot \nabla\right) \mathbf{z}^{ \pm}$, which describes the convection of perturbations in the average magnetic field, the non-linear interactions between Alfvénic fluctuations are weakened, since convection decorrelates the interacting eddies on a time of the order $\left(\mathbf{k} \cdot \mathbf{B}_{\mathbf{0}}\right)^{-1}$. Clearly fluctuations with wave vectors almost perpendicular to $\mathbf{B}_{\mathbf{0}}$ are interested by such an effect much less than fluctuations with $\mathbf{k} \| \mathbf{B}_{\mathbf{0}}$. As a consequence, the former are transferred along the spectrum much faster than the latter (Shebalin et al., 1983; Grappin, 1986; Carbone and Veltri, 1990).

To quantify anisotropy in the distribution of wave vectors $\mathbf{k}$ for a given dynamical variable $Q(\mathbf{k}, t)$ (namely the energy, cross-helicity, etc.), it is useful to introduce the parameter

$$
\Omega_{Q}=\tan ^{-1} \sqrt{\frac{\left\langle k_{\perp}^{2}\right\rangle_{Q}}{2\left\langle k_{\|}^{2}\right\rangle_{Q}}}
$$

(Shebalin et al., 1983; Carbone and Veltri, 1990), where the average of a given quantity $g(\mathbf{k})$ is defined as

$$
\langle g(\mathbf{k})\rangle_{Q}=\frac{\int d^{3} \mathbf{k} g(\mathbf{k}) Q(\mathbf{k}, t)}{\int d^{3} \mathbf{k} Q(\mathbf{k}, t)} .
$$

For a spectrum with wave vectors perpendicular to $\mathbf{B}_{\mathbf{0}}$ we have a spectral anisotropy $\Omega=90^{\circ}$, while for an isotropic spectrum $\Omega=45^{\circ}$. Numerical simulations in $2 \mathrm{D}$ configuration by Shebalin et al. (1983) confirmed the occurrence of anisotropy, and found that anisotropy increases with the Reynolds number. Unfortunately, in these old simulations, the Reynolds numbers used are too small to achieve a well defined spectral anisotropy. Carbone and Veltri (1990) started from the spectral equations obtained through the Direct Interaction Approximation closure by Veltri et al. (1982), and derived a shell model analogous for the anisotropic MHD turbulence. The phenomenological anisotropic spectrum obtained from the model, for both pseudo-energies obtained through polarizations $a=1,2$ defined through Equation (14), can be written as

$$
E_{a}^{ \pm}(\mathbf{k}, t) \sim C_{a}^{ \pm}\left[\ell_{\|}^{2} k_{\|}^{2}+\ell_{\perp}^{2} k_{\perp}^{2}\right]^{-\mu^{ \pm}} .
$$

Authors showed that spectral anisotropy is different within the three ranges of turbulence. Wave vectors perpendicular to $\mathbf{B}_{\mathbf{0}}$ are present in the spectrum, but when the process of energy transfer generates a strong anisotropy (at small times), a competing process takes place which redistributes the energy over all wave vectors. The dynamical balance between these tendencies fixes the value of the spectral anisotropy $\Omega \simeq 55^{\circ}$ in the inertial range. On the contrary, since the redistribution of energy cannot take place, in the dissipation domain the spectrum remains strongly anisotropic, with $\Omega \simeq 80^{\circ}$. When the Reynolds number increases, the contribution of the inertial range extends, 
and the increases of the total anisotropy tends to saturate at about $\Omega \simeq 60^{\circ}$ at Reynolds number of $10^{5}$. This value corresponds to a rather low value for the ratio between parallel and perpendicular correlation lengths $\ell_{\|} / \ell_{\perp} \simeq 2$, too small with respect to the observed value $\ell_{\|} / \ell_{\perp} \geq 10$. This suggests that the non-linear dynamical evolution of an initially isotropic spectrum of turbulence is perhaps not sufficient to explain the observed anisotropy. Recent numerical simulations confirmed these results (Oughton et al., 1994).

\subsubsection{Fluctuations correlation length and the Maltese Cross}

The correlation time, as defined in Appendix 12, estimates how much an element of our time series $x(t)$ at time $t_{1}$ depends on the value assumed by $x(t)$ at time $t_{0}$, being $t_{1}=t_{0}+\delta t$. This concept can be transferred from the time domain to the space domain if we adopt the Taylor hypothesis and, consequently, we can talk about spatial scales.

Correlation lengths in the solar wind generally increase with heliocentric distance (Matthaeus and Goldstein, 1982b; Bruno and Dobrowolny, 1986), suggesting that large scale correlations are built up during the wind expansion. This kind of evolution is common to both fast and slow wind as shown in Figure 25, where we can observe the behavior of the $B_{z}$ correlation function for fast and slow wind at 0.3 and $0.9 \mathrm{AU}$.

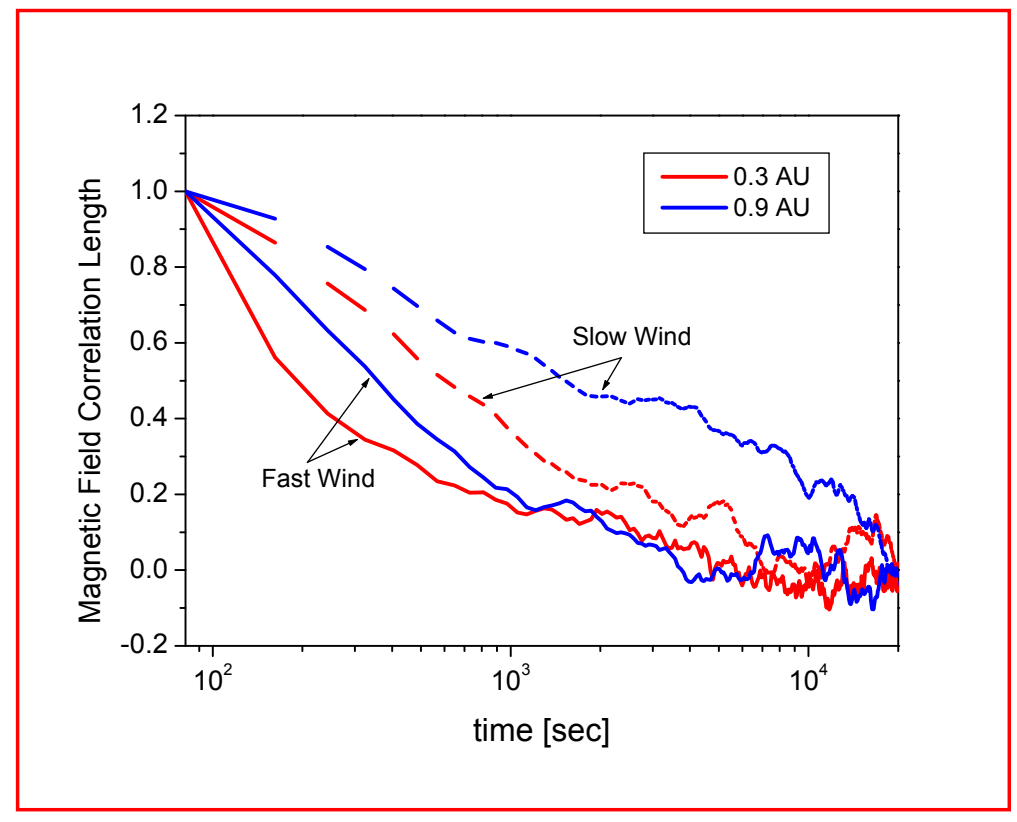

Figure 25: Correlation function just for the $Z$ component of interplanetary magnetic field as observed by Helios 2 during its primary mission to the Sun. The blue color refers to data recorded at $0.9 \mathrm{AU}$ while the red color refers to $0.3 \mathrm{AU}$. Solid lines refer to fast wind, dashed lines refer to slow wind.

Moreover, the fast wind correlation functions decrease much faster than those related to slow wind. This behavior reflects the fact that the stochastic character of Alfvénic fluctuations in the fast wind is very efficient in decorrelating the fluctuations of each of the magnetic field components.

More detailed studies performed by Matthaeus et al. (1990) provided for the first time the two-dimensional correlation function of solar wind fluctuations at $1 \mathrm{AU}$. The original dataset comprised approximately 16 months of almost continuous magnetic field $5-$ min averages. These

Living Reviews in Solar Physics

http://www. livingreviews .org/lrsp-2005-4 
results, based on ISEE 3 magnetic field data, are shown in Figure 26, also called the "The Maltese Cross".

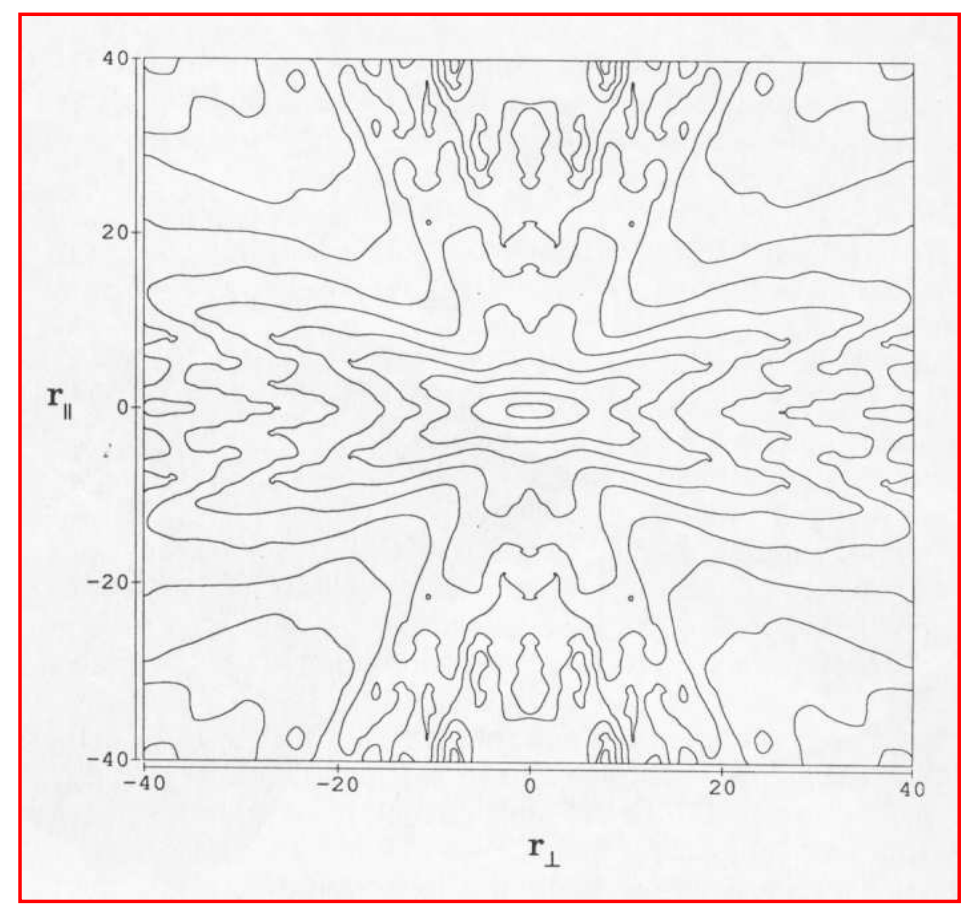

Figure 26: Contour plot of the 2D correlation function of interplanetary magnetic field fluctuations as a function of parallel and perpendicular distance with respect to the mean magnetic field. The separation in $r_{\|}$and $r_{\perp}$ is in units of $10^{10} \mathrm{~cm}$ (adopted from Matthaeus et al., 1990, (c) 1990 American Geophysical Union, reproduced by permission of American Geophysical Union).

This figure has been obtained under the hypothesis of cylindrical symmetry. Real determination of the correlation function could be obtained only in the positive quadrant, and the whole plot was then made by mirroring these results on the remaining three quadrants. The iso-contour lines show contours mainly elongated along the ambient field direction or perpendicular to it. Alfvénic fluctuations with $\mathbf{k} \| \mathbf{B}_{\mathbf{0}}$ contribute to contours elongated parallel to $r_{\perp}$. Fluctuations in the two-dimensional turbulence limit (Montgomery, 1982) contribute to contours elongated parallel to $r_{\|}$. This two-dimensional turbulence is characterized for having both the wave vector $\mathbf{k}$ and the perturbing field $\delta \mathbf{b}$ perpendicular to the ambient field $\mathbf{B}_{\mathbf{0}}$. Given the fact that the analysis did not select fast and slow wind, separately, it is likely that most of the slab correlations came from the fast wind while the $2 \mathrm{D}$ correlations came from the slow wind.

Anisotropic turbulence has been observed in laboratory plasmas and reverse pinch devices (Zweben et al., 1979), and has been studied both theoretically (Montgomery, 1982; Zank and Matthaeus, 1992) and through numerical simulations (Shebalin et al., 1983; Oughton, 1993). In particular, these simulations focused on non-linear spectral transfer within MHD turbulence in presence of a relevant mean magnetic field. They observed that a strong anisotropy is created during the turbulent process and much of the power is transferred to fluctuations with higher $\mathbf{k}_{\perp}$ and less to fluctuations with higher $\mathbf{k}_{\|}$. Bieber et al. (1996) formulated an observational test to distinguish the slab (Alfvénic) from the 2D component within interplanetary turbulence. These authors assumed a mixture of transverse fluctuations, some of which have wave vectors 
perpendicular $\mathbf{k} \perp \mathbf{B}_{\mathbf{0}}$ and polarization of fluctuations $\delta \mathbf{B}\left(\mathbf{k}_{\perp}\right)$ perpendicular to both vectors (2D geometry with $k_{\|} \simeq 0$ ), and some parallel to the mean magnetic field $\mathbf{k} \| \mathbf{B}_{\mathbf{0}}$, the polarization of fluctuations $\delta \mathbf{B}\left(k_{\|}\right)$being perpendicular to the direction of $\mathbf{B}_{\mathbf{0}}$ (slab geometry with $\mathbf{k}_{\perp} \simeq 0$ ). The magnetic field is then rotated into the same mean field coordinate system used by Belcher and Davis Jr (1971) and Belcher and Solodyna (1975), where the y-coordinate is perpendicular to both $\mathbf{B}_{\mathbf{0}}$ and the radial direction, while the x-coordinate is perpendicular to $\mathbf{B}_{\mathbf{0}}$ but with a component also in the radial direction. Using that geometry, and defining the power spectrum matrix as

$$
P_{i j}(\mathbf{k})=\frac{1}{(2 \pi)^{3}} \int d^{3} \mathbf{r}\left\langle\mathbf{B}_{i}(\mathbf{x}) \mathbf{B}_{\mathbf{j}}(\mathbf{x}+\mathbf{r})\right\rangle \mathbf{e}^{-\mathbf{i k} \cdot \mathbf{r}},
$$

it can be found that, assuming axisymmetry, a two-component model can be written in the frequency domain

$$
\begin{aligned}
& f P_{y y}(f)=r C_{s}\left(\frac{2 \pi f}{U_{w} \cos \psi}\right)^{1-q}+(1-r) C_{s} \frac{2 q}{(1+q)}\left(\frac{2 \pi f}{U_{w} \sin \psi}\right)^{1-q}, \\
& f P_{x x}(f)=r C_{s}\left(\frac{2 \pi f}{U_{w} \cos \psi}\right)^{1-q}+(1-r) C_{s} \frac{2}{(1+q)}\left(\frac{2 \pi f}{U_{w} \sin \psi}\right)^{1-q},
\end{aligned}
$$

where the anisotropic energy spectrum is the sum of both components:

$$
f T(f)=2 r C_{s}\left(\frac{2 \pi f}{U_{w} \cos \psi}\right)^{1-q}+2(1-r) C_{s}\left(\frac{2 \pi f}{U_{w} \sin \psi}\right)^{1-q} .
$$

Here $f$ is the frequency, $C_{s}$ is a constant defining the overall spectrum amplitude in wave vector space, $U_{w}$ is the bulk solar wind speed and $\psi$ is the angle between $\mathbf{B}_{\mathbf{0}}$ and the wind direction. Finally, $r$ is the fraction of slab components and $(1-r)$ is the fraction of $2 \mathrm{D}$ components.

The ratio test adopted by these authors was based on the ratio between the reduced perpendicular spectrum (fluctuations $\perp$ to the mean field and solar wind flow direction) and the reduced quasi-parallel spectrum (fluctuations $\perp$ to the mean field and in the plane defined by the mean field and the flow direction). This ratio, expected to be 1 for slab turbulence, resulted to be $\sim 1.4$ for fluctuations within the inertial range, consistent with $74 \%$ of $2 \mathrm{D}$ turbulence and $26 \%$ of slab. A further test, the anisotropy test, evaluated how the spectrum should vary with the angle between the mean magnetic field and the flow direction of the wind. The measured slab spectrum should decrease with the field angle while the 2D spectrum should increase, depending on how these spectra project on the flow direction. The results from this test were consistent with with $95 \%$ of $2 \mathrm{D}$ turbulence and $5 \%$ of slab. In other words, the slab turbulence due to Alfvénic fluctuations would be a minor component of interplanetary MHD turbulence. A third test derived from Mach number scaling associated with the nearly incompressible theory (Zank and Matthaeus, 1992), assigned the same fraction $\sim 80 \%$ to the $2 \mathrm{D}$ component. However, the data base for this analysis was derived from Helios magnetic measurements, and all data were recorded near times of solar energetic particle events. Moreover, the quasi totality of the data belonged to slow solar wind (Wanner and Wibberenz, 1993) and, as such, this analysis cannot be representative of the whole phenomenon of turbulence in solar wind. As a matter of fact, using Ulysses observations, Smith (2003) found that in the polar wind the percentage of slab and 2D components is about the same, say the high latitude slab component results unusually higher as compared with ecliptic observations.

Successive theoretical works by Ghosh et al. (1998a,b) in which they used compressible models in large variety of cases was able to obtain, in some cases, parallel and perpendicular correlations similar to those obtained in the solar wind. However, they concluded that the "Maltese" cross does not come naturally from the turbulent evolution of the fluctuations but it strongly depends

Living Reviews in Solar Physics

http://www. livingreviews . org/lrsp-2005-4 
on the initial conditions adopted when the simulation starts. It seems that individual existence of these correlations in the initial data represents an unavoidable constraint. Moreover, they also stressed the importance of time-averaging since the interaction between slab waves and transverse pressure-balanced magnetic structures causes the slab turbulence to evolve towards a state in which a two-component correlation function emerges during the process of time averaging.

The presence of two populations, i.e., a slab-like and a quasi-2D like, was also inferred by Dasso et al. (2003). These authors computed the reduced spectra of the normalized cross-helicity and the Alfvén ratio from ACE dataset. These parameters, calculated for different intervals of the angle $\theta$ between the flow direction and the orientation of the mean field $\mathbf{B}_{\mathbf{0}}$, showed a remarkable dependence on $\theta$.

The geometry used in these analyses assumes that the energy spectrum in the rest frame of the plasma is axisymmetric and invariant for rotations about the direction of $\mathbf{B}_{\mathbf{0}}$. Even if these assumption are good when we want to translate results coming from 2D numerical simulations to 3D geometry, these assumptions are quite in contrast with the observational fact that the eigenvalues of the variance matrix are different, namely $\lambda_{3} \neq \lambda_{2}$.

Going back from the correlation tensor to the power spectrum is a complicated technical problem. However, Carbone et al. (1995a) derived a description of the observed anisotropy in terms of a model for the three-dimensional energy spectra of magnetic fluctuations. The divergence-less of the magnetic field allows to decompose the Fourier amplitudes of magnetic fluctuations in two independent polarizations: The first one $I^{[1]}(\mathbf{k})$ corresponds, in the weak turbulence theory, to the Alfvénic mode, while the second polarization $I^{[2]}(\mathbf{k})$ corresponds to the magnetosonic mode. By using only the hypothesis that the medium is statistically homogeneous and some algebra, authors found that the energy spectra of both polarizations can be related to the two-points correlation tensor and to the variance matrix. Through numerical simulations (see later in the review) it has been shown that the anisotropic energy spectrum can be described in the inertial range by a phenomenological expression

$$
I^{[s]}(\mathbf{k})=C_{s}\left[\left(\ell_{x}^{[s]} k_{x}\right)^{2}+\left(\ell_{y}^{[s]} k_{y}\right)^{2}+\left(\ell_{z}^{[s]} k_{z}\right)^{2}\right]^{-1-\mu_{s} / 2},
$$

where $k_{i}$ are the Cartesian components of the wave vector $\mathbf{k}$, and $C_{s}, \ell_{i}^{[s]}$, and $\mu_{s}(s=1,2$ indicates both polarizations; $i=x, y, z)$ are free parameters. In particular, $C_{s}$ gives information on the energy content of both polarizations, $\ell_{i}^{[s]}$ represent the spectral extensions along the direction of a given system of coordinates, and $\mu_{s}$ are two spectral indices.

A fit to the eigenvalues of the variance matrix allowed Carbone et al. (1995a) to fix the free parameters of the spectrum for both polarizations. They used data from Bavassano et al. (1982a) who reported the values of $\lambda_{i}$ at five wave vectors calculated at three heliocentric distances, selecting periods of high correlation (Alfvénic periods) using magnetic field measured by the Helios 2 spacecraft. They found that the spectral indices of both polarizations, in the range $1.1 \leq \mu_{1} \leq 1.3$ and $1.46 \leq \mu_{2} \leq 1.8$ increase systematically with increasing distance from the Sun, the polarization [2] spectra are always steeper than the corresponding polarization [1] spectra, while polarization [1] is always more energetic than polarization [2]. As far as the characteristic lengths are concerned, it can be found that $\ell_{x}^{[1]}>\ell_{y}^{[1]} \gg \ell_{z}[1]$, indicating that wave vectors $\mathbf{k} \| \mathbf{B}_{0}$ largely dominate. Concerning polarization [2], it can be found that $\ell_{x}[2] \gg \ell_{y}^{[2]} \simeq \ell_{z}^{[2]}$, indicating that the spectrum $I^{[2]}(\mathbf{k})$ is strongly flat on the plane defined by the directions of $\mathbf{B}_{\mathbf{0}}$ and the radial direction. Within this plane, the energy distribution does not present any relevant anisotropy.

Let us compare these results with those by Matthaeus et al. (1990), the comparison being significant as far as the plane $y z$ is taken into account. The decomposition of Carbone et al. (1995a) in two independent polarizations is similar to that of Matthaeus et al. (1990), a contour plot of the trace of the correlation tensor Fourier transform $T(\mathbf{k})=I^{[1]}(\mathbf{k})+I^{[2]}(\mathbf{k})$ on the plane $\left(k_{y} ; k_{z}\right)$ 
shows two populations of fluctuations, with wave vectors nearly parallel and nearly perpendicular to $\mathbf{B}_{\mathbf{0}}$, respectively. The first population is formed by all the polarization [1] fluctuations and by the fluctuations with $\mathbf{k} \| \mathbf{B}_{\mathbf{0}}$ belonging to polarization [2]. The latter fluctuations are physically indistinguishable from the former, in that when $\mathbf{k}$ is nearly parallel to $\mathbf{B}_{\mathbf{0}}$, both polarization vectors are quasi-perpendicular to $\mathbf{B}_{\mathbf{0}}$. On the contrary, the second population is almost entirely formed by fluctuations belonging to polarization [2]. While it is clear that fluctuations with $\mathbf{k}$ nearly parallel to $\mathbf{B}_{\mathbf{0}}$ are mainly polarized in the plane perpendicular to $\mathbf{B}_{\mathbf{0}}$ (a consequence of $\nabla \cdot \mathbf{B}=\mathbf{0}$ ), fluctuations with $\mathbf{k}$ nearly perpendicular to $\mathbf{B}_{\mathbf{0}}$ are polarized nearly parallel to $\mathbf{B}_{\mathbf{0}}$.

Although both models yield to the occurrence of two populations, Matthaeus et al. (1990) give an interpretation of their results, which is in contrast with that of Carbone et al. (1995a). Namely Matthaeus et al. (1990) suggest that a nearly 2D incompressible turbulence characterized by wave vectors and magnetic fluctuations, both perpendicular to $\mathbf{B}_{\mathbf{0}}$, is present in the solar wind. However, this interpretation does not arise from data analysis, rather from the 2D numerical simulations by Shebalin et al. (1983) and of analytical studies (Montgomery, 1982). Let us note, however, that in the former approach, which is strictly $2 \mathrm{D}$, when $\mathbf{k} \perp \mathbf{B}_{\mathbf{0}}$ magnetic fluctuations are necessarily parallel to $\mathbf{B}_{\mathbf{0}}$. In the latter one, along with incompressibility, it is assumed that the energy in the fluctuations is much less than in the DC magnetic field; both hypotheses do not apply to the solar wind case. On the contrary, results by Carbone et al. (1995a) can be directly related to the observational data. To conclude, it is worth reporting that a model like that discussed here, that is a superposition of fluctuations with both slab and $2 \mathrm{D}$ components, has been used to describe turbulence in the Jovian magnetosphere (Saur et al., 2002, 2003).

\subsubsection{Magnetic helicity}

Magnetic helicity $H_{\mathrm{m}}$, as defined in Appendix 13.1, measures the "knottedness" of magnetic field lines (Moffat, 1978). Moreover, $H_{\mathrm{m}}$ is a pseudo scalar and changes sign for coordinate inversion. The plus or minus sign, for circularly polarized magnetic fluctuations in a slab geometry, indicates right or left hand polarization. The general features of the magnetic helicity spectrum in the solar wind were for the first time described by Matthaeus and Goldstein (1982b) in the outer heliosphere, and by Bruno and Dobrowolny (1986) in the inner heliosphere. A useful dimensionless way to represent both the degree of and the sense of polarization is the normalized magnetic helicity $\sigma_{\mathrm{m}}$ (see Appendix 13.1). This quantity can randomly vary between +1 and -1 , as shown in Figure 27 from the work by Matthaeus and Goldstein (1982b) and relative to Voyager's data taken at 1 AU. However, net values of \pm 1 are reached only for pure circularly polarized waves.

Based on these results, Goldstein et al. (1991) were able to reproduce the distribution of the percentage of occurrence of values of $\sigma_{\mathrm{m}}(f)$ adopting a model where the magnitude of the magnetic field was allowed to vary in a random way and the tip of the vector moved near a sphere. By this way they showed that the interplanetary magnetic field helicity measurements were inconsistent with the previous idea that fluctuations were randomly circularly polarized at all scales and were also magnitude preserving.

However, evidence for circular polarized MHD waves in the high frequency range was provided by Polygiannakis et al. (1994), who studied interplanetary magnetic field fluctuations from various datasets at various distances ranging from 1 to 20 AU. They also concluded that the difference between left and right hand polarizations is significant and continuously varying.

As already noticed by Smith et al. $(1983,1984)$, knowing the sign of $\sigma_{\mathrm{m}}$ and the sign of the normalized cross-helicity $\sigma_{\mathrm{c}}$ it is possible to infer the sense of polarization of the fluctuations. As a matter of fact, a positive cross-helicity indicates an Alfvén mode propagating outward, while a negative cross-helicity indicates a mode propagating inward. On the other hand, we know that a positive magnetic-helicity indicates a right hand polarized mode, while a negative magnetichelicity indicates a left hand polarized mode. Thus, since the sense of polarization depends on

Living Reviews in Solar Physics

http://www. livingreviews . org/lrsp-2005-4 


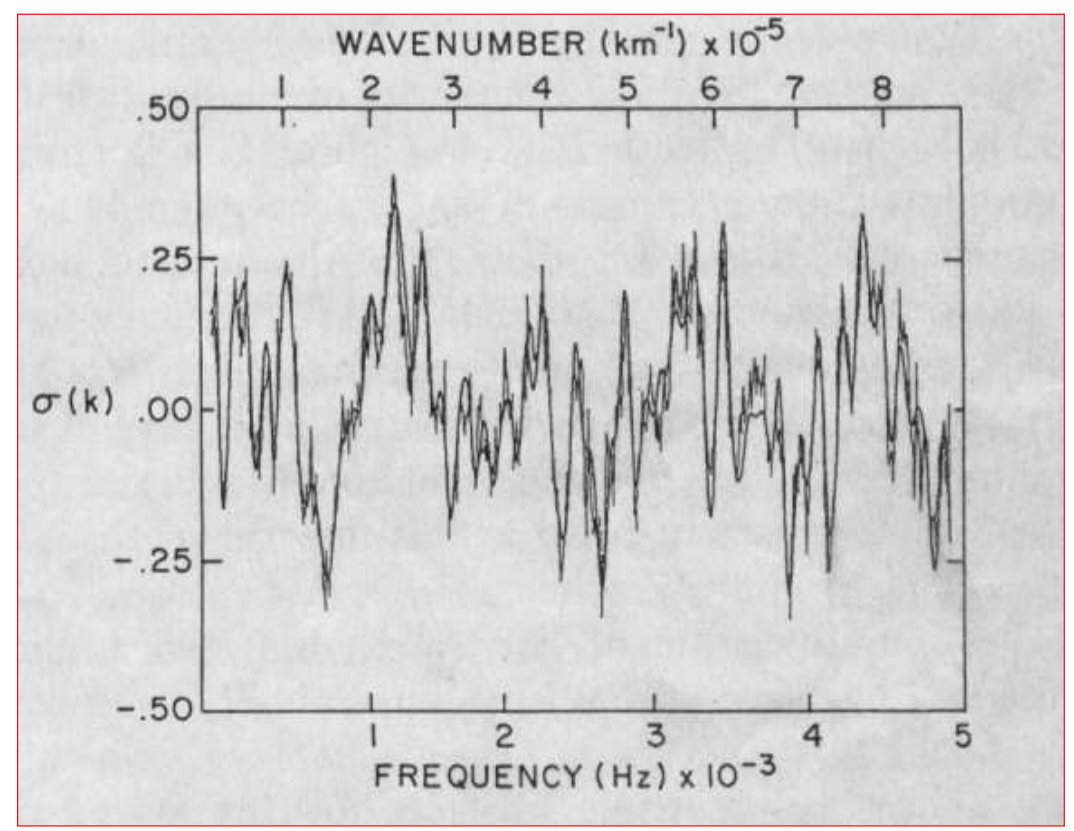

Figure 27: $\sigma_{\mathrm{m}}$ vs. frequency and wave number relative to an interplanetary data sample recorded by Voyager 1 at approximately 1 AU (adopted from Matthaeus and Goldstein, 1982b, (c) 1982 American Geophysical Union, reproduced by permission of American Geophysical Union).

the propagating direction with respect to the observer, $\sigma_{\mathrm{m}}(f) \sigma_{\mathrm{c}}(f)<0$ will indicate right circular polarization while $\sigma_{\mathrm{m}}(f) \sigma_{\mathrm{c}}(f)>0$ will indicate left circular polarization. Thus, any time magnetic helicity and cross-helicity are available from measurements in a super-Alfvénic flow, it is possible to infer the rest frame polarization of the fluctuations from a single point measurements, assuming the validity of the slab geometry.

The high variability of $\sigma_{\mathrm{m}}$, observable in Voyager's data (see Figure 27), was equally observed in Helios 2 data in the inner heliosphere (Bruno and Dobrowolny, 1986). The authors of this last work computed the difference $(M H>0)-|M H<0|$ of magnetic helicity for different frequency bands and noticed that most of the resulting magnetic helicity was contained in the lowest frequency band. This result supported the theoretical prediction of an inverse cascade of magnetic helicity from the smallest to the largest scales during turbulence development (Pouquet et al., 1976).

Numerical simulations of the incompressible MHD equations by Mininni et al. (2003a), discussed in Section 3.1.8, clearly show the tendency of magnetic helicity to follow an inverse cascade. These authors injected a weak magnetic field at small scales in a system kept in a stationary regime of hydrodynamic turbulence and followed the exponential growth of magnetic energy due to the dynamo action. This evolution can be seen in Figure 28 in the same format described for Figure 33, shown in Section 3.1.8. Now, the forcing is applied at wave number $k_{\text {force }}=10$ in order to give enough room for the inverse cascade to develop. The fluid is initially in a strongly turbulent regime as a result of the action of the external force at wave number $k_{\text {force }}=10$. An initial magnetic fluctuation is introduced at $t=0$ at $k_{\text {seed }}=35$. The magnetic energy starts growing exponentially fast and, when the saturation is reached, the magnetic energy is larger than the kinetic energy. Notably, it is much larger at the largest scales of the system (i.e., $k=1$ ). At these large scales, the system is very close to a magnetostatic equilibrium characterized by a force-free configuration. 


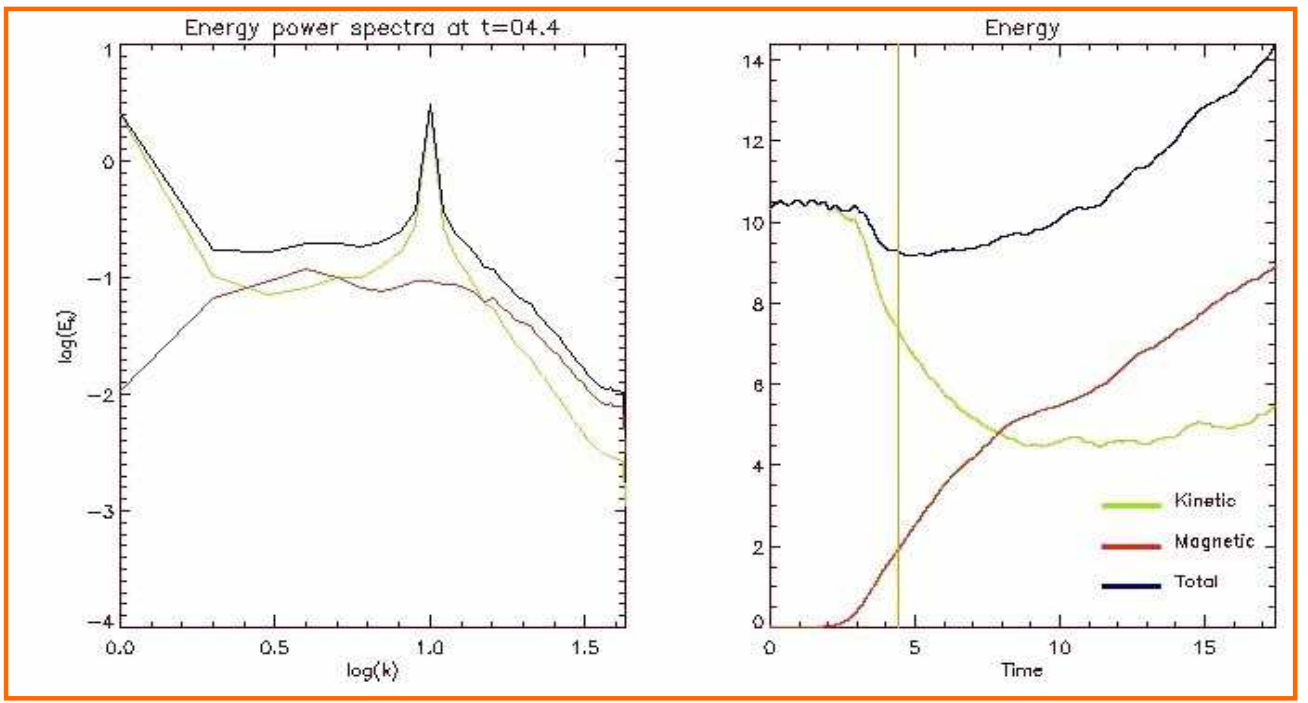

Figure 28: Still from a Numerical simulation of the incompressible MHD equations in three dimensions, assuming periodic boundary conditions (see details in Mininni et al., 2003a). The left panel shows the power spectra for kinetic energy (green), magnetic energy (red), and total energy (blue) vs. time. The right panel shows the spatially integrated kinetic, magnetic, and total energies vs. time. The vertical (orange) line indicates the current time. These results correspond to a $128^{3}$ simulation with an external force applied at wave number $k_{\text {force }}=10$ (movie kindly provided by D. Gómez). (To watch the movie, please go to the online version of this review article at http: //www.livingreviews.org/lrsp-2005-4.) 


\subsubsection{Alfvénic correlations as uncompressive turbulence}

In a famous paper, Belcher and Davis Jr (1971) showed that a strong correlation exists between velocity and magnetic field fluctuations, in the form

$$
\delta \mathbf{v} \simeq \pm \frac{\delta \mathbf{B}}{\sqrt{4 \pi \rho}},
$$

where the sign of the correlation is given by the $\operatorname{sign}\left[-\mathbf{k} \cdot \mathbf{B}_{\mathbf{0}}\right]$, being $\mathbf{k}$ the wave vector and $\mathbf{B}_{\mathbf{0}}$ the background magnetic field vector. These authors showed that in about $25 \mathrm{~d}$ of data from Mariner 5, out of the $160 \mathrm{~d}$ of the whole mission, fluctuations were described by Equation (36), and the sign of the correlation was such to indicate always an outward sense of propagation with respect to the Sun. Authors also noted that these periods mainly occur within the trailing edges of high-speed streams. Moreover, in the regions where Equation (36) is verified to a high degree, the magnetic field magnitude is almost constant $\left(B^{2} \sim\right.$ const.).

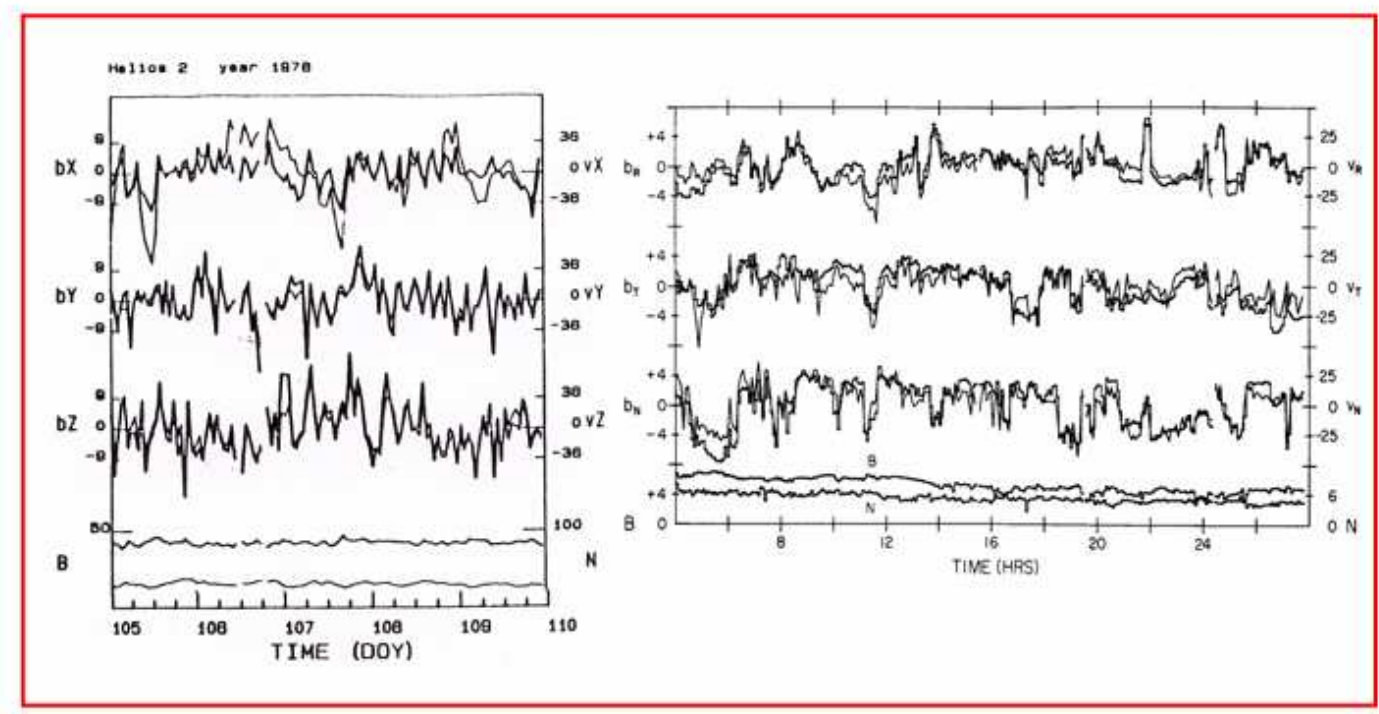

Figure 29: Alfvénic correlation in fast solar wind. Left panel: large scale Alfvénic fluctuations found by Bruno et al. (1985). Right panel: small scale Alfvénic fluctuations for the first time found by Belcher and Solodyna (1975) (c) 1975, 1985 American Geophysical Union, reproduced and adapted by permission of American Geophysical Union).

Today we know that Alfvénic correlations are ubiquitous in the solar wind and that these correlations are much stronger and are found at lower and lower frequencies, as we look at shorter and shorter heliocentric distances. In the right panel of Figure 29 we show results from Belcher and Solodyna (1975) obtained on the basis of 5 min averages of velocity and magnetic field recorded by Mariner 5 in 1967, during its mission to Venus. On the left panel of Figure 29 we show results from a similar analysis performed by Bruno et al. (1985) obtained on the basis of $1 \mathrm{~h}$ averages of velocity and magnetic field recorded by Helios 2 in 1976, when the s/c was at 0.29 AU from the Sun. These last authors found that, in their case, Alfvénic correlations extended to time periods as low as $15 \mathrm{~h}$ in the s/c frame at $0.29 \mathrm{AU}$, and to periods a factor of two smaller near the Earth's orbit. Now, if we think that this long period of the fluctuations at $0.29 \mathrm{AU}$ was larger than the transit time from the Sun to the s/c, this results might be the first evidence for a possible solar 
origin for these fluctuations, probably caused by the shuffling of the foot-points of the solar surface magnetic field.

Alfvén modes are not the only low frequency plasma fluctuations allowed by the MHD equations but they certainly are the most frequent fluctuations observed in the solar wind. The reason why other possible propagating modes like the slow sonic mode and the fast magnetosonic mode cannot easily be found, depends on the fact that these compressive modes are strongly damped in the solar wind shortly after they are generated (see Section 6). On the contrary, Alfvénic fluctuations, which are difficult to be damped because of their uncompressive nature, survive much longer and dominate solar wind turbulence. Nevertheless, there are regions where Alfvénic correlations are much stronger like the trailing edge of fast streams, and regions where these correlations are weak like intervals of slow wind (Belcher and Davis Jr, 1971; Belcher and Solodyna, 1975). However, the degree of Alfvénic correlations unavoidably fades away with increasing heliocentric distance, although it must be reported that there are cases when the absence of strong velocity shears and compressive phenomena favor a high Alfvénic correlation up to very large distances from the Sun (Roberts et al., 1987a; see Section 5.1).

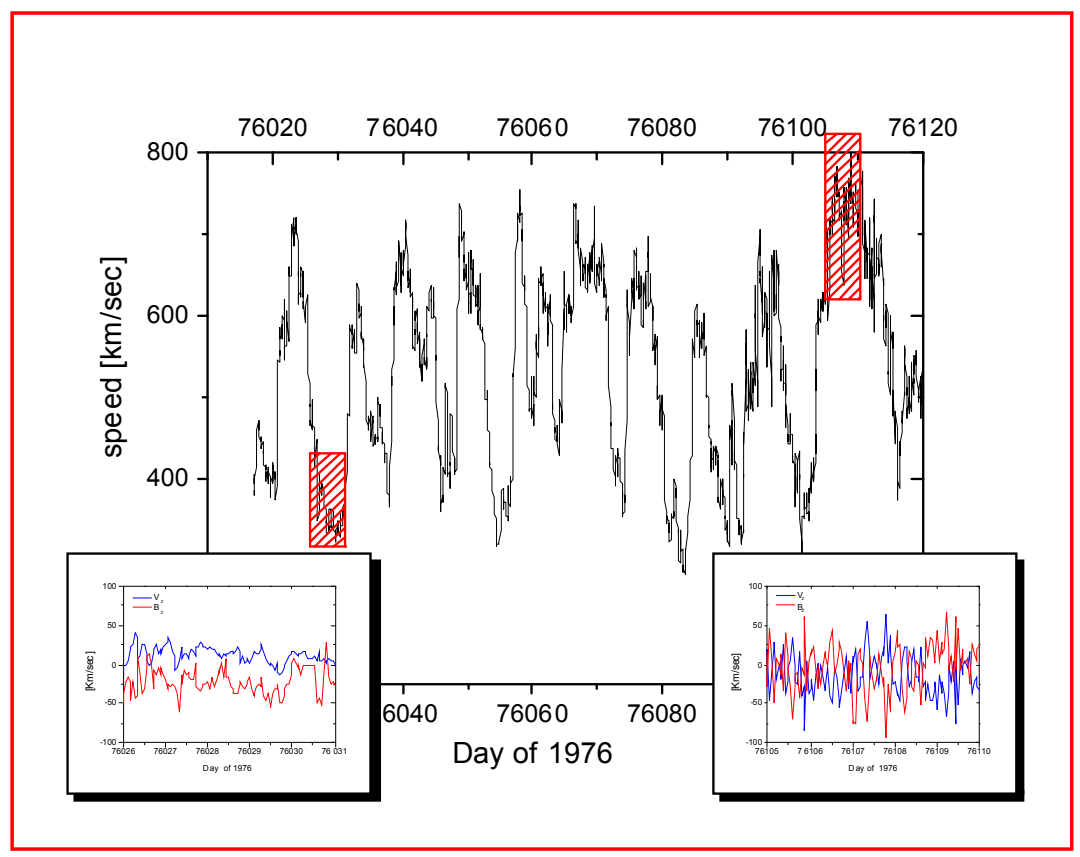

Figure 30: Alfvénic correlation in fast and slow wind. Notice the different degree of correlation between these two types of wind.

Just to give a qualitative quick example about Alfvénic correlations in fast and slow wind, we show in Figure 30 the speed profile for about $100 \mathrm{~d}$ of 1976 as observed by Helios 2, and the traces of velocity and magnetic field $Z$ components (see Appendix 15 for the orientation of the reference system) $V_{Z}$ and $B_{Z}$ (this last one expressed in Alfvén units, see Appendix 13.1) for two different time intervals, which have been enlarged in the two inserted small panels. The high velocity interval shows a remarkable anti-correlation which, since the mean magnetic field $\mathbf{B}_{\mathbf{0}}$ is oriented away from the Sun, suggests a clear presence of outward oriented Alfvénic fluctuations given that the sign of the correlation is the $\operatorname{sign}\left[-\mathbf{k} \cdot \mathbf{B}_{\mathbf{0}}\right]$. At odds with the previous interval, the slow wind shows that the two traces are rather uncorrelated. For sake of brevity, we omit to show

Living Reviews in Solar Physics

http: //www . livingreviews . org/lrsp-2005-4 
the very similar behavior for the other two components, within both fast and slow wind.

The discovery of Alfvénic correlations in the solar wind stimulated fundamental remarks by Kraichnan (1974) who, following previous theoretical works by Kraichnan (1965) and Iroshnikov (1963), showed that the presence of a strong correlation between velocity and magnetic fluctuations renders non-linear transfer to small scales less efficient than for the Navier-Stokes equations, leading to a turbulent behavior which is different from that described by Kolmogorov (1941). In particular, when Equation (36) is exactly satisfied, non-linear interactions in MHD turbulent flows cannot exist. This fact introduces a problem in understanding the evolution of MHD turbulence as observed in the interplanetary space. Both a strong correlation between velocity and magnetic fluctuations and a well defined turbulence spectrum (Figures 23, 30) are observed, and the existence of the correlations is in contrast with the existence of a spectrum which in turbulence is due to a non-linear energy cascade. Dobrowolny et al. (1980b) started to solve the puzzle on the existence of Alfvénic turbulence, say the presence of predominately outward propagation and the fact that MHD turbulence with the presence of both Alfvénic modes present will evolve towards a state where one of the mode disappears. However, a lengthy debate based on whether the highly Alfvénic nature of fluctuations is what remains of the turbulence produced at the base of the corona or the solar wind itself is an evolving turbulent magnetofluid, has been stimulating the scientific community for quite a long time.

\subsubsection{Radial evolution of Alfvénic turbulence}

The degree of correlation not only depends on the type of wind we look at, i.e., fast or slow, but also on the radial distance from the Sun and on the time scale of the fluctuations.

Figure 31 shows the radial evolution of $\sigma_{\mathrm{c}}$ (see Appendix 13.1) as observed by Helios and Voyager s/c (Roberts et al., 1987b). It is clear enough that $\sigma_{\mathrm{c}}$ not only tends to values around 0 as the heliocentric distance increases, but larger and larger time scales are less and less Alfvénic. Values of $\sigma_{\mathrm{c}} \sim 0$ suggest a comparable amount of "outward" and "inward" correlations.

The radial evolution affects also the Alfvén ratio $r_{\mathrm{A}}$ (see Appendix 13.3.1) as it was found by Bruno et al. (1985). However, early analyses (Belcher and Davis Jr, 1971; Solodyna and Belcher, 1976; Matthaeus and Goldstein, 1982b) had already shown that this parameter is usually less than unit. Spectral studies by Marsch and Tu (1990a), reported in Figure 32, showed that within slow wind it is the lowest frequency range the one that experiences the strongest decrease with distance, while the highest frequency range remains almost unaffected. Moreover, the same study showed that, within fast wind, the whole frequency range experiences a general depletion. The evolution is such that close to $1 \mathrm{AU}$ the value of $r_{\mathrm{A}}$ in fast wind approaches that in slow wind.

Moreover, comparing these results with those by Matthaeus and Goldstein (1982b) obtained from Voyager at 2.8 AU, it seems that the evolution recorded within fast wind tends to a sort of limit value around $0.4-0.5$.

Also Roberts et al. (1990), analysing fluctuations between $9 \mathrm{~h}$ and $3 \mathrm{~d}$ found a similar radial trend. These authors showed that $r_{\mathrm{A}}$ dramatically decreases from values around unit at the Earth's orbit towards $0.4-0.5$ at approximately $8 \mathrm{AU}$. For larger heliocentric distances, $r_{\mathrm{A}}$ seems to stabilize around this last value.

The reason why $r_{\mathrm{A}}$ tends to a value less than unit is still an open question although MHD computer simulations (Matthaeus, 1986) showed that magnetic reconnection and high plasma viscosity can produce values of $r_{\mathrm{A}}<1$ within the inertial range. Moreover, as pointed out by Grappin et al. (1991), the magnetic energy excess can be explained as a competing action between the "Alfvén effect" (Kraichnan, 1965), which would work towards equipartition, and the non-linear terms (Grappin et al., 1983). However, this argument forecasts an Alfvén ratio $r_{\mathrm{A}} \neq 1$ but, it does not say whether it would be larger or smaller than "1", i.e., we could also have a final excess of kinetic energy. 


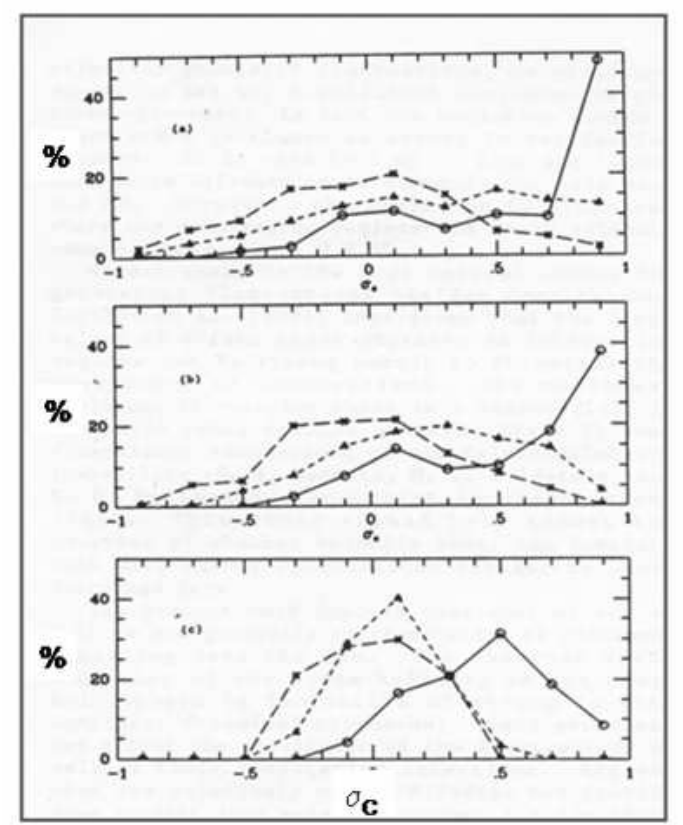

Figure 31: Histograms of normalized cross-helicity $\sigma_{\mathrm{c}}$ showing its evolution between 0.3 (circles), 2 (triangles), and 20 (squares) AU for different time scales: $3 \mathrm{~h}$ (top panel), $9 \mathrm{~h}$ (middle panel), and $81 \mathrm{~h}$ (bottom panel) (adopted from Roberts et al., 1987b, (c) 1987 American Geophysical Union, reproduced by permission of American Geophysical Union).

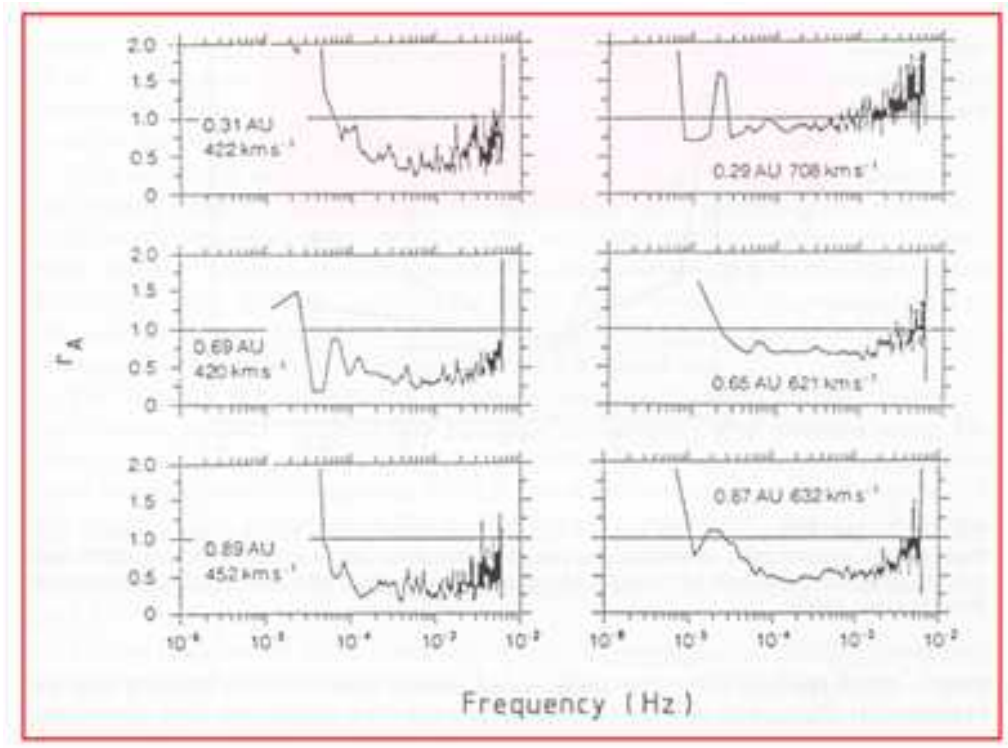

Figure 32: Values of the Alfvén ratio $r_{\mathrm{A}}$ as a function of frequency and heliocentric distance, within slow (left column) and fast (right column) wind (adopted from Marsch and Tu, 1990a, (c) 1990 American Geophysical Union, reproduced by permission of American Geophysical Union). 
Similar unbalance between magnetic and kinetic energy has recently been found in numerical simulations by Mininni et al. (2003a), already cited in Section 3.1.6. These authors studied the effect of a weak magnetic field at small scales in a system kept in a stationary regime of hydrodynamic turbulence. In these conditions, the dynamo action causes the initial magnetic energy to grow exponentially towards a state of quasi equipartition between kinetic and magnetic energy. This simulation was aiming to provide more insights on a microscopic theory of the alpha-effect, which is responsible to convert part of the toroidal magnetic field on the Sun back to poloidal to sustain the cycle. However, when the simulation saturates, the unbalance between kinetic and magnetic energy reminds the conditions in which the Alfvén ratio is found in interplanetary space. Results from the above study can be viewed in the animation of Figure 33. At very early time the fluid is in a strongly turbulent regime as a result of the action of the external force at wave number $k_{\text {force }}=3$. An initial magnetic fluctuation is introduced at $\mathrm{t}=0$ at $k_{\text {seed }}=35$. The magnetic energy starts growing exponentially fast and, when the simulation reaches the saturation stage, the magnetic power spectrum exceeds the kinetic power spectrum at large wave numbers (i.e., $k>k_{\text {force }}$ ), as also observed in Alfvénic fluctuations of the solar wind (Bruno et al., 1985; Tu and Marsch, 1990a) as an asymptotic state (Roberts et al., 1987a,b; Bavassano et al., 2000b) of turbulence.

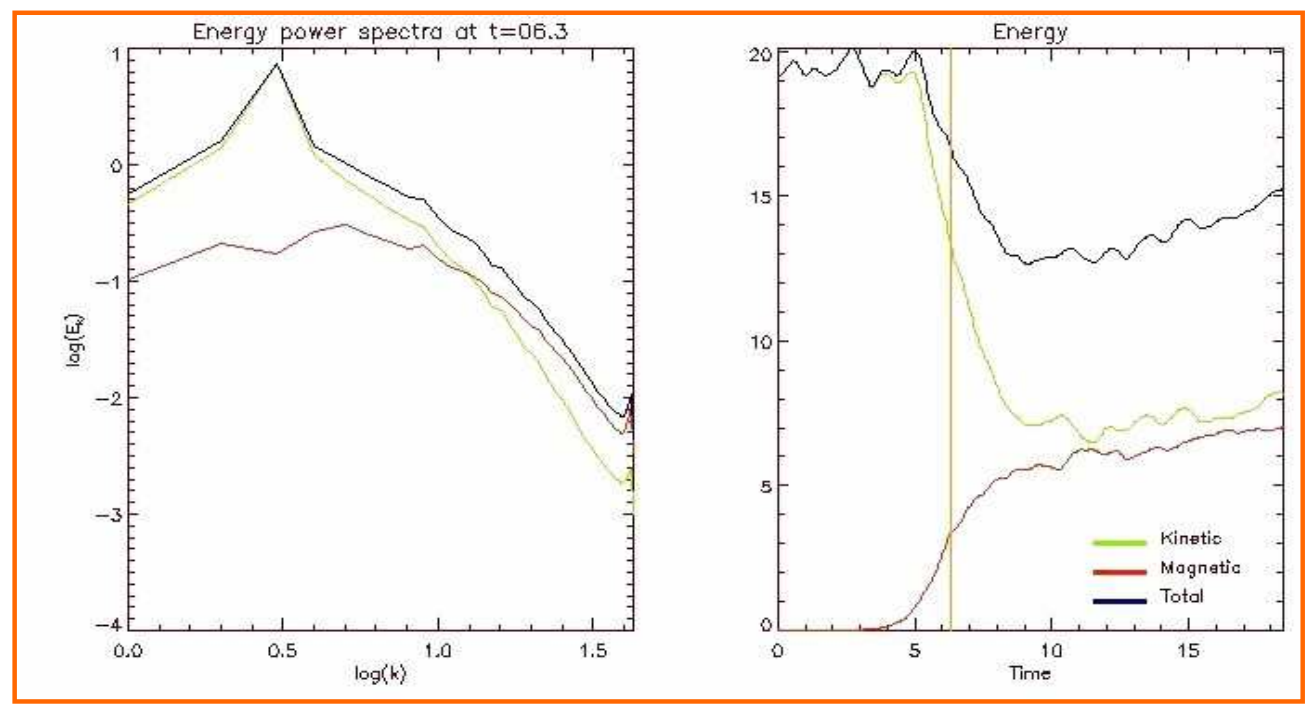

Figure 33: Still from a $128^{3}$ numerical simulation, as in Figure 28, but with an external force applied at wave number $k_{\text {force }}=3$ (movie kindly provided by D. Gómez). (To watch the movie, please go to the online version of this review article at http://www.livingreviews.org/lrsp-2005-4.)

However, when the two-fluid effect, such as the Hall current and the electron pressure (Mininni et al., 2003b), is included in the simulation, the dynamo can work more efficiently and the final stage of the simulation is towards equipartition between kinetic and magnetic energy.

On the other hand, Marsch and Tu (1993a) analyzed several intervals of interplanetary observations to look for a linear relationship between the mean electromotive force $\varepsilon=\langle\delta \mathbf{V} \delta \mathbf{B}\rangle$, generated by the turbulent motions, and the mean magnetic field $B_{0}$, as predicted by simple dynamo theory (Krause and Rädler, 1980). Although sizable electromotive force was found in interplanetary fluctuations, these authors could not establish any simple linear relationship between $B_{0}$ and $\varepsilon$.

Lately, Bavassano and Bruno (2000) performed a three-fluid analysis of solar wind Alfvénic fluctuations in the inner heliosphere, in order to evaluate the effect of disregarding the multi- 
fluid nature of the wind on the factor relating velocity and magnetic field fluctuations. It is well known that converting magnetic field fluctuations into Alfvén units we divide by the factor $F_{p}=\left(4 \pi M_{p} N_{p}\right)^{1 / 2}$. However, fluctuations in velocity tend to be smaller than fluctuations in Alfvén units. In Figure 34 we show scatter plots between the z-component of the Alfvén velocity and the proton velocity fluctuations. The $z$-direction has been chosen as the same of $\mathbf{V}_{\mathbf{p}} \times \mathbf{B}$, where $\mathbf{V}_{\mathbf{p}}$ is the proton bulk flow velocity and $\mathbf{B}$ is the mean field direction. The reason for such a choice is due to the fact that this direction is the least affected by compressive phenomena deriving from the wind dynamics. These results show that although the correlation coefficient in both cases is around -0.95 , the slope of the best fit straight line passes from 1 at 0.29 AU to a slope considerably different from 1 at 0.88 AU.

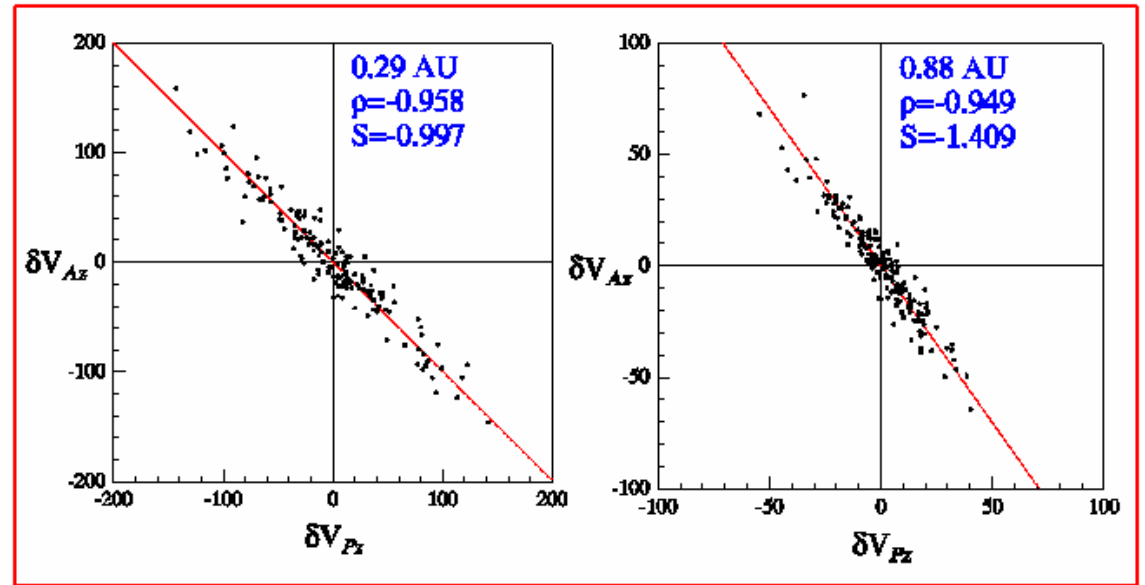

Figure 34: Scatter plot between the z-component of the Alfvén velocity and the proton velocity fluctuations at about $2 \mathrm{mHz}$. Data refer to Helios 2 observations at $0.29 \mathrm{AU}$ (left panel) and 0.88 AU (right panel) (adapted from Bavassano and Bruno, 2000; (c) 2000 American Geophysical Union, reproduced by permission of American Geophysical Union).

Belcher and Davis Jr (1971) suggested that this phenomenon had to be ascribed to the presence of $\alpha$ particles and to an anisotropy in the thermal pressure. Moreover, taking into account the multi-fluid nature of the solar wind, the dividing factor should become $F=F_{p} F_{i} F_{a}$, where $F_{i}$ would take into account the presence of other species besides protons, and $F_{a}$ would take into account the presence of pressure anisotropy $P_{\|} \neq P_{\perp}$, where $\|$ and $\perp$ refer to the background field direction. In particular, following Bavassano and Bruno (2000), the complete expressions for $F_{i}$ and $F_{a}$ are

$$
F_{i}=\left[1+\sum_{s}\left(M_{s} N_{s}\right) /\left(M_{p} N_{p}\right)\right]^{1 / 2}
$$

and

$$
F_{a}=\left[1-\frac{4 \pi}{B_{0}^{2}} \sum_{s}\left(P_{\| s}-P_{\perp s}+M_{s} N_{s} \mathbf{U}_{s}^{2}\right)\right]^{-1 / 2},
$$

where the letter "s" stands for the $s$-th species, being $\mathbf{U}_{s}=\mathbf{V}_{s}-\mathbf{V}$ its velocity in the center of mass frame of reference. $\mathbf{V}_{s}$ is the velocity of the species "s" in the s/c frame and $\mathbf{V}=$ $\left(\sum_{s} M_{s} N_{s} \mathbf{V}_{s}\right) /\left(\sum_{s} M_{s} N_{s}\right)$ is the velocity of the center of mass.

Bavassano and Bruno (2000) analyzed several time intervals within the same corotating high velocity stream observed at 0.3 and $0.9 \mathrm{AU}$ and performed the analysis using the new factor "F" to

Living Reviews in Solar Physics

http://www. livingreviews . org/lrsp-2005-4 
express magnetic field fluctuations in Alfvén units, taking into account the presence of $\alpha$ particles and electrons, besides the protons. However, the correction resulted to be insufficient to bring back to "1" the slope of the $\delta V_{\mathrm{P} z}-\delta V_{\mathrm{A} z}$ relationship shown in the right panel of Figure 34. In conclusion, the radial variation of the Alfvén ratio $r_{\mathrm{A}}$ towards values less than 1 is not completely due to a missed inclusion of multi-fluid effects in the conversion from magnetic field to Alfvén units. Thus, we are left with the possibility that the observed depletion of $r_{\mathrm{A}}$ is due to a natural evolution of turbulence towards a state in which magnetic energy becomes dominant (Grappin et al., 1991; Roberts et al., 1992; Roberts, 1992), as observed in the animation of Figure 33 taken from numerical simulations by Mininni et al. (2003a) or, it is due to the increased presence of magnetic structures like MFDT (Tu and Marsch, 1993).

\subsection{Turbulence studied via Elsässer variables}

The Alfvénic character of solar wind fluctuations,especially within corotating high velocity streams, suggests to use the Elsässer variables (Appendix 13.3) to separate the "outward" from the "inward" contribution to turbulence. These variables, used in theoretical studies by Dobrowolny et al. (1980a), Dobrowolny et al. (1980b), Veltri et al. (1982), Marsch and Mangeney (1987), and Zhou and Matthaeus (1989), were for the first time used in interplanetary data analysis by Grappin et al. (1990) and Tu et al. (1989b). In the following, we will describe and discuss several differences between "outward" and "inward" modes, but the most important one is about their origin. As a matter of fact, the existence of the Alfvénic critical point implies that only "outward" propagating waves of solar origin will be able to escape from the Sun. "Inward" waves, being faster than the wind bulk speed, will precipitate back to the Sun if they are generated before this point. The most important implication due to this scenario is that "inward" modes observed beyond the Alfvénic point cannot have a solar origin but they must have been created locally by some physical process. Obviously, for the other Alfvénic component, both solar and local origins are still possible.

\subsubsection{Ecliptic scenario}

Early studies by Belcher and Davis Jr (1971), performed on magnetic field and velocity fluctuations recorded by Mariner 5 during its trip to Venus in 1967, already suggested that the majority of the Alfvénic fluctuations are characterized by an "outward" sense of propagation, and that the best regions where to observe these fluctuations are the trailing edge of high velocity streams. Moreover, Helios spacecraft, repeatedly orbiting around the Sun between 0.3 to $1 \mathrm{AU}$, gave the first and unique opportunity to study the radial evolution of turbulence (Bavassano et al., 1982b; Denskat and Neubauer, 1983). Successively, when Elsässer variables were introduced in the analysis (Grappin et al., 1989), it was finally possible not only to evaluate the "inward" and "outward" Alfvénic contribution to turbulence but also to study the behavior of these modes as a function of the wind speed and radial distance from the Sun.

Figure 35 (Tu et al., 1990) clearly shows the behavior of $e^{ \pm}$(see Appendix 13.3) across a high speed stream observed at $0.3 \mathrm{AU}$. Within fast wind $e^{+}$is much higher than $e^{-}$and its spectral slope shows a break. Lower frequencies have a flatter slope while the slope of higher frequencies is closer to a Kolmogorov-like. $e^{-}$has a similar break but the slope of lower frequencies follows the Kolmogorov slope, while higher frequencies form a sort of plateau.

This configuration vanishes when we pass to the slow wind where both spectra have almost equivalent power density and follow the Kolmogorov slope. This behavior, for the first time reported by Grappin et al. (1990), is commonly found within corotating high velocity streams, although much more clearly expressed at shorter heliocentric distances, as shown below.

Spectral power associated with outward (right panel) and inward (left panel) Alfvénic fluctuations, based on Helios 2 observations in the inner heliosphere, are concisely reported in Figure 36. 


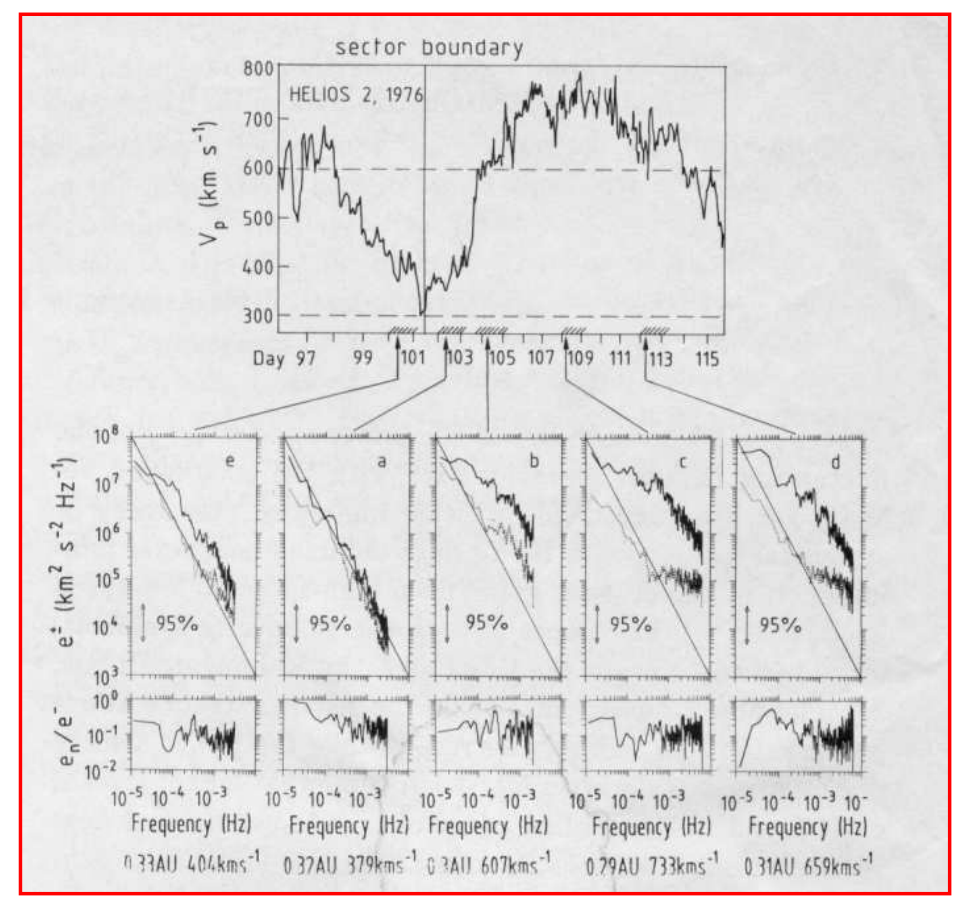

Figure 35: Power density spectra $e^{ \pm}$computed from $\delta z^{ \pm}$fluctuations for different time intervals indicated by the arrows (adopted from Tu et al., 1990, (c) 1990 American Geophysical Union, reproduced by permission of American Geophysical Union). 


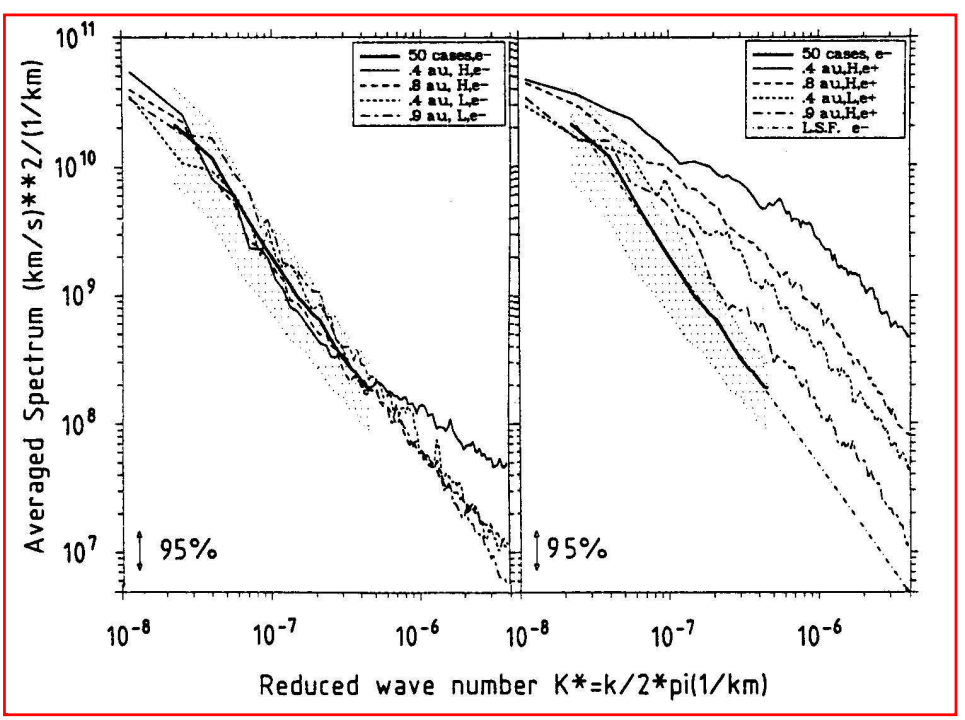

Figure 36: Power density spectra $e^{-}$and $e^{+}$computed from $\delta z^{-}$and $\delta z^{+}$fluctuations. Spectra have been computed within fast $(\mathrm{H})$ and slow $(\mathrm{L})$ streams around 0.4 and $0.9 \mathrm{AU}$ as indicated by different line styles. The thick line represents the average power spectrum obtained from all the about $50 e^{-}$spectra, regardless of distances and wind speed. The shaded area is the $1 \sigma$ width related to the average (adopted from Tu and Marsch, 1990b, (c) 1990 American Geophysical Union, reproduced by permission of American Geophysical Union).

The $e^{-}$spectrum, if we exclude the high frequency range of the spectrum relative to fast wind at $0.4 \mathrm{AU}$, shows an average power law profile with a slope of -1.64 , consistent with Kolmogorov's scaling. The lack of radial evolution of $e^{-}$spectrum brought Tu and Marsch (1990a) to name it "the background spectrum" of solar wind turbulence.

Quite different is the behavior of $e^{+}$spectrum. Close to the Sun and within fast wind, this spectrum appears to be flatter at low frequency and steeper at high frequency. The overall evolution is towards the "background spectrum" by the time the wind reaches $0.8 \mathrm{AU}$.

In particular, Figure 36 tells us that the radial evolution of the normalized cross-helicity has to be ascribed mainly to the radial evolution of $e^{+}$rather than to both Alfvénic fluctuations (Tu and Marsch, 1990a). In addition, Figure 37, relative to the Elsässer ratio $r_{\mathrm{E}}$, shows that the hourly frequency range, up to $\sim 2 \cdot 10^{-3} \mathrm{~Hz}$, is the most affected by this radial evolution.

As a matter of fact, this radial evolution can be inferred from Figure 38 where values of $e^{-}$and $e^{+}$together with solar wind speed, magnetic field intensity, and magnetic field and particle density compression are shown between 0.3 and $1 \mathrm{AU}$ during the primary mission of Helios 2 . It clearly appears that enhancements of $e^{-}$and depletion of $e^{+}$are connected to compressive events, particularly within slow wind. Within fast wind the average level of $e^{-}$is rather constant during the radial excursion while the level of $e^{+}$dramatically decreases with a consequent increase of the Elsässer ratio (see Appendix 13.3.1).

Further ecliptic observations (see Figure 39) do not indicate any clear radial trend for the Elsässer ratio between 1 and $5 \mathrm{AU}$, and its value seems to fluctuate between 0.2 and 0.4 .

However, low values of the normalized cross-helicity can also be associated with a particular type of uncompressive events, which Tu and Marsch (1991) called Magnetic Field Directional Turnings or MFDT. These events, found within slow wind, were characterized by very low values of $\sigma_{\mathrm{c}}$ close to zero and low values of the Alfvén ratio, around 0.2. Moreover, the spectral slope of $e^{+}, e^{-}$and 


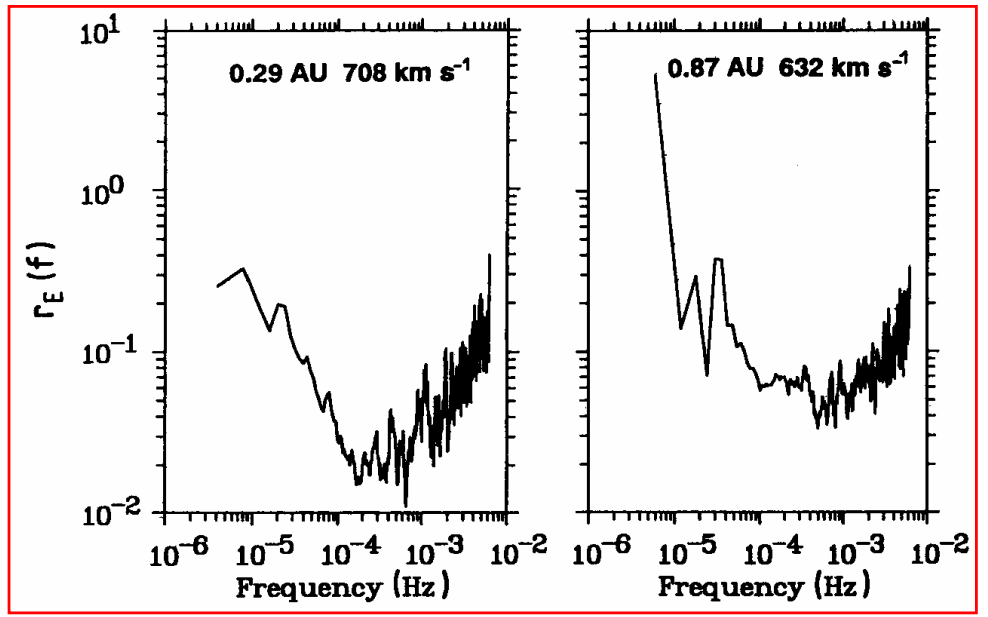

Figure 37: Ratio of $e^{-}$over $e^{+}$within fast wind at 0.3 and 0.9 AU in the left and right panels, respectively (adopted from Marsch and Tu, 1990a, (C) 1990 American Geophysical Union, reproduced by permission of American Geophysical Union).

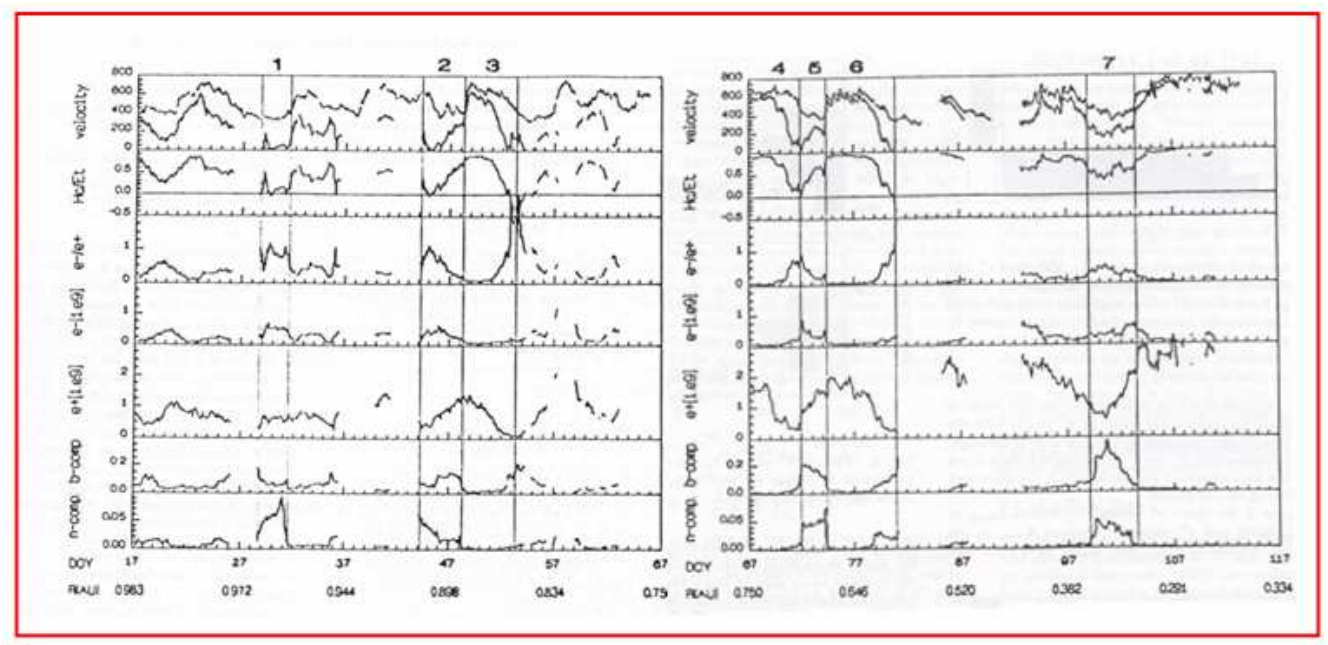

Figure 38: Upper panel: solar wind speed and solar wind speed multiplied by $\sigma_{\mathrm{c}}$. In the lower panels the authors reported: $\sigma_{\mathrm{c}}, r_{\mathrm{E}}, e^{-}, e^{+}$, magnetic compression, and number density compression, respectively (adopted from Bruno and Bavassano, 1991, (c) 1991 American Geophysical Union, reproduced by permission of American Geophysical Union).

Living Reviews in Solar Physics

http://www. livingreviews .org/lrsp-2005-4 


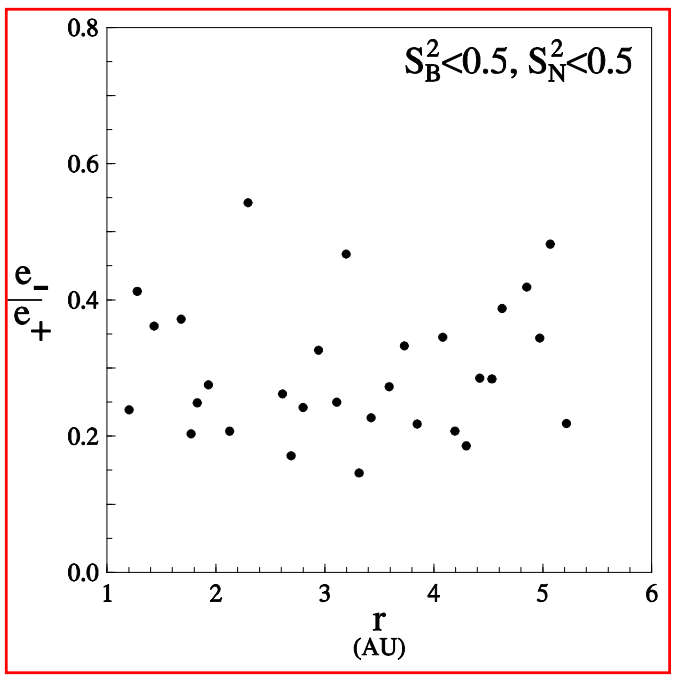

Figure 39: Ratio of $e^{-}$over $e^{+}$within fast wind between 1 and $5 \mathrm{AU}$ as observed by Ulysses in the ecliptic (adopted from Bavassano et al., 2001, (C) 2001 American Geophysical Union, reproduced by permission of American Geophysical Union).

the power associated with the magnetic field fluctuations was close to the Kolmogorov slope. These intervals were only scarcely compressive, and short period fluctuations, from a few minutes to about $40 \mathrm{~min}$, were nearly pressure balanced. Thus, differently from what had previously been observed by Bruno et al. (1989), who found low values of cross-helicity often accompanied by compressive events, these MFDTs were mainly uncompressive. In these structures most of the fluctuating energy resides in the magnetic field rather than velocity as shown in Figure 40 taken from Tu and Marsch (1991). It follows that the amplitudes of the fluctuating Alfvénic fields $\delta z^{ \pm}$result to be comparable and, consequently, the derived parameter $\sigma_{\mathrm{c}} \rightarrow 0$. Moreover, the presence of these structures would also be able to explain the fact that $r_{\mathrm{A}}<1$. Tu and Marsch (1991) suggested that these fluctuations might derive from a special kind of magnetic structures, which obey the MHD equations, for which $(\mathbf{B} \cdot \nabla) \mathbf{B}=0$, field magnitude, proton density, and temperature are all constant. The same authors suggested the possibility of an interplanetary turbulence mainly made of outwardly propagating Alfvén waves and convected structures represented by MFDTs. In other words, this model assumed that the spectrum of $e^{-}$would be caused by MFDTs. The different radial evolution of the power associated with these two kind of components would determine the radial evolution observed in both $\sigma_{\mathrm{c}}$ and $r_{\mathrm{A}}$. Although the results were not quantitatively satisfactory, they did show a qualitative agreement with the observations.

These convected structures are an important ingredient of the turbulent evolution of the fluctuations and can be identified as the 2D incompressible turbulence suggested by Matthaeus et al. (1990) and Tu and Marsch (1991).

\subsubsection{On the nature of Alfvénic fluctuations}

The Alfvénic nature of outward modes has been widely recognized through several frequency decades up to periods of the order of several hours in the s/c rest frame (Bruno et al., 1985). Conversely, the nature of those fluctuations identified by $\delta z^{-}$, called "inward Alfvén modes", is still not completely clear. There are many clues which would suggest that these fluctuations, especially in the hourly frequencies range, have a non-Alfvénic nature. Several studies on this topic 


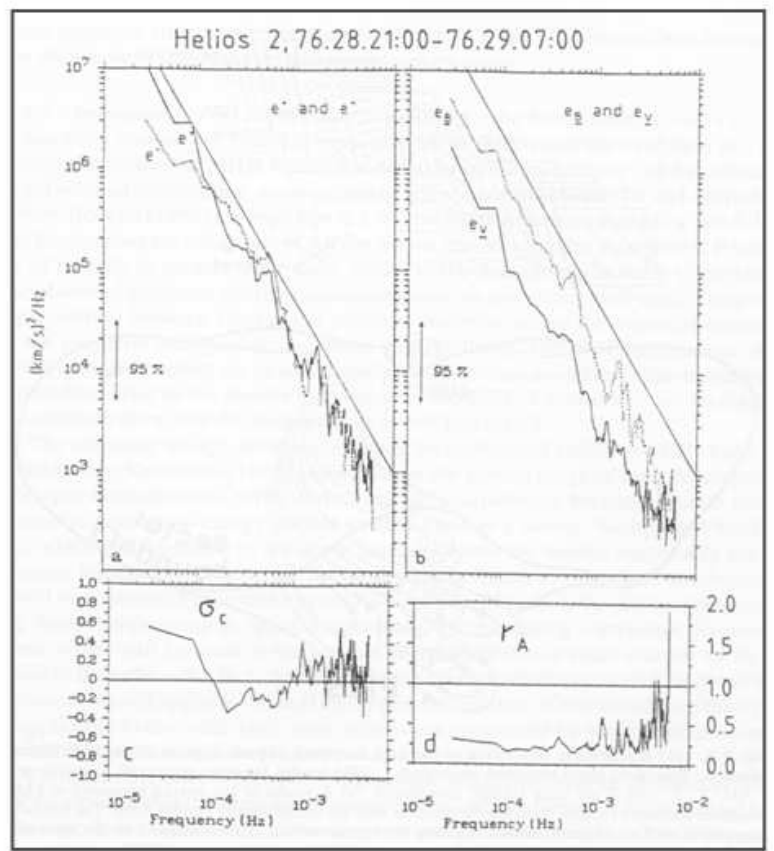

Figure 40: Left column: $e^{+}$and $e^{-}$spectra (top) and $\sigma_{\mathrm{c}}$ (bottom) during a slow wind interval at $0.9 \mathrm{AU}$. Right column: kinetic $e_{v}$ and magnetic $e_{B}$ energy spectra (top) computed from the trace of the relative spectral tensor, and spectrum of the Alfvén ratio $r_{\mathrm{A}}$ (bottom) (adopted from Tu and Marsch, 1991). 
in the low frequency range have suggested that structures convected by the wind could well mimic non-existent inward propagating modes (see the review by Tu and Marsch, 1995a). However, other studies (Tu et al., 1989b) have also found, in the high frequency range and within fast streams, a certain anisotropy in the components which resembles the same anisotropy found for outward modes. So, these observations would suggest a close link between inward modes at high frequency and outward modes, possibly the same nature.

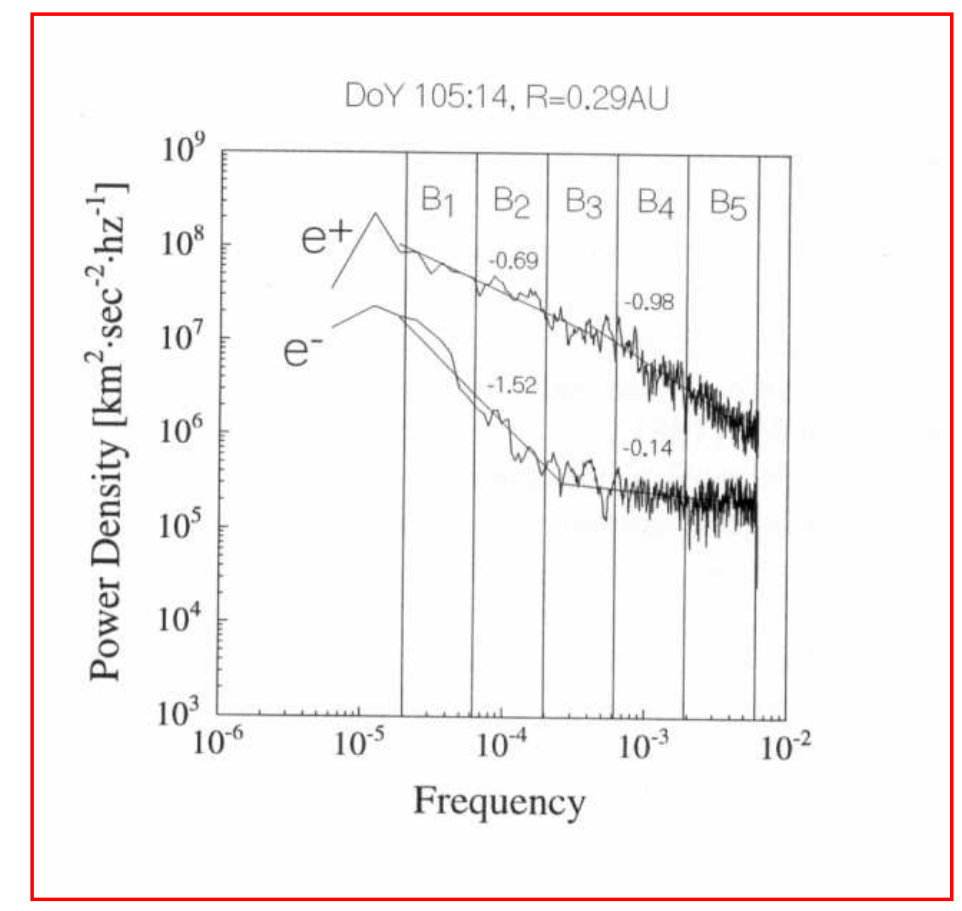

Figure 41: Power density spectra for $e^{+}$and $e^{-}$during a high velocity stream observed at $0.3 \mathrm{AU}$. Best fit lines for different frequency intervals and related spectral indices are also shown. Vertical lines fix the limits of five different frequency intervals analyzed by Bruno et al. (1996) (adopted from Bruno et al., 1996).

Figure 41 shows power density spectra for $e^{+}$and $e^{-}$during a high velocity stream observed at $0.3 \mathrm{AU}$ (similar spectra can be also found in the paper by Grappin et al., 1990 and Tu et al., 1989b). The observed spectral indices, reported on the plot, are typically found within high velocity streams encountered at short heliocentric distances. Bruno et al. (1996) analyzed the power relative to $e^{+}$and $e^{-}$modes, within five frequency bands, ranging from roughly $12 \mathrm{~h}$ to $3 \mathrm{~min}$, delimited by the vertical solid lines equally spaced in log-scale. The integrated power associated with $e^{+}$and $e^{-}$within the selected frequency bands is shown in Figure 42. Passing from slow to fast wind $e^{+}$grows much more within the highest frequency bands. Moreover, there is a good correlation between the profiles of $e^{-}$and $e^{+}$within the first two highest frequency bands, as already noticed by Grappin et al. (1990) who looked at the correlation between daily averages of $e^{-}$and $e^{+}$in several frequency bands, even widely separated in frequency. The above results stimulated these authors to conclude that it was reminiscent of the non-local coupling in $\mathbf{k}$-space between opposite modes found by Grappin et al. (1982) in homogeneous MHD. Expansion effects were also taken into account by Velli et al. (1990) who modeled inward modes as that fraction of outward modes back-scattered by the inhomogeneities of the medium due to expansion effects (Velli et al., 1989). 
However, following this model we would often expect the two populations to be somehow related to each other but, in situ observations do not favor this kind of forecast (Bavassano and Bruno, 1992)

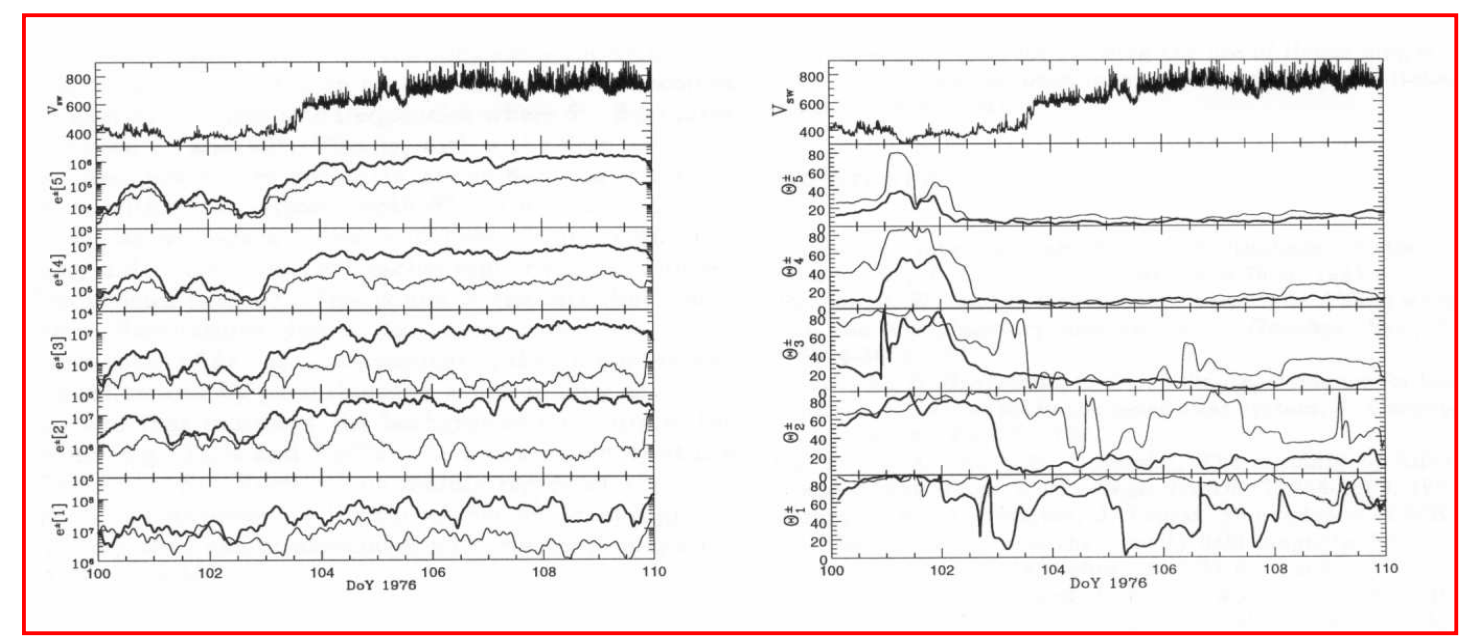

Figure 42: Left panel: wind speed profile is shown in the top panel. Power density associated with $e^{+}$(thick line) and $e^{-}$(thin line), within the 5 frequency bands chosen, is shown in the lower panels. Right panel: wind speed profile is shown in the top panel. Values of the angle between the minimum variance direction of $\delta z^{+}$(thick line) and $\delta z^{-}$(thin line) and the direction of the ambient magnetic field are shown in the lower panels, relatively to each frequency band (adopted from Bruno et al., 1996).

An alternative generation mechanism was proposed by Tu et al. (1989b) based on the parametric decay of $e^{+}$in high frequency range (Galeev and Oraevskii, 1963). This mechanism is such that large amplitude Alfvén waves, unstable to perturbations of random field intensity and density fluctuations, would decay into two secondary Alfvén modes propagating in opposite directions and a sound-like wave propagating in the same direction of the pump wave. Most of the energy of the mother wave would go into the sound-like fluctuation and the backward propagating Alfvén mode. On the other hand, the production of $e^{-}$modes by parametric instability is not particularly fast if the plasma $\beta \sim 1$, like in the case of solar wind (Goldstein, 1978; Derby, 1978), since this condition slows down the growth rate of the instability. It is also true that numerical simulations by Malara et al. (2000, 2001, 2002), and Primavera et al. (2003) have shown that parametric decay can still be thought as a possible mechanism of local production of turbulence within the polar wind (see Section 4). However, the strong correlation between $e^{+}$and $e^{-}$profiles found only within the highest frequency bands would support this mechanism and would suggest that $e^{-}$modes within these frequency bands would have an Alfvénic nature. Another feature shown in Figure 42 that favors these conclusions is the fact that both $\delta z^{+}$and $\delta z^{-}$keep the direction of their minimum variance axis aligned with the background magnetic field only within the fast wind, and exclusively within the highest frequency bands. This would not contradict the view suggested by Barnes (1981). Following this model, the majority of Alfvénic fluctuations propagating in one direction have the tip of the magnetic field vector randomly wandering on the surface of half a sphere of constant radius, and centered along the ambient field $\mathbf{B}_{\circ}$. In this situation the minimum variance would be oriented along $\mathbf{B}_{\circ}$, although this would not represent the propagation direction of each wave vector which could propagate even at large angles from this direction. This situation can be seen in the

Living Reviews in Solar Physics

http://www. livingreviews . org/lrsp-2005-4 
right hand panel of Figure 89 of Section 9, which refers to a typical Alfvénic interval within fast wind. Moreover, $\delta z^{+}$fluctuations show a persistent anisotropy throughout the fast stream since the minimum variance axis remains quite aligned to the background field direction. This situation downgrades only at the very low frequencies where $\theta^{+}$starts wandering between $0^{\circ}$ and $90^{\circ}$. On the contrary, in slow wind, since Alfvénic modes have a smaller amplitude, compressive structures due to the dynamic interaction between slow and fast wind or, of solar origin, push the minimum variance direction to larger angles with respect to $\mathbf{B}_{\circ}$, not depending on the frequency range.

In a way, we can say that within the stream, both $\theta^{+}$and $\theta^{-}$show a similar behavior as we look at lower and lower frequencies. The only difference is that $\theta^{-}$reaches higher values at higher frequencies than $\theta^{+}$. This was interpreted (Bruno et al., 1996) as due to the fact that transverse fluctuations of $\delta z^{-}$carry much less power than those of $\delta z^{+}$and, consequently, they are more easily influenced by perturbations represented by the background, convected structure of the wind (e.g., TD's and PBS's). As a consequence, at low frequency $\delta z^{-}$fluctuations may represent a signature of the compressive component of the turbulence while, at high frequency, they might reflect the presence of inward propagating Alfvén modes. Thus, while for periods of several hours $\delta z^{+}$fluctuations can still be considered as the product of Alfvén modes propagating outward (Bruno et al., 1985), $\delta z^{-}$fluctuations are rather due to the underlying convected structure of the wind. In other words, high frequency turbulence can be looked at mainly as a mixture of inward and outward Alfvénic fluctuations plus, presumably, sound-like perturbations (Marsch and $\mathrm{Tu}, 1993 \mathrm{a})$. On the other hand, low frequency turbulence would be made of outward Alfvénic fluctuations and static convected structures representing the inhomogeneities of the background medium. 


\section{Observations of MHD Turbulence in the Polar Wind}

In 1994-1995 Ulysses gave us the opportunity to look at the solar wind out-of-the-ecliptic, providing us with new exciting observations. For the first time heliospheric instruments were sampling pure, fast solar wind, free of any dynamical interaction with slow wind. There is one figure that within our scientific community has become as popular as "La Gioconda" by Leonardo da Vinci within the world of art. This figure produced at LANL (McComas et al., 1998) is shown in the upper left panel of Figure 43, which has been taken from a successive paper by (McComas et al., 2003), and summarizes the most important aspects of the large scale structure of the polar solar wind during the minimum of the solar activity phase, as indicated by the low value of the Wolf's number reported in the lower panel. It shows speed profile, proton number density profile and magnetic field polarity vs. heliographic latitude during the first complete Ulysses' polar orbit. Fast wind fills up north and south hemispheres of the Sun almost completely, except a narrow latitudinal belt around the equator, where the slow wind dominates. Flow velocity, which rapidly increases from the equator towards higher latitudes, quickly reaches a plateau and the wind escapes the polar regions with a rather uniform speed. Moreover, polar wind is characterized by a lower number density and shows rather uniform magnetic polarity of opposite sign, depending on the hemisphere. Thus, the main difference between ecliptic and polar wind is that this last one completely lacks of dynamical interactions with slower plasma and freely flows into the interplanetary space. The presence or not of this phenomenon, as we will see in the following pages, plays a major role in the development of MHD turbulence during the wind expansion.

During solar maximum (look at the upper right panel of Figure 43) the situation dramatically changes and the equatorial wind extends to higher latitudes, to the extent that there is no longer difference between polar and equatorial wind.

\subsection{Evolving turbulence in the polar wind}

Ulysses observations gave us the possibility to test whether or not we could forecast the turbulent evolution in the polar regions on the basis of what we had learned in the ecliptic. We knew that, in the ecliptic, velocity shear, parametric decay, and interaction of Alfvénic modes with convected structures (see Sections 3.2.1, 5.1) all play some role in the turbulent evolution and, before Ulysses reached the polar regions of the Sun, three possibilities were given:

i. Alfvénic turbulence would have not relaxed towards standard turbulence because the large scale velocity shears would have been much less relevant (Grappin et al., 1991);

ii. since the magnetic field would be smaller far from the ecliptic, at large heliocentric distances, even small shears would lead to an isotropization of the fluctuations and produce a turbulent cascade faster than the one observed at low latitudes, and the subsequent evolution would take less (Roberts et al., 1990);

iii. there would still be evolution due to interaction with convected plasma and field structures but it would be slower than in the ecliptic since the power associated with Alfvénic fluctuations would largely dominate over the inhomogeneities of the medium. Thus, Alfvénic correlations should last longer than in the ecliptic plane, with a consequent slower evolution of the normalized cross-helicity (Bruno, 1992).

A fourth possibility was added by Tu and Marsch (1995a), based on their model ( $\mathrm{Tu}$ and Marsch, 1993). Following this model they assumed that polar fluctuations were composed by outward Alfvénic fluctuations and MFDT. The spectra of these components would decrease with radial distance because of a WKB evolution and convective effects of the diverging flow. As the distance increases, the field becomes more transverse with respect to the radial direction, the s/c

Living Reviews in Solar Physics

http: //www . livingreviews . org/lrsp-2005-4 


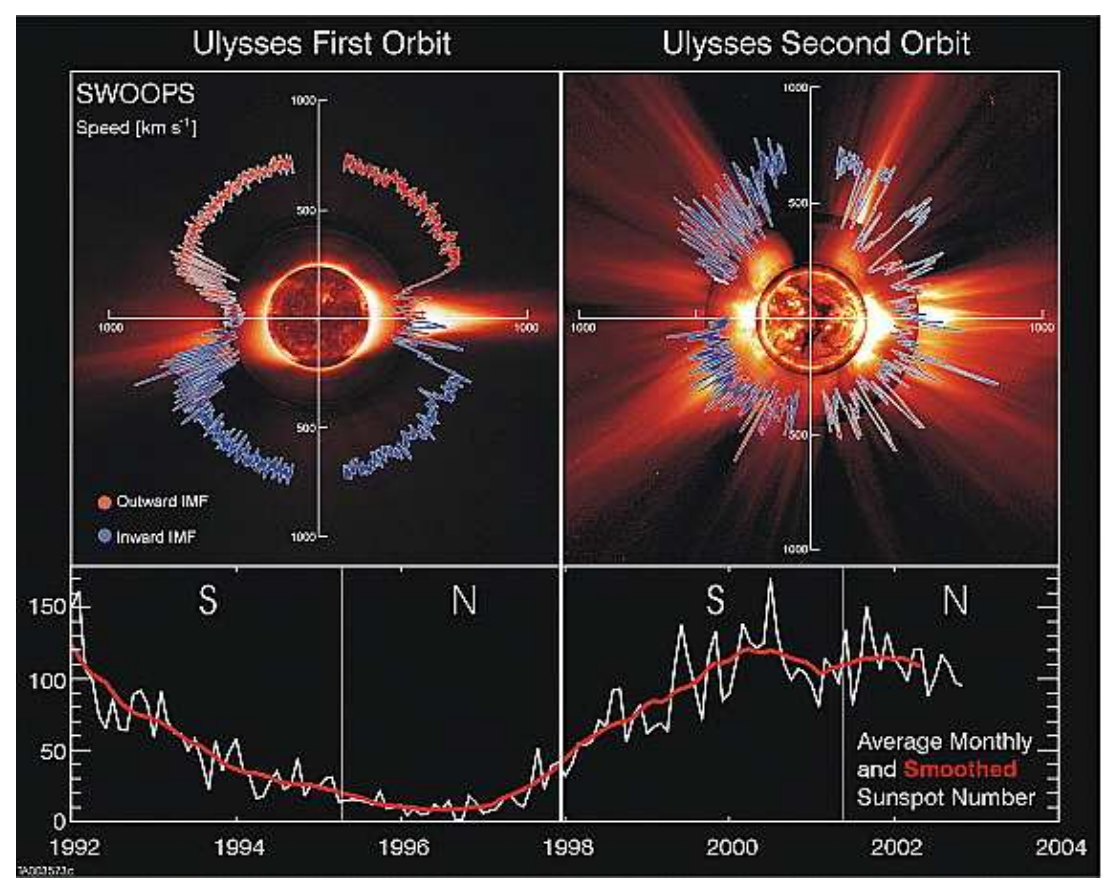

Figure 43: Large scale solar wind profile as a function of latitude during minimum (left panel) and maximum (right panel) solar cycle phases. The sunspot number is also shown at the bottom panels (adopted from McComas et al., 2003, (c) 2003 American Geophysical Union, reproduced by permission of American Geophysical Union). 
would sample more convective structures and, as a consequence, would observe a decrease of both $\sigma_{\mathrm{c}}$ and $r_{\mathrm{A}}$.

Today we know that polar Alfvénic turbulence evolves in the same way it does in the ecliptic plane, but much more slowly. Moreover, the absence of strong velocity shears and enhanced compressive phenomena suggests that also some other mechanism based on parametric decay instability might play some role in the local production of turbulence (Bavassano et al., 2000a; Malara et al., 2001, 2002; Primavera et al., 2003).

The first results of Ulysses magnetic field and plasma measurements in the polar regions, i.e., above $\pm 30^{\circ}$ latitude (left panel of Figure 43), revealed the presence of Alfvénic correlations in a frequency range from less than 1 to more than $10 \mathrm{~h}$ (Balogh et al., 1995; Smith et al., 1995; Goldstein et al., 1995a) in very good agreement with ecliptic observations (Bruno et al., 1985). However, it is worth noticing that Helios observations referred to very short heliocentric distances around $0.3 \mathrm{AU}$ while the above Ulysses observations were taken up to $4 \mathrm{AU}$. As a matter of fact, these long period Alfvén waves observed in the ecliptic, in the inner solar wind, become less prominent as the wind expands due to stream-stream dynamical interaction effects (Bruno et al., 1985) and strong velocity shears (Roberts et al., 1987a). At high latitude, the relative absence of enhanced dynamical interaction between flows at different speed and, as a consequence, the absence of strong velocity shears favors the survival of these extremely low frequency Alfvénic fluctuations for larger heliocentric excursions.

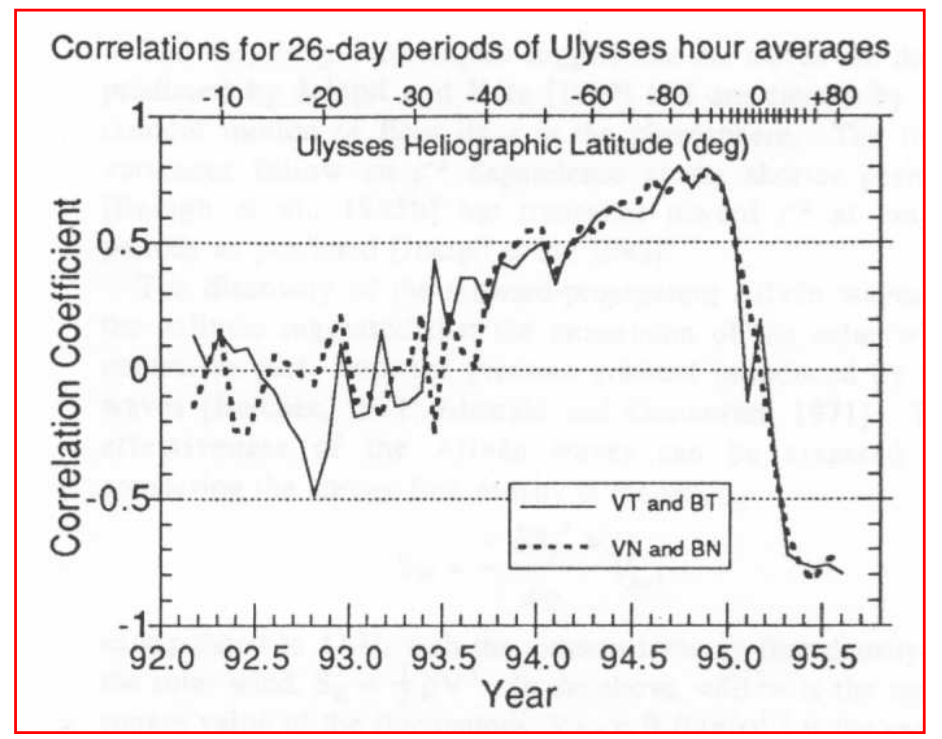

Figure 44: Magnetic field and velocity hourly correlation vs. heliographic latitude (adopted from Smith et al., 1995, (c) 1995American Geophysical Union, reproduced by permission of American Geophysical Union).

Figure 44 shows the hourly correlation coefficient for the transverse components of magnetic and velocity fields as Ulysses climbs to the south pole and during the fast latitude scanning that brought the s/c from the south to the north pole of the Sun in just half a year. While the equatorial phase of Ulysses journey is characterized by low values of the correlation coefficients, a gradual increase can be noticed starting at half of year 1993 when the s/c starts to increase its heliographic latitude from the ecliptic plane up to $80.2^{\circ}$ south, at the end of 1994 . Not only the degree of $\delta \mathbf{b}-\delta \mathbf{v}$ correlation resembled Helios observations but also the spectra of these

Living Reviews in Solar Physics

http: //www. livingreviews . org/lrsp-2005-4 
fluctuations showed characteristics which were very similar to those observed in the ecliptic within fast wind like the spectral index of the components, that was found to be flat at low frequency and more Kolmogorov-like at higher frequencies (Smith et al., 1995). Balogh et al. (1995) and Forsyth et al. (1996) discussed magnetic fluctuations in terms of latitudinal and radial dependence of their variances. Similarly to what had been found within fast wind in the ecliptic (Mariani et al., 1978; Bavassano et al., 1982b; Tu et al., 1989b; Roberts et al., 1992), variance of magnetic magnitude was much less than the variance associated with the components. Moreover, transverse variances had consistently higher values than the one along the radial direction and were also much more sensitive to latitude excursion, as shown in Figure 45. In addition, the level of the normalized

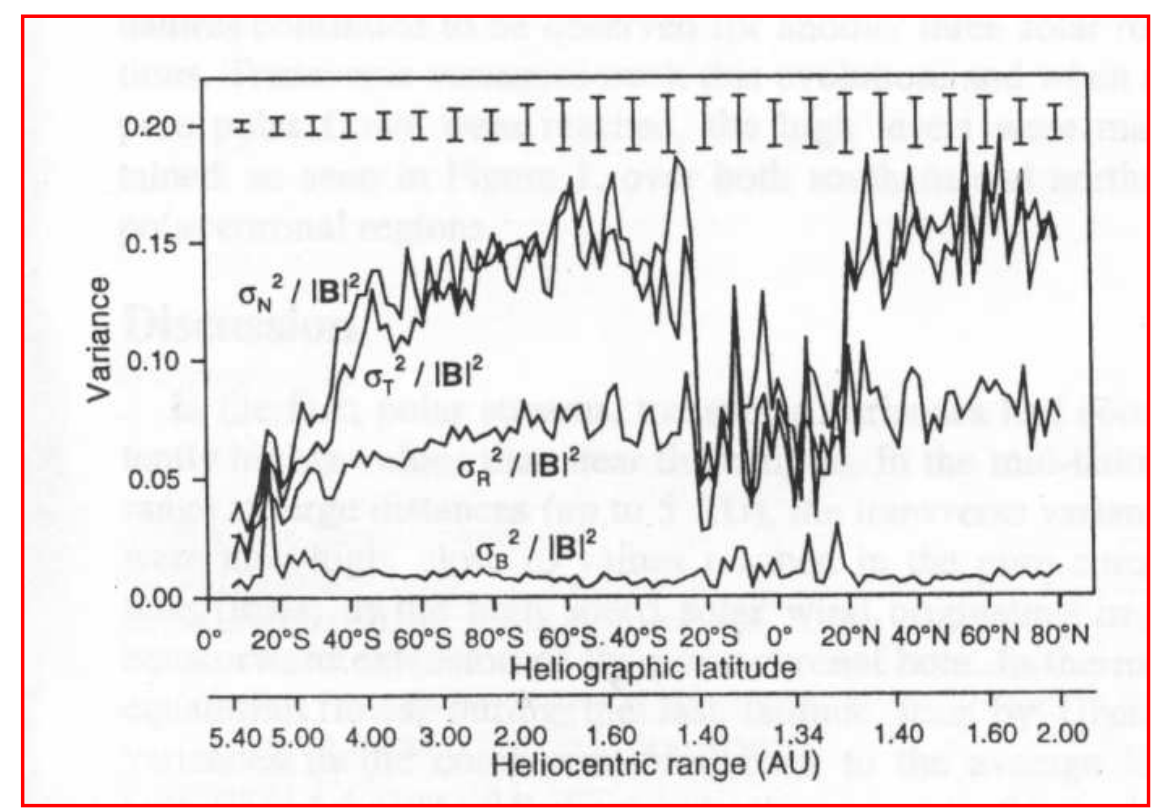

Figure 45: Normalized magnetic field components and magnitude hourly variances plotted vs. heliographic latitude during a complete latitude survey by Ulysses (adopted from Forsyth et al., 1996, (c) 1996 American Geophysical Union, reproduced by permission of American Geophysical Union).

hourly variances of the transverse components observed during the ecliptic phase, right after the compressive region ahead of corotating interacting regions, was maintained at the same level once the s/c entered the pure polar wind. Again, these observations showed that the fast wind observed in the ecliptic was coming from the equatorward extension of polar coronal holes.

Horbury et al. (1995c) and Forsyth et al. (1996) showed that the interplanetary magnetic field fluctuations observed by Ulysses continuously evolve within the fast polar wind, at least out to 4 AU. Since this evolution was observed within the polar wind, rather free of co-rotating and transient events like those characterizing low latitudes, they concluded that some other mechanism was at work and this evolution was an intrinsic property of turbulence.

Results in Figure 46 show the evolution of three different time scales. The smallest time scales show a clear evolution that keeps on going past the highest latitude on day 256, strongly suggesting that this evolution is radial rather than latitudinal effect. Horbury et al. (1996a) worked on determining the rate of turbulent evolution for the polar wind. They calculated the spectral index at different frequencies from the scaling of the second order structure function (see Section 7 


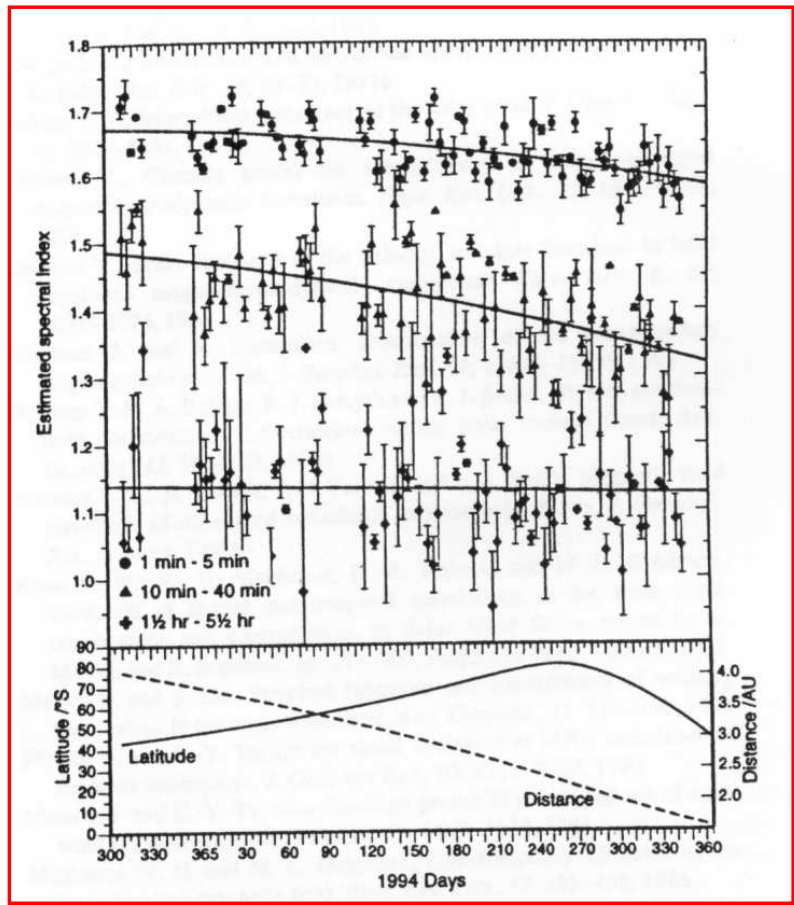

Figure 46: Spectral indexes of magnetic fluctuations within three different time scales as indicated in the plot. The bottom panel shows heliographic latitude and heliocentric distance of Ulysses (adopted from Horbury et al., 1995c, (c) 1995 American Geophysical Union, reproduced by permission of American Geophysical Union). 
and papers by Burlaga (1992a), Burlaga (1992b), Marsch and Tu (1993a), Ruzmaikin et al. (1995), and Horbury et al. (1996b)) since the spectral scaling $\alpha$ is related to the scaling of the structure function $s$ by the following relation: $\alpha=s+1$ (Monin and Yaglom, 1975). Horbury et al. (1996a), studying variations of the spectral index with frequency for polar turbulence, found that there are two frequency ranges where the spectral index is rather steady. The first range is around $10^{-2} \mathrm{~Hz}$ with a spectral index around $-5 / 3$, while the second range is at very low frequencies with a spectral index around -1 . This last range is the one where Goldstein et al. (1995a) found the best example of Alfvénic fluctuations. Similarly, ecliptic studies found that the best Alfvénic correlations belonged to the hourly, low frequency regime (Bruno et al., 1985).

Horbury et al. (1995a) presented an analysis of the high latitude magnetic field using a fractal method. Within the solar wind context, this method has been described for the first time by Burlaga and Klein (1986) and Ruzmaikin et al. (1993), and is based on the estimate of the scaling of the length function $L(\tau)$ with the scale $\tau$. This function is closely related to the first order structure function and, if statistical self-similar, has scaling properties $L(\tau) \sim \tau^{\ell}$, where $\ell$ is the scaling exponent. It follows that $L(\tau)$ is an estimate of the amplitude of the fluctuations at scale $\tau$, and the relation that binds $L(\tau)$ to the variance of the fluctuations $(\delta B)^{2} \sim \tau^{s(2)}$ is:

$$
L(\tau) \sim N(\tau)\left[(\delta B)^{2}\right]^{1 / 2} \propto \tau^{s(2) / 2-1},
$$

where $N(\tau)$ represents the number of points at scale $\tau$ and scales like $\tau^{-1}$. Since the power density spectrum $W(f)$ is related to $(\delta B)^{2}$ through the relation $f W(f) \sim(\delta B)^{2}$, if $W(f) \sim f^{-\alpha}$, then $s(2)=\alpha-1$, and, as a consequence $\alpha=2 \ell+3$ (Marsch and Tu, 1996). Thus, it results very easy to estimate the spectral index at a given scale or frequency, without using spectral methods but simply computing the Length Function.

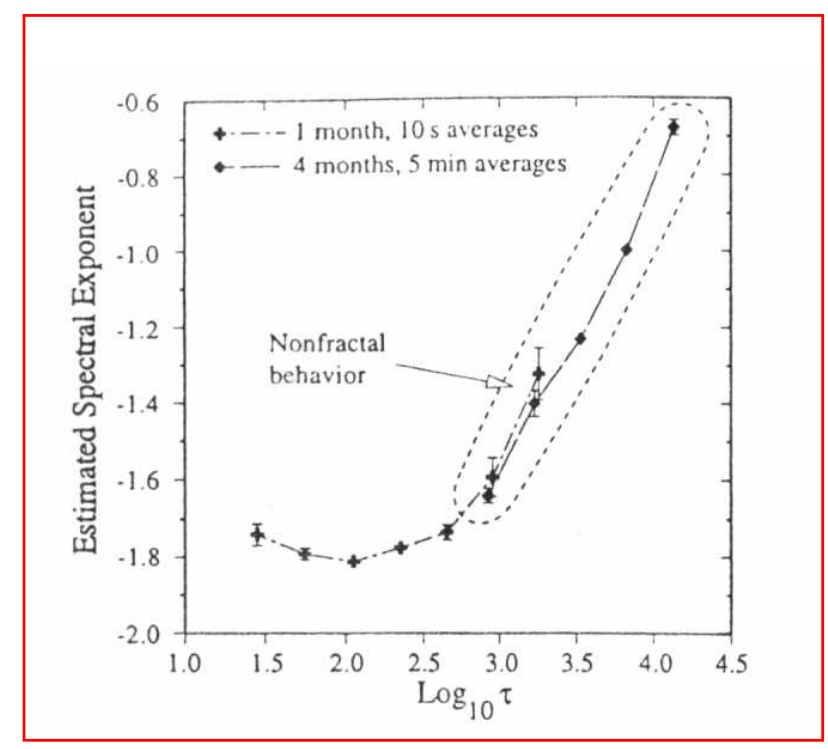

Figure 47: Spectral exponents for the $B_{z}$ component estimated from the length function computed from Ulysses magnetic field data, when the $\mathrm{s} / \mathrm{c}$ was at about $4 \mathrm{AU}$ and $\sim-50^{\circ}$ latitude. Different symbols refer to different time intervals as reported in the graph (figure adopted from Horbury et al., 1995a).

Results in Figure 47 show the existence of two different regimes, one with a spectral index around the Kolmogorov scaling extending from $10^{1.5}$ to $10^{3} \mathrm{~s}$ and, separated by a clear break- 
point at scales of $10^{3} \mathrm{~s}$, a flatter and flatter spectral exponent for larger and larger scales. These observations were quite similar to what had been observed by Helios 2 in the ecliptic, although the turbulence state recorded by Ulysses resulted to be more evolved than the situation seen at $0.3 \mathrm{AU}$ and, perhaps, more similar to the turbulence state observed around $1 \mathrm{AU}$, as shown by Marsch and Tu (1996). These authors compared the spectral exponents, estimated using the same method of Horbury et al. (1995a), from Helios 2 magnetic field observations at two different heliocentric distances: 0.3 and 1.0 AU. The comparison with Ulysses results is shown in Figure 48 where it appears rather clear that the slope of the $B_{z}$ spectrum experiences a remarkable evolution during the wind expansion between 0.3 and 4 AU. Obviously, this comparison is meaningful in the reasonable hypothesis that fluctuations observed by Helios 2 at $0.3 \mathrm{AU}$ are representative of outof-the-ecliptic solar wind (Marsch and Tu, 1996). This figure also shows that the degree of spectral evolution experienced by the fluctuations when observed at $4 \mathrm{AU}$ at high latitude, is comparable to Helios observations at $1 \mathrm{AU}$ in the ecliptic. Thus, the spectral evolution at high latitude is present although quite slower with respect to the ecliptic.

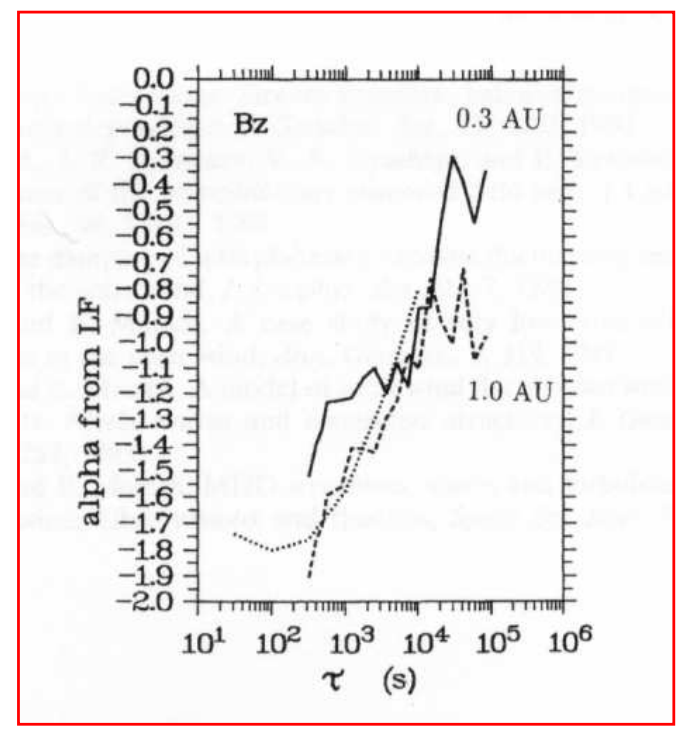

Figure 48: Spectral exponents for the $B_{z}$ component estimated from the length function computed from Helios and Ulysses magnetic field data. Ulysses length function (dotted line) is the same shown in the paper by Horbury et al. (1995a) when the s/c was at about $4 \mathrm{AU}$ and $\sim-50^{\circ}$ latitude (adopted from Marsch and Tu, 1996, (c) 1996 American Geophysical Union, reproduced by permission of American Geophysical Union).

Forsyth et al. (1996) studied the radial dependence of the normalized hourly variances of the components $B_{R}, B_{T}$ and $B_{N}$ and the magnitude $|\mathbf{B}|$ of the magnetic field (see Appendix 15 to learn about the $R T N$ reference system). The variance along the radial direction was computed as $\sigma_{R}{ }^{2}=<B_{R}^{2}>-<B_{R}>^{2}$ and successively normalized to $|\mathbf{B}|^{2}$ to remove the field strength dependence. Moreover, variances along the other two directions $T$ and $N$ were similarly defined. Fitting the radial dependence with a power law of the form $r^{-\alpha}$, but limiting the fit to the radial excursion between 1.5 and $3 \mathrm{AU}$, these authors obtained $\alpha=3.39 \pm 0.07$ for $\sigma_{\mathrm{r}}^{2}, \alpha=3.45 \pm 0.09$ for $\sigma_{T}^{2}, \alpha=3.37 \pm 0.09$ for $\sigma_{N}^{2}$, and $\alpha=2.48 \pm 0.14$ for $\sigma_{B}^{2}$. Thus, for hourly variances, the power associated with the components showed a radial dependence stronger than the one predicted by the WKB approximation, which would provide $\alpha=3$. These authors also showed that including data

Living Reviews in Solar Physics

http://www . livingreviews . org/Irsp-2005-4 
between 3 and $4 \mathrm{AU}$, corresponding to intervals characterized by compressional features mainly due to high latitude CMEs, they would obtain less steep radial gradients, much closer to a WKB type. These results suggested that compressive effects can feed energy at the smallest scales, counteracting dissipative phenomena and mimicking a WKB-like behavior of the fluctuations. However, they concluded that for lower frequencies, below the frequency break point, fluctuations do follow the WKB radial evolution.

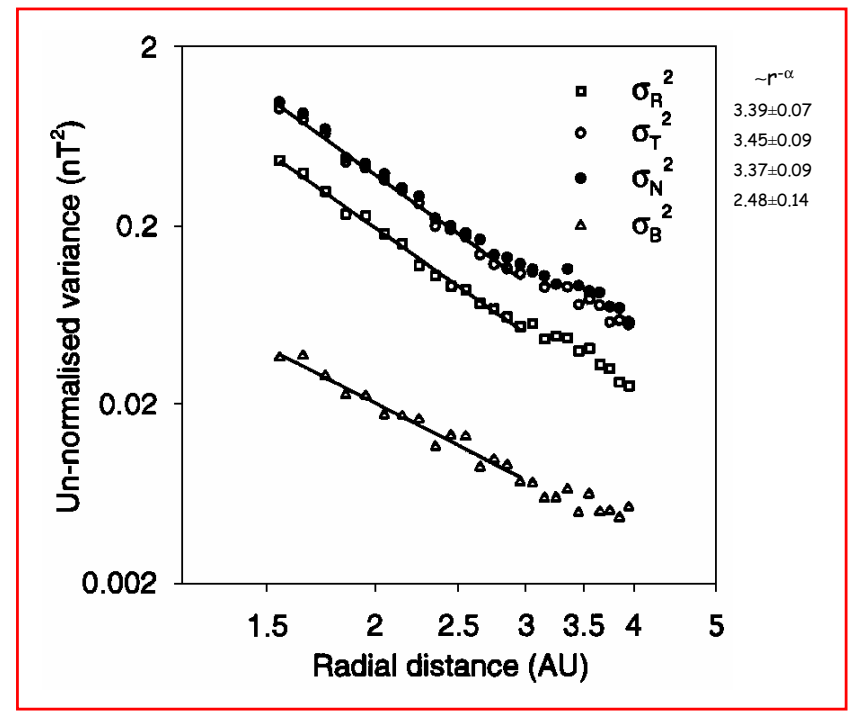

Figure 49: Hourly variances of the components and the magnitude of the magnetic field vs. radial distance from the Sun. The meaning of the different symbols is also indicated in the upper right corner (adopted from Forsyth et al., 1996, (C) 1996 American Geophysical Union, reproduced by permission of American Geophysical Union).

Horbury and Balogh (2001) presented a detailed comparison between Ulysses and Helios observations about the evolution of magnetic field fluctuations in high-speed solar wind. Ulysses results, between 1.4 and $4.1 \mathrm{AU}$, were presented as wave number dependence of radial and latitudinal power scaling. The first results of this analysis showed (Figure 3 of their work) a general decrease of the power levels with solar distance, in both magnetic field components and magnitude fluctuations. In addition, the power associated with the radial component was always less than that of the transverse components, as already found by Forsyth et al. (1996). However, Horbury and Balogh (2001), supposing a possible latitude dependence, performed a multiple linear regression of the type:

$$
\log _{10} w=A_{p}+B_{p} \log _{10} r+C_{p} \sin \theta,
$$

where $w$ is the power density integrated in a given spectral band, $r$ is the radial distance and $\theta$ is the heliolatitude $\left(0^{\circ}\right.$ at the equator). Moreover, the same procedure was applied to spectral index estimates $\alpha$ of the form $\alpha=A_{\alpha}+B_{\alpha} \log _{10} r+C_{\alpha} \sin \theta$. Results obtained for $B_{p}, C_{p}, B_{\alpha}, C_{\alpha}$ are shown in Figure 50.

On the basis of variations of spectral index and radial and latitudinal dependencies, these authors were able to identify four wave number ranges as indicated by the circled numbers in the top panel of Figure 50. Range 1 was characterized by a radial power decrease weaker than WKB (-3), positive latitudinal trend for components (more power at higher latitude) and negative for magnitude (less compressive events at higher latitudes). Range 2 showed a more rapid radial 


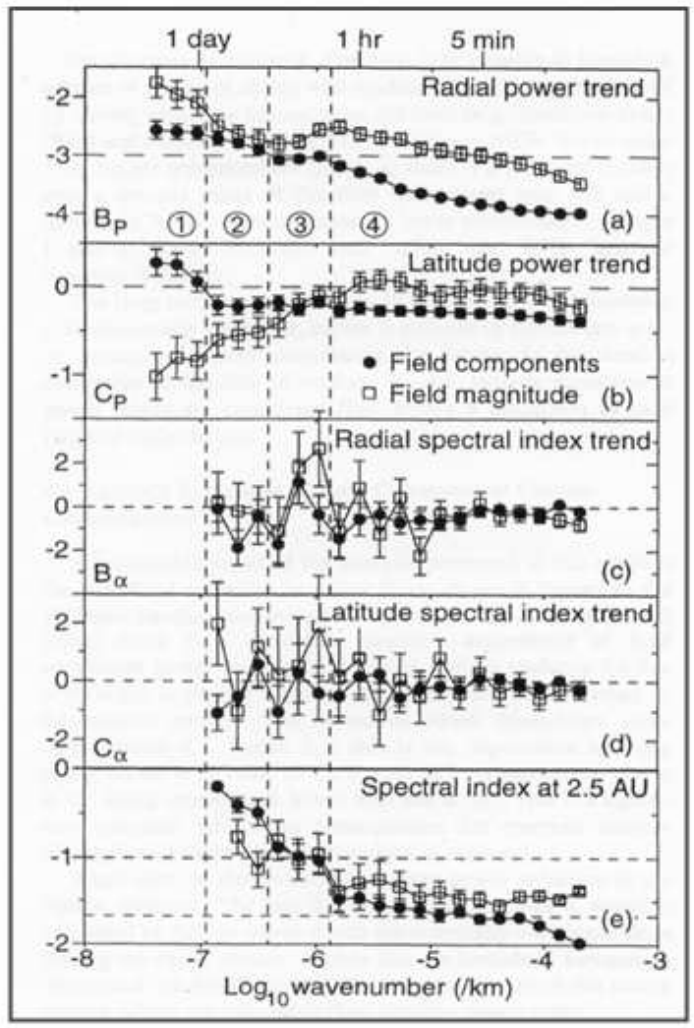

Figure 50: (a) Scale dependence of radial power, (b) latitudinal power, (c) radial spectral index, (d) latitudinal spectral index, and (e) spectral index computed at 2.5 AU. Solid circles refer to the trace of the spectral matrix of the components, open squares refer to field magnitude. Correspondence between wave number scale and time scale is based on a wind velocity of $750 \mathrm{~km} \mathrm{~s}^{-1}$ (adopted from Horbury and Balogh, 2001, (c) 2001 American Geophysical Union, reproduced by permission of American Geophysical Union). 
decrease of power for both magnitude and components and a negative latitudinal power trend, which implies less power at higher latitudes. Moreover, the spectral index of the components (bottom panel) is around 0.5 and tends to 0 at larger scales. Within range 3 the power of the components follows a WKB radial trend and the spectral index is around -1 for both magnitude and components. This hourly range has been identified as the most Alfvénic at low latitudes and its radial evolution has been recognized to be consistent with WKB radial index (Roberts, 1989; Marsch and Tu, 1990a). Even within this range, and also within the next one, the latitude power trend is slightly negative for both components and magnitude. Finally, range 4 is clearly indicative of turbulent cascade with a radial power trend of the components much faster than WKB expectation and becoming even stronger at higher wave numbers. Moreover, the radial spectral index reveals that steepening is at work only for the previous wave number ranges as expected since the breakpoint moves to smaller wave number during spectrum evolution. The spectral index of the components tends to $-5 / 3$ with increasing wave number while that of the magnitude is constantly flatter. The same authors gave an estimate of the radial scale-shift of the breakpoint during the wind expansion around $k \propto r^{1.1}$, in agreement with earlier estimates (Horbury et al., 1996a).

Although most of these results support previous conclusions obtained for the ecliptic turbulence, the negative value of the latitudinal power trend that starts within the second range, is totally unexpected. Horbury and Balogh (2001) and Horbury and Tsurutani (2001) estimated that the power observed at $80^{\circ}$ is about $30 \%$ less than that observed at $30^{\circ}$. These authors proposed a possible over-expansion of the polar coronal hole at higher latitudes. In addition, within the fourth range, field magnitude fluctuations radially decrease less rapidly than the fluctuations of the components, but do not show significant latitudinal variations. Finally, the smaller spectral index reveals that the high frequency range of the field magnitude spectrum shows a flattening.

The same authors investigated the anisotropy of these fluctuations as a function of radial and latitudinal excursion. Their results, reported in Figure 51, show that, at $2.5 \mathrm{AU}$, the lowest compressibility is recorded within the hourly frequency band (third and part of the fourth band), which has been recognized as the most Alfvénic frequency range. The anisotropy of the components confirms that the power associated with the transverse components is larger than that associated with the radial one, and this difference slightly tends to decrease at higher wave numbers.

As already shown by Horbury et al. (1995b), around the 5 min range, magnetic field fluctuations are transverse to the mean field direction the majority of the time. The minimum variance direction lies mainly within an angle of about $26^{\circ}$ from the average background field direction and fluctuations are highly anisotropic, such that the ratio between perpendicular to parallel power is about 30. Since during the observations reported in Horbury and Balogh (2001) and Horbury and Tsurutani (2001) the mean field resulted to be radially oriented most of the time, the radial minimum variance direction at short time scales is an effect induced by larger scales behavior.

Anyhow, radial and latitudinal anisotropy trends tend to disappear for higher frequencies. In the mean time, interesting enough, there is a strong radial increase of magnetic field compression (top panel of Figure 51), defined as the ratio between the power density associated with magnetic field intensity fluctuations and that associated with the fluctuations of the three components (Bavassano et al., 1982a; Bruno and Bavassano, 1991). The attempt to attribute this phenomenon to parametric decay of large amplitude Alfvén waves or dynamical interactions between adjacent flux tubes or interstellar pick-up ions was not satisfactory in all cases.

Comparing high latitude with low latitude results for high speed streams, Horbury and Balogh (2001) found remarkable good agreement between observations by Ulysses at $2.5 \mathrm{AU}$ and by Helios at $0.7 \mathrm{AU}$. In particular, Figure 52 shows Ulysses and Helios 1 spectra projected to $1 \mathrm{AU}$ for comparison.

It is interesting to notice that the spectral slope of the spectrum of the components for Helios 1 is slightly higher than that of Ulysses, suggesting a slower radial evolution of turbulence in the polar wind (Bruno, 1992; Bruno and Bavassano, 1992). However, the faster spectral evolution at 


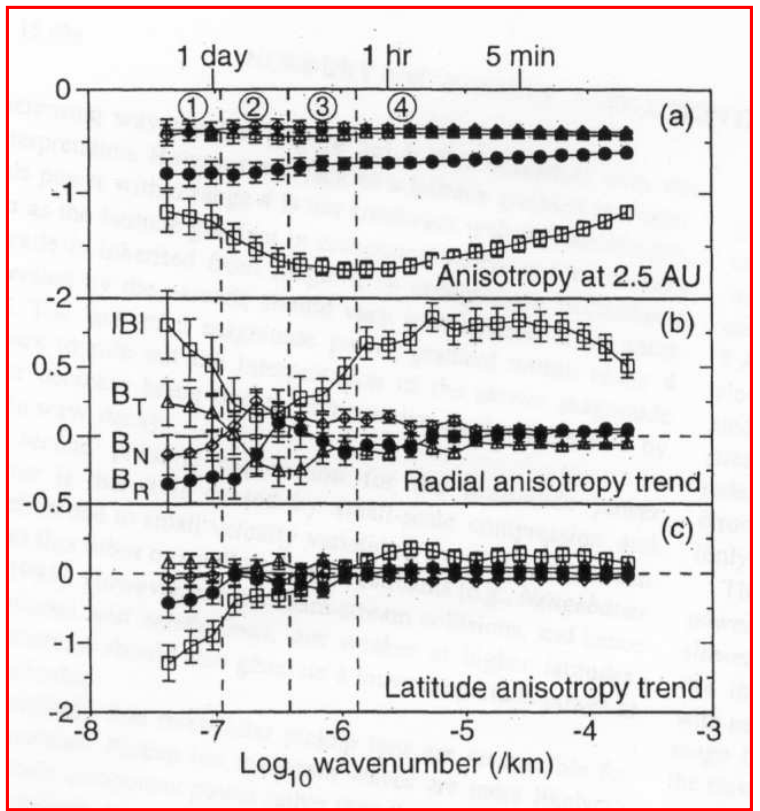

Figure 51: (a) Scale dependence of power anisotropy at 2.5 AU plotted as the $\log _{10}$ of the ratio of $B_{R}$ (solid circles), $B_{T}$ (triangles), $B_{N}$ (diamonds), and $|\mathbf{B}|$ (squares) to the trace of the spectral matrix; (b) the radial, and (c) latitudinal behavior of the same values, respectively (adopted from Horbury and Balogh, 2001, (c) 2001 American Geophysical Union, reproduced by permission of American Geophysical Union). 


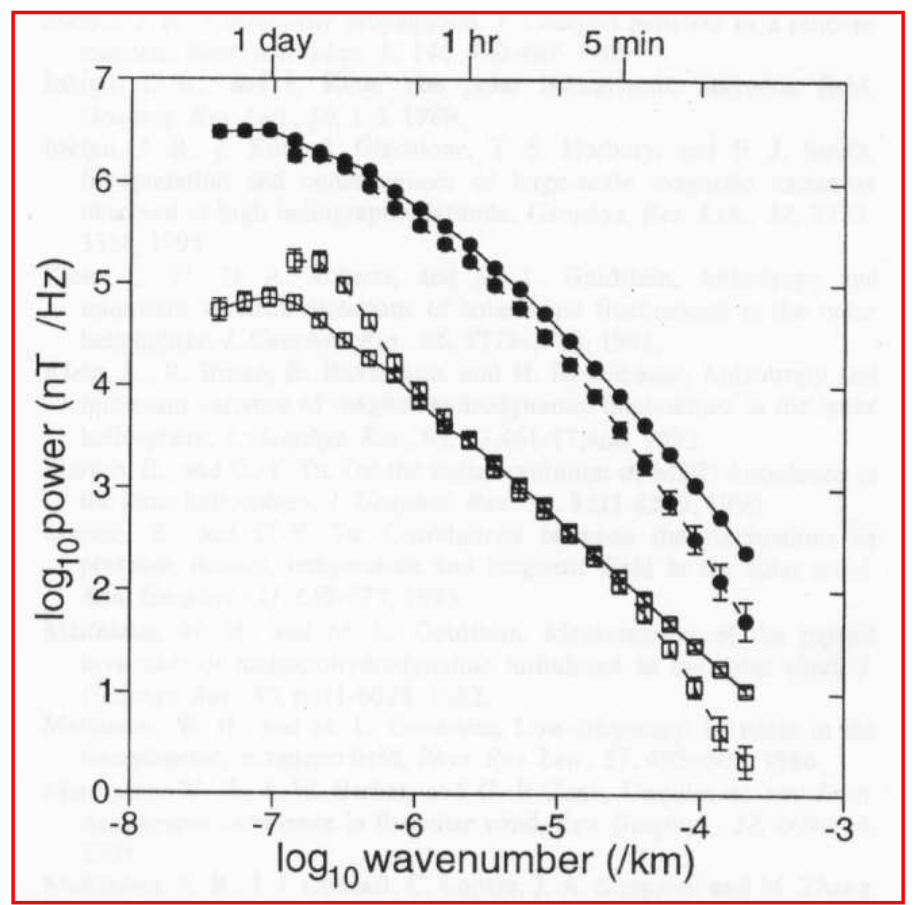

Figure 52: Power spectra of magnetic field components (solid circles) and magnitude (open squares) from Ulysses (solid line) and Helios 1 (dashed line). Spectra have been extrapolated to 1 AU using radial trends in power scalings estimated from Ulysses between 1.4 and 4.1 AU and Helios between 0.3 and 1 AU (adopted from Horbury and Balogh, 2001, (c) 2001 American Geophysical Union, reproduced by permission of American Geophysical Union). 
low latitudes does not lead to strong differences between the spectra.

\subsection{Polar turbulence studied via Elsässer variables}

Goldstein et al. (1995a) for the first time showed a spectral analysis of Ulysses observations based on Elsässer variables during two different time intervals, at $4 \mathrm{AU}$ and close to $-40^{\circ}$, and at $2 \mathrm{AU}$ and around the maximum southern pass, as shown in Figure 53. Comparing the two Ulysses observations it clearly appears that the spectrum closer to the Sun is less evolved than the spectrum measured farther out, as will be confirmed by the next Figure 54, where these authors reported the normalized cross-helicity and the Alfvén ratio for the two intervals. Moreover, following these authors, the comparison between Helios spectra at $0.3 \mathrm{AU}$ and Ulysses at 2 and 4 AU suggests that the radial scaling of $e^{+}$at the low frequency end of the spectrum follows the WKB prediction of $1 / r$ decrease (Heinemann and Olbert, 1980). However, the selected time interval for Helios s/c was characterized by rather slow wind taken during the rising phase the solar cycle, two conditions which greatly differ from those referring to Ulysses data. As a consequence, comparing Helios results with Ulysses results obtained within the fast polar wind might be misleading. It would be better to choose Helios observations within high speed corotating streams which resemble much better solar wind conditions at high latitude.

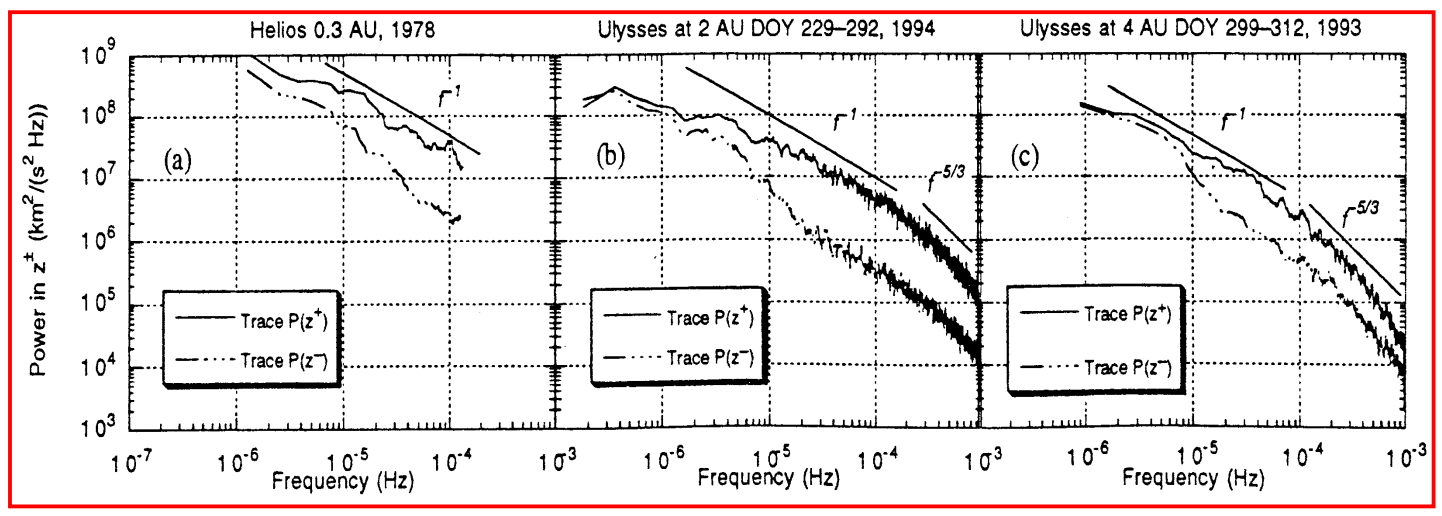

Figure 53: Trace of $e^{+}$(solid line) and $e^{-}$(dash-dotted line) power spectra. The central and right panels refer to Ulysses observations at 2 and $4 \mathrm{AU}$, respectively, when Ulysses was embedded in the fast southern polar wind during 1993-1994. The leftmost panel refers to Helios observations during 1978 at 0.3 AU (adopted from Goldstein et al., 1995a, (c) 1995 American Geophysical Union, reproduced by permission of American Geophysical Union).

Anyhow, results relative to the normalized cross-helicity $\sigma_{\mathrm{c}}$ (see Figure 54) clearly show high values of $\sigma_{\mathrm{c}}$, around 0.8 , which normally we observe in the ecliptic at much shorter heliocentric distances (Tu and Marsch, 1995a). A possible radial effect would be responsible for the depleted level of $\sigma_{\mathrm{c}}$ at $4 \mathrm{AU}$. Moreover, a strong anisotropy can also be seen for frequencies between $10^{-6}-10^{-5} \mathrm{~Hz}$ with the transverse $\sigma_{\mathrm{c}}$ much larger than the radial one. This anisotropy is somewhat lost during the expansion to $4 \mathrm{AU}$.

The Alfvén ratio (bottom panels of Figure 54) has values around 0.5 for frequencies higher than roughly $10^{-5} \mathrm{~Hz}$, with no much evolution between 2 and $4 \mathrm{AU}$. A result similar to what was for the first time obtained by Bruno et al. (1985), Marsch and Tu (1990a), and Roberts et al. (1990) in the ecliptic at about $1 \mathrm{AU}$. The low frequency extension of $r_{\mathrm{A} \perp}$ together with $\sigma_{\mathrm{c} \perp}$ is probably due to the fact that Ulysses was longitudinally sampling Alfvénic fluctuations and has been considered by these authors not really indicative of the existence of such low frequency Alfvénic fluctuations.

Living Reviews in Solar Physics

http: //www. livingreviews . org/lrsp-2005-4 


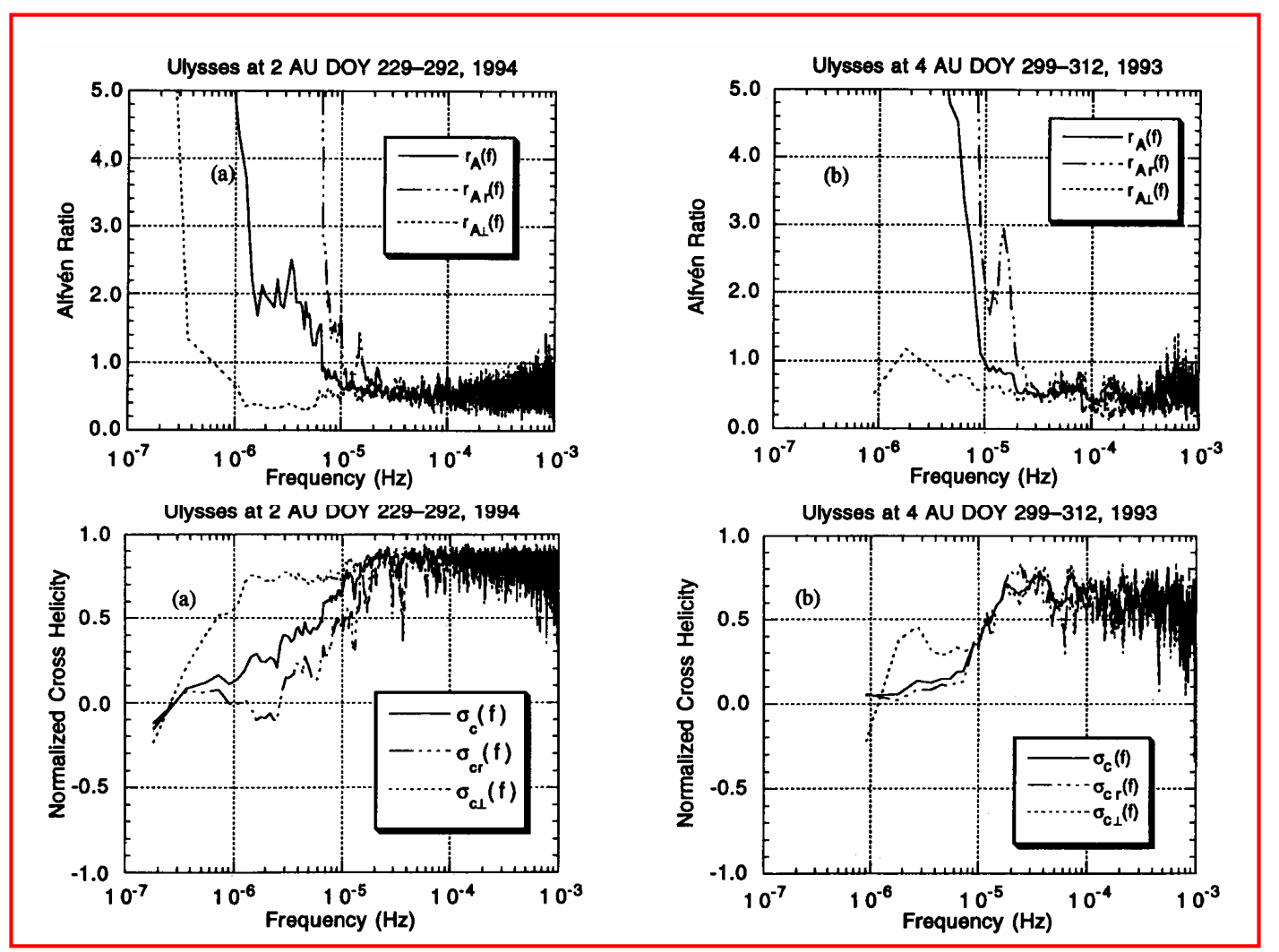

Figure 54: Normalized cross-helicity and Alfvén ratio at 2 and 4 AU, as observed by Ulysses at $-80^{\circ}$ and $-40^{\circ}$ latitude, respectively (adopted from Goldstein et al., 1995a, (c) 1995 American Geophysical Union, reproduced by permission of American Geophysical Union). 
Roberto Bruno and Vincenzo Carbone

However, by the time Ulysses reaches to $4 \mathrm{AU}, \sigma_{\mathrm{c} \perp}$ has strongly decreased as expected while $r_{\mathrm{A} \perp}$ gets closer to 1, making the situation even less clear. Anyhow, these results suggest that the situation at $2 \mathrm{AU}$ and, even more at $4 \mathrm{AU}$, can be considered as an evolution of what Helios 2 recorded in the ecliptic at shorter heliocentric distance. Ulysses observations at $2 \mathrm{AU}$ resemble more the turbulence conditions observed by Helios at $0.9 \mathrm{AU}$ rather than at $0.3 \mathrm{AU}$.

Bavassano et al. (2000a) studied in detail the evolution of the power $e^{+}$and $e^{-}$associated with outward $\delta z^{+}$and inward $\delta z^{-}$Alfvénic fluctuations, respectively. The study referred to the polar regions, during the wind expansion between 1.4 and $4.3 \mathrm{AU}$. These authors analyzed $1 \mathrm{~h}$ variances of $\delta z^{ \pm}$and found two different regimes, as shown in Figure 55. Inside 2.5 AU outward modes $e^{+}$decrease faster than inward modes $e^{-}$, in agreement with previous ecliptic observations performed within the trailing edge of corotating fast streams (Bruno and Bavassano, 1991; Tu and Marsch, 1990b; Grappin et al., 1989). Beyond this distance, the radial gradient of $e^{-}$becomes steeper and steeper while that of $e^{+}$remains approximately unchanged. This change in $e^{-}$is rather fast and both species keep declining with the same rate beyond $2.5 \mathrm{AU}$. The radial dependence of $e^{+}$between $r^{-1.39}$ and $r^{-1.48}$, reported by Bavassano et al. (2000a), indicate a radial decay faster than $r^{-1}$ predicted by WKB approximation. This is in agreement with the analysis performed by Forsyth et al. (1996) using magnetic field observations only.

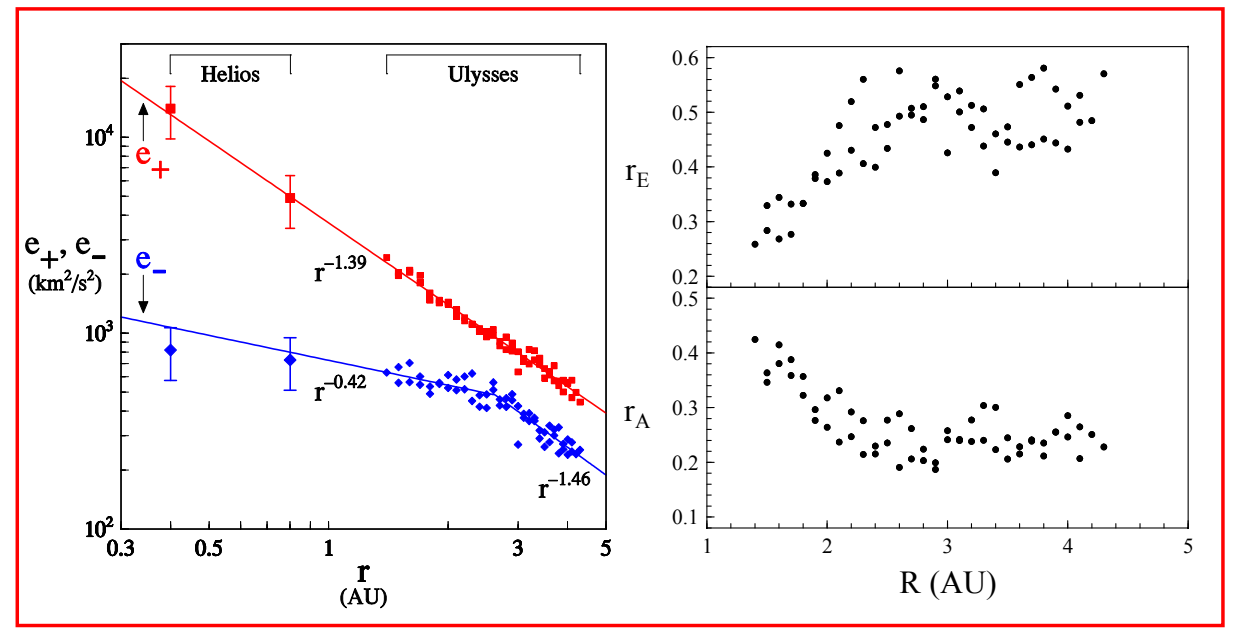

Figure 55: Left panel: values of hourly variance of $\delta z^{ \pm}$(i.e., $e^{ \pm}$) vs. heliocentric distance, as observed by Ulysses. Helios observations are shown for comparison and appear to be in good agreement. Right column: Elsässer ratio (top panel) and Alfvén ratio (bottom panel) are plotted vs. radial distance while Ulysses is embedded in the polar wind (adopted from Bavassano et al., 2000a,b, (c) 2000 American Geophysical Union, reproduced by permission of American Geophysical Union).

This different radial behavior is readily seen in the radial plot of the Elsässer ratio $r_{\mathrm{E}}$ shown in the top panel of the left column of Figure 55. Before 2.5 AU this ratio continuously grows to about 0.5 near $2.5 \mathrm{AU}$. Beyond this region, since the radial gradient of the inward and outward components is approximately the same, $r_{\mathrm{E}}$ stabilizes around 0.5 .

On the other hand, also the Alfvén ratio $r_{\mathrm{A}}$ shows a clear radial dependence that stops at about the same limit distance of 2.5 AU. In this case, $r_{\mathrm{A}}$ constantly decreases from $\sim 0.4$ at $1.4 \mathrm{AU}$ to $\sim 0.25$ at $2.5 \mathrm{AU}$, slightly fluctuating around this value for larger distances.

Another interesting feature observed in polar turbulence is unraveled by Figure 56 from Bavassano et al. (1998, 2000b). The plot shows 2D histograms of normalized cross-helicity and normalized

Living Reviews in Solar Physics

http://www . livingreviews . org/lrsp-2005-4 
residual energy (see Appendix 13.3.1 for definition) for different heliospheric regions (ecliptic wind, mid-latitude wind with strong velocity gradients, polar wind). A predominance of outward fluctuations (positive values of $\sigma_{\mathrm{c}}$ ) and of magnetic fluctuations (negative values of $\sigma_{\mathrm{r}}$ ) seems to be a general feature. It results that the most Alfvénic region is the one at high latitude and at shorter heliocentric distances. However, in all the panels there is always a relative peak at $\sigma_{\mathrm{c}} \simeq 0$ and $\sigma_{\mathrm{r}} \simeq-1$, which might well be due to magnetic structures like the MFDT found by Tu and Marsch (1991) in the ecliptic.

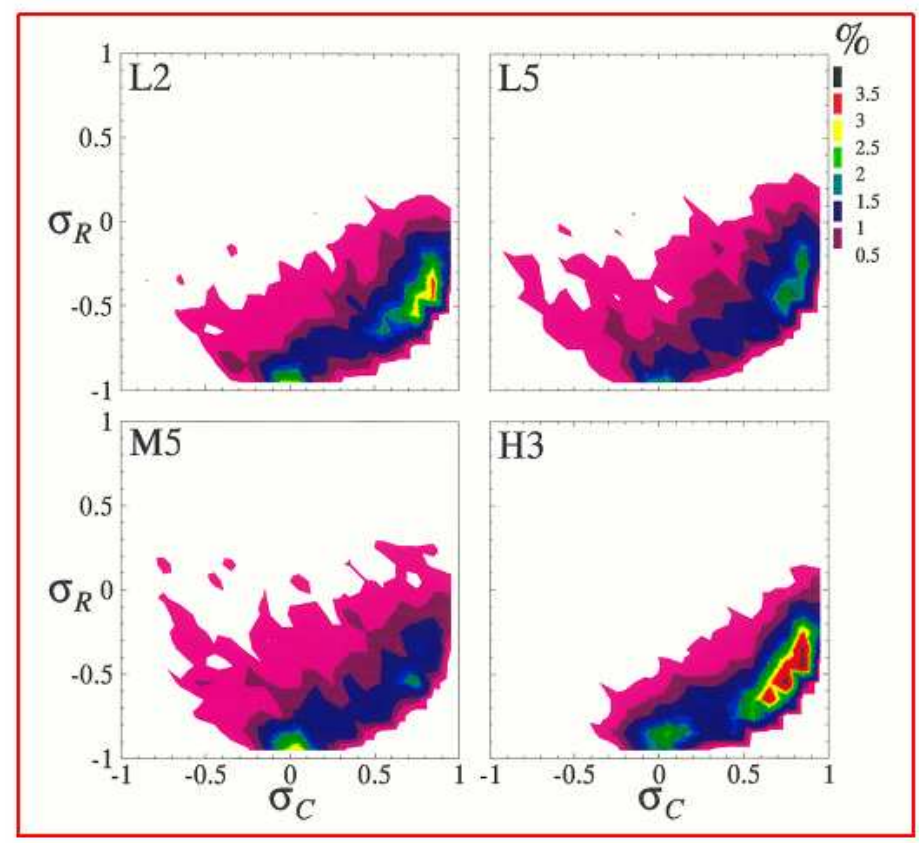

Figure 56: 2D histograms of normalized cross-helicity $\sigma_{\mathrm{c}}$ (here indicated by $\sigma_{C}$ ) and normalized residual energy $\sigma_{\mathrm{r}}$ (here indicated by $\sigma_{R}$ ) for different heliospheric regions (ecliptic wind, midlatitude wind with strong velocity gradients, polar wind) (adopted from Bavassano et al., 1998, (c) 1998 American Geophysical Union, reproduced by permission of American Geophysical Union).

In a successive paper, Bavassano et al. (2002a) tested whether or not the radial dependence observed in $e^{ \pm}$was to be completely ascribed to the radial expansion of the wind or possible latitudinal dependencies also contributed to the turbulence evolution in the polar wind.

As already discussed in the previous section, Horbury and Balogh (2001), using Ulysses data from the northern polar pass, evaluated the dependence of magnetic field power levels on solar distance and latitude using a multiple regression analysis based on Equation (37). In the Alfvénic range, the latitudinal coefficient " $C$ " for power in field components was appreciably different from 0 (around 0.3). However, this analysis was limited to magnetic field fluctuations alone and cannot be transferred sic et simpliciter to Alfvénic turbulence. In their analysis, Bavassano et al. (2002b) used the first southern and northern polar passes and removed from their dataset all intervals with large gradients in plasma velocity, and/or plasma density, and/or magnetic field magnitude, as already done in Bavassano et al. (2000a). As a matter of fact, the use of Elsässer variables (see Appendix 13.3.1) instead of magnetic field, and of selected data samples, leads to very small values of the latitudinal coefficient as shown in Figure 57, where different contributions are plotted with different colors and where the top panel refers to the same dataset used by Horbury and Balogh (2001), while the bottom panel refers to a dataset omni-comprehensive of south and north passages 
free of strong compressive events (Bavassano et al., 2000a). Moreover, the latitudinal effect appears to be very weak also for the data sample used by Horbury and Balogh (2001), although this is the sample with the largest value of the " $C$ " coefficient.

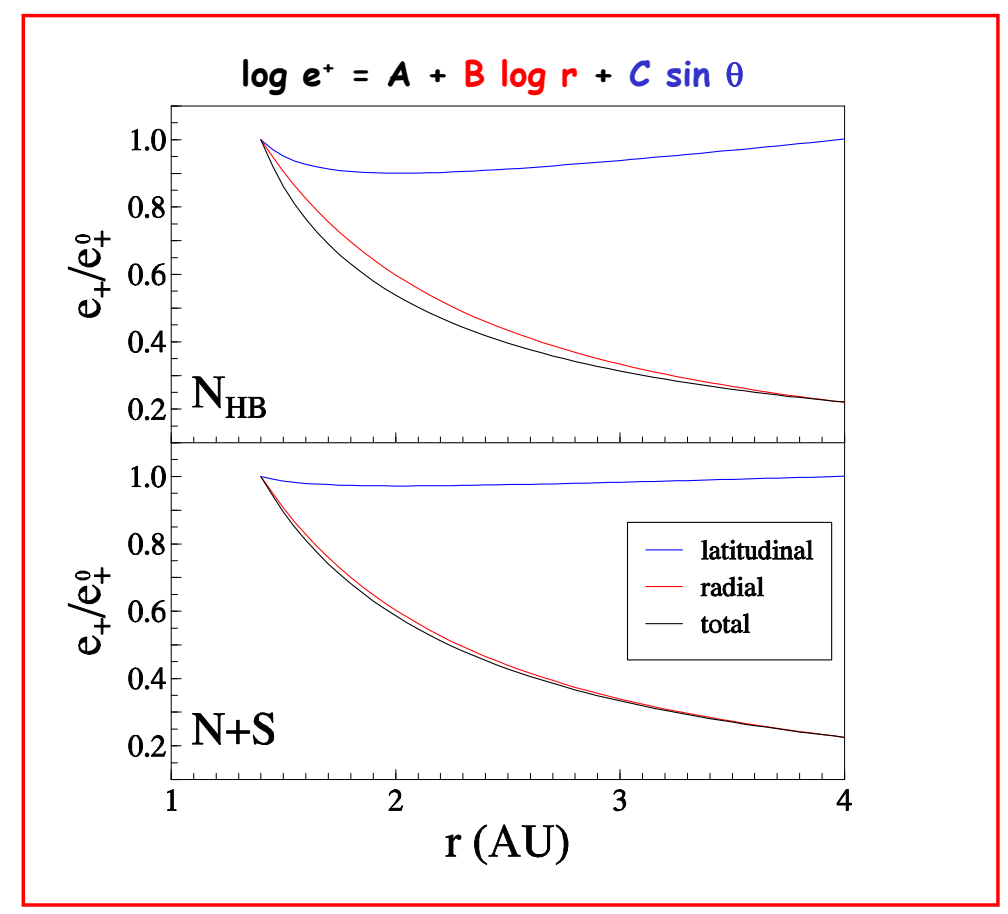

Figure 57: Results from the multiple regression analysis showing radial and latitudinal dependence of the power $e^{+}$associated with outward modes (see Appendix 13.3.1). The top panel refers to the same dataset used by Horbury and Balogh (2001). The bottom panel refers to a dataset omnicomprehensive of south and north passages free of strong compressive events (Bavassano et al., 2000a). Values of $e_{+}$have been normalized to the value $e_{+}{ }^{\circ}$ assumed by this parameter at $1.4 \mathrm{AU}$, closest approach to the Sun. The black line is the total regression, the blue line is the latitudinal contribution and the red line is the radial contribution (adopted from Bavassano et al., 2002a, (C) 2002 American Geophysical Union, reproduced by permission of American Geophysical Union).

A further argument in favor of radial vs. latitudinal dependence is represented by the comparison of the radial gradient of $e^{+}$in different regions, in the ecliptic and in the polar wind. These results, shown in Figure 58, provide the radial slopes for $e^{+}$(red squares) and $e^{-}$(blue diamonds) in different regions. The first three columns (labeled EQ) summarize ecliptic results obtained with different values of an upper limit (TBN) for relative fluctuations of density and magnetic intensity. The last two columns (labeled POL) refer to the results for polar turbulence (north and south passes) outside and inside 2.6 AU, respectively. A general agreement exists between slopes in ecliptic and in polar wind with no significant role left for latitude, the only exception being $e^{-}$in the region inside 2.6 AU. The behavior of the inward component cannot be explained by a simple power law over the range of distances explored by Ulysses. Moreover, a possible latitudinal effect has been clearly rejected by the results from a multiple regression analysis performed by Bavassano et al. (2002a) similar to that reported above for $e^{+}$.

Living Reviews in Solar Physics

http://www . livingreviews . org/lrsp-2005-4 


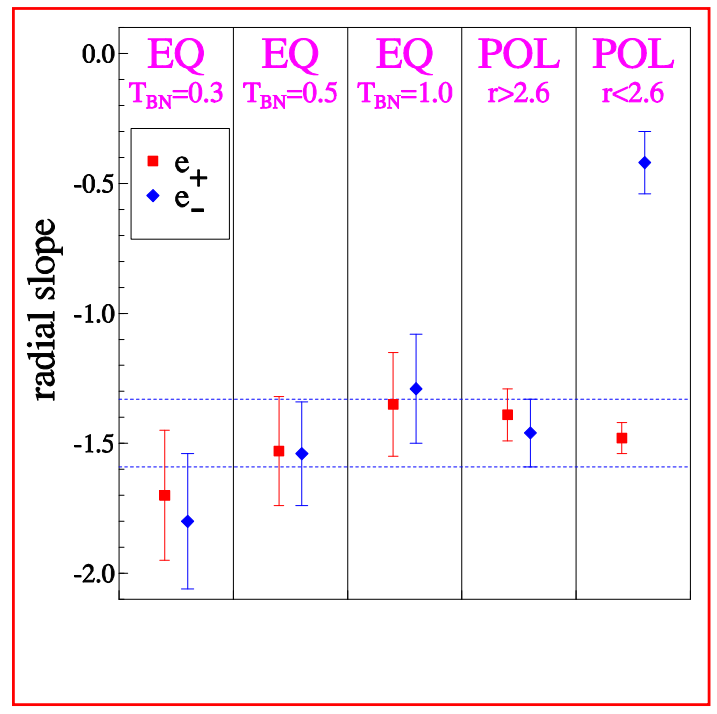

Figure 58: $e^{+}$(red) and $e^{-}$(blue) radial gradient for different latitudinal regions of the solar wind. The first three columns, labeled EQ, refer to ecliptic observations obtained with different values of the upper limit of TBN defined as the relative fluctuations of density and magnetic intensity. The last two columns, labeled POL, refer to observations of polar turbulence outside and inside 2.6 AU, respectively (adopted from Bavassano et al., 2001, (c) 2001 American Geophysical Union, reproduced by permission of American Geophysical Union).

\section{$5 \quad$ Numerical Simulations}

Numerical simulations currently represent one of the main source of information about non-linear evolution of fluid flows. The actual super-computers are now powerful enough to simulate equations (NS or MHD) that describe turbulent flows with Reynolds numbers of the order of $10^{4}$ in twodimensional configurations, or $10^{3}$ in three-dimensional one. Of course, we are far from achieving realistic values, but now we are able to investigate turbulence with an inertial range extended for more than one decade. Rather the main source of difficulties to get results from numerical simulations is the fact that they are made under some obvious constraints (say boundary conditions, equations to be simulated, etc.), mainly dictated by the limited physical description that we are able to use when numerical simulations are made, compared with the extreme richness of the phenomena involved: numerical simulations, even in standard conditions, are used tout court as models for the solar wind behavior. Perhaps the only exception, to our knowledge, is the attempt to describe the effects of the solar wind expansion on turbulence evolution by Velli et al. (1989, 1990). Even with this far too pessimistic point of view, used here solely as a few words of caution, simulations in some cases were able to reproduce some phenomena observed in the solar wind.

Nevertheless, numerical simulations have been playing a key role, and will continue to do so in our seeking an understanding of turbulent flows. Numerical simulations allows us to get information that cannot be obtained in laboratory. For example, high resolution numerical simulations provide information at every point on a grid and, for some times, about basic vector quantities and their derivatives. The number of degree of freedom required to resolve the smaller scales is proportional to a power of the Reynolds number, say to $R e^{9 / 4}$, although the dynamically relevant number of modes may be much less. Then one of the main challenge remaining is how to handle and analyze the huge data files produced by large simulations (of the order of Terabytes). Actually a lot of 
Roberto Bruno and Vincenzo Carbone

papers appeared in literature on computer simulations related to MHD turbulence. The interested reader can look at the book by Biskamp (1993) and the reviews by Pouquet $(1993,1996)$.

\subsection{Local production of Alfvénic turbulence in the ecliptic}

The discovery of the strong correlation between velocity and magnetic field fluctuations has represented the motivation for some MHD numerical simulations, aimed to confirm the conjecture by Dobrowolny et al. (1980b). The high level of correlation seems to be due to a kind of selforganization (dynamical alignment) of MHD turbulence, generated by the natural evolution of MHD towards the strongest attractive fixed point of equations (Ting et al., 1986; Carbone and Veltri, 1987, 1992). Numerical simulations (Carbone and Veltri, 1992; Ting et al., 1986) confirmed this conjecture, say MHD turbulence spontaneously can tends towards a state were correlation increases, that is, the quantity $\sigma_{\mathrm{c}}=2 H_{\mathrm{c}} / E$, where $H_{\mathrm{c}}$ is the cross-helicity and $E$ the total energy of the flow (see Appendix 13.1), tends to be maximal.

The picture of the evolution of incompressible MHD turbulence, which comes out is rather nice but solar wind turbulence displays a more complicated behavior. In particular, as we have reported above, observations seems to point out that solar wind evolves in the opposite way. The correlation is high near the Sun, at larger radial distances, from 1 to $10 \mathrm{AU}$ the correlation is progressively lower, while the level in fluctuations of mass density and magnetic field intensity increases. What is more difficult to understand is why correlation is progressively destroyed in the solar wind, while the natural evolution of MHD is towards a state of maximal normalized cross-helicity. A possible solution can be found in the fact that solar wind is neither incompressible nor statistically homogeneous, and some efforts to tentatively take into account more sophisticated effects have been made.

A mechanism, responsible for the radial evolution of turbulence, was suggested by Roberts and Goldstein (1988), Goldstein et al. (1989), and Roberts et al. $(1991,1992)$ and was based on velocity shear generation. The suggestion to adopt such a mechanism came from a detailed analysis made by Roberts et al. (1987a,b) of Helios and Voyager interplanetary observations of the radial evolution of the normalized cross-helicity $\sigma_{\mathrm{c}}$ at different time scales. Moreover, Voyager's observations showed that plasma regions, which had not experienced dynamical interactions with neighboring plasma, kept the Alfvénic character of the fluctuations at distances as far as 8 AU (Roberts et al., 1987b). In particular, the vicinity of Helios trajectory to the interplanetary current sheet, characterized by low velocity flow, suggested Roberts et al. (1991) to include in his simulations a narrow low speed flow surrounded by two high speed flows. The idea was to mimic the slow, equatorial solar wind between north and south fast polar wind. Magnetic field profile and velocity shear were reconstructed using the 6 lowest $Z^{ \pm}$Fourier modes as shown in Figure 59. An initial population of purely outward propagating Alfvénic fluctuations $\left(z^{+}\right)$was added at large $k$ and was characterized by a spectral slope of $k^{-1}$. No inward modes were present in the same range. Results of Figure 59 show that the time evolution of $z^{+}$spectrum is quite rapid at the beginning, towards a steeper spectrum, and slows down successively. At the same time, $z^{-}$modes are created by the generation mechanism at higher and higher $k$ but, along a Kolmogorov-type slope $k^{-5 / 3}$.

These results, although obtained from simulations performed using $2 \mathrm{D}$ incompressible spectral and pseudo-spectral codes, with fairly small Reynolds number of $R e \simeq 200$, were similar to the spectral evolution observed in the solar wind (Marsch and Tu, 1990a). Moreover, spatial averages across the simulation box revealed a strong cross-helicity depletion right across the slow wind, representing the heliospheric current sheet. However, magnetic field inversions and even relatively small velocity shears would largely affect an initially high Alfvénic flow (Roberts et al., 1992). However, Bavassano and Bruno (1992) studied an interaction region, repeatedly observed between 0.3 and $0.9 \mathrm{AU}$, characterized by a large velocity shear and previously thought to be a good candidate for shear generation (Bavassano and Bruno, 1989). They concluded that, even in the

Living Reviews in Solar Physics

http://www . livingreviews . org/Irsp-2005-4 


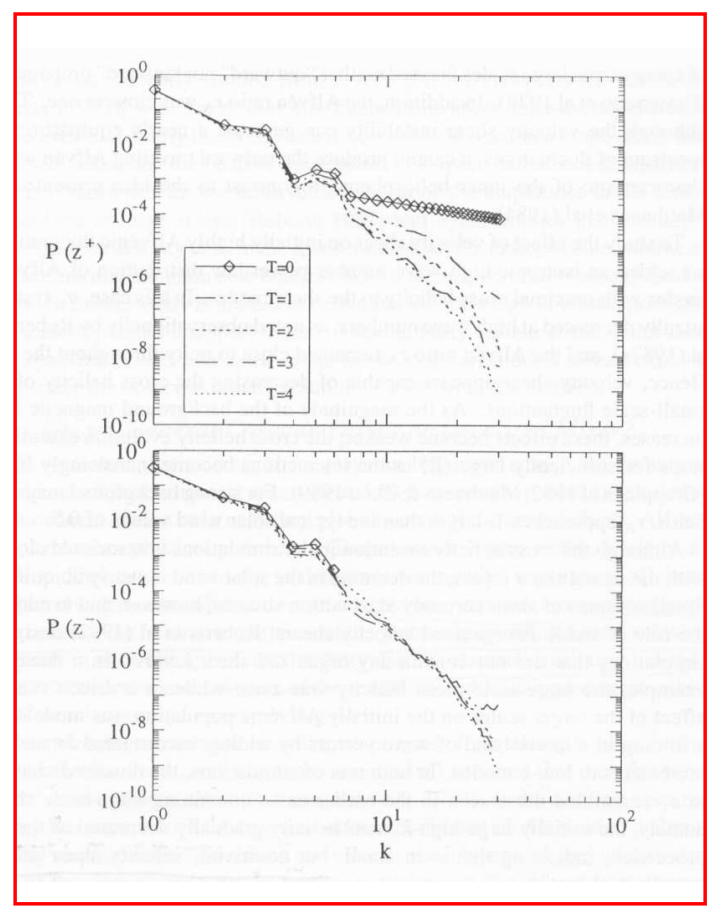

Figure 59: Time evolution of the power density spectra of $z^{+}$and $z^{-}$showing the turbulent evolution of the spectra due to velocity shear generation (adopted from Roberts et al., 1991).

hypothesis of a very fast growth of the instability, inward modes would not have had enough time to fill up the whole region as observed by Helios 2 .

The above simulations by Roberts et al. (1991) were successively implemented with a compressive pseudo-spectral code (Ghosh and Matthaeus, 1990) which provided evidence that, during this turbulence evolution, clear correlations between magnetic field magnitude and density fluctuations, and between $z^{-}$and density fluctuations should arise. However, such a clear correlation, by-product of the non-linear evolution, was not found in solar wind data (Marsch and Tu, 1993b; Bruno et al., 1996). Moreover, their results did not show the flattening of $e^{-}$spectrum at higher frequency, as observed by Helios ( $\mathrm{Tu}$ et al., 1989b). As a consequence, velocity shear alone cannot explain the whole phenomenon, other mechanisms must also play a relevant role in the evolution of interplanetary turbulence.

Compressible numerical simulations have been performed by Veltri et al. (1992) and Malara et al. $(1996,2000)$ which invoked the interactions between small scale waves and large scale magnetic field gradients and the parametric instability, as characteristic effects to reduce correlations. In a compressible, statistically inhomogeneous medium such as the heliosphere, there are many processes which tend to destroy the natural evolution toward a maximal correlation, typical of standard MHD. In such a medium an Alfvén wave is subject to parametric decay instability (Viñas and Goldstein, 1991; Del Zanna et al., 2001; Del Zanna, 2001), which means that the mother wave decays in two modes: i) a compressive mode that dissipates energy because of the steepening effect, and ii) a backscattered Alfvénic mode with lower amplitude and frequency. Malara et al. (1996) showed that in a compressible medium, the correlation between the velocity and the magnetic field fluctuations is reduced because of the generation of the backward propagating Alfvénic fluctuations, and of a compressive component of turbulence, characterized by density fluctuations $\delta \rho \neq 0$ 
and magnetic intensity fluctuations $\delta|\mathbf{B}| \neq 0$.

From a technical point of view it is worthwhile to remark that, when a large scale field which varies on a narrow region is introduced (typically a tanh-like field), periodic boundaries conditions should be used with some care. Roberts et al. $(1991,1992)$ used a double shear layer, while Malara et al. (1992) introduced an interesting numerical technique based on both the glue between two simulation boxes and a Chebyshev expansion, to maintain a single shear layer, say non periodic boundary conditions, and an increased resolution where the shear layer exists.

Grappin et al. (1992) observed that the solar wind expansion increases the lengths normal to the radial direction, thus producing an effect similar to a kind of inverse energy cascade. This effect perhaps should be able to compete with the turbulent cascade which transfers energy to small scales, thus stopping the non-linear interactions. In absence of non-linear interactions, the natural tendency towards an increase of $\sigma_{\mathrm{c}}$ is stopped.

A numerical model treating the evolution of $e^{+}$and $e^{-}$, including parametric decay of $e^{+}$, was presented by Marsch and Tu (1993a). The parametric decay source term was added in order to reproduce the decreasing cross-helicity observed during the wind expansion. As a matter of fact, the cascade process, when spectrum equations for both $e^{+}$and $e^{-}$are included and solved self-consistently, can only steepen the spectra at high frequency. Results from this model, shown in Figure 60, partially reproduce the observed evolution of the normalized cross-helicity. While the radial evolution of $e^{+}$is correctly reproduced, the behavior of $e^{-}$shows an over-production of inward modes between 0.6 and $0.8 \mathrm{AU}$ probably due to an overestimation of the strength of the pump-wave. However, the model is applied to the situation observed by Helios at 0.3 AU where a rather flat $e^{-}$spectrum already exists.

\subsection{Local production of Alfvénic turbulence at high latitude}

An interesting solution to the radial behavior of the minority modes might be represented by local generation mechanisms, like parametric decay (Malara et al., 2001; Del Zanna et al., 2001), which might saturate and be inhibited beyond 2.5 AU.

Parametric instability has been studied in a variety of situations depending on the value of the plasma $\beta$ (among others Sagdeev and Galeev, 1969; Goldstein, 1978; Hoshino and Goldstein, 1989; Malara and Velli, 1996). Malara et al. (2000) and Del Zanna et al. (2001) recently studied the non-linear growth of parametric decay of a broadband Alfvén wave, and showed that the final state strongly depends on the value of the plasma $\beta$ (thermal to magnetic pressure ratio). For $\beta<1$ the instability completely destroys the initial Alfvénic correlation. For $\beta \sim 1$ (a value close to solar wind conditions) the instability is not able to go beyond some limit in the disruption of the initial correlation between velocity and magnetic field fluctuations, and the final state is $\sigma_{\mathrm{c}} \sim 0.5$ as observed in the solar wind (see Section 4.2).

These authors solved numerically the fully compressible, non-linear MHD equations in a onedimensional configuration using a pseudo-spectral numerical code. The simulation starts with a non-monochromatic, large amplitude Alfvén wave polarized on the $y z$ plane, propagating in a uniform background magnetic field. Successively, the instability was triggered by adding some noise of the order $10^{-6}$ to the initial density level.

During the first part of the evolution of the instability the amplitude of unstable modes is small and, consequently, non-linear couplings are negligible. A subsequent exponential growth, predicted by the linear theory, increases the level of both $e^{-}$and density compressive fluctuations. During the second part of the development of the instability, non-linear couplings are not longer disregardable and their effect is firstly to slow down the exponential growth of unstable modes and then to saturate the instability to a level that depends on the value of the plasma $\beta$.

Spectra of $e^{ \pm}$are shown in Figure 61 for different times during the development of the instability. At the beginning the spectrum of the mother-wave is peaked at $k=10$, and before the instability

Living Reviews in Solar Physics

http: //www . livingreviews . org/lrsp-2005-4 


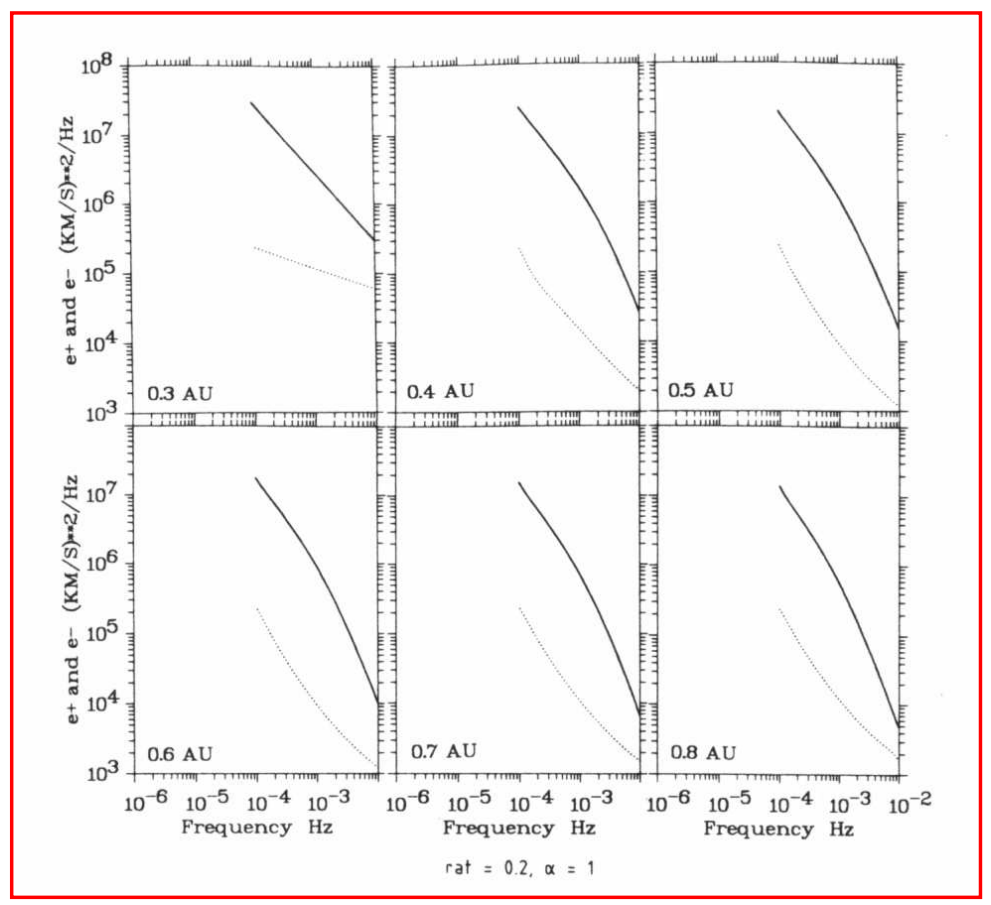

Figure 60: Radial evolution of $e^{+}$and $e^{-}$spectra obtained from the Marsch and Tu (1993a) model, in which a parametric decay source term was added to the Tu's model (Tu et al., 1984) that was, in turn, extended by including both spectrum equations for $e^{+}$and $e^{-}$and solved them self-consistently (adopted from Marsch and Tu, 1993a, (c) 1993 American Geophysical Union, reproduced by permission of American Geophysical Union). 
saturation $(t \leq 35)$ the back-scattered $e^{-}$and the density fluctuations $e^{\rho}$ are peaked at $k=1$ and $k=11$, respectively. After saturation, as the run goes on, the spectrum of $e^{-}$approaches that of $e^{+}$towards a common final state characterized by a Kolmogorov-like spectrum and $e^{+}$slightly larger than $e^{-}$.

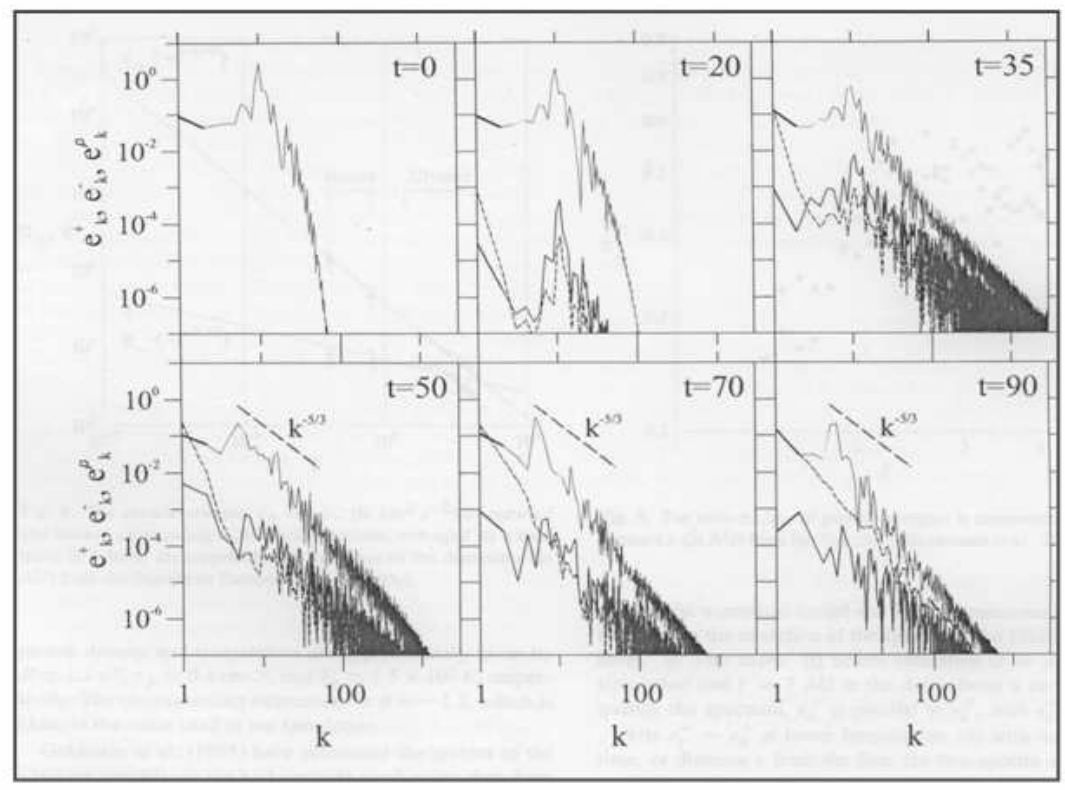

Figure 61: Spectra of $e^{+}$(thick line), $e^{-}$(dashed line), and $e^{\rho}$ (thin line) are shown for 6 different times during the development of the instability. For $t \geq 50$ a typical Kolmogorov slope appears. These results refer to $\beta=1$ (figure adopted from Malara et al., 2001).

The behavior of outward and inward modes, density and magnetic magnitude variances and the normalized cross-helicity $\sigma_{\mathrm{c}}$ is summarized in the left column of Figure 62. The evolution of $\sigma_{\mathrm{c}}$, when the instability reaches saturation, can be qualitatively compared with Ulysses observations (courtesy of B. Bavassano) in the right panel of the same figure, which shows a similar trend.

Obviously, making this comparison, one has to take into account that this model has strong limitations like the presence of a peak in $e^{+}$not observed in real polar turbulence. Another limitation, partly due to dissipation that has to be included in the model, is that the spectra obtained at the end of the instability growth are steeper than those observed in the solar wind. Finally, a further limitation is represented by the fact that this code is $1 \mathrm{D}$.

In addition, Umeki and Terasawa (1992) studying the non-linear evolution of a large-amplitude incoherent Alfvén wave via $1 \mathrm{D}$ magnetohydrodynamic simulations, reported that while in a low beta plasma $(\beta \approx 0.2)$ the growth of backscattered Alfvén waves, which are opposite in helicity and propagation direction from the original Alfvén waves, could be clearly detected, in a high beta plasma $(\beta \approx 2)$ there was no production of backscattered Alfvén waves. Consequently, although numerical results obtained by Malara et al. (2001) are very encouraging, the high beta plasma $(\beta \approx 2)$, characteristic of fast polar wind at solar minimum, plays against a relevant role of parametric instability in developing solar wind turbulence as observed by Ulysses. However, these simulations do remain an important step forward towards the understanding of turbulent evolution in the polar wind until other mechanisms will be found to be active enough to justify the observations shown in Figure 55.

Living Reviews in Solar Physics

http://www. livingreviews . org/lrsp-2005-4 

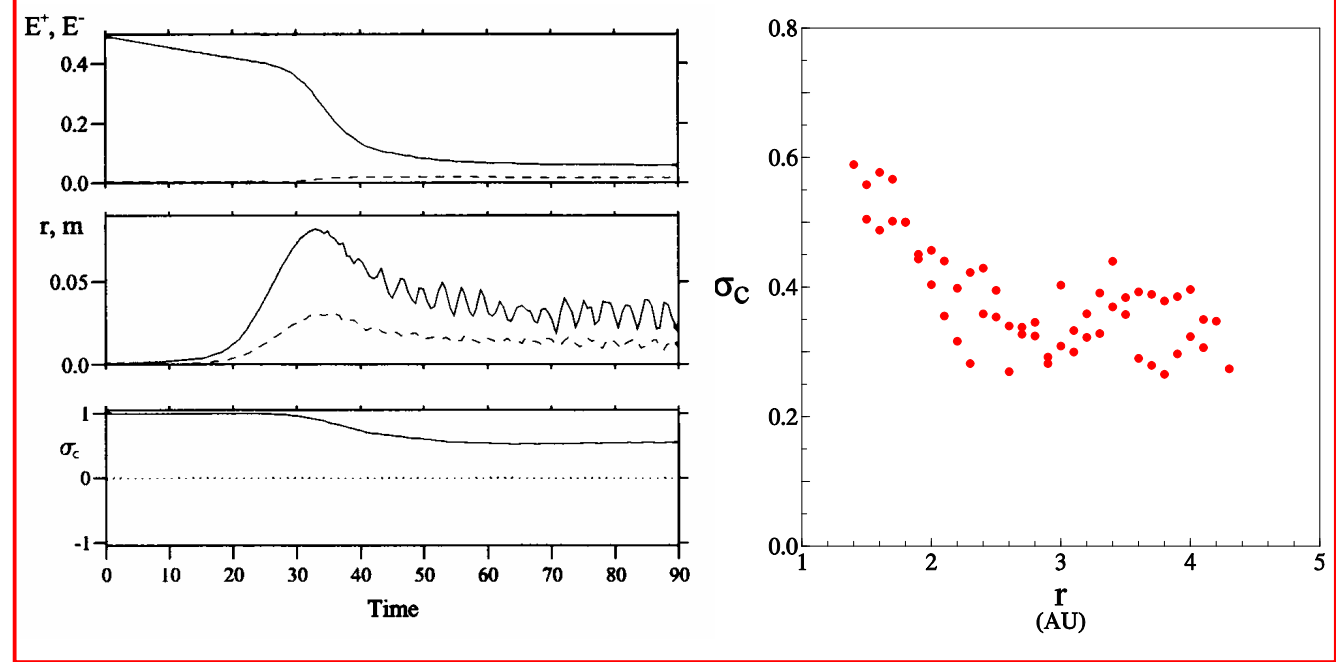

Figure 62: Top left panel: time evolution of $e^{+}$(solid line) and $e^{-}$(dashed line). Middle left panel: density (solid line) and magnetic magnitude (dashed line) variances. Bottom left panel: normalized cross helicity $\sigma_{\mathrm{c}}$. Right panel: Ulysses observations of $\sigma_{\mathrm{c}}$ radial evolution within the polar wind (left column is from Malara et al., 2001, right panel is a courtesy of B. Bavassano).

\section{Compressive Turbulence}

Interplanetary medium is slightly compressive, magnetic field intensity and proton number density experience fluctuations over all scales and the compression depends on both the scale and the nature of the wind. As a matter of fact, slow wind is generally more compressive than fast wind, as shown in Figure 63 where, following Bavassano et al. (1982a) and Bruno and Bavassano (1991), we report the ratio between the power density associated with magnetic field intensity fluctuations and that associated with the fluctuations of the three components. In addition, as already shown by Bavassano et al. (1982a), this parameter increases with heliocentric distance for both fast and slow wind as shown in the bottom panel, where the ratio between the compression at $0.9 \mathrm{AU}$ and that at $0.3 \mathrm{AU}$ is generally greater than 1 . It is also interesting to notice that within the Alfvénic fast wind, the lowest compression is observed in the middle frequency range, roughly between $10^{-4}-10^{-3} \mathrm{~Hz}$. On the other hand, this frequency range has already been recognized as the most Alfvénic one, within the inner heliosphere (Bruno et al., 1996).

As a matter of fact, it seems that high Alfvénicity is correlated with low compressibility of the medium (Bruno and Bavassano, 1991; Klein et al., 1993; Bruno and Bavassano, 1993) although compressibility is not the only cause for a low Alfvénicity (Roberts et al., 1991, 1992; Roberts, 1992).

The radial dependence of the normalized number density fluctuations $\delta n / n$ for the inner and outer heliosphere were studied by Grappin et al. (1990) and Roberts et al. (1987b) for the hourly frequency range, but no clear radial trend emerged from these studies. However, interesting enough, Grappin et al. (1990) found that values of $e^{-}$were closely associated with enhancements of $\delta n / n$ on scales longer than $1 \mathrm{~h}$.

On the other hand, a spectral analysis of proton number density, magnetic field intensity, and proton temperature performed by Marsch and Tu (1990b) and Tu et al. (1991) in the inner heliosphere, separately for fast and slow wind (see Figure 64), showed that normalized spectra of 


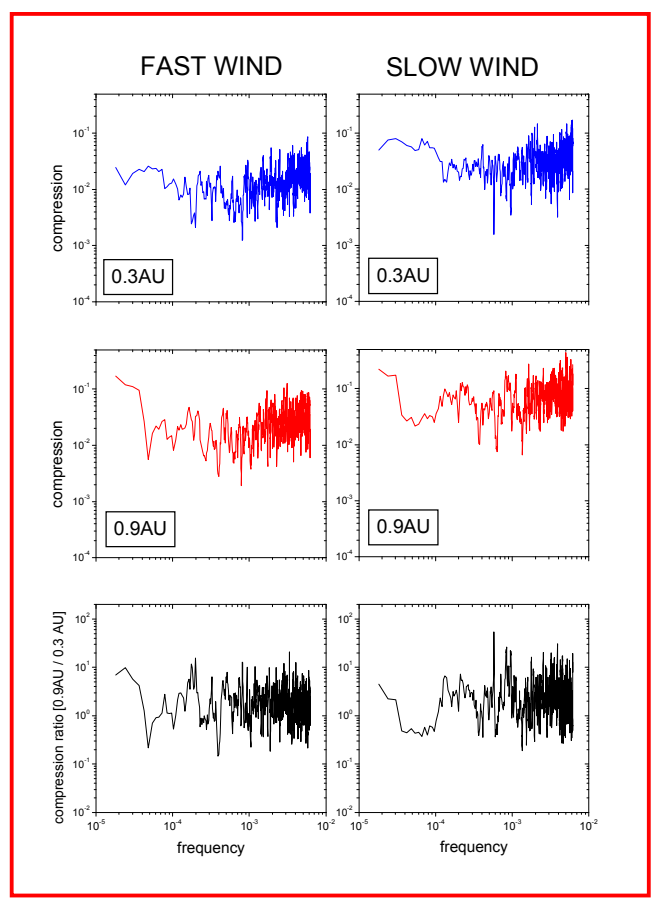

Figure 63: The first two rows show magnetic field compression (see text for definition) for fast (left column) and slow (right column) wind at $0.3 \mathrm{AU}$ (upper row) and 0.9 AU (middle row). The bottom panels show the ratio between compression at $0.9 \mathrm{AU}$ and compression at $0.3 \mathrm{AU}$. This ratio is generally greater than 1 for both fast and slow wind. 
the above parameters within slow wind were only marginally dependent on the radial distance. On the contrary, within fast wind, magnetic field and proton density normalized spectra showed not only a clear radial dependence but also similar level of power for $k<4 \cdot 10^{-4} \mathrm{~km} \mathrm{~s}^{-1}$. For larger $k$ these spectra show a flattening that becomes steeper for increasing distance, as was already found by Bavassano et al. (1982b) for magnetic field intensity. Normalized temperature spectra does not suffer any radial dependence neither in slow wind nor in fast wind.

Spectral index is around $-5 / 3$ for all the spectra in slow wind while, fast wind spectral index is around $-5 / 3$ for $k<4 \cdot 10^{-4} \mathrm{~km}^{-1}$ and slightly less steep for larger wave numbers.

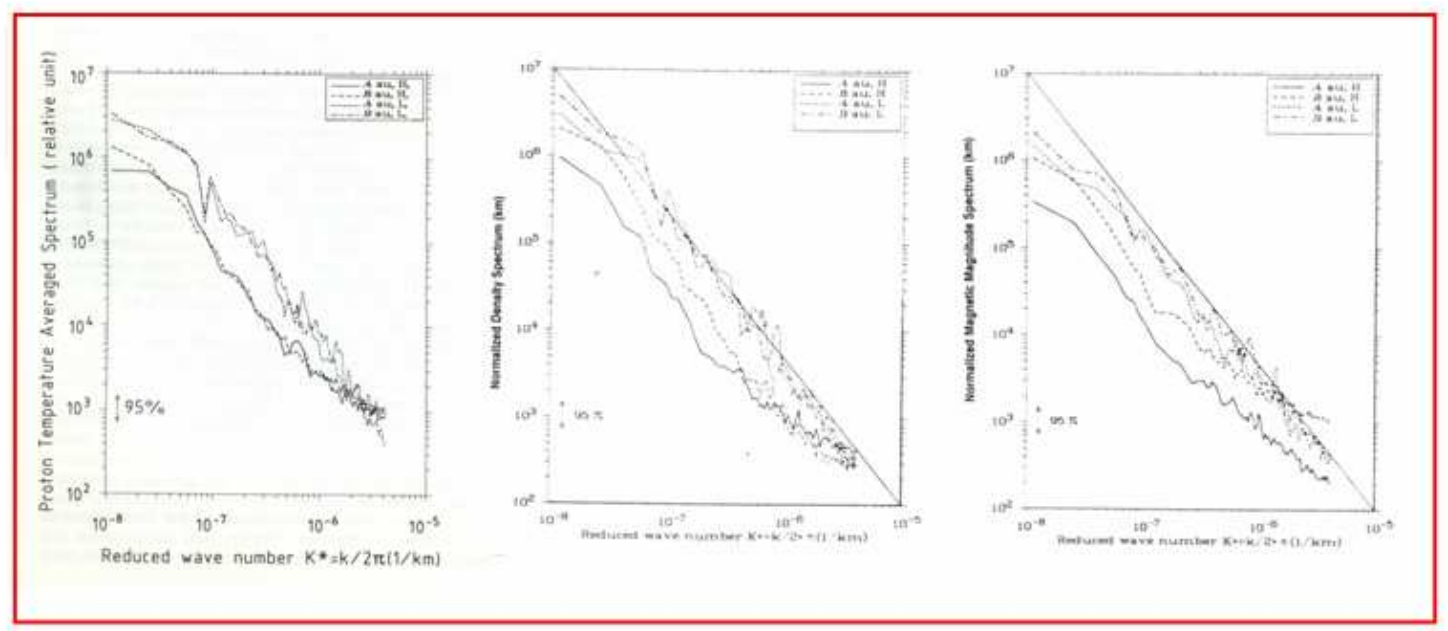

Figure 64: From left to right: normalized spectra of proton temperature (adopted from Tu et al., 1991), number density, and magnetic field intensity fluctuations (adopted from Marsch and Tu, 1990b, (c) 1990 American Geophysical Union, reproduced by permission of American Geophysical Union) Different lines refer to different heliocentric distances for both slow and fast wind.

\subsection{On the nature of compressive Turbulence}

Considerable efforts, both theoretical and observational, have been made in order to disclose the nature of compressive fluctuations. It has been proposed (Montgomery et al., 1987; Matthaeus and Brown, 1988; Zank et al., 1990; Zank and Matthaeus, 1990; Matthaeus et al., 1991; Zank and Matthaeus, 1992) that most of compressive fluctuations observed in the solar wind could be accounted for by the Nearly Incompressible (NI) model. Within the framework of this model, (Montgomery et al., 1987) showed that a spectrum of small scale density fluctuations follows a $k^{-5 / 3}$ when the spectrum of magnetic field fluctuations follows the same scaling. Moreover, it was showed (Matthaeus and Brown, 1988; Zank and Matthaeus, 1992) that if compressible MHD equations are expanded in terms of small turbulent sonic Mach numbers, pressure balanced structures, Alfvénic and magnetosonic fluctuations naturally arise as solutions and, in particular, the RMS of small density fluctuations would scale like $M^{2}$, being $M=\delta v / C_{\mathrm{s}}$ the turbulent sonic Mach number, $\delta v$ the RMS of velocity fluctuations and $C_{\mathrm{s}}$ the sound speed. In addition, if heat conduction is allowed in the approximation, temperature fluctuations dominate over magnetic and density fluctuations, temperature and density are anticorrelated and would scale like $M$. However, in spite of some examples supporting this theory (Matthaeus et al., 1991 reported 13\% of cases satisfied the requirements of NI-theory), wider statistical studies, conducted by Tu and Marsch (1994), Bavassano et al. (1995) and Bavassano and Bruno (1995), showed that NI theory is not 
applicable sic et simpliciter to the solar wind. The reason might be in the fact that interplanetary medium is highly inhomogeneous because of the presence of an underlying structure convected by the wind. As a matter of fact, Thieme et al. (1989) showed evidence for the presence of time intervals characterized by clear anti-correlation between kinetic pressure and magnetic pressure while the total pressure remained fairly constant. These pressure balance structures were for the first time observed by Burlaga and Ogilvie (1970) for a time scale of roughly one to two hours. Later on, Vellante and Lazarus (1987) reported strong evidence for anti-correlation between field intensity and proton density, and between plasma and field pressure on time scales up to $10 \mathrm{~h}$. The anti-correlation between kinetic and magnetic pressure is usually interpreted as indicative of the presence of a pressure balance structure since slow magnetosonic modes are readily damped (Barnes, 1979).

These features, observed also in their dataset, were taken by Thieme et al. (1989) as evidence of stationary spatial structures which were supposed to be remnants of coronal structures convected by the wind. Different values assumed by plasma and field parameters within each structure were interpreted as a signature characterizing that particular structure and not destroyed during the expansion. These intervals, identifiable in Figure 65 by vertical dashed lines, were characterized by pressure balance and a clear anti-correlation between magnetic field intensity and temperature.

These structures were finally related to the fine ray-like structures or plumes associated with the underlying cromospheric network and interpreted as the signature of interplanetary flow-tubes. The estimated dimension of these structures, back projected onto the Sun, suggested that they over-expand in the solar wind.

The idea of filamentary structures in the solar wind dates back to Parker (1963), followed by other authors like McCracken and Ness (1966), Siscoe et al. (1968), and more recently re-proposed in literature with new evidences (see Section 9). These interplanetary flow tubes would be of different sizes, ranging from minutes to several hours and would be separated from each other by tangential discontinuities and characterized by different values of plasma parameters and a different magnetic field orientation and intensity. This kind of scenario, because of some similarity to a bunch of tangled, smoking "spaghetti" lifted by a fork, was then named "spaghetti-model".

A spectral analysis performed by Marsch and $\mathrm{Tu}(1993 \mathrm{a})$ in the frequency range $6 \cdot 10^{-3}-6 \cdot 10^{-6}$ showed that the nature and intensity of compressive fluctuations systematically vary with the stream structure. They concluded that compressive fluctuations are a complex superposition of magnetoacoustic fluctuations and pressure balance structures whose origin might be local, due to stream dynamical interaction, or of coronal origin related to the flow tube structure. These results are shown in Figure 66 where the correlation coefficient between number density $n$ and total pressure $P_{\text {tot }}$ (indicated with the symbols $p_{\mathrm{T}}$ in the figure), and between kinetic pressure $P_{k}$ and magnetic pressure $P_{\mathrm{m}}$ (indicated with the symbols $p_{k}$ and $p_{b}$, respectively) is plotted for both Helios s/c relatively to fast wind. Positive values of correlation coefficients $C\left(n, p_{\mathrm{T}}\right)$ and $C\left(p_{k}, p_{b}\right)$ identify magnetosonic waves, while positive values of $C\left(n, p_{\mathrm{T}}\right)$ and negative values of $C\left(p_{k}, p_{b}\right)$ identify pressure balance structures. The purest examples of each category are located at the upper left and right corners.

Following these observations, Tu and Marsch (1994) proposed a model in which fluctuations in temperature, density, and field directly derive from an ensemble of small amplitude pressure balanced structures and small amplitude fast perpendicular magnetosonic waves. These last ones should be generated by the dynamical interaction between adjacent flow tubes due to the expansion and, eventually, they would experience also a non-linear cascade process to smaller scales. This model was able to reproduce most of the correlations described by Marsch and Tu (1993a) for fast wind.

Later on, Bavassano et al. (1996a) tried to characterize compressive fluctuations in terms of their polytropic index, which resulted to be a useful tool to study small scale variations in the solar wind. These authors followed the definition of polytropic fluid given by Chandrasekhar (1967): "a

Living Reviews in Solar Physics

http: //www. livingreviews . org/lrsp-2005-4 


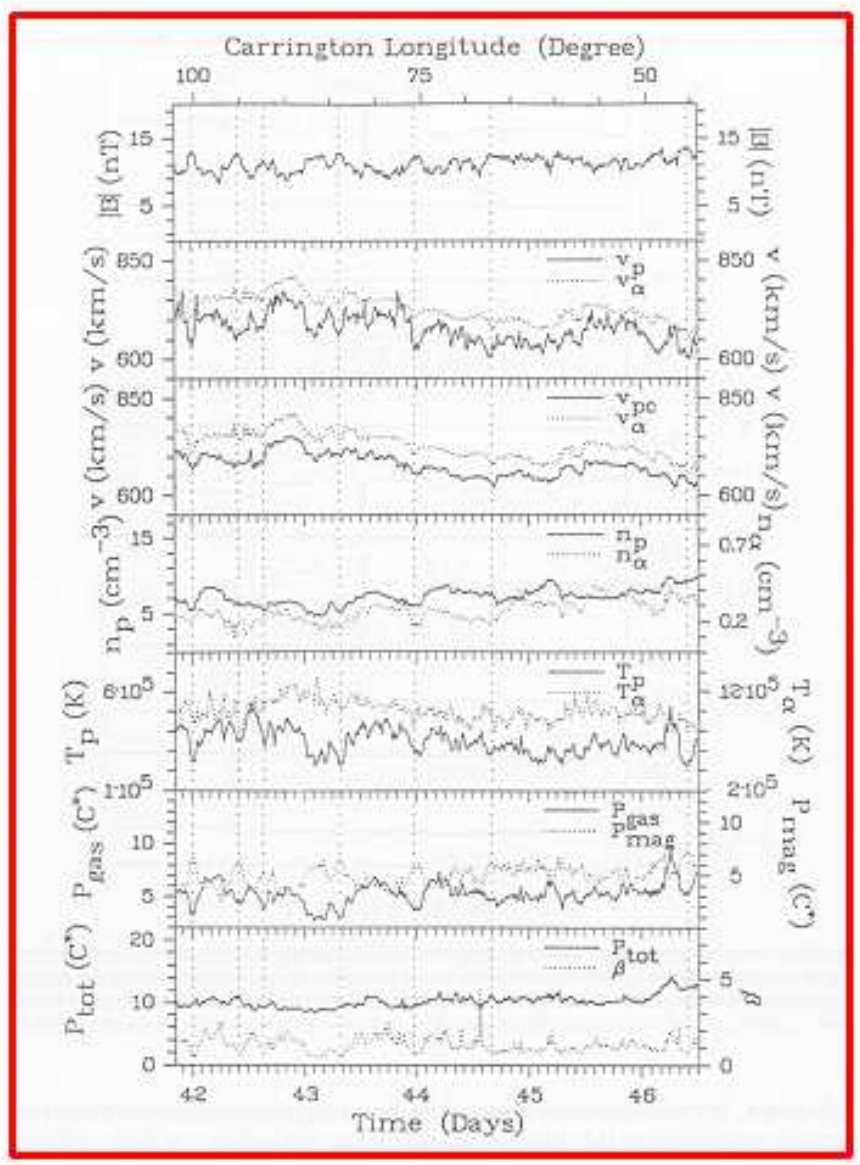

Figure 65: From top to bottom: field intensity $|\mathbf{B}|$; proton and alpha particle velocity $v_{p}$ and $v_{\alpha}$; corrected proton velocity $v_{p c}=v_{p}-\delta v_{A}$, where $v_{A}$ is the Alfvén speed; proton and alpha number density $n_{p}$ and $n_{\alpha}$; proton and alpha temperature $T_{p}$ and $T_{\alpha}$; kinetic and magnetic pressure $P_{k}$ and $P_{m}$, which the authors call $P_{\text {gas }}$ and $P_{\text {mag }}$; total pressure $P_{\text {tot }}$ and $\beta=P_{\text {gas }} / P_{\text {mag }}$ (adopted from Thieme et al., 1989). 


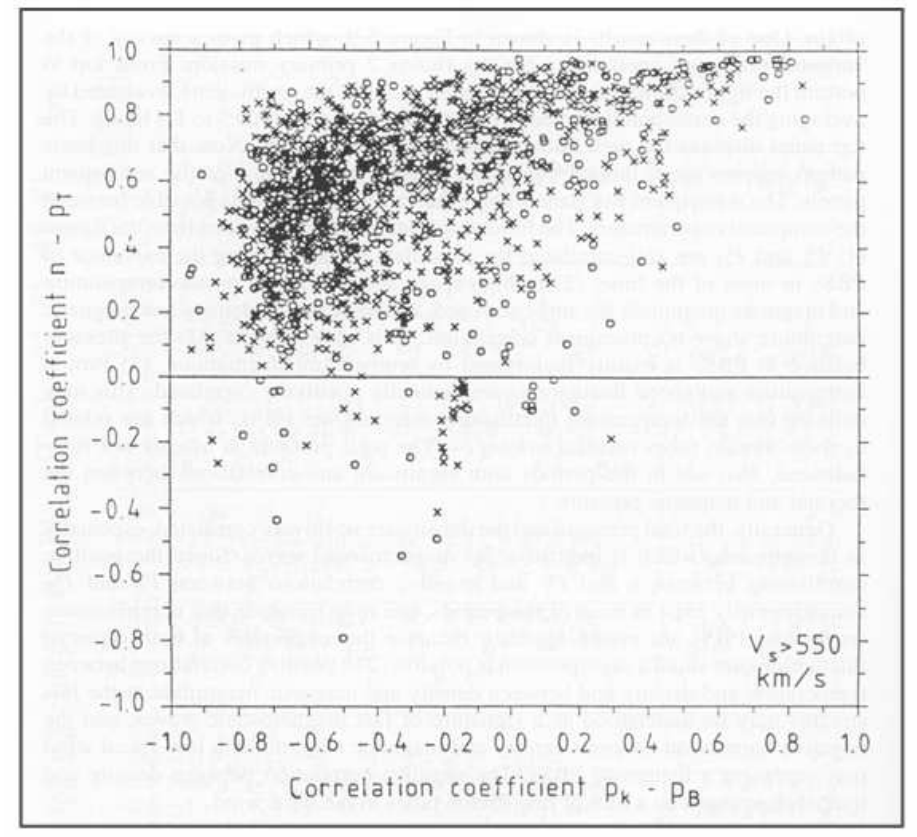

Figure 66: Correlation coefficient between number density $n$ and total pressure $p_{\mathrm{T}}$ plotted vs. the correlation coefficient between kinetic pressure and magnetic pressure for both Helios relatively to fast wind (adopted from Marsch and Tu, 1993b).

polytropic change is a quasi-static change of state carried out in such a way that the specific heat remains constant (at some prescribed value) during the entire process". For such a variation of state the adiabatic laws are still valid provided that the adiabatic index $\gamma$ is replaced by a new adiabatic index $\gamma^{\prime}=\left(c_{P}-c\right) /\left(c_{V}-c\right)$ where $c$ is the specific heat of the polytropic variation, and $c_{P}$ and $c_{V}$ are the specific heat at constant pressure and constant volume, respectively. This similarity is lost if we adopt the definition given by Courant and Friedrichs (1976), for whom a fluid is polytropic if its internal energy is proportional to the temperature. Since no restriction applies to the specific heats, relations between temperature, density, and pressure do not have a simple form as in Chandrasekhar approach (Zank and Matthaeus, 1991). Bavassano et al. (1996a) recovered the polytropic index from the relation between density $n$ and temperature $T$ changes for the selected scale $T n^{1-\gamma^{\prime}}=$ const. and used it to determine whether changes in density and temperature were isobaric $\left(\gamma^{\prime}=0\right)$, isothermal $\left(\gamma^{\prime}=1\right)$, adiabatic $\left(\gamma^{\prime}=\gamma\right)$, or isochoric $\left(\gamma^{\prime}=\infty\right)$. Although the role of the magnetic field was neglected, reliable conclusions could be obtained whenever the above relations between temperature and density were strikingly clear. These authors found intervals characterized by variations at constant thermal pressure $P$. They interpreted these intervals as a subset of total-pressure balanced structures where the equilibrium was assured by the thermal component only, perhaps tiny flow tubes like those described by Thieme et al. (1989) and Tu and Marsch (1994). Adiabatic changes were probably related to magnetosonic waves excited by contiguous flow tubes ( $\mathrm{Tu}$ and Marsch, 1994). Proton temperature changes at almost constant density were preferentially found in fast wind, close to the Sun. These regions were characterized by values of $B$ and $N$ remarkable stable and by strong Alfvénic fluctuations (Bruno et al., 1985). Thus, they suggested that these temperature changes could be remnants of thermal features already established at the base of the corona.

Thus, the polytropic index offers a very simple way to identify basic properties of solar wind

Living Reviews in Solar Physics

http: //www . livingreviews . org/lrsp-2005-4 
fluctuations, provided that the magnetic field does not play a major role.

\subsection{Compressive turbulence in the polar wind}

Compressive fluctuations in high latitude solar wind have been extensively studied by Bavassano et al. (2004) looking at the relationship between different parameters of the solar wind and comparing these results with predictions by existing models.

These authors indicated with $N, P_{m}, P_{k}$, and $P_{t}$ the proton number density $n$, magnetic pressure, kinetic pressure and total pressure $\left(P_{t o t}=P_{m}+P_{k}\right)$, respectively, and computed correlation coefficients $\rho$ between these parameters. Figure 67 clearly shows that a pronounced positive correlation for $N-P_{t}$ and a negative pronounced correlation for $P_{m}-P_{k}$ is a constant feature of the observed compressive fluctuations. In particular, the correlation for $N-P_{t}$ is especially strong within polar regions at small heliocentric distance. In mid-latitude regions the correlation weakens, while almost disappears at low latitudes. In the case of $P_{m}-P_{k}$, the anticorrelation remains strong throughout the whole latitudinal excursion. For polar wind the anticorrelation appears to be less strong at small distances, just where the $N-P_{t}$ correlation is highest.

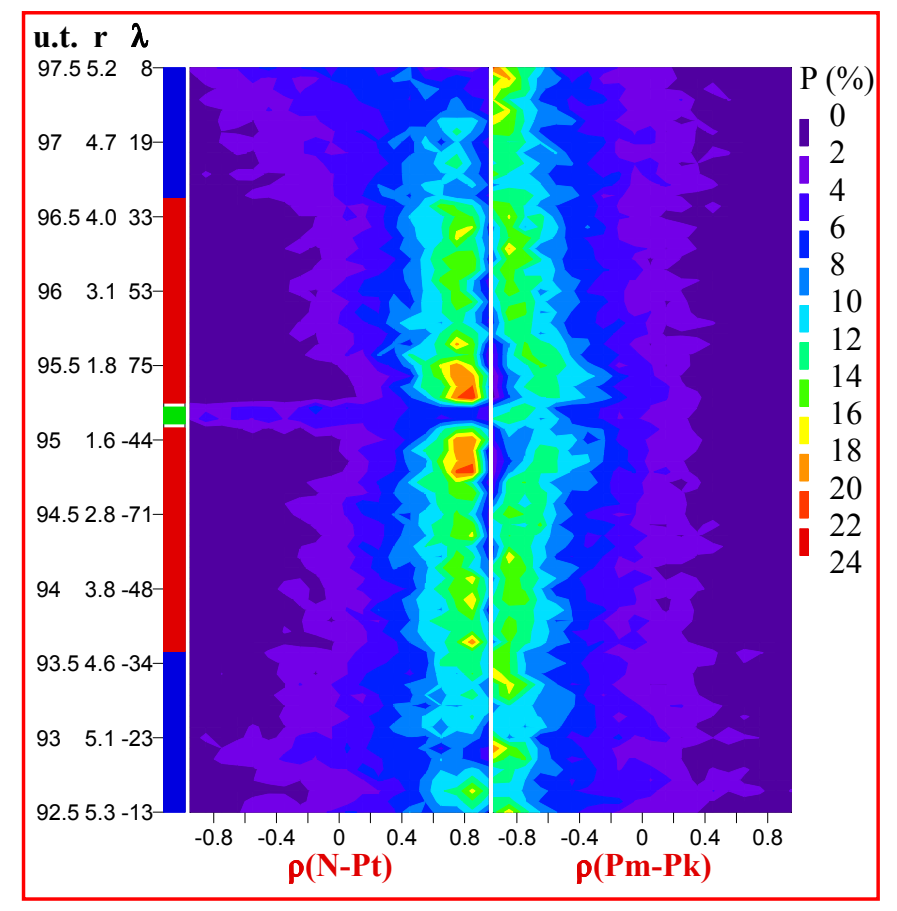

Figure 67: Histograms of $\rho\left(N-P_{t}\right)$ and $\rho\left(P_{m}-P_{k}\right)$ per solar rotation. The color bar on the left side indicates polar (red), mid-latitude (blue), and low latitude (green) phases. Moreover, universal time UT, heliocentric distance, and heliographic latitude are also indicated on the left side of the plot. Occurrence frequency is indicated by the color bar shown on the right hand side of the Figure (figure adopted from Bavassano et al., 2004).

The role played by density and temperature in the anticorrelation between magnetic and thermal pressures is investigated in Figure 68, where the magnetic field magnitude is directly compared with proton density and temperature. As regards the polar regions, a strong B-T anticorrelation is clearly apparent at all distances (right panel). For B-N an anticorrelation tends to emerge when 
solar distance increases. This means that the magnetic-thermal pressure anticorrelation is mostly due to an anticorrelation of the magnetic field fluctuations with respect to temperature fluctuations, rather than density (see, e.g., Bavassano et al., 1996a,b). Outside polar regions the situation appears in part reversed, with a stronger role for the B-N anticorrelation.

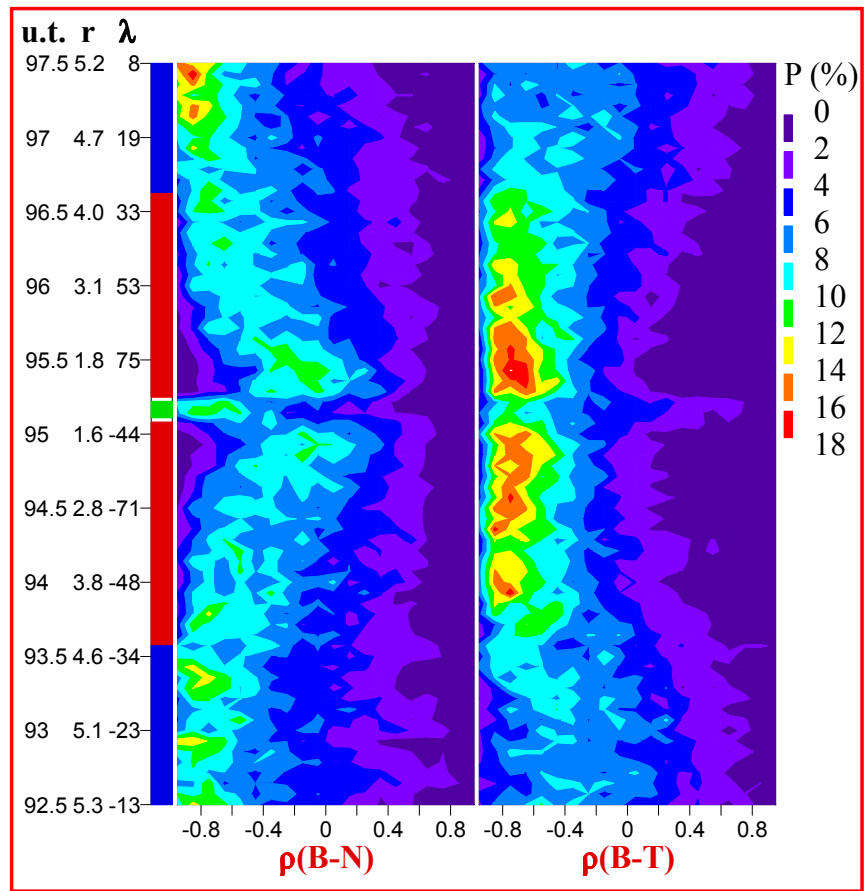

Figure 68: Solar rotation histograms of $B-N$ and $B-T$ in the same format of Figure 67 (figure adopted from Bavassano et al., 2004).

In Figure 69 scatter plots of total pressure vs. density fluctuations are used to test a model by Tu and Marsch (1994), based on the hypothesis that the compressive fluctuations observed in solar wind are mainly due to a mixture of pressure-balanced structures (PBS) and fast magnetosonic waves $(\mathrm{W})$. Waves can only contribute to total pressure fluctuations while both waves and pressurebalanced structures may contribute to density fluctuations. A tunable parameter in the model is the relative PBS/W contribution to density fluctuations $\alpha$. Straight lines in Figure 69 indicate the model predictions for different values of $\alpha$. It is easily seen that for all polar wind samples the great majority of experimental data fall in the $\alpha>1$ region. Thus, pressure-balanced structures appear to play a major role with respect to magnetosonic waves. This is a feature already observed by Helios in the ecliptic wind ( $\mathrm{Tu}$ and Marsch, 1994), although in a less pronounced way. Different panels of Figure 69 refer to different heliocentric distances within the polar wind. Namely, going from P1 to $\mathrm{P} 4$ is equivalent to move from 1.4 to $4 \mathrm{AU}$. A comparison between these panels indicates that the observed distribution tends to shift towards higher values of $\alpha$ (i.e., pressure-balanced structures become increasingly important), which probably is a radial distance effect.

Finally, the relative density fluctuations dependence on the turbulent Mach number M (the ratio between velocity fluctuation amplitude and sound speed) is shown in Figure 70. The aim is to look for the presence, in the observed fluctuations, of nearly incompressible MHD behaviors. In the framework of the NI theory (Zank and Matthaeus, 1991, 1993) two different scalings for the relative density fluctuations are possible, as $M$ or as $M^{2}$, depending on the role that thermal

Living Reviews in Solar Physics

http: //www. livingreviews.org/lrsp-2005-4 


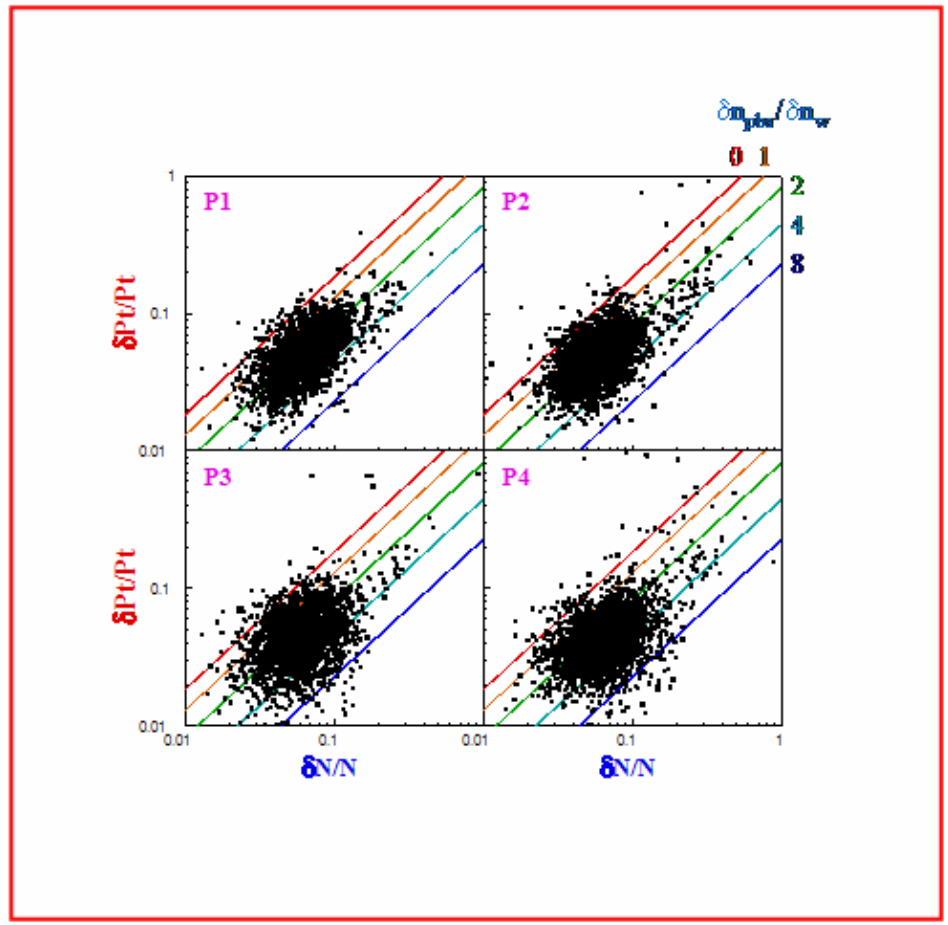

Figure 69: Scatter plots of the relative amplitudes of total pressure vs. density fluctuations for polar wind samples P1 to P4. Straight lines indicate the Tu and Marsch (1994) model predictions for different values of $\alpha$, the relative PBS/W contribution to density fluctuations (figure adopted from Bavassano et al., 2004). 
conduction effects may play in the plasma under study (namely a heat-fluctuation-dominated or a heat-fluctuation-modified behavior, respectively). These scalings are shown in Figure 70 as solid (for $M$ ) and dashed (for $M^{2}$ ) lines.

It is clearly seen that for all the polar wind samples no clear trend emerges in the data. Thus, NI-MHD effects do not seem to play a relevant role in driving the polar wind fluctuations. This confirms previous results in the ecliptic by Helios in the inner heliosphere (Bavassano et al., 1995; Bavassano and Bruno, 1995) and by Voyagers in the outer heliosphere (Matthaeus et al., 1991). It is worthy of note that, apart from the lack of NI trends, the experimental data from Ulysses, Voyagers, and Helios missions in all cases exhibit quite similar distributions. In other words, for different heliospheric regions, solar wind regimes, and solar activity conditions, the behavior of the compressive fluctuations in terms of relative density fluctuations and turbulent Mach numbers seems almost to be an invariant feature.

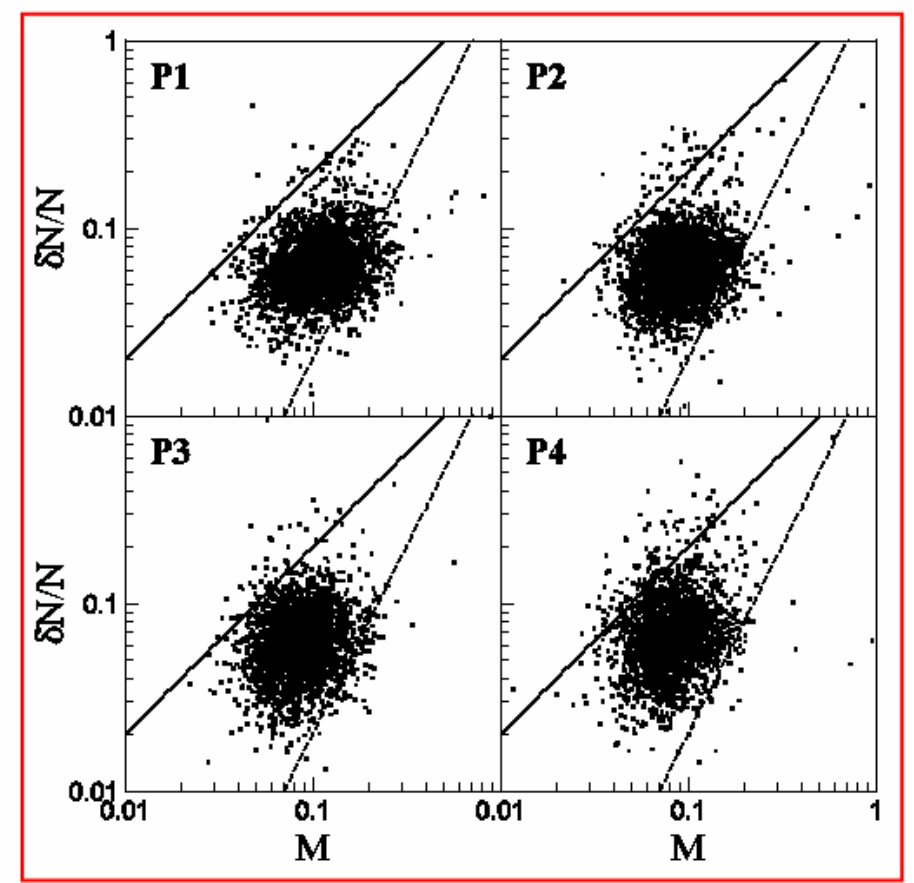

Figure 70: Relative amplitude of density fluctuations vs. turbulent Mach number for polar wind. Solid and dashed lines indicate the $M$ and $M^{2}$ scalings, respectively (figure adopted from Bavassano et al., 2004).

The above observations fully support the view that compressive fluctuations in high latitude solar wind are a mixture of MHD modes and pressure balanced structures. It has to be reminded that previous studies (McComas et al., 1995, 1996; Reisenfeld et al., 1999) indicated a relevant presence of pressure balanced structures at hourly scales. Moreover, nearly-incompressible (see Section 6.1) effects do not seem to play any relevant role. Thus, polar observations do not show major differences when compared with ecliptic observations in fast wind, the only possible difference being a major role of pressure balanced structures.

Living Reviews in Solar Physics

http: //www. livingreviews . org/lrsp-2005-4 


\subsection{The effect of compressive phenomena on Alfvénic correlations}

A lack of $\delta \mathbf{V}-\delta \mathbf{B}$ correlation does not strictly indicate a lack of Alfvénic fluctuations since a superposition of both outward and inward oriented fluctuations of the same amplitude would produce a very low correlation as well. In addition, the rather complicated scenario we observe at the base of the corona, where complicated kinetic and magnetic phenomena contribute to the birth of the wind, suggest that the imprints of such a structured corona is carried away by the wind during its expansion. At this point, we would expect that solar wind fluctuations would not solely due to the ubiquitous Alfvénic and other MHD propagating modes but also to an underlying structure convected by the wind, not necessarily characterized by Alfvén-like correlations. Moreover, dynamical interactions between fast and slow wind, built up during the expansion, contribute to increase the compressibility of the medium.

It has been suggested that disturbances of the mean magnetic field intensity and plasma density act destructively on $\delta \mathbf{V}-\delta \mathbf{B}$ correlation. Bruno and Bavassano (1993) analyzed the loss of the Alfvénic character of interplanetary fluctuations in the inner heliosphere within the low frequency part of the Alfvénic range, i.e., between 2 and $10 \mathrm{~h}$. In Figure 71, from their work, shows the wind speed profile, $\sigma_{\mathrm{c}}$, the correlation coefficients, phase and coherence for the three components (see Appendix 13.2.1), the angle between magnetic field and velocity minimum variance directions, and the heliocentric distance. Magnetic field sectors were rectified (see Appendix 13.3) and magnetic field and velocity components were rotated into the magnetic field minimum variance reference system (see Appendix 15). Although the three components behave in a similar way, the most Alfvénic ones are the two components $Y$ and $Z$ transverse to the minimum variance component $X$. As a matter of fact, for an Alfvén mode we would expect high $\delta V-\delta B$ correlation, a phase close to zero for outward waves and a high coherence. Moreover, it is rather clear that the most Alfvénic intervals are located within the trailing edges of high velocity streams. However, as the radial distance increases, the Alfvénic character of the fluctuations decreases and the angle $\Theta_{b v}$ increases. The same authors found that high values of $\Theta_{b v}$ are associated with low values of $\sigma_{\mathrm{c}}$ and correspond to the most compressive intervals. They concluded that the depletion of the Alfvénic character of the fluctuations, within the hourly frequency range, might be driven by the interaction with static structures or magnetosonic perturbations able to modify the homogeneity of the background medium on spatial scales comparable to the wavelength of the Alfvénic fluctuations. A successive paper by Klein et al. (1993) showed that the $\delta \mathbf{V}-\delta \mathbf{B}$ decoupling increases with the plasma $\beta$, suggesting that in regions where the local magnetic field is less relevant, compressive events play a major role in this phenomenon. 


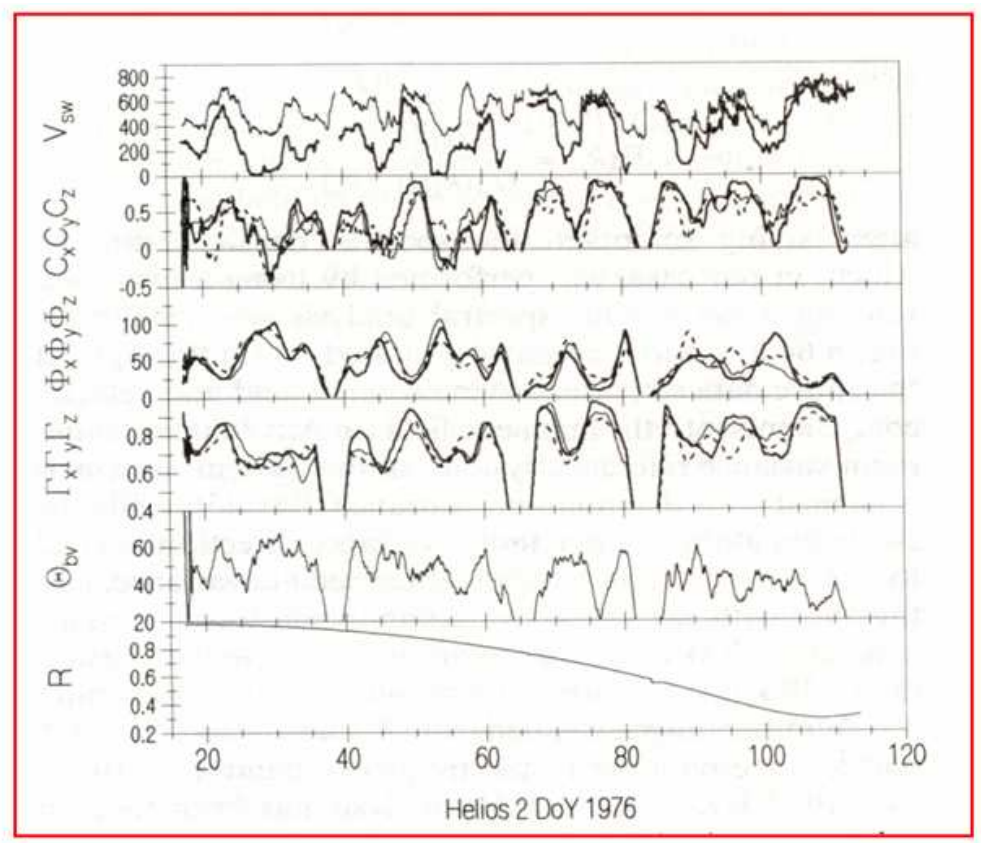

Figure 71: Wind speed profile $V$ and $\left|\sigma_{\mathrm{c}}\right| V$ are shown in the top panel. The lower three panels refer to correlation coefficient, phase angle and coherence for the three components of $\delta V$ and $\delta B$ fluctuations, respectively. The successive panel indicates the value of the angle between magnetic field and velocity fluctuations minimum variance directions. The bottom panel refers to the heliocentric distance (adopted from Bruno and Bavassano, 1993). 


\section{A Natural Wind Tunnel}

The solar wind has been used as a wind tunnel by L.F. Burlaga who, at the beginning of the '90s, started to investigate anomalous fluctuations (Burlaga, 1991a,b,c, 1995) as observed by measurements in the outer heliosphere by the Voyager spacecraft. In $1991 \mathrm{E}$. Marsch, in a review on solar wind turbulence given at the Solar Wind Seven conference (Marsch, 1992), underlined the importance of investigating scaling laws in the solar wind and we like to report his sentence: "The recent work by Burlaga (1991a,b) opens in my mind a very promising avenue to analyze and understand solar wind turbulence from a new theoretical vantage point. ... This approach may also be useful for MHD turbulence. Possible connections between intermittent turbulence and deterministic chaos have recently been investigated ... We are still waiting for applications of these modern concepts of chaos theory to solar wind MHD fluctuations." (cf. Marsch, 1992, page 503). A few years later Carbone (1993) and, independently, Biskamp (1993) faced the question of anomalous scaling from a theoretical point of view. More than ten years later the investigation of statistical mechanics of MHD turbulence from one side, and of low-frequency solar wind turbulence on the other side, has produced a lot of papers, and is now mature enough to be tentatively presented in a more organic way.

\subsection{Scaling exponents of structure functions}

The phenomenology of turbulence developed by Kolmogorov (1941) deals with some statistical hypotheses for fluctuations. The famous footnote remark by Landau (Landau and Lifshitz, 1971) pointed out a defect in the Kolmogorov theory, namely the fact that the theory does not take proper account of spatial fluctuations of local dissipation rate (Frisch, 1995). This led different authors to investigate the features related to scaling laws of fluctuations and, in particular, to investigate the departure from the Kolmogorov's linear scaling of the structure functions (cf. Section 2.8). An up-to-date comprehensive review of these theoretical efforts can be found in the book by Frisch (1995).

Here we are interested in understanding what we can learn from solar wind turbulence about the basic features of scaling laws for fluctuations. We use velocity and magnetic fields time series, and we investigate the scaling behavior of the high-order moments of stochastic variables defined as variations of fields separated by a time ${ }^{8}$ interval $r$. First of all, it is worthwhile to remark that scaling laws and, in particular, the exact relation (26) which defines the inertial range in fluid flows, is valid for longitudinal (streamwise) fluctuations. In common fluid flows the Kolmogorov linear scaling law is compared with the moments of longitudinal velocity differences. In the same way for the solar wind turbulence we investigate the scaling behavior of $\delta u_{r}=u(t+r)-u(t)$, where $u(t)$ represents the component of the velocity field along the radial direction. As far as the magnetic differences are concerned $\delta b_{r}=B(t+r)-B(t)$, we are free for different choices and, in some sense, this is more interesting from an experimental point of view. We can use the reference system where $B(t)$ represents the magnetic field projected along the radial direction, or the system where $B(t)$ represents the magnetic field along the local background magnetic field, or $B(t)$ represents the field along the minimum variance direction. As a different case we can simply investigate the scaling behavior of the fluctuations of the magnetic field intensity.

Let us consider the $p$-th moment of both absolute values of velocity fluctuations $R_{p}(r)=\left\langle\left|\delta u_{r}\right|^{p}\right\rangle$ and magnetic fluctuations $S_{p}(r)=\left\langle\left|\delta b_{r}\right|^{p}\right\rangle$, also called $p$-th order structure function in literature (brackets being time average). Here we use magnetic fluctuations across structures at intervals $r$ calculated by using the magnetic field intensity. Typical structure functions of magnetic field fluctuations, for two different values of $p$, for both a slow wind and a fast wind at $0.9 \mathrm{AU}$, are

\footnotetext{
${ }^{8}$ Since the solar wind moves at supersonic speed $V_{\mathrm{sw}}$, the usual Taylor's hypothesis is verified, and we can get information on spatial $(\ell)$ scaling laws by using time differences $r=\ell / V_{\mathrm{sw}}$.
} 
shown in Figures 72. The magnetic field we used is that measured by Helios 2 spacecraft. Structure functions calculated for the velocity fields have roughly the same shape. Looking at these figures the three typical ranges of turbulence can be observed. Starting from low values at small scales, the structure functions increase towards a region where $S_{p} \rightarrow$ const. at the largest scales. This means that at these scales the field fluctuations are uncorrelated. A kind of "inertial range", that is a region of intermediate scales $r$ where a power law can be recognized for both $S_{p}(r) \sim r^{\zeta_{p}}$ and $R_{p}(r) \sim r^{\xi_{p}}$, is more or less visible only for the slow wind. In this range correlations exists, and we can obtain the scaling exponents $\zeta_{p}$ and $\xi_{p}$ through a simple linear fit.

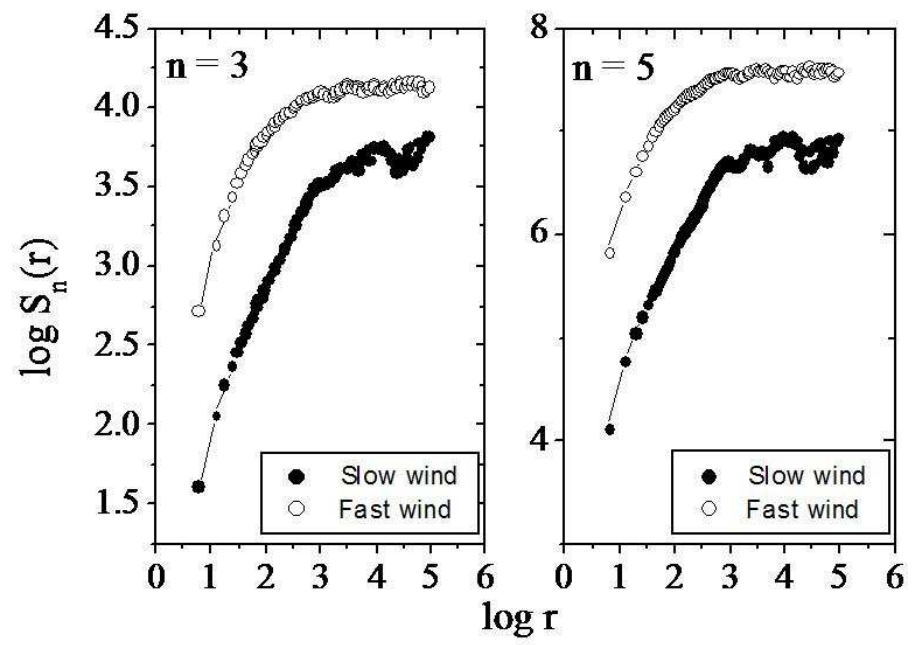

Figure 72: Structure functions for the magnetic field intensity $S_{n}(r)$ for two different orders, $n=3$ and $n=5$, for both slow wind and fast wind, as a function of the time scale $r$. Data come from Helios 2 spacecraft at 0.9 AU.

Since the inertial range is not well defined ${ }^{9}$, in order to compare scaling exponents of the solar wind turbulent fluctuations with other experiments, it is perhaps better to try to recover exponents using the Extended Self-Similarity (ESS), introduced some time ago by Benzi et al. (1993), and used here as a tool to determine relative scaling exponents. In the fluid-like case, the 3-th order structure function can be regarded as a generalized scaling using the inverse of Equation (26) or of Equation (27) (Politano et al., 1998b). Then, we can plot the $p$-th order structure function vs. the 3 -th order to recover at least relative scaling exponents $\zeta_{p} / \zeta_{3}$ and $\xi_{p} / \xi_{3}$. Quite surprisingly (see Figure 73 ), we find that the range where a power law can be recovered extends well beyond the inertial range, covering almost all the experimental range. In the fluid case the scaling exponents which can be obtained through ESS at low or moderate Reynolds numbers, coincide with the scaling exponents obtained for high Reynolds, where the inertial range is very well defined Benzi et al. (1993). This is due to the fact that, since the $4 / 5$-law, by definition $\zeta_{3}=1$ in the inertial range (Frisch, 1995), whatever its extension might be. In our case scaling exponents obtained through ESS can be used as a surrogate, since we cannot be sure that an inertial range exists.

It is worthwhile to remark (as shown in Figure 73) that we can introduce a general scaling

\footnotetext{
${ }^{9}$ It is worthwhile to remark that neither the fluid relation (26) nor its MHD counterpart (27) are satisfied in the solar wind. Namely there is not any extended range of scales, from which we can derive scaling exponents, where the above relations which formally define the inertial range are verified. Here we are in a situation similar to a low-Reynolds number fluid flow.
}

Living Reviews in Solar Physics

http: //www . livingreviews . org/lrsp-2005-4 


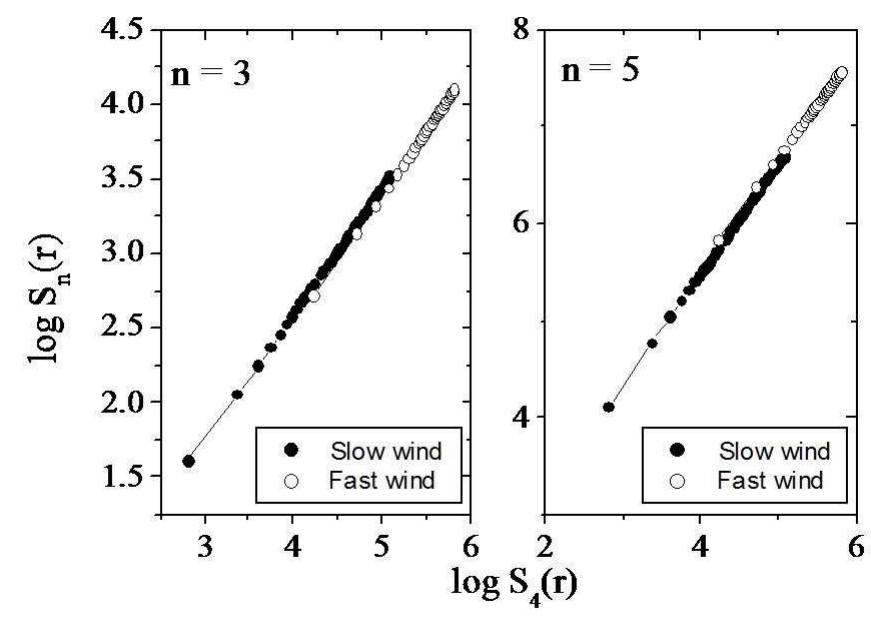

Figure 73: Structure functions $S_{n}(r)$ for two different orders, $n=3$ and $n=5$, for both slow wind and high wind, as a function of the fourth-order structure function $S_{4}(r)$. Data come from Helios 2 spacecraft at $0.9 \mathrm{AU}$.

relation between the $q$-th order velocity structure function and the $p$-th order structure function, with a relative scaling exponent $\alpha_{p}(q)$. It has been found that this relation becomes an exact relation

$$
S_{q}(r)=\left[S_{p}(r)\right]^{\alpha_{p}(q)},
$$

when the velocity structure functions are normalized to the average velocity within each period used to calculate the structure function (Carbone et al., 1996a). This is very interesting because it implies (Carbone et al., 1996a) that the above relationship is satisfied by the following probability distribution function, if we assume that odd moments are much smaller than the even ones:

$$
f\left(\delta u_{r}\right)=\int_{-\infty}^{\infty} d k e^{i k \delta u_{r}} \sum_{q=0}^{\infty} \frac{(i k)^{2 q}}{2 \pi(2 q) !}\left[S_{p}(r)\right]^{\alpha_{p}(2 q)} .
$$

That is, for each scale $r$ the knowledge of the relative scaling exponents $\alpha_{p}(q)$ completely determines the probability distribution of velocity differences as a function of a single parameter $S_{p}(r)$.

Relative scaling exponents, calculated by using data coming from Helios 2 at $0.9 \mathrm{AU}$, are reported in Table 1. As it can be seen, two main features can be noted:

i. There is a significant departure from the Kolmogorov linear scaling, that is, real scaling exponents are anomalous and seem to be non-linear functions of $p$, say $\zeta_{p} / \zeta_{3}>p / 3$ for $p<3$, while $\zeta_{p} / \zeta_{3}<p / 3$ for $p>3$. The same behavior can be observed for $\xi_{p} / \xi_{3}$. In Table 1 we report also the scaling exponents obtained in usual fluid flows for velocity and temperature, the latter being a passive scalar. Scaling exponents for velocity field are similar to scaling exponents obtained in turbulent flows on Earth, showing a kind of universality in the anomaly. This effect is commonly attributed to the phenomenon of intermittency in fully developed turbulence (Frisch, 1995). Turbulence in the solar wind is intermittent, just like its fluid counterpart on Earth.

ii. The degree of intermittency is measured through the distance between the curve $\zeta_{p} / \zeta_{3}$ and the linear scaling $p / 3$. It can be seen that magnetic field is more intermittent than velocity 
Table 1: Scaling exponents for velocity $\zeta_{p}$ and magnetic $\xi_{p}$ variables calculated through ESS. Errors represent the standard deviations of the linear fitting. The data used comes from a turbulent sample of slow wind at $0.9 \mathrm{AU}$ from Helios 2 spacecraft. As a comparison we show the normalized scaling exponents of structure functions calculated in a wind tunnel on Earth (Ruíz-Chavarría et al., 1995) for velocity and temperature. The temperature is a passive scalar in this experiment.

\begin{tabular}{lllll}
\hline$p$ & $\zeta_{p}$ & $\xi_{p}$ & $u(t)$ (fluid) & $T(t)$ (fluid) \\
\hline 1 & $0.37 \pm 0.06$ & $0.56 \pm 0.06$ & 0.37 & 0.61 \\
2 & $0.70 \pm 0.05$ & $0.83 \pm 0.05$ & 0.70 & 0.85 \\
3 & 1.00 & 1.00 & 1.00 & 1.00 \\
4 & $1.28 \pm 0.02$ & $1.14 \pm 0.02$ & 1.28 & 1.12 \\
5 & $1.54 \pm 0.03$ & $1.25 \pm 0.03$ & 1.54 & 1.21 \\
6 & $1.79 \pm 0.05$ & $1.35 \pm 0.05$ & 1.78 & 1.40 \\
\hline
\end{tabular}

field. The same difference is observed between the velocity field and a passive scalar (in our case the temperature) in ordinary fluid flows (Ruíz-Chavarría et al., 1995). That is the magnetic field, as long as intermittency properties are concerned, behaves like a passive field.

In Table 1 we show scaling exponents up to the sixth order. Actually, a question concerns the validation of high-order moments estimates, say the maximum value of the order $p$ which can be determined with a finite number of points of our dataset. As the value of $p$ increases, we need an increasing number of points for an optimal determination of the structure function (Tennekes and Wyngaard, 1972). Anomalous scaling laws are generated by rare and intense events due to singularities in the gradients: the higher their intensity the more rare these events are. Of course, when the dataset has a finite extent, the probability to get singularities stronger than a certain value approaches zero. In that case, scaling exponents $\zeta_{p}$ of order higher than a certain value look to be linear function of $p$. Actually, the structure function $S_{p}(r)$ depends on the probability distribution function $\operatorname{PDF}\left(\delta u_{r}\right)$ through

$$
S_{p}(r)=\int \delta u_{r}^{p} P D F\left(\delta u_{r}\right) d \delta u_{r},
$$

and the function $S_{p}$ is determined only when the integral converges. As $p$ increases, the function $F_{p}\left(\delta u_{r}\right)=\delta u_{r}^{p} P D F\left(\delta u_{r}\right)$ becomes more and more disturbed, with some spikes, so that the integral becomes more and more undefined, as can be seen for example in Figure 1 of the paper by Dudok de Wit (2004). A simple calculation (Dudok de Wit, 2004) for the maximum value of the order $p_{m}$ which can reliably be estimated with a given number $N$ of points in the dataset, gives the empirical criterion $p_{m} \simeq \log N$. Structure functions of order $p>p_{m}$ cannot be determined accurately.

As far as a comparison between different plasmas is concerned, the scaling exponents of magnetic structure functions, obtained from laboratory plasma experiments of a Reversed-Field Pinch at different distances from the external wall (Carbone et al., 2000) are shown in Table 2. In laboratory plasmas it is difficult to measure all the components of the vector field at the same time, thus, here we show only the scaling exponents obtained using magnetic field differences $B_{r}(t+\tau)-B_{r}(t)$ calculated from the radial component in a toroidal device where the $z$-axis is directed along the axis of the torus. As it can be seen, intermittency in magnetic turbulence is not so strong as appears in the solar wind, actually the degree of intermittency increases going toward the external wall. This last feature appears to be similar to what is currently observed in channel flows, where intermittency increases going towards the external wall (Pope, 2000).

Scaling exponents of structure functions for Alfvén variables, velocity, and magnetic variables

Living Reviews in Solar Physics

http: //www . livingreviews . org/lrsp-2005-4 
Table 2: Normalized scaling exponents $\xi_{p} / \xi_{3}$ for radial magnetic fluctuations in a laboratory plasma, as measured at different distances $r / R(R \simeq 0.45 \mathrm{~cm}$ being the minor radius of the torus in the experiment) from the external wall. Errors represent the standard deviations of the linear fitting. Scaling exponents have been obtained using the ESS.

\begin{tabular}{lllll}
\hline$p$ & $r / R=0.96$ & $r / R=0.93$ & $r / R=0.90$ & $r / R=0.86$ \\
\hline 1 & $0.39 \pm 0.01$ & $0.38 \pm 0.01$ & $0.37 \pm 0.01$ & $0.36 \pm 0.01$ \\
2 & $0.74 \pm 0.01$ & $0.73 \pm 0.02$ & $0.71 \pm 0.01$ & $0.70 \pm 0.01$ \\
3 & 1.00 & 1.00 & 1.00 & 1.00 \\
4 & $1.20 \pm 0.02$ & $1.24 \pm 0.02$ & $1.27 \pm 0.01$ & $1.28 \pm 0.01$ \\
5 & $1.32 \pm 0.03$ & $1.41 \pm 0.03$ & $1.51 \pm 0.03$ & $1.55 \pm 0.03$ \\
6 & $1.38 \pm 0.04$ & $1.50 \pm 0.04$ & $1.71 \pm 0.03$ & $1.78 \pm 0.04$ \\
\hline
\end{tabular}

Table 3: Normalized scaling exponents $\xi_{p} / \xi_{3}$ for Alfvénic, velocity, and magnetic fluctuations obtained from data of high resolution 2D MHD numerical simulations. Scaling exponents have been calculated from spatial fluctuations; different times, in the statistically stationary state, have been used to improve statistics. The scaling exponents have been calculated by ESS using Equation (27) as characteristic scale rather than the third-order structure function (cf. Politano et al., 1998b for details).

\begin{tabular}{lllll}
\hline$p$ & $Z^{+}$ & $Z^{-}$ & $v$ & $B$ \\
\hline 1 & $0.36 \pm 0.06$ & $0.56 \pm 0.06$ & $0.37 \pm 0.01$ & $0.46 \pm 0.02$ \\
2 & $0.70 \pm 0.05$ & $0.83 \pm 0.05$ & $0.70 \pm 0.01$ & $0.78 \pm 0.01$ \\
3 & 1.00 & 1.00 & 1.00 & 1.00 \\
4 & $1.28 \pm 0.02$ & $1.14 \pm 0.02$ & $1.28 \pm 0.02$ & $1.18 \pm 0.02$ \\
5 & $1.53 \pm 0.03$ & $1.25 \pm 0.03$ & $1.54 \pm 0.03$ & $1.31 \pm 0.03$ \\
6 & $1.79 \pm 0.05$ & $1.35 \pm 0.05$ & $1.78 \pm 0.05$ & $1.40 \pm 0.03$ \\
\hline
\end{tabular}

have been calculated also for high resolution 2D incompressible MHD numerical simulations Politano et al. (1998b). In this case, we are free from the Taylor hypothesis in calculating the fluctuations at a given scale. From 2D simulations we recover the fields $\mathbf{u}(\mathbf{r}, t)$ and $\mathbf{b}(\mathbf{r}, t)$ at some fixed times. We calculate the longitudinal fluctuations directly in space at a fixed time, namely $\delta u_{\ell}=[\mathbf{u}(\mathbf{r}+\ell, \mathbf{t})-\mathbf{u}(\mathbf{r}, \mathbf{t})] \cdot \ell / \ell$ (the same are made for different fields, namely the magnetic field or the Elsässer fields). Finally, averaging both in space and time, we calculate the scaling exponents through the structure functions. These scaling exponents are reported in Table 3. Note that, even in numerical simulations, intermittency for magnetic variables is stronger than for the velocity field.

\subsection{Probability density functions and self-similarity of fluctuations}

The presence of scaling laws for fluctuations is a signature of the presence of self-similarity in the phenomenon. A given observable $u(\ell)$, which depends on a scaling variable $\ell$, is invariant with respect to the scaling relation $\ell \rightarrow \lambda \ell$, when there exists a parameter $\mu(\lambda)$ such that $u(\ell)=$ $\mu(\lambda) u(\lambda \ell)$. The solution of this last relation is a power law $u(\ell)=C \ell^{h}$, where the scaling exponent is $h=-\log _{\lambda} \mu$.

Since, as we have just seen, turbulence is characterized by scaling laws, this must be a signature 
of self-similarity for fluctuations. Let us see what this means. Let us consider fluctuations at two different scales, namely $\delta z_{\ell}^{ \pm}$and $\delta z_{\lambda \ell}^{ \pm}$. Their ratio $\delta z_{\lambda \ell}^{ \pm} / \delta z_{\ell}^{ \pm} \sim \lambda^{h}$ depends only on the value of $h$, and this should imply that fluctuations are self-similar. This means that PDFs are related through

$$
P\left(\delta z_{\lambda \ell}^{ \pm}\right)=P D F\left(\lambda^{h} \delta z_{\ell}^{ \pm}\right) .
$$

Let us consider the standardized variables

$$
y_{\ell}^{ \pm}=\frac{\delta z_{\ell}^{ \pm}}{\left\langle\left(\delta z_{\ell}^{ \pm}\right)^{2}\right\rangle^{1 / 2}} .
$$

It can be easily shown that when $h$ is unique or in a pure self-similar situation, PDFs are related through $P\left(y_{\ell}^{ \pm}\right)=P D F\left(y_{\lambda \ell}^{ \pm}\right)$, say by changing scale PDFs coincide.

The PDFs relative to the standardized magnetic fluctuations $\Delta b_{r}=\delta b_{r} /\left\langle\delta b_{r}^{2}\right\rangle^{1 / 2}$, at three different scales $r$, are shown in Figure 74. It appears evident that the global self-similarity in real turbulence is broken. PDFs do not coincide at different scales, rather their shape seems to depend on the scale $r$. In particular, at large scales PDFs seem to be almost Gaussian, but they become more and more stretched as $r$ decreases. At the smallest scale PDFs are stretched exponentials. This scaling dependence of PDFs is a different way to say that scaling exponents of fluctuations are anomalous, or can be taken as a different definition of intermittency. Note that the wings of PDFs are higher than those of a Gaussian function. This implies that intense fluctuations have a probability of occurrence higher than that they should have if they were Gaussianly distributed. Said differently, intense stochastic fluctuations are less rare than we should expect from the point of view of a Gaussian approach to the statistics. These fluctuations play a key role in the statistics of turbulence. The same statistical behavior can be found in different experiments related to the study of the atmosphere (see Figure 75) and the laboratory plasma (see Figure 76).

\subsection{What is intermittent in the solar wind turbulence? The multifractal approach}

Time dependence of $\delta u_{\tau}$ and $\delta b_{\tau}$ for three different scales $\tau$ is shown in Figures 77 and 78, respectively. These plots show that, as $\tau$ becomes small, intense fluctuations become more and more important, and they dominate the statistics. Fluctuations at large scales appear to be smooth while, as the scale becomes smaller, intense fluctuations becomes visible. These dominating fluctuations represent relatively rare events. Actually, at the smallest scales, the time behavior of both $\delta u_{\tau}$ and $\delta b_{\tau}$ is dominated by regions where fluctuations are low, in between regions where fluctuations are intense and turbulent activity is very high. Of course, this behavior cannot be described by a global self-similar behavior. It appears more convincing a description where scaling laws must depend on the region of turbulence we are investigating. The behavior we have just described is at the heart of the multifractal approach to turbulence (Frisch, 1995). In that description of turbulence, even if the small scales of fluid flow cannot be globally self-similar, self-similarity can be reintroduced as a local property. In the multifractal description it is conjectured that turbulent flows can be made by an infinite set of points $S_{h}(\mathbf{r})$, each set being characterized by a scaling law $\delta Z_{\ell}^{ \pm} \sim \ell^{h(\mathbf{r})}$, that is, the scaling exponent can depend on the position $\mathbf{r}$. The usual dimension of that set is then not constant, but depends on the local value of $h$, and is quoted as $D(h)$ in literature. Then, the probability of occurrence of a given fluctuation can be calculated through the weight the fluctuation assumes within the whole flow, i.e.,

$$
P\left(\delta Z_{\ell}^{ \pm}\right) \sim\left(\delta Z_{\ell}^{ \pm}\right)^{h} \times \text { Volume occupied by fluctuations }
$$

Living Reviews in Solar Physics

http: //www . livingreviews . org/lrsp-2005-4 


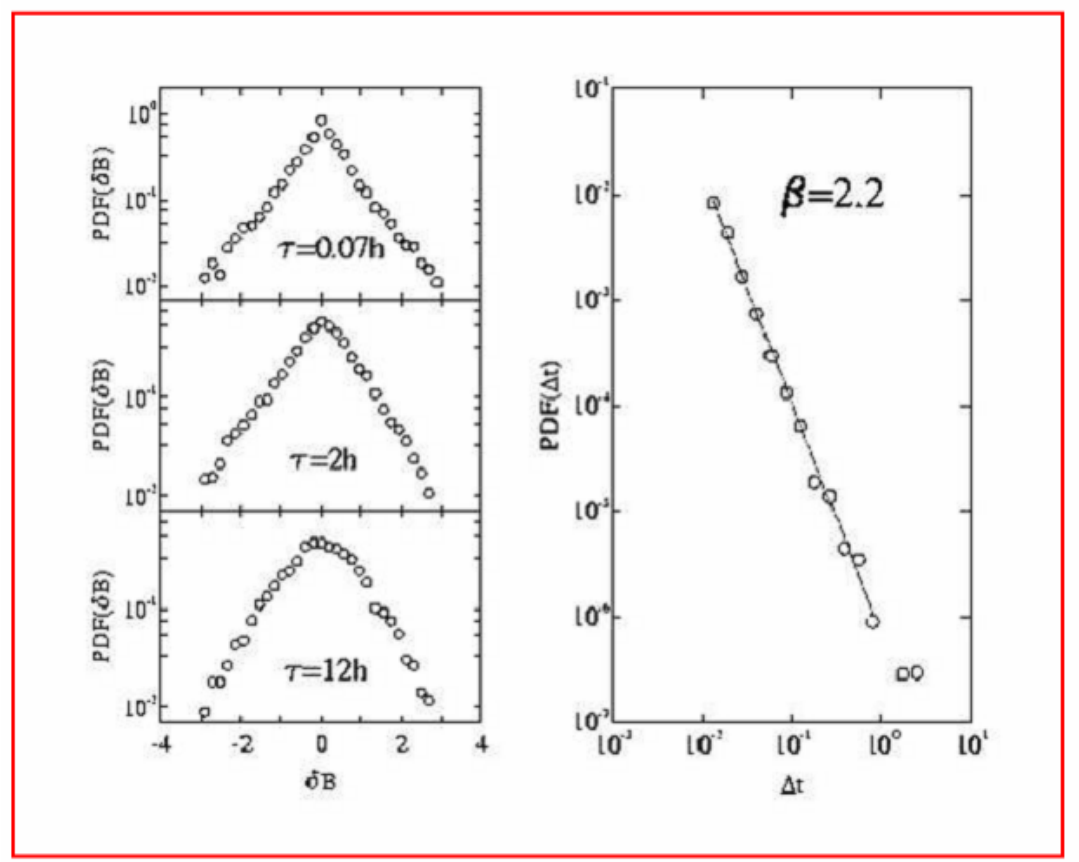

Figure 74: Left column: normalized PDFs for the magnetic fluctuations observed in the solar wind turbulence. Right panel: distribution function of waiting times $\Delta t$ between structures at the smallest scale. The parameter $\beta$ is the scaling exponent of the scaling relation $P D F(\Delta t) \sim \Delta t^{-\beta}$ for the distribution function of waiting times. 


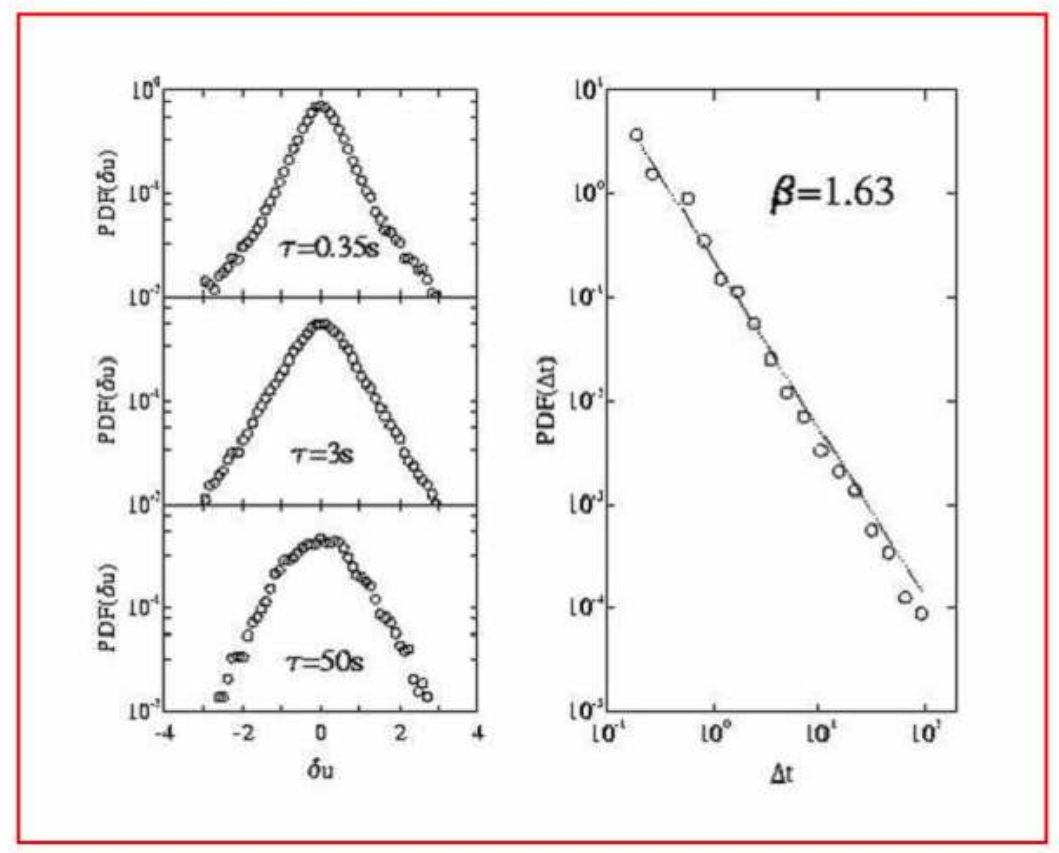

Figure 75: Left column: normalized PDFs of velocity fluctuations in atmospheric turbulence. Right panel: distribution function of waiting times $\Delta t$ between structures at the smallest scale. The parameter $\beta$ is the scaling exponent of the scaling relation $P D F(\Delta t) \sim \Delta t^{-\beta}$ for the distribution function of waiting times. The turbulent samples have been collected above a grass-covered forest clearing at $5 \mathrm{~m}$ above the ground surface and at a sampling rate of $56 \mathrm{~Hz}$ (Katul et al., 1997).

Living Reviews in Solar Physics

http://www. livingreviews.org/lrsp-2005-4 


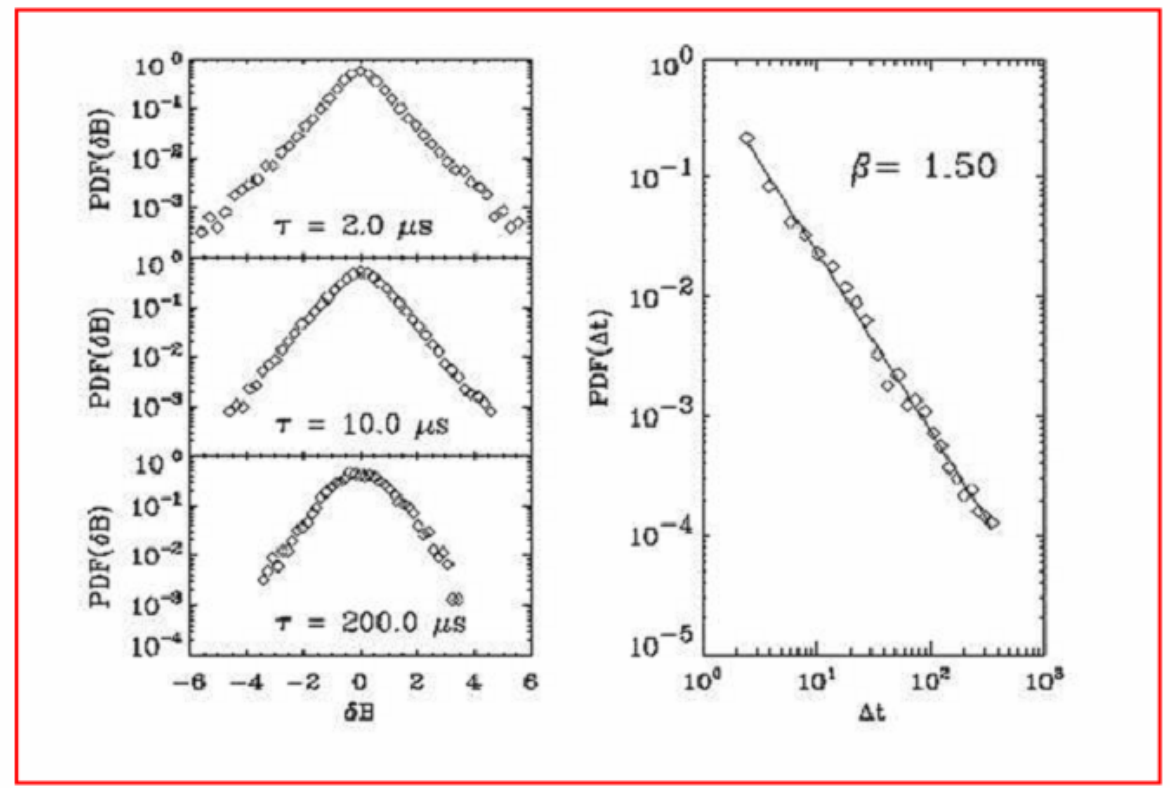

Figure 76: Left column: normalized PDFs of the radial magnetic field collected in RFX magnetic turbulence (Carbone et al., 2000). Right panel: distribution function of waiting times $\Delta t$ between structures at the smallest scale. The parameter $\beta$ is the scaling exponent of the scaling relation $\operatorname{PDF}(\Delta t) \sim \Delta t^{-\beta}$ for the distribution function of waiting times.

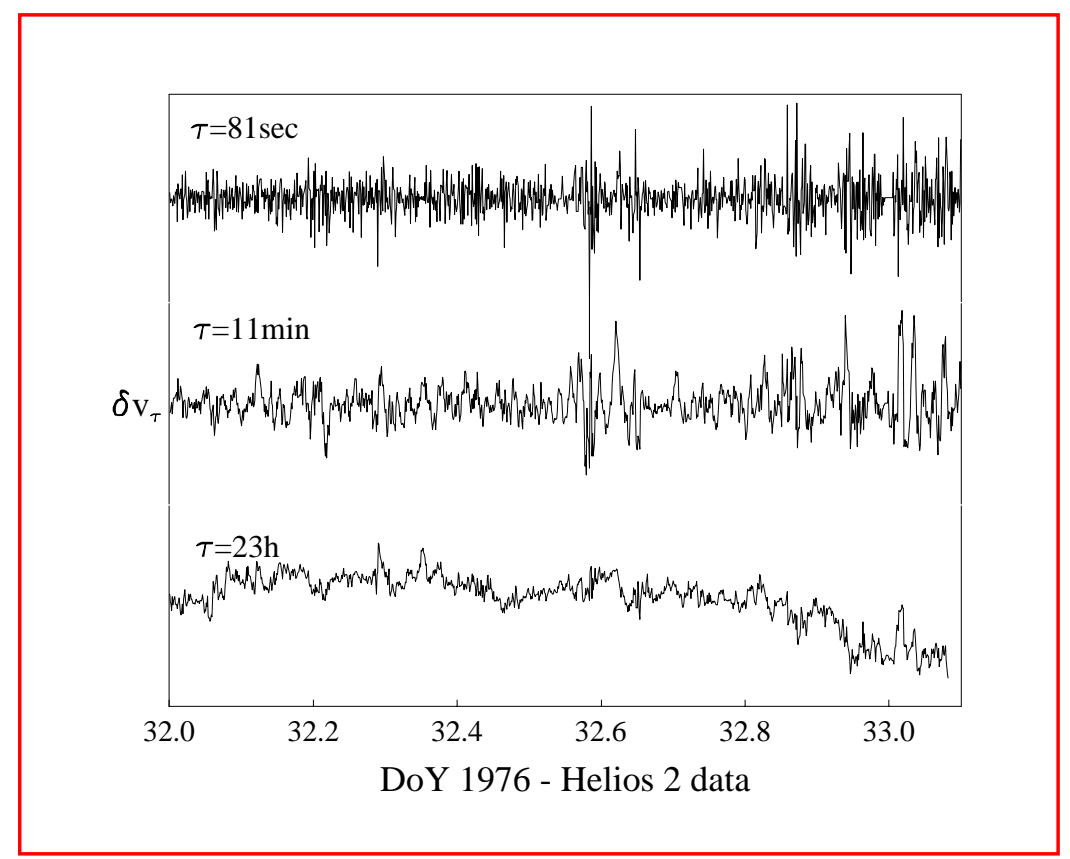

Figure 77: Differences for the longitudinal velocity $\delta u_{\tau}=u(t+\tau)-u(t)$ at three different scales $\tau$, as shown in the figure. 


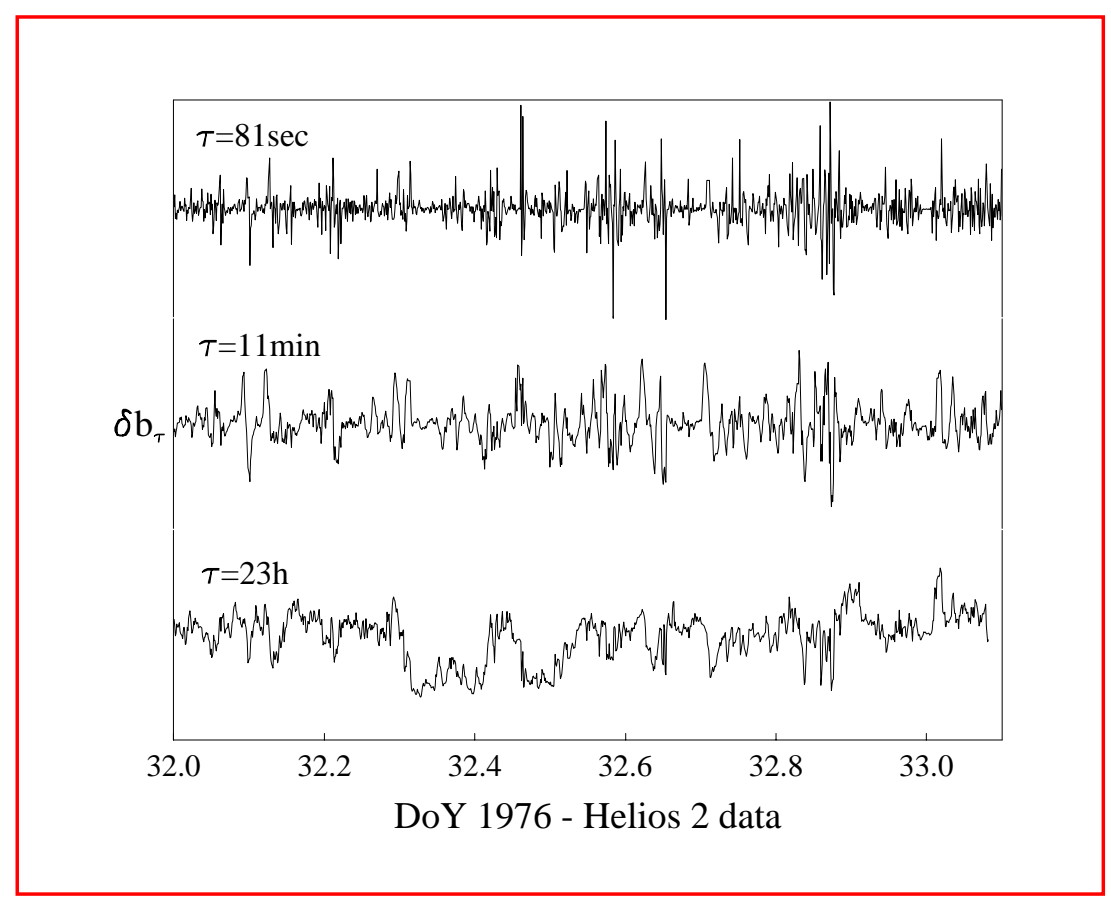

Figure 78: Differences for the magnetic intensity $\delta b_{\tau}=B(t+\tau)-B(t)$ at three different scales $\tau$, as shown in the figure.

and the $p$-th order structure function is immediately written through the integral over all (continuous) values of $h$ weighted by a smooth function $\mu(h) \sim 0(1)$, i.e.,

$$
S_{p}(\ell)=\int \mu(h)\left(\delta Z_{\ell}^{ \pm}\right)^{p h}\left(\delta Z_{\ell}^{ \pm}\right)^{3-D(h)} d h .
$$

A moment of reflection allows us to realize that in the limit $\ell \rightarrow 0$ the integral is dominated by the minimum value (over $h$ ) of the exponent and, as shown by Frisch (1995), the integral can be formally solved using the usual saddle-point method. The scaling exponents of the structure function can then be written as

$$
\zeta_{p}=\min _{h}[p h+3-D(h)] .
$$

In this way, the departure of $\zeta_{p}$ from the linear Kolmogorov scaling and then intermittency, can be characterized by the continuous changing of $D(h)$ as $h$ varies. That is, as $p$ varies we are probing regions of fluid where even more rare and intense events exist. These regions are characterized by small values of $h$, that is, by stronger singularities of the gradient of the field.

Owing to the famous Landau footnote on the fact that fluctuations of the energy transfer rate must be taken into account in determining the statistics of turbulence, people tried to interpret the non-linear energy cascade typical of turbulence theory, within a geometrical framework. The old Richardson's picture of the turbulent behavior as the result of a hierarchy of eddies at different scales has been modified and, as realized by Kraichnan (1974), once we leave the idea of a constant energy cascade rate we open a "Pandora's box" of possibilities for modeling the energy cascade. By looking at scaling laws for $\delta z_{\ell}^{ \pm}$and introducing the scaling exponents for the energy transfer rate $\left\langle\epsilon_{\ell}^{p}\right\rangle \sim r^{\tau_{p}}$, it can be found that $\zeta_{p}=p / m+\tau_{p / m}$ (being $m=3$ when the Kolmogorov-like phenomenology is taken into account, or $m=4$ when the fluid is magnetically dominated). In

Living Reviews in Solar Physics

http: //www . livingreviews . org/lrsp-2005-4 
this way the intermittency correction are determined by a cascade model for the energy transfer rate. When $\tau_{p}$ is a non-linear function of $p$, the energy transfer rate can be described within the multifractal geometry (see, e.g., Meneveau, 1991 and references therein) characterized by the generalized dimensions $D_{p}=1-\tau_{p} /(p-1)$ (Hentschel and Procaccia, 1983). The scaling exponents of the structure functions are then related to $D_{p}$ by

$$
\zeta_{p}=\left(\frac{p}{m}-1\right) D_{p / m}+1
$$

The correction to the linear scaling $p / m$ is positive for $p<m$, negative for $p>m$, and zero for $p=m$. A fractal behavior where $D_{p}=$ const. $<1$ gives a linear correction with a slope different from $1 / m$.

\subsection{Fragmentation models for the energy transfer rate}

Cascade models can be organized as a collection of fragments at a given scale $\ell$, which results from the fragmentation of structures at the scale $\ell^{\prime}>\ell$, down to the dissipative scale (Novikov, 1969). Sophisticated statistics are applied to obtain scaling exponents $\zeta_{p}$ for the $p$-th order structure function.

The random- $\beta$ model (Benzi et al., 1984) can be derived by invoking that the space-filling factor for the fragments at a given scale in the energy cascade is given by a random variable $\beta$. The probability of occurrence of a given $\beta$ is assumed to be a bimodal distribution where the eddies fragmentation process generates either space-filling eddies with probability $\xi$ or planar sheets with probability $(1-\xi)$ (for conservation $0 \leq \xi \leq 1$ ). It can be found that

$$
\zeta_{p}=\frac{p}{m}-\log _{2}\left[1-\xi+\xi 2^{p / m-1}\right],
$$

where the free parameter $\xi$ can be fixed through a fit on the data.

The $p$-model (Meneveau, 1991; Carbone, 1993) consists in an eddies fragmentation process described by a two-scale Cantor set with equal partition intervals. An eddy at the scale $\ell$, with an energy derived from the transfer rate $\epsilon_{r}$, breaks down into two eddies at the scale $\ell / 2$, with energies $\mu \epsilon_{r}$ and $(1-\mu) \epsilon_{r}$. The parameter $0.5 \leq \mu \leq 1$ is not defined by the model, but is fixed from the experimental data. The model gives

$$
\zeta_{p}=1-\log _{2}\left[\mu^{p / m}+(1-\mu)^{p / m}\right] .
$$

In the model by She and Leveque (see, e.g., She and Leveque, 1994; Politano and Pouquet, 1998) one assumes an infinite hierarchy for the moments of the energy transfer rates, leading to $\epsilon_{r}^{(p+1)} \sim\left[\epsilon_{r}^{(p)}\right]^{\beta}\left[\epsilon_{r}^{(\infty)}\right]^{1-\beta}$, and a divergent scaling law for the infinite-order moment $\epsilon_{r}^{(\infty)} \sim r^{-x}$, which describes the most singular structures within the flow. The model reads

$$
\zeta_{p}=\frac{p}{m}(1-x)+\mathrm{C}\left[1-\left(1-\frac{x}{\mathrm{C}}\right)^{p / m}\right] .
$$

The parameter $\mathrm{C}=x /(1-\beta)$ is identified as the codimension of the most singular structures. In the standard MHD case (Politano and Pouquet, 1995) $x=\beta=1 / 2$, so that $\mathrm{C}=1$, that is, the most singular dissipative structures are planar sheets. On the contrary, in fluid flows $\mathrm{C}=2$ and the most dissipative structures are filaments. The large $p$ behavior of the $p$-model is given by $\zeta_{p} \sim(p / m) \log _{2}(1 / \mu)+1$, so that Equations $(40,41)$ give the same results providing $\mu \simeq 2^{-x}$. As shown by Carbone et al. (1996b) models are able to capture intermittency of fluctuations in the solar wind. The agreement between the curves $\zeta_{p}$ and normalized scaling exponents is excellent, and this means that we realistically cannot discriminate between the models we reported above. 


\subsection{A model for the departure from self-similarity}

Besides the idea of self-similarity underlying the process of energy cascade in turbulence, a different point of view can be introduced. That is a model that tries to characterize the behavior of the PDFs through the scaling laws of the parameters, which describe how the shape of the PDFs changes going towards small scales. In its simplest form the model can be introduced by saying that PDFs of increments $\delta Z_{\ell}^{ \pm}$, at a given scale, are built up by a convolution of Gaussian distributions of width $\sigma=\left\langle\left(\delta Z_{\ell}^{ \pm}\right)^{2}\right\rangle^{1 / 2}$, whose distribution is given by $G_{\lambda}(\sigma)$, namely

$$
P\left(\delta Z_{\ell}^{ \pm}\right)=\frac{1}{2 \pi} \int_{0}^{\infty} G_{\lambda}(\sigma) \exp \left(-\frac{\left(\delta Z_{\ell}^{ \pm}\right)^{2}}{2 \sigma^{2}}\right) \frac{d \sigma}{\sigma}
$$

In a purely self-similar situation, where the energy cascade generates only a trivial variation of $\sigma$ with scales, the width of the distribution $G_{\lambda}(\sigma)$ is zero and, invariably, we recover a Gaussian distribution for $P\left(\delta Z_{\ell}^{ \pm}\right)$. On the contrary, when the cascade is not strictly self-similar, the width of $G_{\lambda}(\sigma)$ is different from zero and the scaling behavior of the width $\lambda^{2}$ of $G_{\lambda}(\sigma)$ can be used to characterize intermittency.

\subsection{Intermittency properties recovered via a shell model}

The FSGC shell model has remarkable properties which closely resemble those typical of MHD phenomena (Giuliani and Carbone, 1998). However, the presence of a constant forcing term always induces a dynamical alignment, unless the model is forced appropriately, which invariably brings the system towards a state in which velocity and magnetic fields are strongly correlated, that is, where $Z_{n}^{ \pm} \neq 0$ and $Z_{n}^{\mp}=0$. When we want to compare statistical properties of turbulence described by MHD shell models with solar wind observations, this term should be avoided. It is possible to replace the constant forcing term by an exponentially time-correlated Gaussian random forcing which is able to destabilize the Alfvénic fixed point of the model (Giuliani and Carbone, 1998), thus assuring the energy cascade. The forcing is obtained by solving the following Langevin equation:

$$
\frac{d F_{n}}{d t}=-\frac{F_{n}}{\tau}+\mu(t)
$$

where $\mu(t)$ is a Gaussian stochastic process $\delta$-correlated in time $\left\langle\mu(t) \mu\left(t^{\prime}\right)\right\rangle=2 D \delta\left(t^{\prime}-t\right)$. This kind of forcing will be used to investigate statistical properties.

A statistically stationary state is reached by the system, as shown in Giuliani and Carbone (1998), with a well defined inertial range, say a region where Equation (29) is verified. Spectra for both the velocity $\left|u_{n}(t)\right|^{2}$ and magnetic $\left|b_{n}(t)\right|^{2}$ variables, as a function of $k_{n}$, obtained in the stationary state, are shown in Figures 79 and 80. Fluctuations are averaged over time. The Kolmogorov spectrum is also reported as a solid line. It is worthwhile to remark that, by adding a random term like $i k_{n} B_{0}(t) Z_{n}^{ \pm}$to a little modified version of the MHD GOY shell model $\left(B_{0}\right.$ is a random function with some statistical characteristics), a Kraichnan spectrum, say $E\left(k_{n}\right) \sim k_{n}^{-3 / 2}$, where $E\left(k_{n}\right)$ is the total energy, can be recovered (Hattori and Ishizawa, 2001). The term added to the model could represent the effect of the occurrence of a large-scale magnetic field.

Intermittency in the shell model is due to the time behavior of shell variables. It has been shown (Okkels, 1997) that the evolution of GOY model consists of short bursts traveling through the shells and long period of oscillations before the next burst arises. In Figures 81 and 82 we report the time evolution of the real part of both velocity variables $u_{n}(t)$ and magnetic variables $b_{n}(t)$ at three different shells. It can be seen that, while at smaller $k_{n}$ variables seems to be Gaussian, at larger $k_{n}$ variables present very sharp fluctuations in between very low fluctuations.

The time behavior of variables at different shells changes the statistics of fluctuations. In Figure 83 we report the probability density functions $P\left(\delta u_{n}\right)$ and $P\left(\delta B_{n}\right)$, for different shells $n$,

Living Reviews in Solar Physics

http: //www. livingreviews . org/lrsp-2005-4 


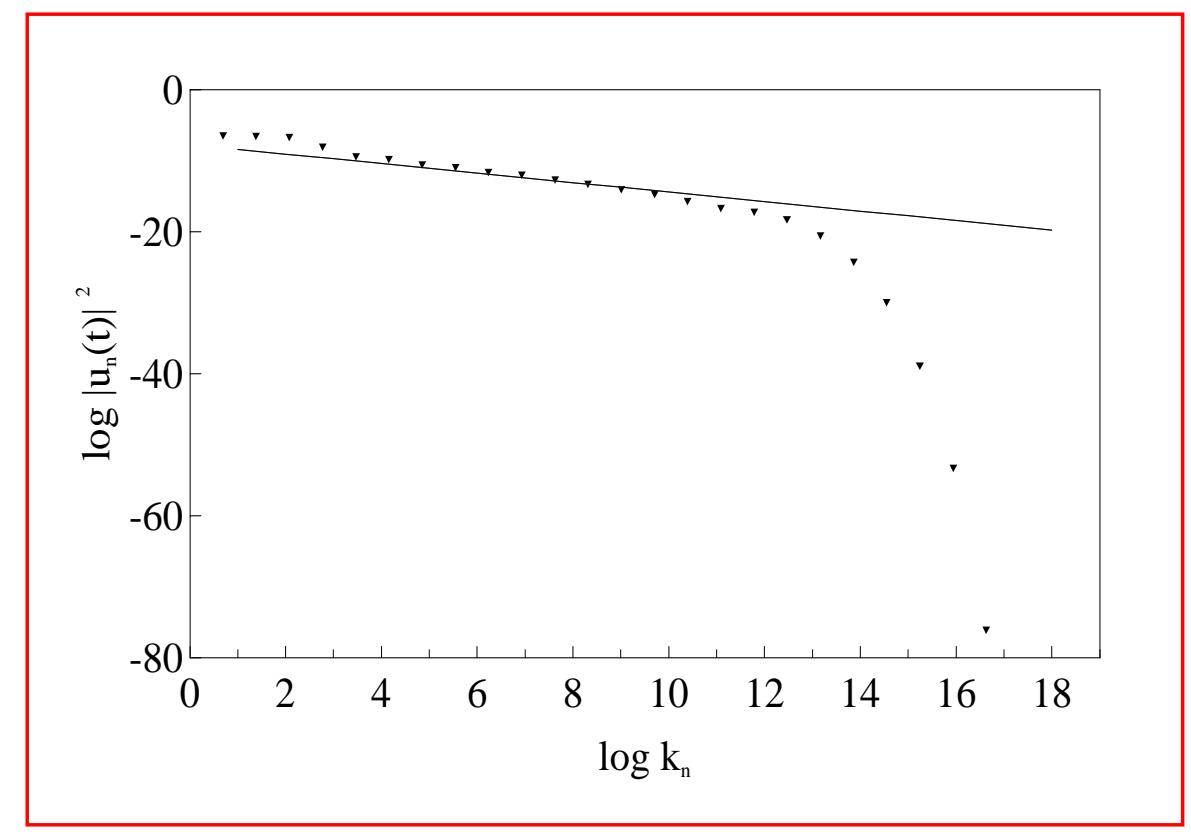

Figure 79: We show the kinetic energy spectrum $\left|u_{n}(t)\right|^{2}$ as a function of $\log _{2} k_{n}$ for the MHD shell model. The full line refer to the Kolmogorov spectrum $k_{n}^{-2 / 3}$.

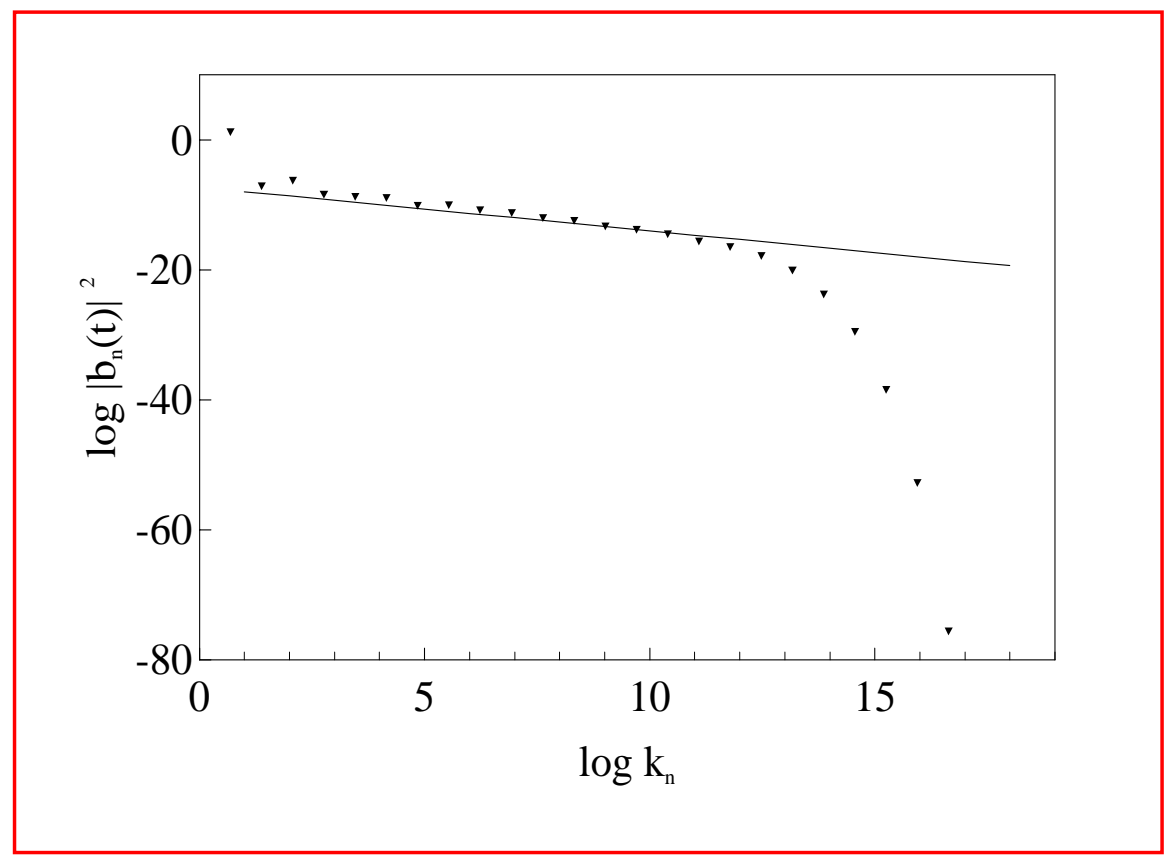

Figure 80: We show the magnetic energy spectrum $\left|b_{n}(t)\right|^{2}$ as a function of $\log _{2} k_{n}$ for the MHD shell model. The full line refer to the Kolmogorov spectrum $k_{n}^{-2 / 3}$. 


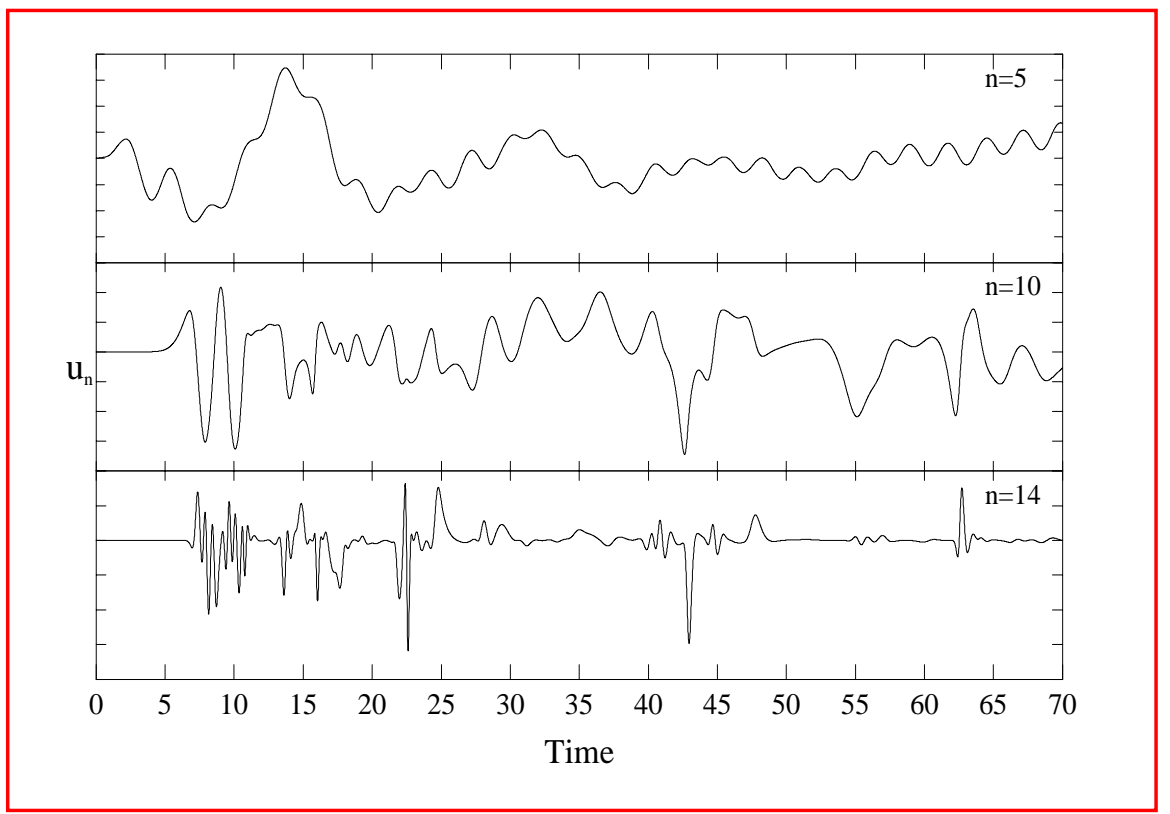

Figure 81: Time behavior of the real part of velocity variable $u_{n}(t)$ at three different shells $n$, as indicated in the different panels.

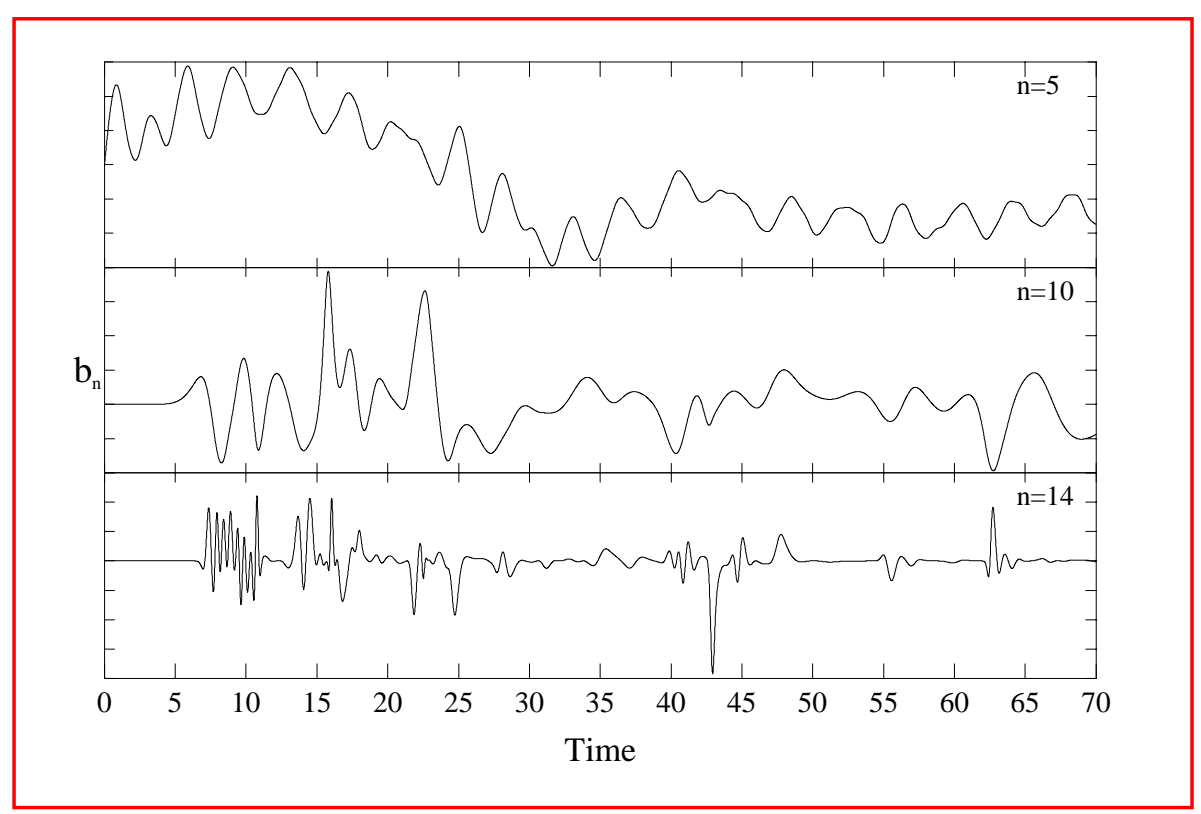

Figure 82: Time behavior of the real part of magnetic variable $b_{n}(t)$ at three different shells $n$, as indicated in the different panels.

Living Reviews in Solar Physics

http://www . livingreviews . org/lrsp-2005-4 
of standardized variables

$$
\delta u_{n}=\frac{\Re e\left(u_{n}\right)}{\sqrt{\left\langle\left|u_{n}\right|^{2}\right\rangle}} \quad \text { and } \quad \delta B_{n}=\frac{\Re e\left(b_{n}\right)}{\sqrt{\left\langle\left|b_{n}\right|^{2}\right\rangle}},
$$

where $\Re e$ indicates that we take the real part of $u_{n}$ and $b_{n}$. Typically we see that PDFs look differently at different shells: At small $k_{n}$ fluctuations are quite Gaussian distributed, while at large $k_{n}$ they tend to become increasingly non-Gaussian, by developing fat tails. Rare fluctuations have a probability of occurrence larger than a Gaussian distribution. This is the typical behavior of intermittency as observed in usual fluid flows and described in previous sections.

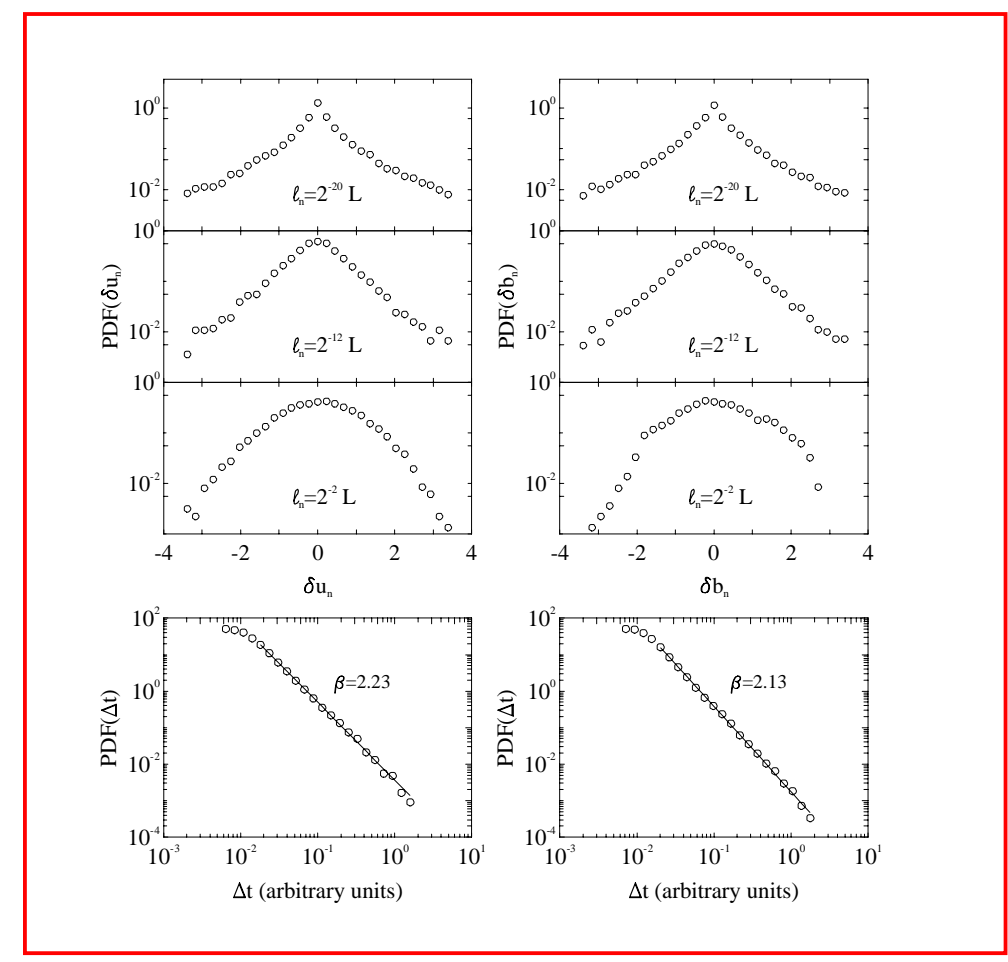

Figure 83: In the first three panels we report PDFs of both velocity (left column) and magnetic (right column) shell variables, at three different shells $\ell_{n}$. The bottom panels refer to probability distribution functions of waiting times between intermittent structures at the shell $n=12$ for the corresponding velocity and magnetic variables.

The same phenomenon gives rise to the departure of scaling laws of structure functions from a Kolmogorov scaling. Within the framework of the shell model the analogous of structure functions are defined as

$$
\left\langle\left|u_{n}\right|^{p}\right\rangle \sim k_{n}^{-\xi_{p}} ; \quad\left\langle\left|b_{n}\right|^{p}\right\rangle \sim k_{n}^{-\eta_{p}} ; \quad\left\langle\left|Z_{n}^{ \pm}\right|^{p}\right\rangle \sim k_{n}^{-\xi_{p}^{ \pm}}
$$

For MHD turbulence it is also useful to report mixed correlators of the flux variables, i.e.,

$$
\left\langle\left[T_{n}^{ \pm}\right]^{p / 3}\right\rangle \sim k_{n}^{-\beta_{p}^{ \pm}} .
$$

Scaling exponents have been determined from a least square fit in the inertial range $3 \leq n \leq 12$. The values of these exponents are reported in Table 4. It is interesting to notice that, while scaling 
Table 4: Scaling exponents for velocity and magnetic variables, Elsässer variables, and fluxes. Errors on $\beta_{p}^{ \pm}$are about one order of magnitude smaller than the errors shown.

\begin{tabular}{ccccccc}
\hline$p$ & $\zeta_{p}$ & $\eta_{p}$ & $\xi_{p}^{+}$ & $\xi_{p}^{-}$ & $\beta_{p}^{+}$ & $\beta_{p}^{-}$ \\
\hline 1 & $0.36 \pm 0.01$ & $0.35 \pm 0.01$ & $0.35 \pm 0.01$ & $0.36 \pm 0.01$ & 0.326 & 0.318 \\
2 & $0.71 \pm 0.02$ & $0.69 \pm 0.03$ & $0.70 \pm 0.02$ & $0.70 \pm 0.03$ & 0.671 & 0.666 \\
3 & $1.03 \pm 0.03$ & $1.01 \pm 0.04$ & $1.02 \pm 0.04$ & $1.02 \pm 0.04$ & 1.000 & 1.000 \\
4 & $1.31 \pm 0.05$ & $1.31 \pm 0.06$ & $1.30 \pm 0.05$ & $1.32 \pm 0.06$ & 1.317 & 1.323 \\
5 & $1.57 \pm 0.07$ & $1.58 \pm 0.08$ & $1.54 \pm 0.07$ & $1.60 \pm 0.08$ & 1.621 & 1.635 \\
6 & $1.80 \pm 0.08$ & $1.8 \pm 0.10$ & $1.79 \pm 0.09$ & $1.87 \pm 0.10$ & 1.91 & 1.94 \\
\hline
\end{tabular}

exponents for velocity are the same as those found in the solar wind, scaling exponents for the magnetic field found in the solar wind reveal a more intermittent character. Moreover, we notice that velocity, magnetic and Elsässer variables are more intermittent than the mixed correlators and we think that this could be due to the cancellation effects among the different terms defining the mixed correlators.

Time intermittency in the shell model generates rare and intense events. These events are the result of the chaotic dynamics in the phase-space typical of the shell model (Okkels, 1997). That dynamics is characterized by a certain amount of memory, as can be seen through the statistics of waiting times between these events. The distributions $P(\delta t)$ of waiting times is reported in the bottom panels of Figures 83, at a given shell $n=12$. The same statistical law is observed for the bursts of total dissipation (Boffetta et al., 1999).

Living Reviews in Solar Physics

http: //www . livingreviews . org/lrsp-2005-4 


\section{Intermittency Properties in the 3D Heliosphere: Taking a Look at the Data}

In this chapter, we present a reasoned look at the main aspect of what has been reported in literature about the problem of intermittency in the solar wind turbulence. In particular, we present results from data analysis.

\subsection{Structure functions}

Apart from the earliest investigations on the fractal structure of magnetic field as observed in interplanetary space (Burlaga and Klein, 1986), the starting point for the investigation of intermittency in the solar wind dates back to 1991, when Burlaga (1991a) started to look at the scaling of the bulk velocity fluctuations at $8.5 \mathrm{AU}$ using Voyager 2 data. This author found that anomalous scaling laws for structure functions could be recovered in the range $0.85 \leq r \leq 13.6 \mathrm{~h}$. This range of scales has been arbitrarily identified as a kind of "inertial range", say a region were a linear scaling exists between $\log S_{r}^{(p)}$ and $\log r$, and the scaling exponents have been calculated as the slope of these curves. However, structure functions of order $p \leq 20$ were determined on the basis of only about 4500 data points. Nevertheless the scaling was found to be quite in agreement with that found in ordinary fluid flows. Although the data might be in agreement with the random- $\beta$ model, from a theoretical point of view Carbone $(1993,1994 b)$ showed that normalized scaling exponents $\zeta_{p} / \zeta_{4}$ calculated by Burlaga (1991a) would be better fitted by using a $p$-model derived from the Kraichnan phenomenology (Kraichnan, 1965; Carbone, 1993), and considering the parameter $\mu \simeq 0.77$. The same author (Burlaga, 1991b) investigated the multifractal structure of the interplanetary magnetic field near $25 \mathrm{AU}$ and analyzed positive defined fields as magnetic field strength, temperature, and density using the multifractal machinery of dissipation fields (Paladin and Vulpiani, 1987; Meneveau, 1991). Burlaga (1991c) showed that intermittent events observed in corotating streams at $1 \mathrm{AU}$ should be described by a multifractal geometry. Even in this case the number of points used was very low to assure the reliability of high-order moments.

Marsch and Liu (1993) investigated the structure of intermittency of the turbulence observed in the inner heliosphere by using Helios 2 data. They analyzed both bulk velocity and Alfvén speed to calculate structure functions in the whole range $40.5 \mathrm{~s}$ (the instrument resolution) up to $24 \mathrm{~h}$ to estimate the $p$-th order scaling exponents. Note that also in this analysis the number of data points used was too small to assure a reliability for order $p=20$ structure functions as reported by Marsch and Liu (1993). From the analysis analogous to Burlaga (1991a), authors found that anomalous scaling laws are present. A comparison between fast and slow streams at two heliocentric distances, namely $0.3 \mathrm{AU}$ and $1 \mathrm{AU}$, allows authors to conjecture a scenario for high speed streams were Alfvénic turbulence, originally self-similar (or poorly intermittent) near the Sun, "... loses its self-similarity and becomes more multifractal in nature" (Marsch and Liu, 1993), which means that intermittent corrections increase from 0.3 AU to 1 AU. No such behavior seems to occur in the slow solar wind. From a phenomenological point of view, Marsch and Liu (1993) found that data can be fitted with a piecewise linear function for the scaling exponents $\zeta_{p}$, namely a $\beta$-model $\zeta_{p}=3-D+p(D-2) / 3$, where $D \simeq 3$ for $p \leq 6$ and $D \simeq 2.6$ for $p>6$. Authors say that "We believe that we see similar indications in the data by Burlaga, who still prefers to fit his whole $\zeta_{p}$ dataset with a single fit according to the non-linear random $\beta$-model.". We like to comment that the impression by Marsch and Liu (1993) is due to the fact that the number of data points used was very small. As a matter of fact, only structure functions of order $p \leq 4$ are reliably described by the number of points used by Burlaga (1991a).

However, the data analyses quoted above, which in some sense present some contradictory results, are based on high order statistics which is not supported by an adequate number of data points and the range of scales, where scaling laws have been recovered, is not easily identifiable. 
To overcome these difficulties Carbone et al. (1996a) investigated the behavior of the normalized ratios $\zeta_{p} / \zeta_{3}$ through the ESS procedure described above, using data coming from low-speed streams measurements of Helios 2 spacecraft. Using ESS the whole range covered by measurements is linear, and scaling exponent ratios can be reliably calculated. Moreover, to have a dataset with a high number of points, authors mixed in the same statistics data coming from different heliocentric distances (from $0.3 \mathrm{AU}$ up to $1 \mathrm{AU}$ ). This is not correct as far as fast wind fluctuations are taken into account, because, as found by Marsch and Liu (1993) and Bruno et al. (2003b), there is a radial evolution of intermittency. Results showed that intermittency is a real characteristic of turbulence in the solar wind, and that the curve $\zeta_{p} / \zeta_{3}$ is a non-linear function of $p$ as soon as values of $p \leq 6$ are considered.

Marsch et al. (1996) for the first time investigated the geometrical and scaling properties of the energy flux along the turbulent cascade and dissipation rate of kinetic energy. They showed the multifractal nature of the dissipation field and estimated, for the first time in solar wind MHD turbulence, the associated singularity spectrum which resulted to be very similar to those obtained for ordinary fluid turbulence (Meneveau and Sreenivasan, 1987b). They also estimated the energy dissipation rate for time scales of $10^{2} \mathrm{~s}$ to be around $5.4 \times 10^{-16} \mathrm{erg} \mathrm{cm}^{-3} \mathrm{~s}^{-1}$. This value was similar to the theoretical heating rate required in the model by Tu (1988) with Alfvén waves to explain the radial temperature dependence observed in fast solar wind.

Looking at the literature, it can be realized that often scaling exponents $\zeta_{p}$, as observed mainly in the high-speed streams of the inner solar wind, cannot be explained properly by any cascade model for turbulence. This feature has been attributed to the fact that this kind of turbulence is not in a fully-developed state with a well defined spectral index. Models developed by Tu et al. (1984) and Tu (1988) were successful in describing the evolution of the observed power spectra. Using the same idea Tu et al. (1996) and Marsch and Tu (1997) investigated the behavior of an extended cascade model developed on the base of the $p$-model (Meneveau and Sreenivasan, 1987a; Carbone, 1993). Authors conjectured that: i) the scaling laws for fluctuations are still valid in the form $\delta Z_{\ell}^{ \pm} \sim \ell^{h}$, even when turbulence is not fully developed; ii) the energy cascade rate is not constant, its moments rather depend not only on the generalized dimensions $D_{p}$ but also on the spectral index $\alpha$ of the power spectrum, say $\left\langle\epsilon_{r}^{p}\right\rangle \sim \epsilon^{p}(\ell, \alpha) \ell^{(p-1) D_{p}}$, where the averaged energy transfer rate is assumed to be

$$
\epsilon(\ell, \alpha) \sim \ell^{-(m / 2+1)} P_{\ell}^{\alpha / 2},
$$

being $P_{\ell} \sim \ell^{\alpha}$ the usual energy spectrum $(\ell \sim 1 / k)$. The model gives

$$
\zeta_{p}=1+\left(\frac{p}{m}-1\right) D_{p / m}+\left[\alpha \frac{m}{2}-\left(1+\frac{m}{2}\right)\right] \frac{p}{m},
$$

where the generalized dimensions are recovered from the usual $p$-model

$$
D_{p}=\frac{\log _{2}\left[\mu^{p}+(1-\mu)^{p}\right]}{(1-p)} .
$$

In the limit of "fully developed turbulence", say when the spectral slope is $\alpha=2 / m+1$ the usual Equation (40) is recovered. The Helios 2 data are consistent with this model as far as the parameters are $\mu \simeq 0.77$ and $\alpha \simeq 1.45$, and the fit is relatively good (Tu et al., 1996). Recently, Horbury et al. (1997) and Horbury and Balogh (1997) studied the magnetic field fluctuations of the polar high-speed turbulence from Ulysses measurements at $3.1 \mathrm{AU}$ and at $63^{\circ}$ heliolatitude. These authors showed that the observed magnetic field fluctuations were in agreement with the intermittent turbulence p-model of Meneveau and Sreenivasan (1987a). They also showed that the scaling exponents of structure functions of order $p \leq 6$, in the scaling range $20 \leq r \leq 300 \mathrm{~s}$ followed the Kolmogorov scaling instead of Kraichnan scaling as expected. In addition, the same authors (Horbury et al., 1997) estimated the applicability of the model by Tu et al. (1996) and

Living Reviews in Solar Physics

http: //www . livingreviews . org/lrsp-2005-4 
Marsch and Tu (1997) to the spectral transition range where the spectral index changes during the spectral evolution and concluded that this model was able to fit the observations much better than the $p$-model when values of the parameters $p$ change continuously with the scale.

Analysis of scaling exponents of $p$-th order structure functions has been performed using different spacecraft datasets of Ulysses spacecraft. Horbury et al. (1995a) and Horbury et al. (1995c) investigated the structure functions of magnetic field as obtained from observations recorded between 1.7 and $4 \mathrm{AU}$, and covering a heliographic latitude between $40^{\circ}$ and $80^{\circ}$ south. By investigating the spectral index of the second order structure function, they found a decrease with heliocentric distance attributed to the radial evolution of fluctuations. Further investigations (see, e.g., Ruzmaikin et al., 1995) were obtained using structure functions to study the Ulysses magnetic field data in the range of scales $1 \leq r \leq 32 \mathrm{~min}$. Ruzmaikin et al. (1995) showed that intermittency is at work and developed a bi-fractal model to describe Alfvénic turbulence. They found that intermittency may change the spectral index of the second order structure function and this modifies the calculation of the spectral index (Carbone, 1994a). Ruzmaikin et al. (1995) found that polar Alfvénic turbulence should be described by a Kraichnan phenomenology (Kraichnan, 1965). However, the same data can be fitted also with a fluid-like scaling law (Tu et al., 1996) and, due to the relatively small amount of data, it is difficult to decide, on the basis of the second order structure function, which scaling relation describes appropriately intermittency in the solar wind.

In a further paper Carbone et al. (1995b) provided evidence for differences in the ESS scaling laws between ordinary fluid flows and solar wind turbulence. Through the analysis of different datasets collected in the solar wind and in ordinary fluid flows, it was shown that normalized scaling exponents $\zeta_{p} / \zeta_{3}$ are the same as far as $p \leq 8$ are considered. This indicates a kind of universality in the scaling exponents for the velocity structure functions. Differences between scaling exponents calculated in ordinary fluid flows and solar wind turbulence are confined to highorder moments. Nevertheless, the differences found in the datasets were related to different kind of singular structures in the model described by Equation (41). Solar wind data can be fitted by that model as soon as the most intermittent structures are assumed to be planar sheets $\mathrm{C}=1$ and $m=4$, that is a Kraichnan scaling is used. On the contrary, ordinary fluid flows can be fitted only when $\mathrm{C}=2$ and $m=3$, that is, structures are filaments and the Kolmogorov scaling have been used. However it is worthwhile to remark that differences have been found for high-order structure functions, just where measurements are unreliable.

\subsection{Probability distribution functions}

As said in Section 7.2 the statistics of turbulent flows can be characterized by the PDF of field differences over varying scales. At large scales PDFs are Gaussian, while tails become higher than Gaussian (actually, PDFs decay as $\exp \left[-\delta Z_{\ell}^{ \pm}\right]$) at smaller scales.

Marsch and Tu (1994) started to investigate the behavior of PDFs of fluctuations against scales and they found that PDFs are rather spiky at small scales and quite Gaussian at large scales. The same behavior have been obtained by Sorriso-Valvo et al. $(1999,2001)$ who investigated Helios 2 data for both velocity and magnetic field.

In order to make a quantitative analysis of the energy cascade leading to the scaling dependence of PDFs just described, the distributions obtained in the solar wind have been fitted (Sorriso-Valvo et al., 1999) by using the log-normal ansatz

$$
G_{\lambda}(\sigma)=\frac{1}{\sqrt{2 \pi} \lambda} \exp \left(-\frac{\ln ^{2} \sigma / \sigma_{0}}{2 \lambda^{2}}\right) .
$$

The width of the log-normal distribution of $\sigma$ is given by $\lambda^{2}(\ell)=\sqrt{\left\langle(\delta \sigma)^{2}\right\rangle}$, while $\sigma_{0}$ is the most probable value of $\sigma$. 
Table 5: The values of the parameters $\sigma_{0}, \mu$, and $\gamma$, in the fit of $\lambda^{2}(\tau)$ (see Equation (45) as a kernel for the scaling behavior of PDFs. FW and SW refer to fast and slow wind, respectively, as obtained from the Helios 2 spacecraft, by collecting in a single dataset all periods.

\begin{tabular}{lllll}
\hline parameter & B field $(\mathrm{SW})$ & V fiele $(\mathrm{SW})$ & B field $(\mathrm{FW})$ & V field $(\mathrm{FW})$ \\
\hline$\sigma_{0}$ & $0.90 \pm 0.05$ & $0.95 \pm 0.05$ & $0.85 \pm 0.05$ & $0.90 \pm 0.05$ \\
$\mu$ & $0.75 \pm 0.03$ & $0.38 \pm 0.02$ & $0.90 \pm 0.03$ & $0.54 \pm 0.03$ \\
$\gamma$ & $0.18 \pm 0.03$ & $0.20 \pm 0.04$ & $0.19 \pm 0.02$ & $0.44 \pm 0.05$ \\
\hline
\end{tabular}

The Equation (42) has been fitted to the experimental PDFs of both velocity and magnetic intensity, and the corresponding values of the parameter $\lambda$ have been recovered. In Figures 84 and 85 the solid lines show the curves relative to the fit. It can be seen that the scaling behavior of PDFs, in all cases, is very well described by Equation (42). At every scale $r$, we get a single value for the width $\lambda^{2}(r)$, which can be approximated by a power law $\lambda^{2}(r)=\mu r^{-\gamma}$ for $r<1 \mathrm{~h}$, as it can be seen in Figure 86. The values of parameters $\mu$ and $\gamma$ obtained in the fit, along with the values of $\sigma_{0}$, are reported in Table 5 . The fits have been obtained in the range of scales $\tau \leq 0.72 \mathrm{~h}$ for the magnetic field, and $\tau \leq 1.44 \mathrm{~h}$ for the velocity field. The analysis of PDFs shows once more that magnetic field is more intermittent than the velocity field.

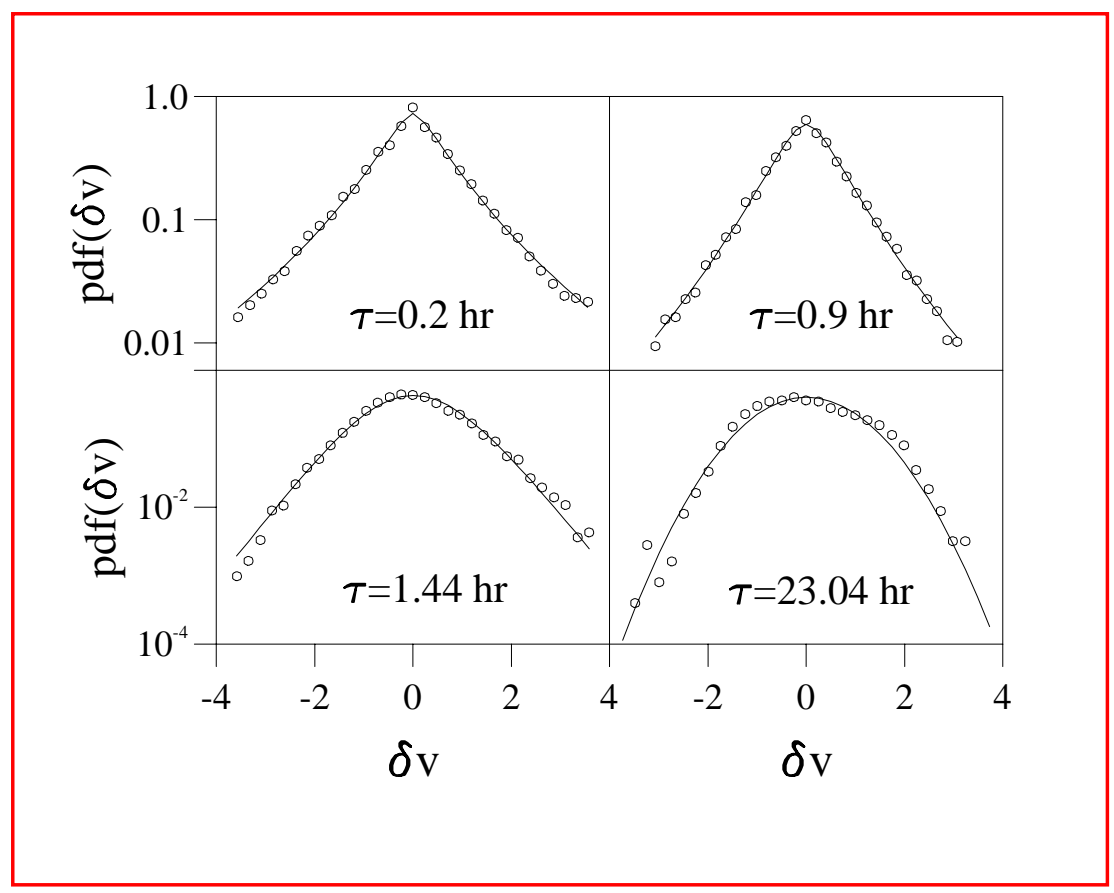

Figure 84: Normalized PDFs of fluctuations of the longitudinal velocity field at four different scales $\tau$. Solid lines represent the fit made by using the log-normal model (adopted from SorrisoValvo et al., 1999, (c) 1999 American Geophysical Union, reproduced by permission of American Geophysical Union).

The same analysis has been repeated by Forman and Burlaga (2003). These authors used $64 \mathrm{~s}$

Living Reviews in Solar Physics

http: //www. livingreviews . org/lrsp-2005-4 


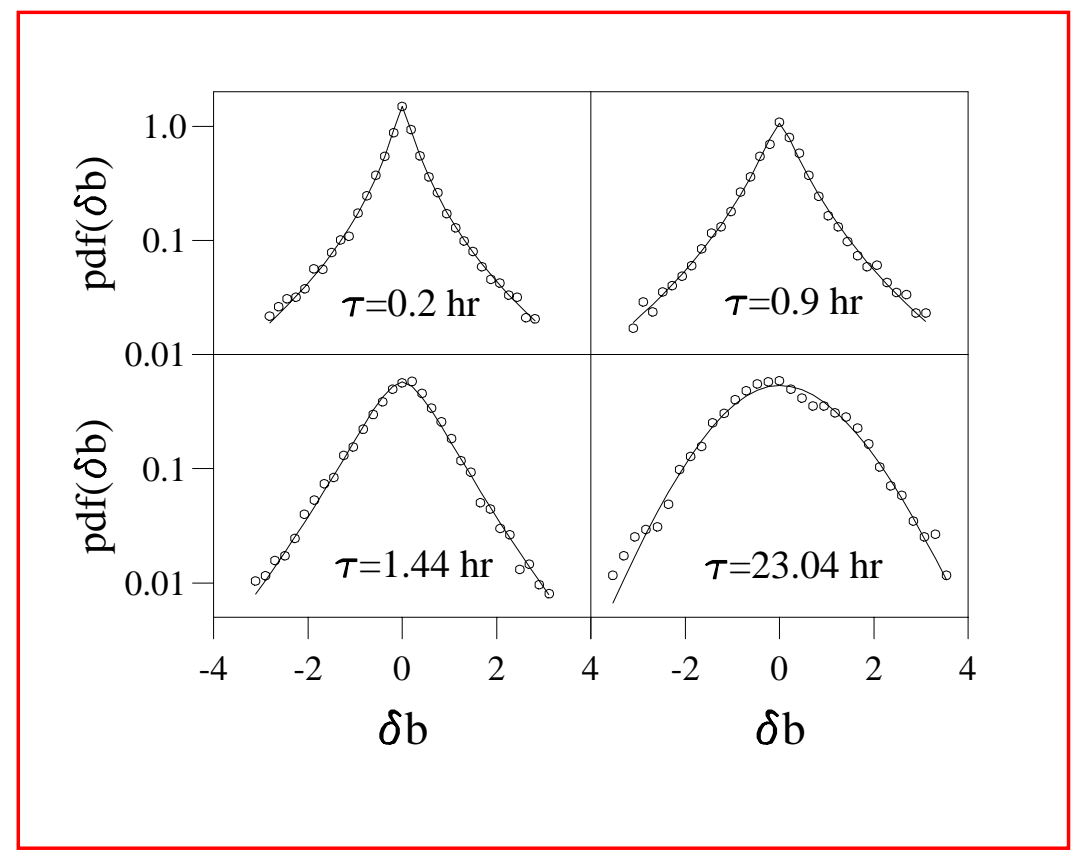

Figure 85: We show the normalized PDFs of fluctuations of the magnetic field magnitude at four different scales $\tau$ as indicated in the different panels. Solid lines represent the fit made by using the log-normal model (adopted from Sorriso-Valvo et al., 1999, (c) 1999 American Geophysical Union, reproduced by permission of American Geophysical Union).

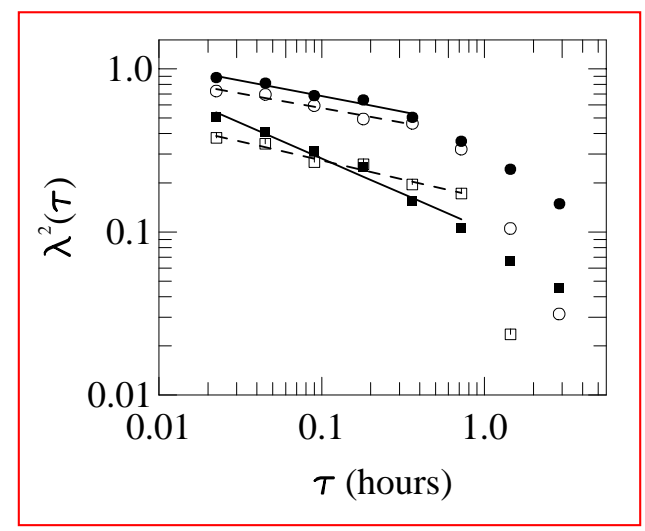

Figure 86: Scaling laws of the parameter $\lambda^{2}(\tau)$ as a function of the scales $\tau$, obtained by the fits of the PDFs of both velocity and magnetic variables (see Figures 84 and 85). Solid lines represent fits made by power laws (adopted from Sorriso-Valvo et al., 1999, (c) 1999 American Geophysical Union, reproduced by permission of American Geophysical Union). 
averages of radial solar wind speed reported by the SWEPAM instrument on the ACE spacecraft, increments have been calculated over a range of lag times from $64 \mathrm{~s}$ to several days. From the PDF obtained through the Equation (45) authors calculated the structure functions and compared the free parameters of the model with the scaling exponents of the structure functions. Then a fit on the scaling exponents allows to calculate the values of $\lambda^{2}$ and $\sigma_{0}$. Once these parameters have been calculated, the whole PDF is evaluated. The same authors found that the PDFs do not precisely fit the data, at least for large values of the moment order. Interesting enough, Forman and Burlaga (2003) investigated the behavior of PDFs when different kernels $G_{\lambda}(\sigma)$, derived from different cascade models, are taken into account in Equation (42). They discussed the physical content of each model, concluding that a cascade model derived from lognormal or log-Lévy theories ${ }^{10}$, modified by self-organized criticality proposed by Schertzer et al. (1997), seems to avoid all problems present in other cascade models.

\footnotetext{
${ }^{10}$ The log-Lévy model is a modification of the lognormal model. In such case, the central limit theorem is used to derive the limit distribution of an infinite sum of stochastic variables by relaxing the hypothesis of finite variance usually used. The resulting limit function is a Lévy function.
}

Living Reviews in Solar Physics

http://www . livingreviews . org/lrsp-2005-4 


\section{Turbulent Structures}

The non-linear energy cascade towards smaller scales accumulates fluctuations only in relatively small regions of space, where gradients become singular. As a rather different point of view (see Farge, 1992) these regions can be viewed as localized zones of fluid where phase correlation exists, in some sense coherent structures. These structures, which dominate the statistics of small scales, occur as isolated events with a typical lifetime greater than that of stochastic fluctuations surrounding them. The idea of a turbulence in the solar wind made by a mixture of structures convected by the wind and stochastic fluctuations is not particularly new (see, e.g., Tu and Marsch, 1995a). However, these large-scale structures cannot be considered as intermittent structures at all scales. Structures continuously appear and disappear apparently in a random fashion, at some random location of fluid, and carry a great quantity of energy of the flow. In this framework intermittency can be considered as the result of the occurrence of coherent (non-Gaussian) structures at all scales, within the sea of stochastic Gaussian fluctuations.

This point of view is the result of data analysis of scaling laws of turbulent fluctuations made by using wavelets filters (see Appendix 14) instead of the usual Fourier transform. Unlike the Fourier basis, wavelets allow a decomposition both in time and frequency (or space and scale). In analyzing intermittent structures it is useful to introduce a measure of local intermittency, as for example the Local Intermittency Measure (LIM) introduced by Farge (1992) (see Appendix 14).

Looking at the field $f_{s}(x)$ one can investigate the spatial behavior of structures generating intermittency. Relatively to the solar wind, the Haar basis has been applied to time series of thirteen months of velocity and magnetic data from ISEE space experiment for the first time by Veltri and Mangeney (1999a). Analyzing intermittent events, they found that intermittent events occur on time scale of the order of few minutes and that they are one-dimensional structures (in agreement with Carbone et al., 1995b). In particular, they found different types of structures which can represent two different categories:

i. Some of the structures are the well known one-dimensional current sheets, characterized by pressure balance and almost constant density and temperature. When a minimum variance analysis is made on the magnetic field near the structure, it can be seen that the most variable component of the magnetic field changes sign. This component is perpendicular to the average magnetic field, the third component being zero. An interesting property of these structures is that the correlation between velocity and magnetic field within them is opposite with respect to the rest of fluctuations. That is, when they occur during Alfvénic periods velocity and magnetic field correlation is low; on the contrary, during non-Alfvénic periods the correlation of structure increases.

ii. A different kind of structures looks like a shock wave. They can be parallel shocks or slowmode shocks. In the first case they are observed on the radial component of the velocity field, but are also seen on the magnetic field intensity, proton temperature, and density. In the second case they are characterized by a very low value of the plasma $\beta$ parameter, constant pressure, anti-correlation between density and proton temperature, no magnetic fluctuations, and velocity fluctuations directed along the average magnetic field.

Given a turbulent time series, as derived in the solar wind, a very interesting statistics can be made on the time separation between the occurrence of two consecutive structures. Let us consider a signal, for example $u(t)$ or $b(t)$ derived from solar wind, and let us define the wavelets set $w_{s}(r, t)$ as the set which captures, at time $t$, the occurrence of structures at the scale $r$. Then define the waiting times $\delta t$, as that time between two consecutive structures at the scale $r$, that is, between $w_{s}(r, t)$ and $w_{s}(r, t+\delta t)$. The PDFs of waiting times $P(\delta t)$ are reported in Figure 74 . As it can be seen, waiting times are distributed according to a power law $P(\delta t) \sim \delta t^{-\beta}$ extended over at least two decades. This property is very interesting, because this means that the underlying process for 
the energy cascade is non-Poissonian. Waiting times occurring between isolated Poissonian events, must be distributed according to an exponential function. The power law for $P(\delta t)$ represents the asymptotic behavior of a Lévy function with characteristic exponent $\alpha=\beta-1$. This describes self-affine processes and are obtained from the central limit theorem by relaxing the hypothesis that the variance of variables is finite. The power law for waiting times we found is a clear evidence that long-range correlation (or in some sense "memory") exists in the underlying cascade process.

On the other hand, Bruno et al. (2001), analyzing the statistics of the occurrence of waiting times of magnetic field intensity and wind speed intermittent events for a short time interval within the trailing edge of a high velocity stream, found a possible Poissonian-like behavior with a characteristic time around $30 \mathrm{~min}$ for both magnetic field and wind speed. These results recalled previous estimates of the occurrence of interplanetary discontinuities performed by Tsurutani and Smith (1979), who found a waiting time around 14 min. In addition, Bruno et al. (2001), taking into account the wind speed and the orientation of the magnetic field vector at the site of the observation, in the hypothesis of spherical expansion, estimated the corresponding size at the Sun surface that resulted to be of the order of the photospheric structures estimated also by Thieme et al. (1989). Obviously, the Poissonian statistics found by these authors does not agree with the clear power law shown in Figure 74. However, Bruno et al. (2001) included intermittent events found at all scales while results shown in Figure 74 refer to waiting times between intermittent events extracted at the smallest scale, which results to be about an order of magnitude smaller than the time resolution used by Bruno et al. (2001). A detailed study on this topic would certainly clarify possible influences on the waiting time statistics due to the selection of intermittent events according to the corresponding scale.

In the same study by Bruno et al. (2001), these authors analyzed in detail an event characterized by a strong intermittent signature in the magnetic field intensity. A comparative study was performed choosing a close-by time interval that, although intermittent in velocity, was not characterized by strong magnetic intermittency. This time interval was located a few hours apart from the previous one. These two intervals are indicated in Figure 87 by the two vertical boxes labeled 1 and 2, respectively. Wind speed profile and magnetic field magnitude are shown in the first two panels. In the third panel, the blue line refers to the logarithmic value of the magnetic pressure $P_{m}$, here indicated by $P_{B}$; the red line refers to the logarithmic value of the thermal pressure $P_{k}$, here indicated by $P_{K}$ and the black line refers to the logarithmic value of the total pressure $P_{\text {tot }}$, here indicated by $P_{\mathrm{T}}=P_{B}+P_{K}$, including an average estimate of the electrons and $\alpha$ s contributions. Magnetic field intensity residuals, obtained from the LIM technique, are shown in the bottom panel. The first interval is characterized by strong magnetic field intermittency while the second one is not. In particular, the first event corresponds to a relatively strong field discontinuity which separates two regions characterized by a different bulk velocity and different level of total pressure. While kinetic pressure (red trace) does not show any major jump across the discontinuity but only a light trend, magnetic pressure (blue trace) clearly shows two distinct levels.

A minimum variance analysis further reveals the intrinsic different nature of these two intervals as shown in Figure 88 where original data have been rotated into the field minimum variance reference system (see Appendix 15.1) where maximum, intermediate and minimum variance components are identified by $\lambda_{3}, \lambda_{2}$, and $\lambda_{1}$, respectively. Moreover, at the bottom of the column we show the hodogram on the maximum variance plane $\lambda_{3}-\lambda_{2}$, as a function of time on the vertical axis.

The good correlation existing between magnetic and velocity variations for both time intervals highlights the presence of Alfvénic fluctuations. However, only within the first interval the magnetic field vector describes an arc-like structure larger than $90^{\circ}$ on the maximum variance plane (see rotation from A to B on the 3D graph at the bottom of the left column in Figure 88) in correspondence with the time interval identified, in the profile of the magnetic field components, by the

Living Reviews in Solar Physics

http://www . livingreviews . org/Irsp-2005-4 


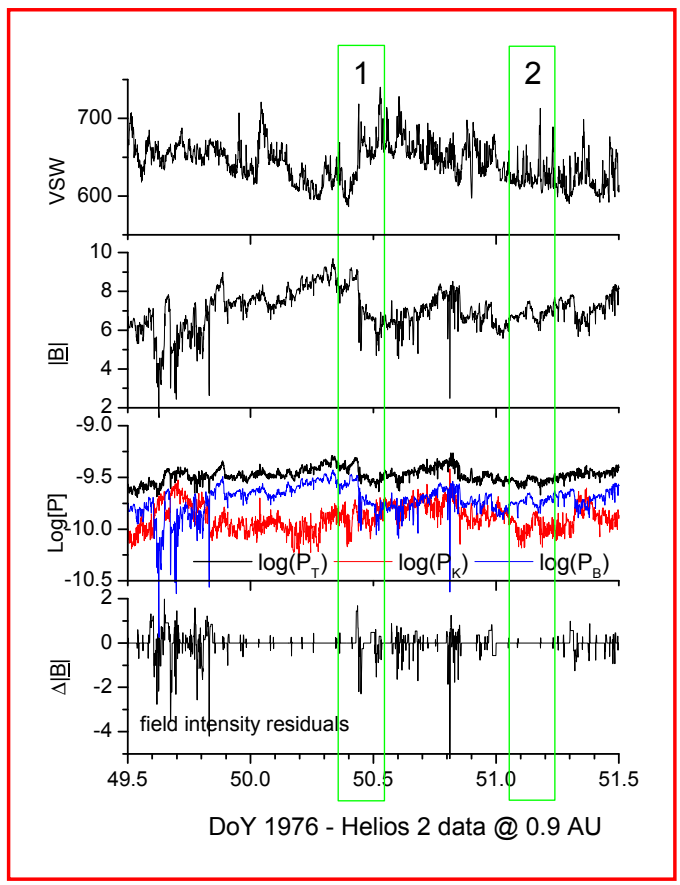

Figure 87: From top to bottom, we show $81 \mathrm{~s}$ averages of velocity wind profile in $\mathrm{km} \mathrm{s}^{-1}$, magnetic field intensity in $\mathrm{nT}$, the logarithmic value of magnetic (blue line), thermal(red line), and total pressure (black line) in dyne $/ \mathrm{cm}^{2}$ and field intensity residuals in $\mathrm{nT}$. The two vertical boxes delimit the two time intervals \# 1 and \#2 which were chosen for comparison. While the first interval shows strong magnetic intermittency, the second one does not (adopted from Bruno et al., 2001). 
green color. At this location, the magnetic field intensity shows a clear discontinuity, $B\left[\lambda_{3}\right]$ changes sign, $B\left[\lambda_{2}\right]$ shows a hump whose maximum is located where the previous component changes sign and, finally, $B\left[\lambda_{1}\right]$ keeps its value close to zero across the discontinuity. Velocity fluctuations are well correlated with magnetic field fluctuations and, in particular, the minimum variance component $V\left[\lambda_{1}\right]$ has the same value on both sides of the discontinuity, approximately $350 \mathrm{~km} \mathrm{~s}^{-1}$, indicating that there is no mass flux through the discontinuity. During this interval, which lasts about $26 \mathrm{~min}$, the minimum variance direction lies close to the background magnetic field direction at $11.9^{\circ}$ so that the arc is essentially described on a plane perpendicular to the average background magnetic field vector. However, additional although smaller and less regular arc-like structures can be recognized on the maximum variance plane $\lambda_{2}-\lambda_{3}$, and they tend to cover the whole $2 \pi$.

Within the second interval, magnetic field intensity is rather constant and the three components do not show any particular fluctuation, which could resemble any sort of rotation. In other words, the projection on the maximum variance plane does not show any coherent path. Even in this case, these fluctuations happen to be in a plane almost perpendicular to the average field direction since the angle between this direction and the minimum variance direction is about $9.3^{\circ}$.

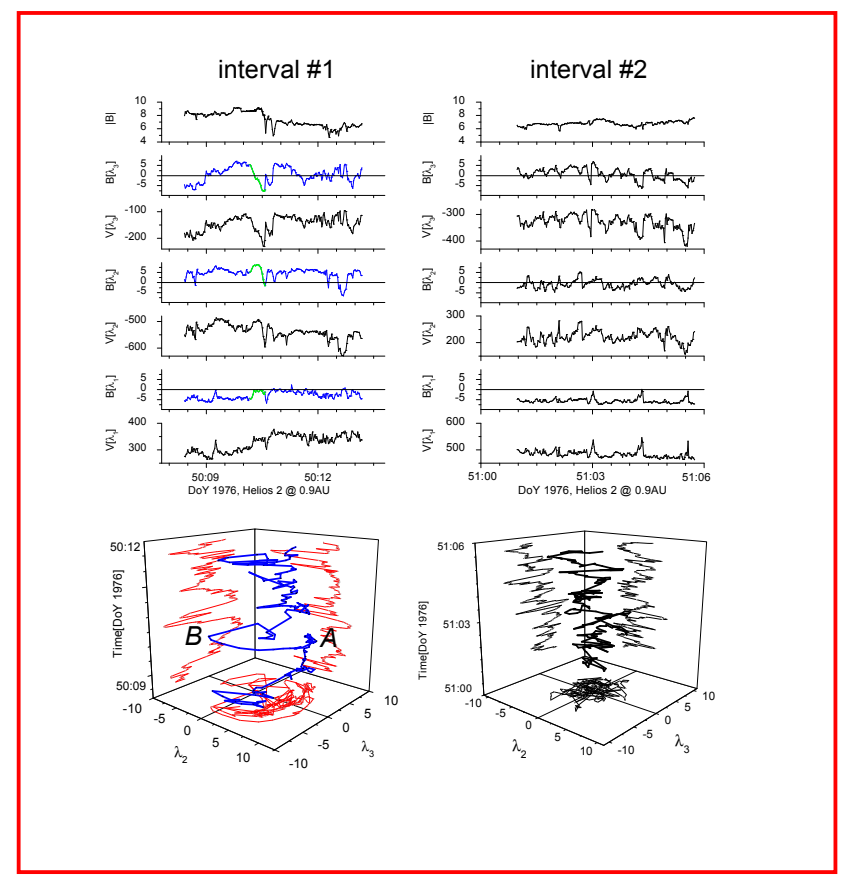

Figure 88: Left column, from top to bottom: we show magnetic field intensity, maximum $\lambda_{3}$, intermediate $\lambda_{2}$ and minimum $\lambda_{1}$ variance components for magnetic field (blue color) and wind velocity relative to the time interval \#1 shown in Figure 87. Right below, we show the hodogram on the maximum variance plane $\lambda_{3}-\lambda_{2}$, as a function of time (blue color line). The red lines are the projection of the blue line. The large arc, from $A$ to $B$, corresponds to the green segment in the profile of the magnetic field components shown in the upper panel. The same parameters are shown for interval \# 2 (Figure 87), in the same format, on the right hand side of the figure. The time resolution of the data is $81 \mathrm{~s}$ (adopted from Bruno et al., 2001).

Further insights about differences between these two intervals can be obtained when we plot the trajectory followed by the tip of the magnetic field vector in the minimum variance reference

Living Reviews in Solar Physics

http: //www. livingreviews . org/lrsp-2005-4 
system, as shown in Figure 89. The main difference between these two plots is that the one relative to the first interval shows a rather patchy trajectory with respect to the second interval. As a matter of fact, if we follow the displacements of the tip of the vector as the time goes by, we observe that the two intervals have a completely different behavior.

Within the first time interval, the magnetic field vector experiences for some time small displacements around a given direction in space and then it suddenly performs a much larger displacement towards another direction in space, about which it starts to wander again. This process keeps on going several times within this time interval. In particular, the thick green line extending from label A to label B refers to the arc-like discontinuity shown in Figure 88, which is also the largest directional variation within this time interval. Within the second interval, the vector randomly fluctuates in all direction and, as a consequence, both the 3D trajectory and its projection on the maximum variance plane do not show any large empty spot. In practice, the second time interval, although longer, is similar to any sub-interval corresponding to one of the trajectory patches recognizable in the left hand side panel. As a matter of fact, selecting a single patch from the first interval and performing a minimum variance analysis, the maximum variance plane would result to be perpendicular to the local average magnetic field direction and the tip of the vector would randomly fluctuate in all directions. The first interval can be seen as a collection of several sub-intervals similar to interval \# 2 characterized by different field orientations and, possibly, intensities. Thus, magnetic field intermittent events mark the border between adjacent intervals populated by stochastic Alfvénic fluctuations.

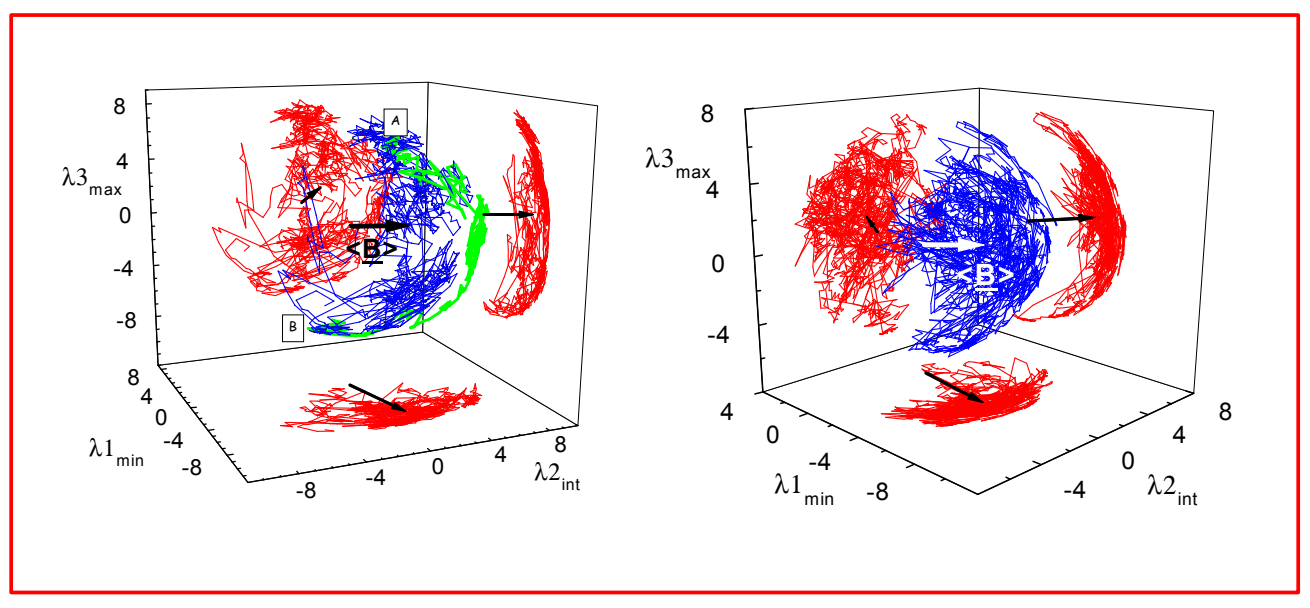

Figure 89: Trajectory followed by the tip of the magnetic field vector (blue color line) in the minimum variance reference system for interval \# 1 (left) and \# 2 (right). Projections on the three planes (red color lines) formed by the three eigenvectors $\lambda_{1}, \lambda_{2}, \lambda_{3}$, and the average magnetic field vector, with its projections on the same planes, are also shown. The green line extending from label A to label B refers to the arc-like discontinuity shown in Figure 88. The time resolution of the magnetic field averages is $6 \mathrm{~s}$ (adopted from Bruno et al., 2001). (To see animations relative to similar time intervals click on Figures 90 for a time series affected by the intermittency phenomenon or at 91 for non-intermittent and intermittent samples.

These differences in the dynamics of the orientation of the field vector can be appreciated running the two animations behind Figures 90 and 91. Although the data used for these movies do not exactly correspond to the same time intervals analyzed in Figure 87, they show the same dynamics that the field vector has within intervals \# 1 and \# 2. In particular, the animation 


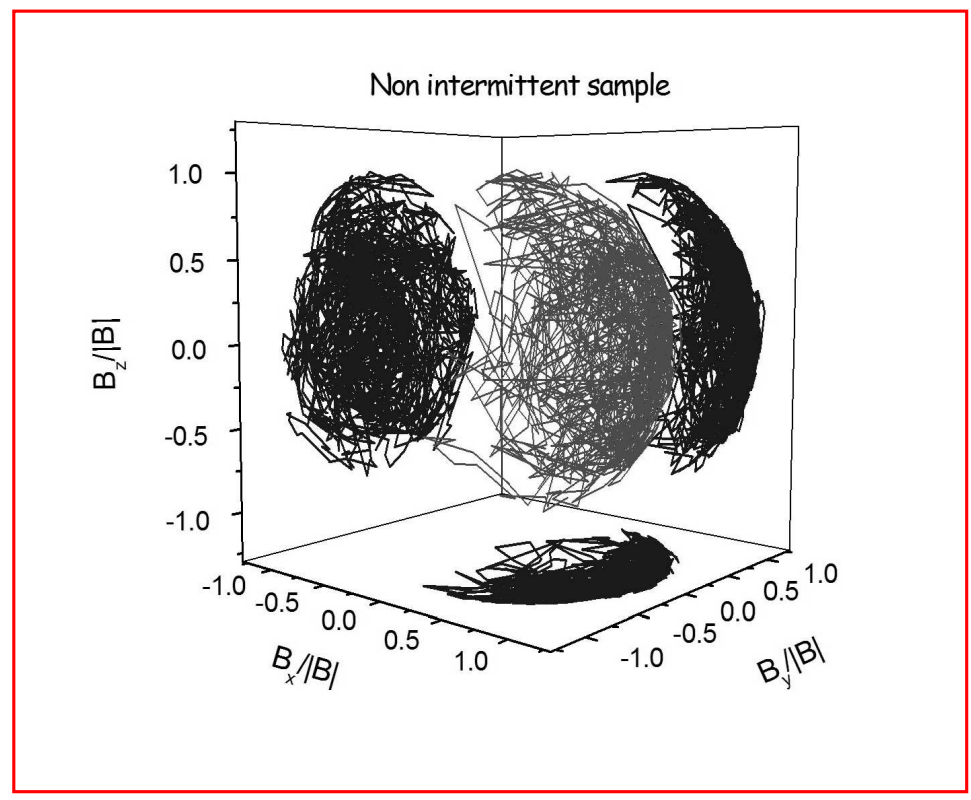

Figure 90: Still from a Trajectory followed by the tip of the magnetic field vector in the minimum variance reference system during a time interval not characterized by intermittency. The duration of the interval is $2000 \times 6 \mathrm{~s}$ but the magnetic field vector moves only for $100 \times 6 \mathrm{~s}$ in order to make a smaller file (movie kindly provided by A. Vecchio). (To watch the movie, please go to the online version of this review article at http://www.livingreviews.org/lrsp-2005-4.)

Living Reviews in Solar Physics

http://www . livingreviews .org/lrsp-2005-4 
corresponding to Figure 90 represents interval \# 2 while, Figure 91 represents interval \# 1.

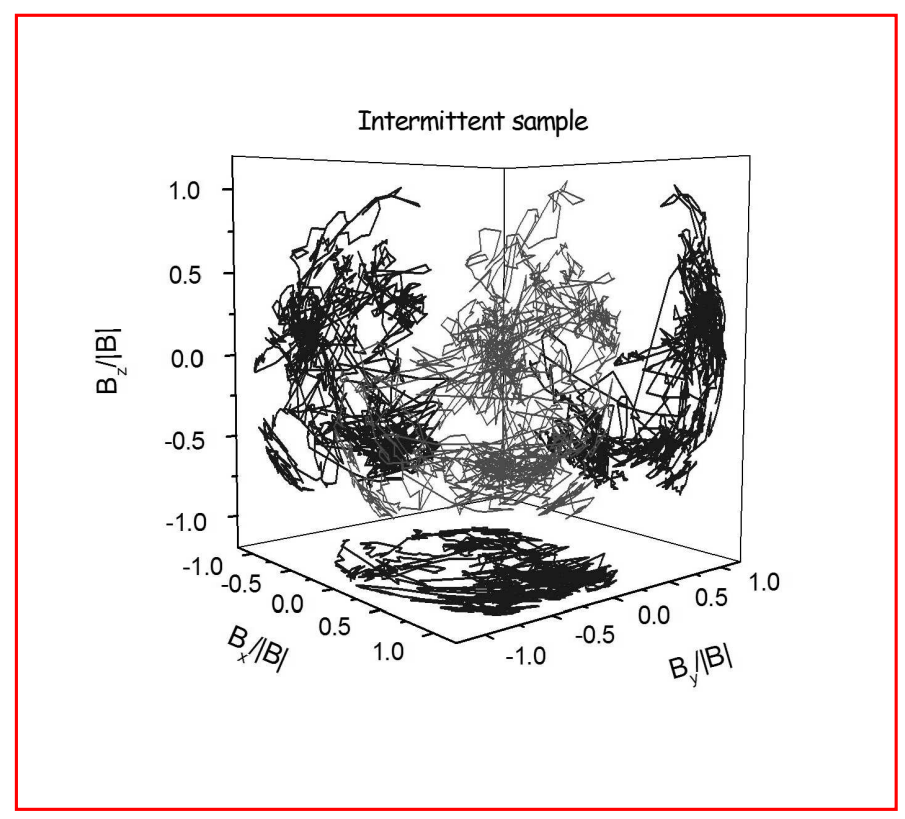

Figure 91: Still from a Trajectory followed by the tip of the magnetic field vector in the minimum variance reference system during a time interval characterized by intermittent events. The duration of the interval is $2000 \times 6 \mathrm{~s}$ but the magnetic field vector moves only for $100 \times 6 \mathrm{~s}$ in order to make a smaller file (movie kindly provided by A. Vecchio). (To watch the movie, please go to the online version of this review article at http://www.livingreviews.org/lrsp-2005-4.)

The observations reported above suggested these authors to draw the sketch shown in Figure 92 that shows a simple visualization of hypothetical flux tubes, convected by the wind, which tangle up in space. Each flux tube is characterized by a local field direction and intensity, and within each flux tube the presence of Alfvénic fluctuations makes the magnetic field vector randomly wander about this direction. Moreover, the large scale is characterized by an average background field direction aligned with the local interplanetary magnetic field. This view, based on the idea that solar wind fluctuations are a superposition of propagating Alfvén waves and convected structures (Bavassano and Bruno, 1989), strongly recalls the work by Tu and Marsch (1990a, 1993) who suggested the solar wind fluctuations being a superposition of pressure balance structure (PBS) type flux tubes and Alfvén waves. In the inner heliosphere these PBS-type flux tubes are embedded in the large structure of fast solar wind streams and would form a kind of spaghetti-like sub-structure, which probably has its origin at the base of the solar atmosphere.

The border between these flux tubes can be a tangential discontinuity where the total pressure on both sides of the discontinuity is in equilibrium or, as in the case of interval \#1, the discontinuity is located between two regions not in pressure equilibrium. If the observer moves across these tubes he will record the patchy configuration shown in Figure 91 relative to interval \#1. Within each flux tube he will observe a local average field direction and the magnetic field vector would mainly fluctuate on a plane perpendicular to this direction. Moving to the next tube, the average field direction would rapidly change and magnetic vector fluctuations would cluster around this new direction. Moreover, if we imagine a situation with many flux-tubes, each one characterized by a different magnetic field intensity, moving across them would possibly increase the intermittent level 
of the fluctuations. On the contrary, moving along a single flux tube, the same observer would constantly be in the situation typical of interval \#2, which is mostly characterized by a rather constant magnetic field intensity and directional stochastic fluctuations mainly on a plane quasi perpendicular to the average magnetic field direction. In such a situation, magnetic field intensity fluctuations would not increase their intermittency.

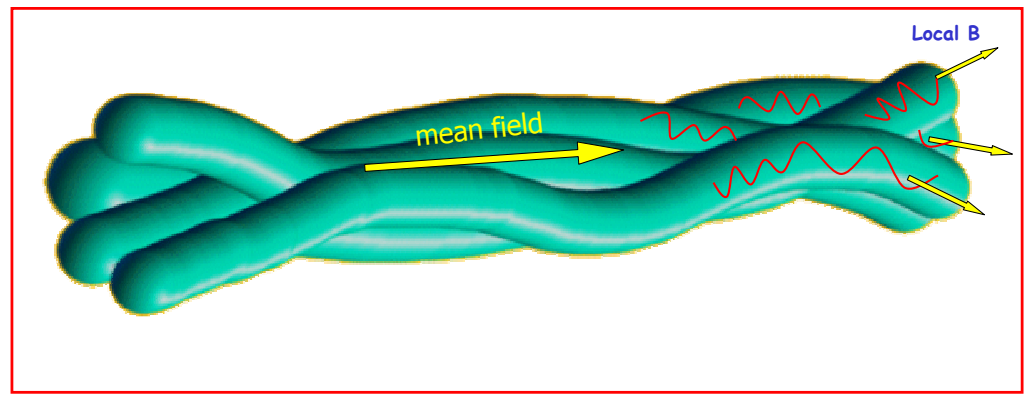

Figure 92: Simple visualization of hypothetical flux tubes which tangle up in space. Each flux tube is characterized by a local field direction, and within each flux tube the presence of Alfvénic fluctuations makes the magnetic field vector randomly wander about this direction. Moreover, the large scale is characterized by an average background field direction aligned with the local interplanetary magnetic field. Moving across different flux-tubes, characterized by a different values of $|B|$, enhances the intermittency level of the magnetic field intensity time series (adopted from Bruno et al., 2001).

A recent theoretical effort by Chang et al. (2004), Chang (2003), and Chang and Wu (2002) models MHD turbulence in a way that recalls the interpretation of the interplanetary observations given by Bruno et al. (2001) and, at the same time, reminds also the point of view expressed by Farge (1992) in this section. These authors stress the fact that propagating modes and coherent, convected structures share a common origin within the general view described by the physics of complexity. Propagating modes experience resonances which generate coherent structures, possibly flux tubes, which, in turn, will migrate, interact, and, eventually, generate new modes. This process, schematically represented in Figure 93, which favors the local generation of coherent structures in the solar wind, fully complement the possible solar origin of the convected component of interplanetary MHD turbulence.

\subsection{Radial evolution of intermittency in the ecliptic}

Marsch and Liu (1993) investigated for the first time solar wind scaling properties in the inner heliosphere. These authors provided some insights on the different intermittent character of slow and fast wind, on the radial evolution of intermittency, and on the different scaling characterizing the three components of velocity. In particular, they found that fast streams were less intermittent than slow streams and the observed intermittency showed a weak tendency to increase with heliocentric distance. They also concluded that the Alfvénic turbulence observed in fast streams starts from the Sun as self-similar but then, during the expansion, decorrelates becoming more multifractal. This evolution was not seen in the slow wind, supporting the idea that turbulence in fast wind is mainly made of Alfvén waves and convected structures (Tu and Marsch, 1993), as already inferred by looking at the radial evolution of the level of cross-helicity in the solar wind (Bruno and Bavassano, 1991).

Bruno et al. (2003a) investigated the radial evolution of intermittency in the inner heliosphere, using the behavior of the flatness of the PDF of magnetic field and velocity fluctuations as a function

Living Reviews in Solar Physics

http: //www. livingreviews . org/lrsp-2005-4 


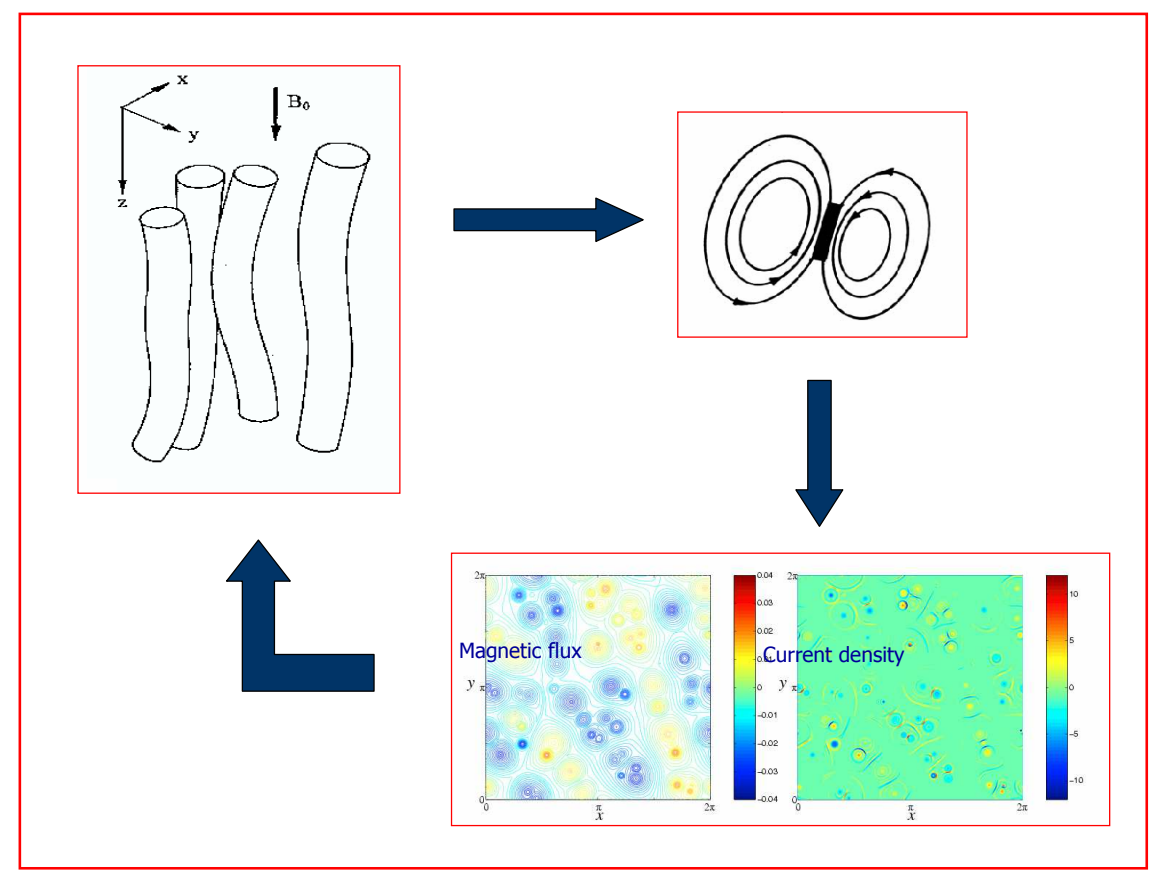

Figure 93: Composite figure made adapting original figures from the paper by Chang et al. (2004). The first element on the upper left corner represents field-aligned spatio-temporal coherent structures. A cross-section of two of these structures of the same polarity is shown in the upper right corner. Magnetic flux iso-contours and field polarity are also shown. The darkened area represents intense current sheet during strong magnetic shear. The bottom element of the figure is the result of 2D MHD simulations of interacting coherent structures, and shows intermittent spatial distribution of intense current sheets. In this scenario, new fluctuations are produced which can provide new resonance sites, possibly nucleating new coherent structures 
of scale. As a matter of fact, probability distribution functions of fluctuating fields affected by intermittency become more and more peaked at smaller and smaller scales. Since the peakedness of a distribution is measured by its flatness factor, they studied the behavior of this parameter at different scales to estimate the degree of intermittency of their time series, as suggested by Frisch (1995).

In order to study intermittency they computed the following estimator of the flatness factor $\mathcal{F}$ :

$$
\mathcal{F}(\tau)=\frac{<S_{\tau}^{4}>}{<S_{\tau}^{2}>^{2}}
$$

where $\tau$ is the scale of interest and $S_{\tau}^{p}=<|V(t+\tau)-V(t)|^{p}>$ is the structure function of order $p$ of the generic function $V(t)$. They considered a given function to be intermittent if the factor $\mathcal{F}$ increased when considering smaller and smaller scales or, equivalently, higher and higher frequencies.

In particular, vector field, like velocity and magnetic field, encompasses two distinct contributions, a compressive one due to intensity fluctuations that can be expressed as $\delta|\mathbf{B}(t, \tau)|=$ $|\mathbf{B}(t+\tau)|-|\mathbf{B}(t)|$, and a directional one due to changes in the vector orientation $\delta \mathbf{B}(t, \tau)=$ $\sqrt{\sum_{i=x, y, z}\left(B_{i}(t+\tau)-B_{i}(t)\right)^{2}}$. Obviously, relation $\delta \mathbf{B}(t, \tau)$ takes into account also compressive contributions, and the expression $\delta \mathbf{B}(t, \tau) \geq|\delta| \mathbf{B}(t, \tau)||$ is always true.

Looking at Figures 94 and 95, taken from the work of Bruno et al. (2003a), the following conclusions can be drawn:

- Magnetic field fluctuations are more intermittent than velocity fluctuations.

- Compressive fluctuations are more intermittent than directional fluctuations.

- Slow wind intermittency does not show appreciable radial dependence.

- Fast wind intermittency, for both magnetic field and velocity, clearly increases with distance.

- Magnetic and velocity fluctuations have a rather Gaussian behavior at large scales, as expected, regardless of type of wind or heliocentric distance.

Moreover, they also found that the intermittency of the components rotated into the mean field reference system (see Appendix 15.1) showed that the most intermittent component of the magnetic field is the one along the mean field, while the other two show a similar level of intermittency within the associated uncertainties. This different behavior is then enhanced for larger heliocentric distances. These results agree with conclusions drawn by Marsch and Tu (1994) who, analyzing fast and slow wind at $0.3 \mathrm{AU}$, found that the PDFs of the fluctuations of transverse components of both velocity and magnetic fields, constructed for different time scales, were appreciably more Gaussian-like than fluctuations observed for the radial component, which resulted to be more and more spiky for smaller and smaller scales. However, this difference between radial and transverse components seemed to vanish with increasing heliocentric distance, and Tu et al. (1996) could not establish a clear radial trend or anisotropy. These results might be reconciled with conclusions by Bruno et al. (2003b) if the analysis by Tu et al. (1996) was repeated in the mean field reference system. The reason is that components normal to the mean field direction are more influenced by Alfvénic fluctuations and, as a consequence, their fluctuations are more stochastic and less intermittent. This effect largely reduces during the radial excursion mainly because the Solar Ecliptic (SE) reference system is not the most appropriate one for studying magnetic field fluctuations, and a cross-talking between different components is artificially introduced. As a matter of fact, the presence of the large scale spiral magnetic field breaks the spatial symmetry introducing a preferential direction parallel to the mean field. The same Bruno et al. (2003b) showed that it

Living Reviews in Solar Physics

http: //www. livingreviews . org/lrsp-2005-4 


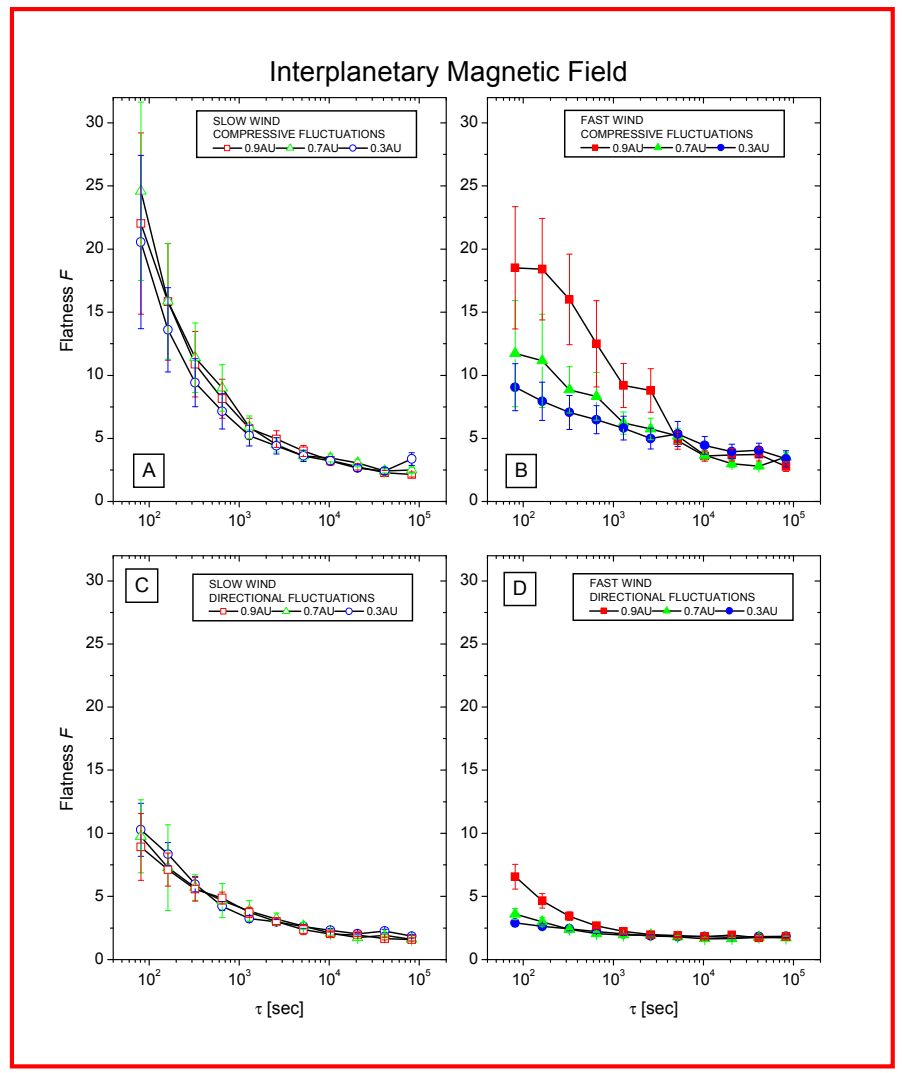

Figure 94: Flatness $\mathcal{F}$ vs. time scale $\tau$ relative to magnetic field fluctuations. The left column (panels A and C) refers to slow wind and the right column (panels B and D) refers to fast wind. The upper panels refer to compressive fluctuations and the lower panels refer to directional fluctuations. Vertical bars represent errors associated with each value of $\mathcal{F}$. The three different symbols in each panel refer to different heliocentric distances as reported in the legend (adopted from Bruno et al., 2003b, (c) 2003 American Geophysical Union, reproduced by permission of American Geophysical Union). 


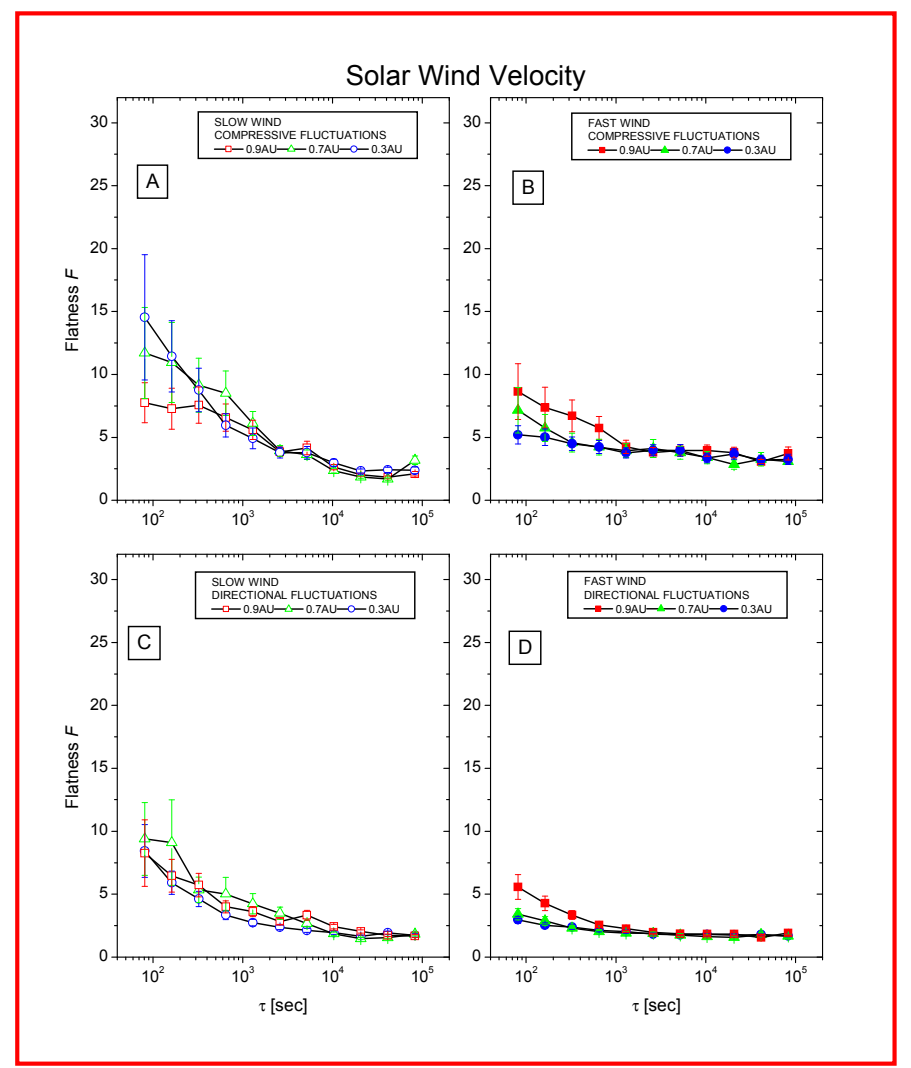

Figure 95: Flatness $\mathcal{F}$ vs. time scale $\tau$ relative to wind velocity fluctuations. In the same format of Figure 94 panels A and C refer to slow wind and panels B and D refer to fast wind. The upper panels refer to compressive fluctuations and the lower panels refer to directional fluctuations. Vertical bars represent errors associated with each value of $\mathcal{F}$ (adopted from Bruno et al., 2003b, (C) 2003 American Geophysical Union, reproduced by permission of American Geophysical Union). 
was not possible to find a clear radial trend unless magnetic field data were rotated into this more natural reference system.

On the other hand, it looks more difficult to reconcile the radial evolution of intermittency found by Bruno et al. (2003b) and Marsch and Liu (1993) in fast wind with conclusions drawn by Tu et al. (1996), who stated that "Neither a clear radial evolution nor a clear anisotropy can be established. The value of P1 in high-speed and low-speed wind are not prominent different.". However, it is very likely that the conclusions given above are related with how to deal with the flat slope of the spectrum in fast wind near 0.3 AU. Tu et al. (1996) concluded, indeed: "It should be pointed out that the extended model cannot be used to analyze the intermittency of such fluctuations which have a flat spectrum. If the index of the power spectrum is near or less than unity ... P1 would be 0.5. However, this does not mean there is no intermittency. The model simply cannot be used in this case, because the structure function(1) does not represent the effects of intermittency adequately for those fluctuations which have a flat spectrum and reveal no clear scaling behavior".

Bruno et al. (2003a) concluded that the two major ingredients of interplanetary MHD fluctuations are compressive fluctuations due to a sort of underlying, coherent structure convected by the wind, and stochastic Alfvénic fluctuations propagating in the wind. Depending on the type of solar wind sample and on the heliocentric distance, the observed scaling properties would change accordingly. In particular, the same authors suggested that, as the radial distance increases, convected, coherent structures of the wind assume a more relevant role since the Alfvénic component of the fluctuations is depleted. This would be reflected in the increased intermittent character of the fluctuations. The coherent nature of the convected structures would contribute to increase intermittency while the stochastic character of the Alfvénic fluctuations would contribute to decrease it. This interpretation would also justify why compressive fluctuations are always more intermittent than directional fluctuations. As a matter of fact, coherent structures would contribute to the intermittency of compressive fluctuations and, at the same time, would also produce intermittency in directional fluctuations. However, since directional fluctuations are greatly influenced by Alfvénic stochastic fluctuations, their intermittency will be more or less reduced depending on the amplitude of the Alfvén waves with respect to the amplitude of compressive fluctuations.

The radial dependence of the intermittency behavior of solar wind fluctuations stimulated Bruno et al. (1999b) to reconsider previous investigations on fluctuations anisotropy reported in Section 3.1.3. These authors studied magnetic field and velocity fluctuations anisotropy for the same corotating, high velocity stream observed by Bavassano et al. (1982a) within the framework of the dynamics of non-linear systems. Using the Local Intermittency Measure (Farge et al., 1990; Farge, 1992), Bruno et al. (1999b) were able to justify the controversy between results by Klein et al. (1991) in the outer heliosphere and Bavassano et al. (1982a) in the inner heliosphere. Exploiting the possibility offered by this technique to locate in space and time those events which produce intermittency, these authors were able to remove intermittent events and perform again the anisotropy analysis. They found that intermittency strongly affected the radial dependence of magnetic fluctuations while it was less effective on velocity fluctuations. In particular, after intermittency removal, the average level of anisotropy decreased for both magnetic and velocity field at all distances. Although magnetic fluctuations remained more anisotropic than their kinetic counterpart, the radial dependence was eliminated. On the other hand, the velocity field anisotropy showed that intermittency, although altering the anisotropic level of the fluctuations, does not markedly change its radial trend.

\subsection{Radial evolution of intermittency at high latitude}

Recently, Pagel and Balogh (2003) studied intermittency in the outer heliosphere using Ulysses observations at high heliographic latitude, well within high speed solar wind. In particular, these 
authors used Castaing distribution Castaing et al. (2001) to study the Probability Density Functions (PDF) of the fluctuations of magnetic field components. They found that intermittency of small scales fluctuations, within the inertial range, increased with increasing the radial distance from the Sun as a consequence of the growth to larger scales of the inertial range.

As a matter of fact, using the scaling found by Horbury et al. (1996a) between the transition scale (the inverse of the frequency corresponding to the break-point in the magnetic field spectrum) $T_{B} \sim r^{1.1 \pm 0.1}$, Pagel and Balogh (2003) quantitatively evaluated how the top of the inertial range in their data should shift to larger time scales with increasing heliocentric distance. Moreover, taking into account that inside the inertial range $\lambda^{2} \sim \tau^{-\beta} \Longrightarrow \lambda^{2}=a \tau^{-\beta}$ and that the proposed scaling from Castaing et al. (2001) would be $\lambda^{2} \sim$ const. $(\tau / T)^{-\beta}$, we should expect that for $\tau=T$ the parameter $\lambda^{2}=$ const.. Thus, these authors calculated $\sigma^{2}$ and $\lambda^{2}$ at different heliocentric distances and made the hypothesis of a similar scaling for $\sigma^{2}$ and $\lambda^{2}$, although this is not assured by the model. Figure 96 reports values of $\lambda^{2}$ and $\sigma^{2}$ vs. distance calculated for the top of the inertial range at that distance using the above procedure. The radial behavior shown in this figure suggests that there is no radial dependence for these parameters for all the three components (indicated by different symbols), as expected if the observed radial increase of intermittency in the inertial range is due to a broadening of the inertial range itself.

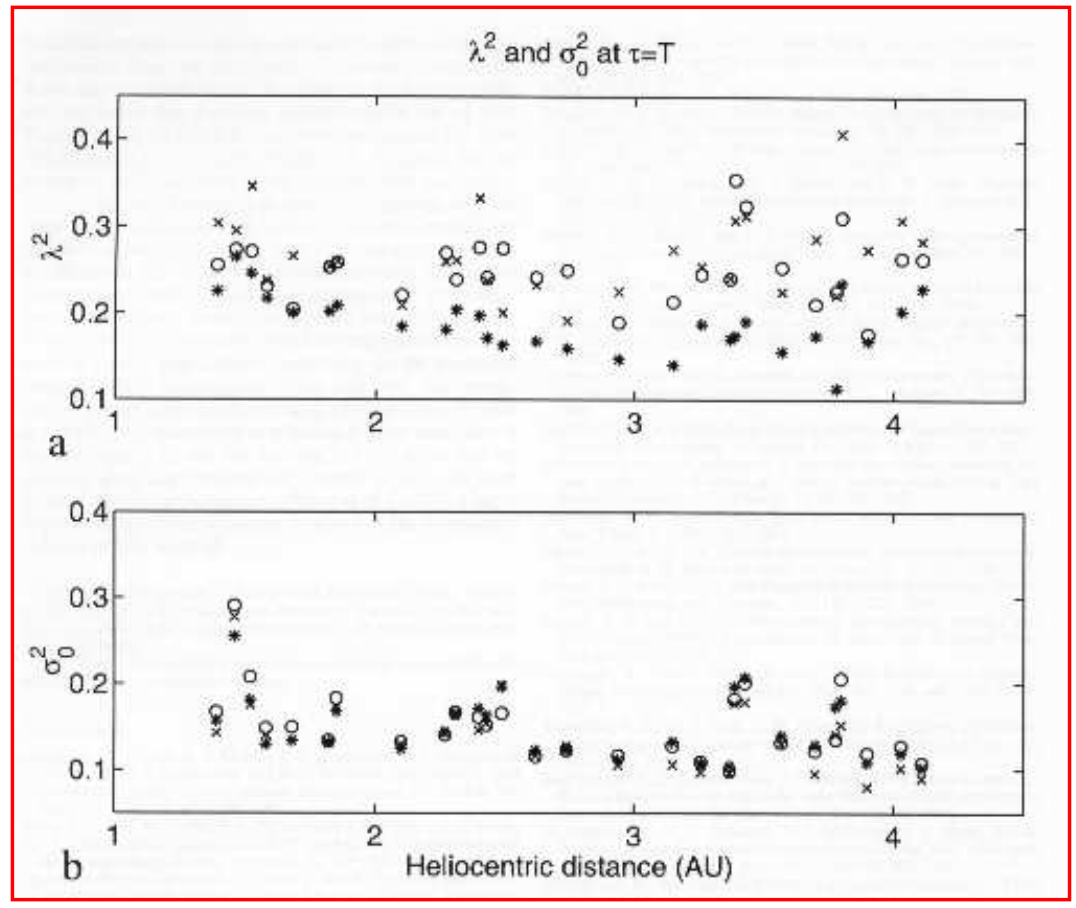

Figure 96: Values of $\lambda^{2}$ (upper panel) and $\sigma^{2}$ (lower panel) vs. heliocentric distance. These values have been calculated for the projected low frequency beginning of the inertial range relative to each distance (see text for details). R, T, and N components are indicated by asterisks, crosses and circles, respectively (adopted from Pagel and Balogh, 2003, (c) 2003 American Geophysical Union, reproduced by permission of American Geophysical Union).

They also found that, in the RTN reference system, transverse magnetic field components exhibit less Gaussian behavior with respect to the radial component. This result should be compared with results from similar studies by Marsch and Tu (1994) and Bruno et al. (2003b) who, studying

Living Reviews in Solar Physics

http: //www. livingreviews . org/lrsp-2005-4 
the radial evolution of intermittency in the ecliptic, found that the components transverse to the local magnetic field direction, are the most Gaussian ones. Probably, the above discrepancy depends totally on the reference system adopted in these different studies and it would be desirable to perform a new comparison between high and low latitude intermittency in the mean-field reference system.

Pagel and Balogh (2002) focused also on the different intermittent level of magnetic field fluctuations during two fast latitudinal scans which happened to be during solar minimum the first one, and during solar maximum the second one. Their results showed a strong latitudinal dependence but were probably not, or just slightly, affected by radial dependence given the short heliocentric radial variations during these time intervals. They analyzed the anomalous scaling of the third order magnetic field structure functions looking at the value of the parameter $\mu$ obtained from the best fit performed using the p-model (see Section 8.1). In a previous analysis of the same kind, but focused on the first latitudinal scan, the same authors tested three intermittency models, namely: "lognormal", "p" and "G-infinity" models. In particular, this last model was an empirical model introduced by Pierrehumbert (1999) and Cho et al. (2000) and was not intended for turbulent systems. Anyhow, the best fits were obtained with the lognormal and Kolmogorov-p model. These authors concluded that magnetic field components display a very high level of intermittency throughout minimum and maximum phases of solar cycle, and slow wind shows a lower level of intermittency compared with the Alfvénic polar flows. These results do not seem to agree with ecliptic observations (Marsch and Liu, 1993; Bruno et al., 2003a) which showed that fast wind is generally less intermittent than slow wind not only for wind speed and magnetic field magnitude, but also for the components. At this point, since it has been widely recognized that low latitude fast wind collected within corotating streams and fast polar wind share many common turbulence features, they should be expected to have many similarities also as regards intermittency. Thus, it is possible that also in this case the reference system in which the analysis is performed plays some role in determining some of the results regarding the behavior of the components. In any case, further analyses should clarify the reasons for this discrepancy. 


\section{Conclusions and Remarks}

Now that the reader finally reached the conclusions, hoping that he was so patient to read the whole paper, we suggest him to go back for a moment to the List of Contents, not to start all over again, but just to take a look at the various problems that have been briefly touched by this review. He will certainly realize how complex is the phenomenon of turbulence in general and, in particular, in the solar wind. Almost four decades of observations and theoretical efforts have not yet been sufficient to fully understand how this natural and fascinating phenomenon really works in the solar wind. We certainly are convinced that we cannot think of a single mechanism able to reproduce all the details we have directly observed since physical boundary conditions favor or inhibit different generation mechanisms, like for instance, velocity-shear or parametric decay, depending on where we are in the heliosphere.

On the other hand, there are some aspects which we believe are at the basis of turbulence generation and evolution like: a) we do need non-linear interactions to develop the observed Kolmogorov-like spectrum; b) in order to have non-linear interactions we need to have inward modes and/or convected structures which the majority of the modes can interact with; c) outward and inward modes can be generated by different mechanisms like velocity shear or parametric decay; d) convected structures actively contribute to turbulent development of fluctuations and can be of solar origin or locally generated.

In particular, ecliptic observations have shown that what we call Alfvénic turbulence, mainly observed within high velocity streams, tends to evolve towards the more "standard" turbulence that we mainly observe within slow wind regions, i.e., a turbulence characterized by $e^{+} \sim e^{-}$, an excess of magnetic energy, and a Kolmogorov-like spectral slope. Moreover, the presence of a well established "background" spectrum already at short heliocentric distances and the low Alfvénicity of the fluctuations suggest that within slow wind turbulence is mainly due to convected structures frozen in the wind which may well be the remnants of turbulent processes already acting within the first layers of the solar corona. In addition, velocity shear, whenever present, seems to have a relevant role in driving turbulence evolution in low-latitude solar wind.

Polar observations performed by Ulysses, combined with previous results in the ecliptic, finally allowed to get a comprehensive view of the Alfvénic turbulence evolution in the 3D heliosphere, inside 5 AU. However, polar observations, when compared with results obtained in the ecliptic, do not appear as a dramatic break. In other words, the polar evolution is similar to that in the ecliptic, although slower. This is a middle course between the two opposite views (a non-relaxing turbulence, due to the lack of velocity shear, or a quick evolving turbulence, due to the large relative amplitude of fluctuations) which were popular before the Ulysses mission. The process driving the evolution of polar turbulence still is an open question although parametric decay might play some role. As a matter of fact, simulations of non-linear development of the parametric instability for large-amplitude, broadband Alfvénic fluctuations have shown that the final state resembles values of $\sigma_{\mathrm{c}}$ not far from solar wind observations, in a state in which the initial Alfvénic correlation is partially preserved. As already observed in the ecliptic, polar Alfvénic turbulence appears characterized by a predominance of outward fluctuations and magnetic fluctuations. As regards the outward fluctuations, their dominant character extends to large distances from the Sun. At low solar activity, with the polar wind filling a large fraction of the heliosphere, the outward fluctuations should play a relevant role in the heliospheric physics. Relatively to the imbalance in favor of the magnetic energy, it does not appear to go beyond an asymptotic value. Several ways to alter the balance between kinetic and magnetic energy have been proposed (e.g., 2D processes, propagation in a non-uniform medium, and effect of magnetic structures, among others). However, convincing arguments to account for the existence of such a limit have not yet been given, although promising results from numerical simulations seem to be able to qualitatively reproduce the final imbalance in favor of the magnetic energy.

Living Reviews in Solar Physics

http: //www. livingreviews . org/lrsp-2005-4 
Definitely, the relatively recent adoption of numerical methods able to highlight scaling laws features hidden to the usual spectral methods, allowed to disclose a new and promising way to analyze turbulent interplanetary fluctuations. Interplanetary space is now looked at as a natural wind tunnel where scaling properties of the solar wind can be studied on scales of the order of (or larger than) $10^{9}$ times than laboratory scales. Within this framework, intermittency represents an important topic in both theoretical and observational studies. Intermittency properties have been recovered via very promising models like the MHD GOY-model, and the nature of intermittent events has finally been disclosed thanks to new numerical techniques based on wavelet transforms. Moreover, similar techniques have allowed to tackle the problem of identifying either Kraichnan or Kolmogorov scaling although no conclusive and final analyses have been reported so far. In addition, recent studies on intermittency of magnetic field and velocity vector fluctuations, together with analogous analyses on magnitude fluctuations, contributed to sketch a scenario in which propagating stochastic Alfvénic fluctuations and convected structures, possibly flux tubes embedded in the wind, represent the main ingredients of interplanetary turbulence. The relative predominance of one or the other contribution would make the observed turbulence more or less intermittent. However, the fact that we can make measurements just in one point of this natural wind tunnel represented by the solar wind does not allow us to discriminate temporal from spatial phenomena. As a consequence, we do not know whether these convected structures are somehow connected to the complicated topology observed at the Sun surface or can be considered as by-product of chaotic developing phenomena. Comparative studies based on the intermittency phenomenon within fast and slow wind during the wind expansion would suggest a solar origin for these structures which would form a sort of turbulent background frozen in the wind. As a matter of fact, intermittency in the solar wind is not limited to the dissipation range of the spectrum but abundantly extends orders of magnitude away from dissipative scales, possibly into the inertial range which can be identified taking into account all the possible caveats related to this problem and briefly reported in this review. This fact introduces serious differences between hydrodynamic turbulence and solar wind MHD turbulence, and the same "intermittency" assumes a different intrinsic meaning when observed in interplanetary turbulence. In practice, coherent structures observed in the wind are at odds with filaments or vortexes observed in ordinary fluid turbulence since these last ones are dissipative structures continuously created and destroyed by turbulent motion.

As a final remark, we would like to point out that we tried to start writing a particular point of view on the turbulence in the solar wind. We apologize for the lack of some aspects of the phenomenon at hand which can be found in the existing literature. There are several topics which we did not discuss in this first version of our review like recent (non-shell) turbulence modeling, simulation of turbulence in the expanding solar wind, uses of turbulence in coronal heating models, multispacecraft observations, and the kinetic approach to the dissipation of turbulence. Fortunately, we are writing a Living Review paper and mistakes and/or omissions will be adequately fixed in the next version also with the help of all our colleagues whom we strongly encourage to send us comments and/or different points of view on particularly interesting topics which we have not yet taken into account or discussed properly. 


\section{Acknowledgments}

Writing a large review paper is not an easy task and it would not have been possible to accomplish this goal without having a good interaction with our colleagues, whom we have been working with in our Institutions. To this regard, we like to acknowledge the many discussions (more or less "heated") we had with and the many advices and comments we had from all of them, particularly from B. Bavassano and P. Veltri. We also like to acknowledge the use of plasma and magnetic field data from Helios spacecraft to freshly produce some of the figures shown in the present review. In particular, we like to thank H. Rosenbauer and R. Schwenn, PIs of the plasma experiment, and F. Mariani and N.F. Ness, PIs of the second magnetic experiment on board Helios. We thank A. Pouquet, H. Politano, and V. Antoni for the possibility we had to compare solar wind data with both high-resolution numerical simulations and laboratory plasmas. Finally, we ought special thanks to E. Marsch and S.K. Solanki for giving us the opportunity to write this review.

Living Reviews in Solar Physics

http: //www . livingreviews . org/lrsp-2005-4 


\section{Appendix A: Some Characteristic Solar Wind Parame- ters}

Although solar wind is a highly variable medium, it is possible to identify some characteristic values for its most common parameters. Since the wind is an expanding medium, we ought to choose one heliocentric distance to refer to and, usually, this distance is $1 \mathrm{AU}$. In the following we will provide different tables referring to several solar wind parameters, velocities, characteristic times, and lengths.

As it can be seen, the solar wind is a super-Alfvénic, collisionless plasma, and MHD turbulence can be investigated for frequencies smaller than $\sim 10^{-1} \mathrm{~Hz}$.

Table 6: Typical values of several solar wind parameters as measured by Helios 2 at 1 AU.

\begin{tabular}{lll}
\hline Wind Parameter & Slow wind & Fast wind \\
\hline number density & $\sim 15 \mathrm{~cm}^{-3}$ & $\sim 4 \mathrm{~cm}^{-3}$ \\
bulk velocity & $\sim 350 \mathrm{~km} \mathrm{~s}^{-1}$ & $\sim 600 \mathrm{~km} \mathrm{~s}^{-1}$ \\
proton temperature & $\sim 5 \cdot 10^{4} \mathrm{~K}$ & $\sim 2 \cdot 10^{5} \mathrm{~K}$ \\
electron temperature & $\sim 2 \cdot 10^{5} \mathrm{~K}$ & $\sim 1 \cdot 10^{5} \mathrm{~K}$ \\
$\alpha$-particles temperature & $\sim 2 \cdot 10^{5} \mathrm{~K}$ & $\sim 8 \cdot 10^{5} \mathrm{~K}$ \\
magnetic field & $\sim 6 \mathrm{nT}$ & $\sim 6 \mathrm{nT}$ \\
\hline
\end{tabular}

Table 7: Typical values of different speeds obtained at 1 AU. The Alfvén speed has been measured, while all the others have been obtained from the parameters reported in Table 6 .

\begin{tabular}{lll}
\hline Speed & Slow wind & Fast wind \\
\hline Alfvén & $\sim 30 \mathrm{~km} \mathrm{~s}^{-1}$ & $\sim 60 \mathrm{~km} \mathrm{~s}^{-1}$ \\
ion sound & $\sim 60 \mathrm{~km} \mathrm{~s}^{-1}$ & $\sim 60 \mathrm{~km} \mathrm{~s}^{-1}$ \\
proton thermal & $\sim 35 \mathrm{~km} \mathrm{~s}^{-1}$ & $\sim 70 \mathrm{~km} \mathrm{~s}^{-1}$ \\
electron thermal & $\sim 3000 \mathrm{~km} \mathrm{~s}^{-1}$ & $\sim 2000 \mathrm{~km} \mathrm{~s}^{-1}$
\end{tabular}

Table 8: Typical values of different frequencies at 1 AU. These values have been obtained from the parameters reported in Table 6.

\begin{tabular}{lll}
\hline Frequency & Slow wind & Fast wind \\
\hline proton cyclotron & $\sim 0.1 \mathrm{~Hz}$ & $\sim 0.1 \mathrm{~Hz}$ \\
electron cyclotron & $\sim 2 \cdot 10^{2} \mathrm{~Hz}$ & $\sim 2 \cdot 10^{2} \mathrm{~Hz}$ \\
plasma & $\sim 2 \cdot 10^{5} \mathrm{~Hz}$ & $\sim 1 \cdot 10^{5} \mathrm{~Hz}$ \\
proton-proton collision & $\sim 2 \cdot 10^{-6} \mathrm{~Hz}$ & $\sim 1 \cdot 10^{-7} \mathrm{~Hz}$ \\
\hline
\end{tabular}


Table 9: Typical values of different lengths at 1 AU plus the distance traveled by a proton before colliding with another proton. These values have been obtained from the parameters reported in Table 6.

\begin{tabular}{lll}
\hline Length & Slow wind & Fast wind \\
\hline Debye & $\sim 4 \mathrm{~m}$ & $\sim 15 \mathrm{~m}$ \\
proton gyroradius & $\sim 130 \mathrm{~km}$ & $\sim 260 \mathrm{~km}$ \\
electron gyroradius & $\sim 2 \mathrm{~km}$ & $\sim 1.3 \mathrm{~km}$ \\
distance between 2 proton collisions & $\sim 1.2 \mathrm{AU}$ & $\sim 40 \mathrm{AU}$ \\
\hline
\end{tabular}

\section{Appendix B: Tools to Analyze MHD Turbulence in Space Plasmas}

No matter where we are in the solar wind, short scale data always look rather random.

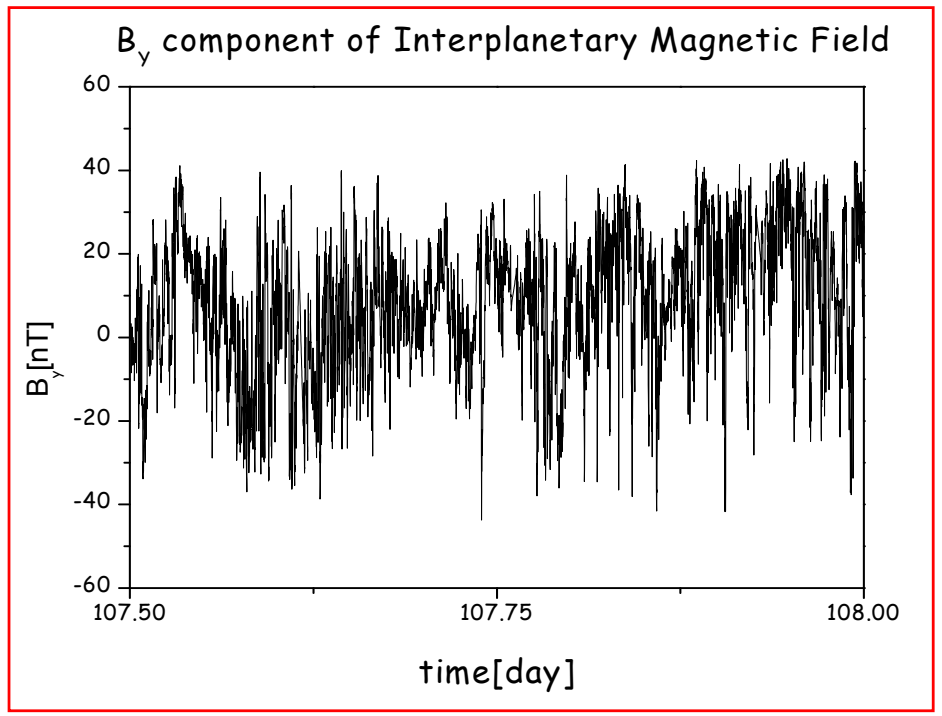

Figure 97: $B_{Y}$ component of the IMF recorded within a high velocity stream.

This aspect introduces the problem of determining the time stationarity of the dataset. The concept of stationarity is related to ensembled averaged properties of a random process. The random process is the collection of the $N$ samples $x(t)$, it is called ensemble and indicated as $\{x(t)\}$.

Properties of a random process $\{x(t)\}$ can be described by averaging over the collection of all the $N$ possible sample functions $x(t)$ generated by the process. So, chosen a begin time $t_{1}$, we can define the mean value $\mu_{x}$ and the autocorrelation function $R_{x}$, i.e., the first and the joint moment:

$$
\mu_{x}\left(t_{1}\right)=\lim _{N \longrightarrow \infty} \sum_{k=1}^{N} x_{k}\left(t_{1}\right),
$$

Living Reviews in Solar Physics

http: //www . livingreviews . org/lrsp-2005-4 


$$
R_{x}\left(t_{1}, t_{1}+\tau\right)=\lim _{N \longrightarrow \infty} \sum_{k=1}^{N} x_{k}\left(t_{1}\right) x_{k}\left(t_{1}+\tau\right)
$$

In case $\mu_{x}\left(t_{1}\right)$ and $R_{x}\left(t_{1}, t_{1}+\tau\right)$ do not vary as time $t_{1}$ varies, the sample function $x(t)$ is said to be weakly stationary, i.e.,

$$
\begin{gathered}
\mu_{x}\left(t_{1}\right)=\mu_{x}, \\
R_{x}\left(t_{1}, t_{1}+\tau\right)=R_{x}(\tau) .
\end{gathered}
$$

Strong stationarity would require all the moments and joint moments to be time independent. However, if $x(t)$ is normally distributed, the concept of weak stationarity naturally extends to strong stationarity.

Generally, it is possible to describe the properties of $\{x(t)\}$ simply computing time-averages over just one $x(t)$. If the random process is stationary and $\mu_{x}(k)$ and $R_{x}(\tau, k)$ do not vary when computed over different sample functions, the process is said ergodic. This is a great advantage for data analysts, especially for those who deals with data from s/c, since it means that properties of stationary random phenomena can be properly measured from a single time history. In other words, we can write:

$$
\begin{aligned}
\mu_{x}(k) & =\mu_{x}, \\
R_{x}(\tau, k) & =R_{x}(\tau) .
\end{aligned}
$$

Thus, the concept of stationarity, which is related to ensembled averaged properties, can now be transferred to single time history records whenever properties computed over a short time interval do not vary from one interval to the next more than the variation expected for normal dispersion.

Fortunately, Matthaeus and Goldstein (1982a) established that interplanetary magnetic field often behaves as a stationary and ergodic function of time, if coherent and organized structures are not included in the dataset. Actually, they proved the weak stationarity of the data, i.e., the stationarity of the average and two-point correlation function. In particular, they found that the average and the autocorrelation function computed within a subinterval would converge to the values estimated from the whole interval after a few correlation times $t_{\mathrm{c}}$.

If our time series approximates a Markov process (a process whose relation to the past does not extend beyond the immediately preceding observation), its autocorrelation function can be shown (Doob, 1953) to approximate a simple exponential:

$$
R(t)=R(0) e^{-\frac{t}{t_{c}}}
$$

from which, we obtain the definition given by Batchelor (1970):

$$
t_{\mathrm{c}}=\int_{0}^{\infty} \frac{R(t)}{R(0)} d t
$$

Just to have an idea of the correlation time of magnetic field fluctuations, we show in Figure 98 magnetic field correlation time computed at 1 AU using Voyager's 2 data.

In this case, using the above definition, $t_{\mathrm{c}} \simeq 3.2 \cdot 10^{3} \mathrm{~s}$.

\subsection{Statistical description of MHD turbulence}

When an MHD fluid is turbulent, it is impossible to know the detailed behavior of velocity field $v(x, t)$ and magnetic field $b(x, t)$, and the only description available is the statistical one. Very useful is the knowledge of the invariants of the ideal equations of motion for which the dissipative terms $\mu \nabla^{2} \mathbf{b}$ and $\nu \nabla^{2} \mathbf{v}$ are equal to zero because the magnetic resistivity $\mu$ and the viscosity $\nu$ are both equal to zero. Following Frisch et al. (1975) there are three quadratic invariants of the 


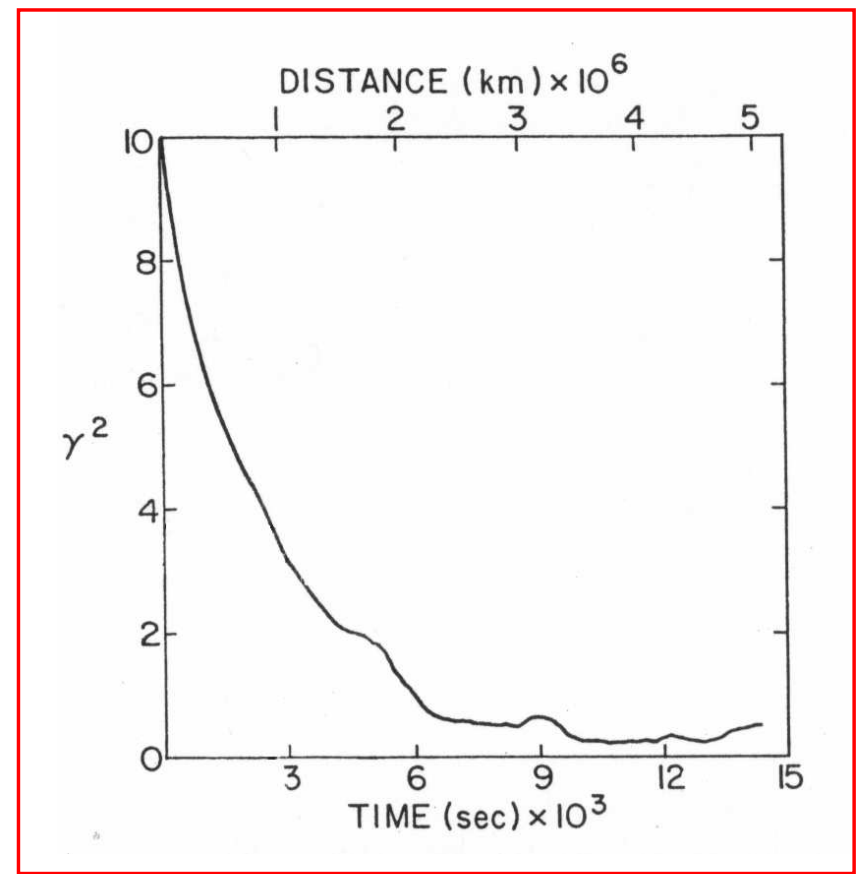

Figure 98: Magnetic field auto-correlation function at $1 \mathrm{AU}$ (adopted from Matthaeus and Goldstein, 1982b, (C) 1982 American Geophysical Union, reproduced by permission of American Geophysical Union). 
ideal system which can be used to describe MHD turbulence: total energy $E$, cross-helicity $H_{\mathrm{c}}$, and magnetic helicity $H_{\mathrm{m}}$. The above quantities are defined as follows:

$$
\begin{gathered}
E=\frac{1}{2}<v^{2}+b^{2}>, \\
H_{\mathrm{c}}=<\mathbf{v} \cdot \mathbf{b}>, \\
H_{\mathrm{m}}=<\mathbf{A} \cdot \mathbf{B}>,
\end{gathered}
$$

where $\mathbf{v}$ and $\mathbf{b}$ are the fluctuations of velocity and magnetic field, this last one expressed in Alfvén units $\left(b \longrightarrow \frac{b}{\sqrt{4 \pi \rho}}\right)$, and $\mathbf{A}$ is the vector potential so that $\mathbf{B}=\nabla \times \mathbf{A}$. The integrals of these quantities over the entire plasma containing regions are the invariants of the ideal MHD equations:

$$
\begin{aligned}
& E=\frac{1}{2} \int\left(v^{2}+b^{2}\right) d^{3} x, \\
& H_{\mathrm{c}}=\frac{1}{2} \int(\mathbf{v} \cdot \mathbf{b}) d^{3} x, \\
& H_{\mathrm{m}}=\int(\mathbf{A} \cdot \mathbf{B}) d^{3} x,
\end{aligned}
$$

In particular, in order to describe the degree of correlation between $v$ and $b$, it is convenient to use the normalized cross-helicity $\sigma_{\mathrm{c}}$ :

$$
\sigma_{\mathrm{c}}=\frac{2 H_{\mathrm{c}}}{E}
$$

since this quantity simply varies between +1 and -1 .

\subsection{Spectra of the invariants in homogeneous turbulence}

Statistical information about the state of a turbulent fluid is contained in the $n$-point correlation function of the fluctuating fields. In homogeneous turbulence these correlations are invariant under arbitrary translation or rotation of the experimental apparatus. We can define the magnetic field auto-correlation matrix

$$
R_{i j}^{b}(r)=<b_{i}(x) b_{j}(x+r)>,
$$

the velocity auto-correlation matrix

$$
R_{i j}^{v}(r)=<v_{i}(x) v_{j}(x+r)>,
$$

and the cross-correlation matrix

$$
R_{i j}^{v b}(r)=\frac{1}{2}<v_{i}(x) b_{j}(x+r)+b_{i}(x) v_{j}(x+r)>.
$$

At this point, we can construct the spectral matrix in terms of Fourier transform of $R_{i j}$

$$
\begin{aligned}
& S_{i j}^{b}(\mathbf{k})=\frac{1}{2 \pi} \int R_{i j}^{b}(\mathbf{r}) e^{-i \mathbf{k} \cdot \mathbf{r}} d^{3} r, \\
& S_{i j}^{v}(\mathbf{k})=\frac{1}{2 \pi} \int R_{i j}^{v}(\mathbf{r}) e^{-i \mathbf{k} \cdot \mathbf{r}} d^{3} r, \\
& S_{i j}^{v b}(\mathbf{k})=\frac{1}{2 \pi} \int R_{i j}^{v b}(\mathbf{r}) e^{-i \mathbf{k} \cdot \mathbf{r}} d^{3} r .
\end{aligned}
$$


However, in space experiments, especially in the solar wind, data from only a single spacecraft are available. This provides values of $R_{i j}^{b}, R_{i j}^{v}$, and $R_{i j}^{v b}$, for separations along a single direction r. In this situation, only reduced (i.e., one-dimensional) spectra can be measured. If $\mathbf{r}_{1}$ is the direction of co-linear separations, we may only determine $R_{i j}\left(r_{1}, 0,0\right)$ and, as a consequence, the Fourier transform on $R_{i j}$ yields the reduced spectral matrix

$$
S_{i j}^{r}\left(\mathbf{k}_{\mathbf{1}}\right)=\frac{1}{2 \pi} \int R_{i j}\left(r_{1}, 0,0\right) e^{-i \mathbf{k}_{\mathbf{1}} \cdot \mathbf{r}_{\mathbf{1}}} d r_{1}=\int S_{i j}\left(k_{1}, k_{2}, k_{3}\right) d k_{2} d k_{3}
$$

Then, we define $H_{m}^{r}, H_{c}^{r}$, and $E^{r}=E_{b}^{r}+E_{v}^{r}$ as the reduced spectra of the invariants, depending only on the wave number $k_{1}$. Complete information about Sij might be lost when computing its reduced version since we integrate over the two transverse k. However, for isotropic symmetry no information is lost performing the transverse wave number integrals (Batchelor, 1970). That is, the same spectral information is obtained along any given direction.

Coming back to the ideal invariants, now we have to deal with the problem of how to extract information about $H_{\mathrm{m}}$ from $R_{i j}(r)$. We know that the Fourier transform of a real, homogeneous matrix $R_{i j}(r)$ is an Hermitian form $S_{i j}$, i.e., $S=\tilde{S}^{*} \longrightarrow s_{i j}=s_{j i}^{*}$, and that any square matrix $A$ can be decomposed into a symmetric and an antisymmetric part, $A^{s}$ and $A^{a}$ :

$$
A=A^{s}+A^{a}
$$

where

$$
\begin{aligned}
A^{s} & =\frac{1}{2}(A+\tilde{A}), \\
A^{a} & =\frac{1}{2}(A-\tilde{A}) .
\end{aligned}
$$

Since the Hermitian form implies that

$$
S=\tilde{S}^{*} \longrightarrow s_{i j}=s_{j i}^{*}
$$

it follows that

$$
S^{s}=\frac{1}{2}(S+\tilde{S})=\frac{1}{2}\left(S_{i j}+S_{j i}\right)=\text { real }
$$

and

$$
S^{a}=\frac{1}{2}(S-\tilde{S})=\frac{1}{2}\left(S_{i j}-S_{j i}\right)=\text { imaginary }
$$

It has been shown (Batchelor, 1970; Matthaeus and Goldstein, 1982b; Montgomery, 1983) that, while the trace of the symmetric part of the spectral matrix accounts for the magnetic energy, the imaginary part of the spectral matrix accounts for the magnetic helicity. In particular, Matthaeus and Goldstein (1982b) showed that

$$
H_{\mathrm{m}}^{r}\left(k_{1}\right)=2 \operatorname{Im} S_{23}^{r}\left(k_{1}\right) / k_{1}
$$

where $H_{\mathrm{m}}$ has been integrated over the two transverse components

$$
\int I m S_{23}(\mathbf{k}) d k_{2} d k_{3}=\frac{k_{1}}{2} \int H_{\mathrm{m}}(\mathbf{k}) d k_{2} d k_{3} .
$$

In practice, if co-linear measurements are made along the $\mathrm{X}$ direction, the reduced magnetic helicity spectrum is given by:

$$
H_{\mathrm{m}}^{r}\left(k_{1}\right)=2 \operatorname{Im} S_{23}^{r}\left(k_{1}\right) / k_{1}=2 \operatorname{Im}\left(Y Z^{*}\right) / k_{1},
$$

Living Reviews in Solar Physics

http: //www . livingreviews . org/lrsp-2005-4 
where $Y$ and $Z$ are the Fourier transforms of $B_{y}$ and $B_{z}$ components, respectively.

$H_{\mathrm{m}}$ can be interpreted as a measure of the correlation between the two transverse components, being one of them shifted by $90^{\circ}$ in phase at frequency $f$. This parameter gives also an estimate of how magnetic field lines are knotted with each other. $H_{\mathrm{m}}$ can assume positive and negative values depending on the sense of rotation of the correlation between the two transverse components.

However, another parameter, which is a combination of $H_{\mathrm{m}}$ and $E_{b}$, is usually used in place of $H_{\mathrm{m}}$ alone. This parameter is the normalized magnetic helicity

$$
\sigma_{\mathrm{m}}(k)=k H_{\mathrm{m}}(k) / E_{b}(k),
$$

where $E_{b}$ is the magnetic spectral power density and $\sigma_{\mathrm{m}}$ varies between +1 and -1 .

\subsubsection{Coherence and phase}

Since the cross-correlation function is not necessarily an even function, the cross-spectral density function is generally a complex number:

$$
W_{x y}(f)=C_{x y}(f)+j Q_{x y}(f),
$$

where the real part $C_{x y}(f)$ is the coincident spectral density function, and the imaginary part $Q_{x y}(f)$ is the quadrature spectral density function (Bendat and Piersol, 1971). While $C_{x y}(f)$ can be thought of as the average value of the product $x(t) y(t)$ within a narrow frequency band $(f, f+\delta f), Q_{x y}(f)$ is similarly defined but one of the components is shifted in time sufficiently to produce a phase shift of $90^{\circ}$ at frequency $f$.

In polar notation

$$
W_{x y}(f)=\left|W_{x y}(f)\right| e^{-j \theta_{x y}(f)} .
$$

In particular,

$$
\left|W_{x y}(f)\right|=\sqrt{C_{x y}^{2}(f)+Q_{x y}^{2}(f)},
$$

and the phase between $C$ and $Q$ is given by

$$
\theta_{x y}(f)=\arctan \frac{Q_{x y}(f)}{C_{x y}(f)} .
$$

Moreover,

$$
\left|W_{x y}(f)\right|^{2} \leq W_{x}(f) W_{y}(f),
$$

so that the following relation holds

$$
\gamma_{x y}^{2}(f)=\frac{\left|W_{x y}(f)\right|^{2}}{W_{x}(f) W_{y}(f)} \leq 1 .
$$

This function $\gamma_{x y}^{2}(f)$, called coherence, estimates the correlation between $x(t)$ and $y(t)$ for a given frequency $f$. Just to give an example, for an Alfvén wave at frequency $f$ whose $\mathbf{k}$ vector is outwardly oriented as the interplanetary magnetic field, we expect to find $\theta_{v b}(f)=180^{\circ}$ and $\gamma_{v b}^{2}(f)=1$, where the indexes $v$ and $b$ refer to the magnetic field and velocity field fluctuations.

\subsection{Introducing the Elsässer variables}

The Alfvénic character of turbulence suggests to use the Elsässer variables to better describe the inward and outward contributions to turbulence. Following Elsässer (1950), Dobrowolny et al. 
(1980b), Goldstein et al. (1986), Grappin et al. (1989), Marsch and Tu (1989), Tu and Marsch (1990a), and Tu et al. (1989c), Elsässer variables are defined as

$$
\mathbf{z}^{ \pm}=\mathbf{v} \pm \frac{\mathbf{b}}{\sqrt{4 \pi \rho}}
$$

where $\mathbf{v}$ and $\mathbf{b}$ are the proton velocity and the magnetic field measured in the s/c reference frame, which can be looked at as an inertial reference frame. The sign in front of $\mathbf{b}$, in Equation (79), is decided by $\operatorname{sign}\left[-\mathbf{k} \cdot \mathbf{B}_{0}\right]$. In other words, for an outward directed mean field $\mathbf{B}_{0}$, a negative correlation would indicate an outward directed wave vector $\mathbf{k}$ and vice-versa. However, it is more convenient to define the Elsässers variables in such a way that $z^{+}$always refers to waves going outward and $z^{-}$to waves going inward. In order to do so, the background magnetic field $\mathbf{B}_{0}$ is artificially rotated by $180^{\circ}$ every time it points away from the Sun, in other words, magnetic sectors are rectified (Roberts et al., 1987a,b).

\subsubsection{Definitions and conservation laws}

If we express $\mathbf{b}$ in Alfvén units, that is we normalize it by $\sqrt{4 \pi \rho}$ we can use the following handy formulas relative to definitions of fields and second order moments. Fields:

$$
\begin{gathered}
\mathbf{z}^{ \pm}=\mathbf{v} \pm \mathbf{b}, \\
\mathbf{v}=\frac{1}{2}\left(\mathbf{z}^{+}+\mathbf{z}^{-}\right), \\
\mathbf{b}=\frac{1}{2}\left(\mathbf{z}^{+}-\mathbf{z}^{-}\right) .
\end{gathered}
$$

Second order moments:

$$
\begin{gathered}
\left.z^{+} \text {and } z^{-} \text {energies } \longrightarrow e^{ \pm}=\frac{1}{2}<\left(z^{ \pm}\right)^{2}\right\rangle, \\
\text { kinetic energy } \longrightarrow e^{v}=\frac{1}{2}<v^{2}> \\
\text { magnetic energy } \longrightarrow e^{b}=\frac{1}{2}<b^{2}> \\
\text { total energy } \longrightarrow e=e^{v}+e^{b}, \\
\text { residual energy } \longrightarrow e^{r}=e^{v}-e^{b}, \\
\text { cross-helicity } \longrightarrow e^{c}=\frac{1}{2}<\mathbf{v} \cdot \mathbf{b}>
\end{gathered}
$$

Normalized quantities:

$$
\begin{gathered}
\text { normalized cross-helicity } \longrightarrow \sigma_{\mathrm{c}}=\frac{e^{+}-e^{-}}{e^{+}+e^{-}}=\frac{2 e^{c}}{e^{v}+e^{b}}, \\
\text { normalized residual-energy } \longrightarrow \sigma_{r}=\frac{e^{v}-e^{b}}{e^{v}+e^{b}}=\frac{2 e^{r}}{e^{+}+e^{-}}, \\
\text {Alfvén ratio } \longrightarrow r_{\mathrm{A}}=\frac{e^{v}}{e^{b}}=\frac{1+\sigma_{r}}{1-\sigma_{r}}, \\
\text { Elsässer ratio } \longrightarrow r_{\mathrm{E}}=\frac{e^{-}}{e^{+}}=\frac{1-\sigma_{\mathrm{c}}}{1+\sigma_{\mathrm{c}}} .
\end{gathered}
$$

We expect an Alfvèn wave to satisfy the following relations:

Living Reviews in Solar Physics

http: //www . livingreviews . org/lrsp-2005-4 
Table 10: Expected values for Alfvèn ratio $r_{\mathrm{A}}$, normalized cross-helicity $\sigma_{\mathrm{c}}$, and normalized residual energy $\sigma_{\mathrm{r}}$ for a pure Alfvèn wave outward or inward oriented.

\begin{tabular}{ccc}
\hline Parameter & Definition & Expected Value \\
\hline$r_{\mathrm{A}}$ & $e^{V} / e^{B}$ & 1 \\
$\sigma_{\mathrm{c}}$ & $\left(e^{+}-e^{-}\right) /\left(e^{+}+e^{-}\right)$ & \pm 1 \\
$\sigma_{\mathrm{r}}$ & $\left(e^{V}-e^{B}\right) /\left(e^{V}+e^{B}\right)$ & 0 \\
\hline
\end{tabular}

\subsubsection{Spectral analysis using Elsässer variables}

A spectral analysis of interplanetary data can be performed using $z^{+}$and $z^{-}$fields. Following Tu and Marsch (1995a) the energy spectrum associated with these two variables can be defined in the following way:

$$
e_{j}^{ \pm}\left(f_{k}\right)=\frac{2 \delta T}{n} \delta z_{j, k}^{ \pm}\left(\delta z_{j, k}^{ \pm}\right)^{*},
$$

where $\delta z_{j, k}^{ \pm}$are the Fourier coefficients of the j-component among $x, y$, and $z, n$ is the number of data points, $\delta T$ is the sampling time, and $f_{k}=k / n \delta T$, with $k=0,1,2, \ldots, n / 2$ is the $k$-th frequency. The total energy associated with the two Alfvèn modes will be the sum of the energy of the three components, i.e.,

$$
e^{ \pm}\left(f_{k}\right)=\sum_{j=x, y, z} e_{j}^{ \pm}\left(f_{k}\right)
$$

Obviously, using Equations $(93,94)$, we can redefine in the frequency domain all the parameters introduced in the previous section. 


\section{Appendix C: Wavelets as a Tool to Study Intermittency}

Following Farge et al. (1990) and Farge (1992), intermittent events can be viewed as localized zones of fluid where phase correlation exists, in some sense coherent structures. These structures, which dominate the statistic of small scales, occur as isolated events with a typical lifetime which is greater than that of stochastic fluctuations surrounding them. Structures continuously appear and disappear, apparently in a random fashion, at some random location of fluid, and they carry most of the flow energy. In this framework, intermittency can be considered as the result of the occurrence of coherent (non-Gaussian) structures at all scales, within the sea of stochastic Gaussian fluctuations.

It follows that, since these structures are well localized in spatial scale and time, it would be advisable to analyze them using wavelets filter instead of the usual Fourier transform. Unlike the Fourier basis, wavelets allow a decomposition both in time and frequency (or space and scale). The wavelet transform $W\{f(t)\}$ of a function $f(t)$ consists of the projection of $f(t)$ on a wavelet basis to obtain wavelet coefficients $w(\tau, t)$. These coefficients are obtained through a convolution between the analyzed function and a shifted and scaled version of an optional wavelet base

$$
w(\tau, t)=\int f\left(t^{\prime}\right) \frac{1}{\sqrt{\tau}} \Psi\left(\frac{t-t^{\prime}}{\tau}\right) d t^{\prime},
$$

where the wavelet function

$$
\Psi_{t^{\prime}, \tau}(t)=\frac{1}{\sqrt{\tau}} \Psi\left(\frac{t-t^{\prime}}{\tau}\right)
$$

has zero mean and compact support. Some examples of translated and scaled version of this function for a particular wavelet called "charro", because its profile resembles the Mexican hat "El Charro", are given in Figure 99, and the analytical expression for this wavelet is

$$
\Psi_{t^{\prime}, \tau}(t)=\frac{1}{\sqrt{\tau}}\left[\left(1-\left(\frac{t-t^{\prime}}{\tau}\right)^{2}\right) \exp \left(-\frac{1}{2}\left(\frac{t-t^{\prime}}{\tau}\right)^{2}\right)\right] .
$$

Since the Parceval's theorem exists, the square modulus $|w(\tau, t)|^{2}$ represents the energy content of fluctuations $f(t+\tau)-f(t)$ at the scale $\tau$ at position $t$.

In analyzing intermittent structures it is useful to introduce a measure of local intermittency, as for example the Local Intermittency Measure (LIM) introduced by Farge (see, e.g., Farge et al., 1990; Farge, 1992)

$$
\operatorname{LIM}=\frac{|w(\tau, t)|^{2}}{\left\langle|w(\tau, t)|^{2}\right\rangle_{t}}
$$

(averages are made over all positions at a given scale $\tau$ ). The quantity from Equation (96) represents the energy content of fluctuations at a given scale with respect to the standard deviation of fluctuations at that scale. The whole set of wavelets coefficients can then be split in two sets: a set which corresponds to "Gaussian" fluctuations $w_{g}(\tau, t)$, and a set which corresponds to "structure" fluctuations $w_{s}(\tau, t)$, that is, the whole set of coefficients $w(\tau, t)=w_{g}(\tau, t) \oplus w_{s}(\tau, t)$ (the symbol $\oplus$ stands here for the union of disjoint sets). A coefficient at a given scale and position will belong to a structure or to the Gaussian background according whether LIM will be respectively greater or lesser than a threshold value. An inverse wavelets transform performed separately on both sets, namely $f_{g}(t)=W^{-1}\left\{w_{g}(\tau, t)\right\}$ and $f_{s}(t)=W^{-1}\left\{w_{s}(\tau, t)\right\}$, gives two separate fields: a field $f_{g}(t)$ where the Gaussian background is collected, and the field $f_{s}(t)$ where only the non-Gaussian fluctuations of the original turbulent flow are taken into account. Looking at the field $f_{s}(t)$ one can investigate the spatial behavior of structures generating intermittency. The Haar basis have

Living Reviews in Solar Physics

http: //www. livingreviews . org/lrsp-2005-4 


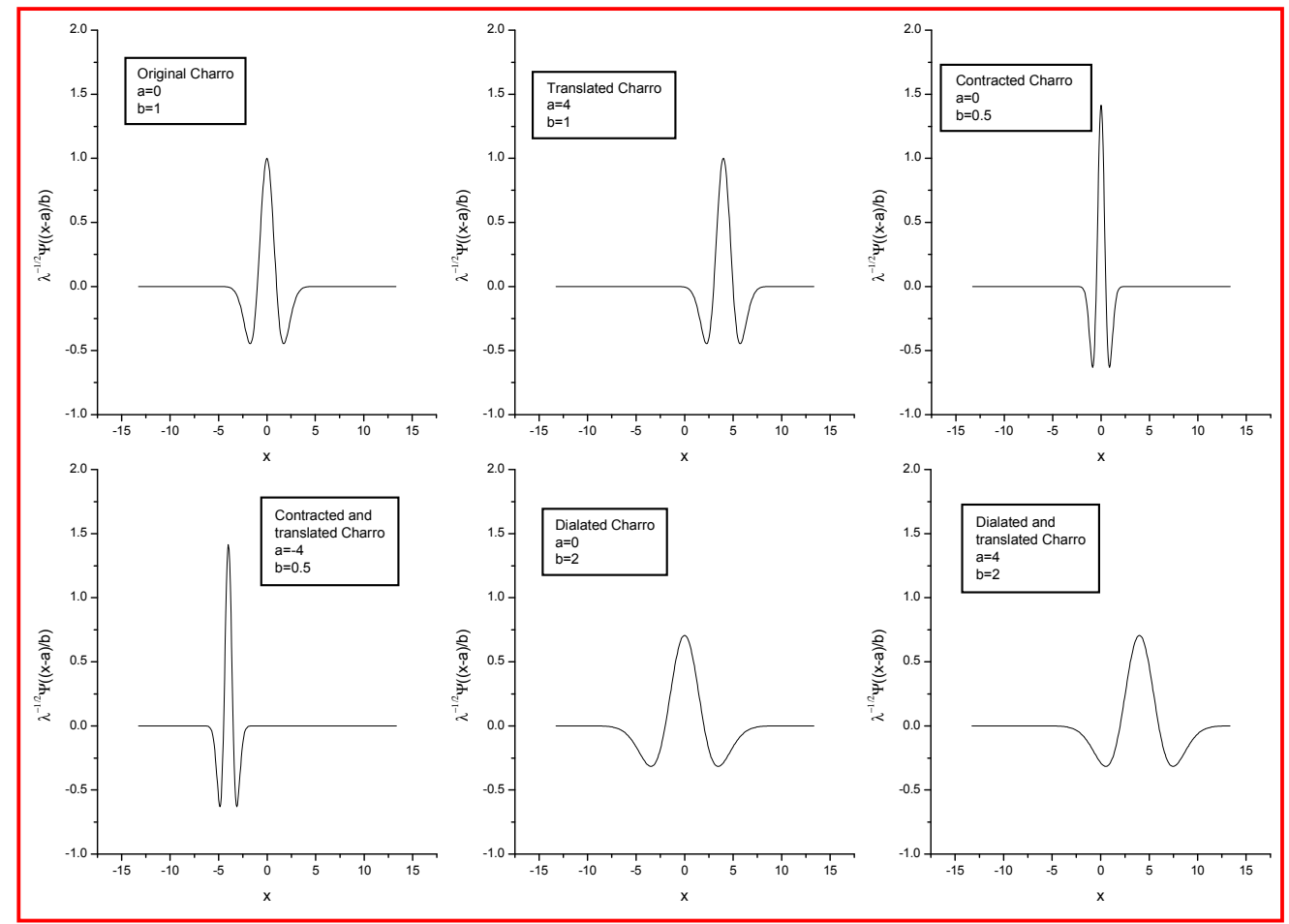

Figure 99: Some examples of Mexican Hat wavelet, for different values of the parameters $\tau$ and $t^{\prime}$. 
been applied to time series of thirteen months of velocity and magnetic data from ISEE space experiment for the first time by Veltri and Mangeney (1999b).

In our analyses we adopted a recursive method (Bianchini et al., 1999; Bruno et al., 1999a) similar to the one introduced by Onorato et al. (2000) to study experimental turbulent jet flows. The method consists in eliminating, for each scale, those events which cause LIM to exceed a given threshold. Subsequently, the flatness value for each scale is checked and, in case this value exceeds the value of 3 (characteristic of a Gaussian distribution), the threshold is lowered, new events are eliminated and a new flatness is computed. The process is iterated until the flatness is equal to 3 , or reaches some constant value, for each scale of the wavelet decomposition. This process is usually accomplished eliminating only a few percent of the wavelet coefficients for each scale, and this percentage reduces moving from small to large scales.

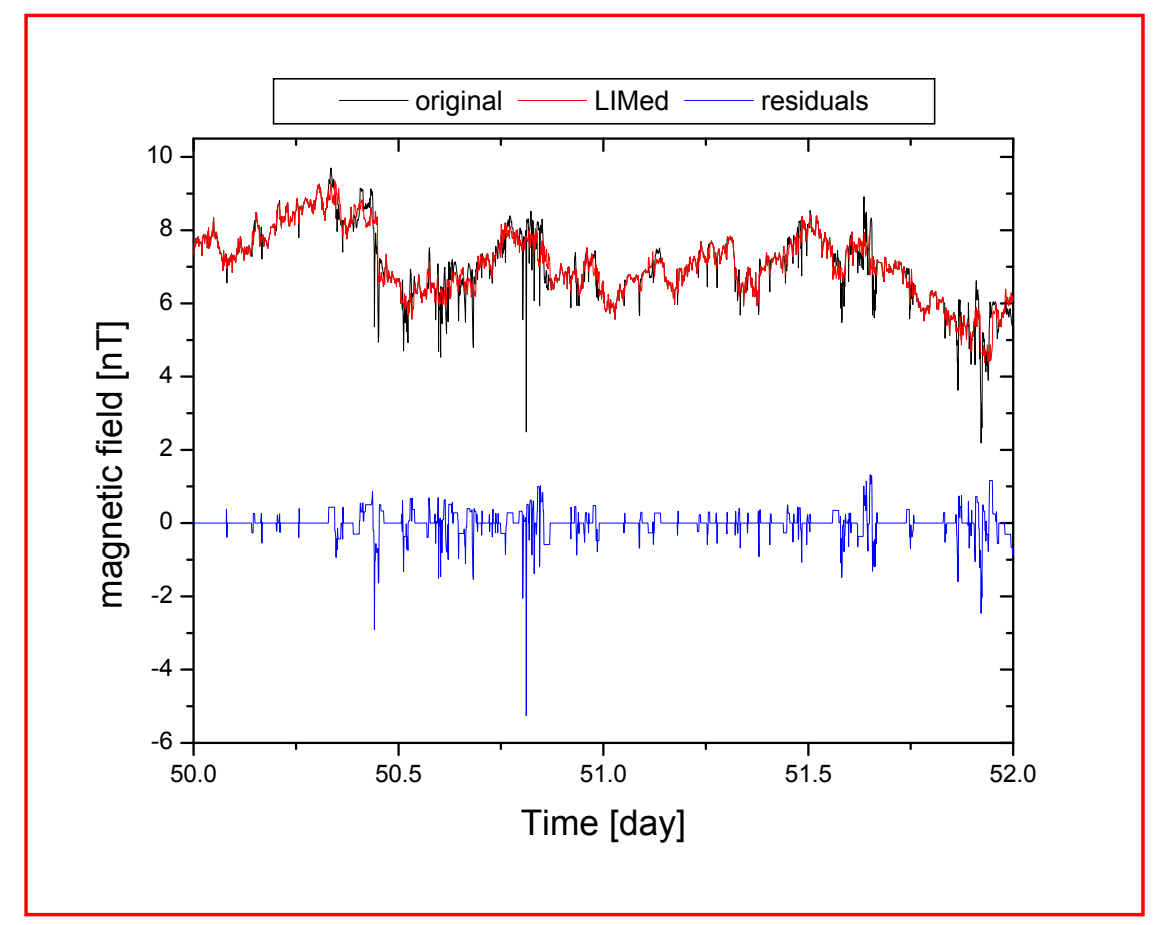

Figure 100: The black curve indicates the original time series, the red one refers to the LIMed data, and the blue one shows the difference between these two curves.

The black curve in Figure 100 shows the original profile of the magnetic field intensity observed by Helios 2 between day 50 and 52 within a highly velocity stream at $0.9 \mathrm{AU}$. The overlapped red profile refers to the same time series after intermittent events have been removed using the LIM method. Most of the peaks, present in the original time series, are not longer present in the LIMed curve. The intermittent component that has been removed can be observed as the blue curve centered around zero.

Living Reviews in Solar Physics

http://www. livingreviews .org/lrsp-2005-4 


\section{Appendix D: Reference Systems}

Interplanetary magnetic field and plasma data are provided, usually, in two main reference systems: RTN and SE.

The RTN system (see top part of Figure 101) has the $R$ axis along the radial direction, positive from the Sun to the s/c, the $T$ component perpendicular to the plane formed by the rotation axis of the Sun $\Omega$ and the radial direction, i.e., $T=\Omega \times R$, and the $N$ component resulting from the vector product $N=R \times T$.

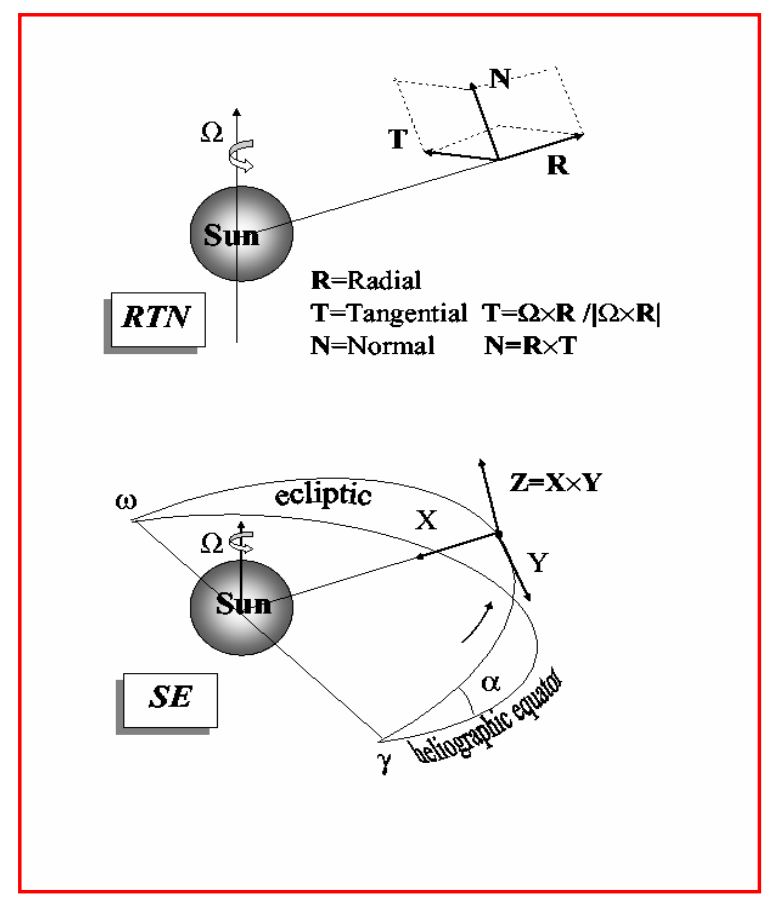

Figure 101: The top reference system is the RTN while the one at the bottom is the Solar Ecliptic reference system. This last one is shown in the configuration used for Helios magnetic field data, with the $\mathrm{X}$ axis positive towards the Sun.

The Solar Ecliptic reference system SE, is shown (see bottom part of Figure 101) in the configuration used for Helios magnetic field data, i.e., s/c centered, with the $X$ axis positive towards the Sun, and the $Y$ axis lying in the ecliptic plane and oriented opposite to the orbital motion. The third component $Z$ is defined as $Z=X \times Y$. However, solar wind velocity is given in the Sun-centered SE system, which is obtained from the previous one after a rotation of $180^{\circ}$ around the $Z$ axis.

Sometimes, studies are more meaningful if they are performed in particular reference systems which result to be rotated with respect to the usual systems, in which the data are provided in the data centers, for example RTN or SE. Here we will recall just two reference systems commonly used in data analysis.

Living Reviews in Solar Physics http://www . livingreviews . org/lrsp-2005-4 


\subsection{Minimum variance reference system}

The minimum variance reference system, i.e., a reference system with one of its axes aligned with a direction along whit the field has the smallest fluctuations (Sonnerup and Cahill, 1967). This method provides information on the spatial distribution of the fluctuations of a given vector.

Given a generic field $\mathrm{B}(\mathrm{x}, \mathrm{y}, \mathrm{z})$, the variance of its components is

$$
<B_{x}^{2}>-<B_{x}>^{2} ;<B_{y}^{2}>-<B_{y}>^{2} ;<B_{z}^{2}>-<B_{z}>^{2} .
$$

Similarly, the variance of B along the direction $S$ would be given by

$$
V_{S}=<B_{S}^{2}>-<B_{S}>^{2} .
$$

Let us assume, for sake of simplicity, that all the three components of $B$ fluctuate around zero, then

$$
<B_{x}>=<B_{y}>=<B_{z}>=0 \Longrightarrow<B_{S}>=\mathbf{x}<B_{x}>+\mathbf{y}<B_{y}>+\mathbf{z}<B_{z}>=0 .
$$

Then, the variance $V_{S}$ can be written as

$$
\begin{aligned}
V_{S}=<B_{S}^{2}>=x^{2} & <B_{x}^{2}>+y^{2}<B_{y}^{2}>+z^{2}<B_{z}^{2}>+2 \mathbf{x y}<B_{x} B_{y}>+ \\
& +2 \mathbf{x z}<B_{x} B_{z}>+2 \mathbf{y z}<B_{y} B_{z}>,
\end{aligned}
$$

which can be written (omitting the sign of average $<>$ ) as

$$
V_{S}=\mathbf{x}\left(\mathbf{x} B_{x}^{2}+\mathbf{y} B_{x} B_{y}+\mathbf{z} B_{x} B_{z}\right)+\mathbf{y}\left(\mathbf{y} B_{y}^{2}+\mathbf{x} B_{x} B_{y}+\mathbf{z} B_{y} B_{z}\right)+\mathbf{z}\left(\mathbf{z} B_{z}^{2}+\mathbf{x} B_{x} B_{z}+\mathbf{y} B_{y} B_{z}\right) .
$$

This expression can be interpreted as a scalar product between a vector $\mathbf{S}(x, y, z)$ and another vector whose components are the terms in parentheses. Moreover, these last ones can be expressed as a product between a matrix $M$ built with the terms $B_{x}^{2}, B_{y}^{2}, B_{z}^{2}, B_{x} B_{y}, B_{x} B_{z}, B_{y} B_{z}$, and a vector $\mathbf{S}(x, y, z)$. Thus,

$$
V_{S}=(S, M S)
$$

where

$$
S \equiv\left(\begin{array}{l}
x \\
y \\
z
\end{array}\right)
$$

and

$$
M \equiv\left(\begin{array}{ccc}
B_{x}{ }^{2} & B_{x} B_{y} & B_{x} B_{z} \\
B_{x} B_{y} & B_{y}{ }^{2} & B_{y} B_{z} \\
B_{x} B_{z} & B_{y} B_{z} & B_{z}{ }^{2}
\end{array}\right) .
$$

At this point, $M$ is a symmetric matrix and is the matrix of the quadratic form $V_{S}$ which, in turn, is defined positive since it represents a variance. It is possible to determine a new reference system $[x, y, z]$ such that the quadratic form $V_{S}$ does not contain mix terms, i.e.,

$$
V_{S}=x^{\prime 2}{B_{x}^{\prime}}^{2}+y^{\prime 2}{B_{y}^{\prime 2}}^{2}+z^{\prime 2}{B_{z}^{\prime}}^{2} .
$$

Thus, the problem reduces to compute the eigenvalues $\lambda_{i}$ and eigenvectors $\tilde{V}_{i}$ of the matrix $M$. The eigenvectors represent the axes of the new reference system, the eigenvalues indicate the variance along these axes as shown in Figure 102.

At this point, since we know the components of unit vectors of the new reference system referred to the old reference system, we can easily rotate any vector, defined in the old reference system, into the new one.

Living Reviews in Solar Physics

http: //www . livingreviews . org/lrsp-2005-4 


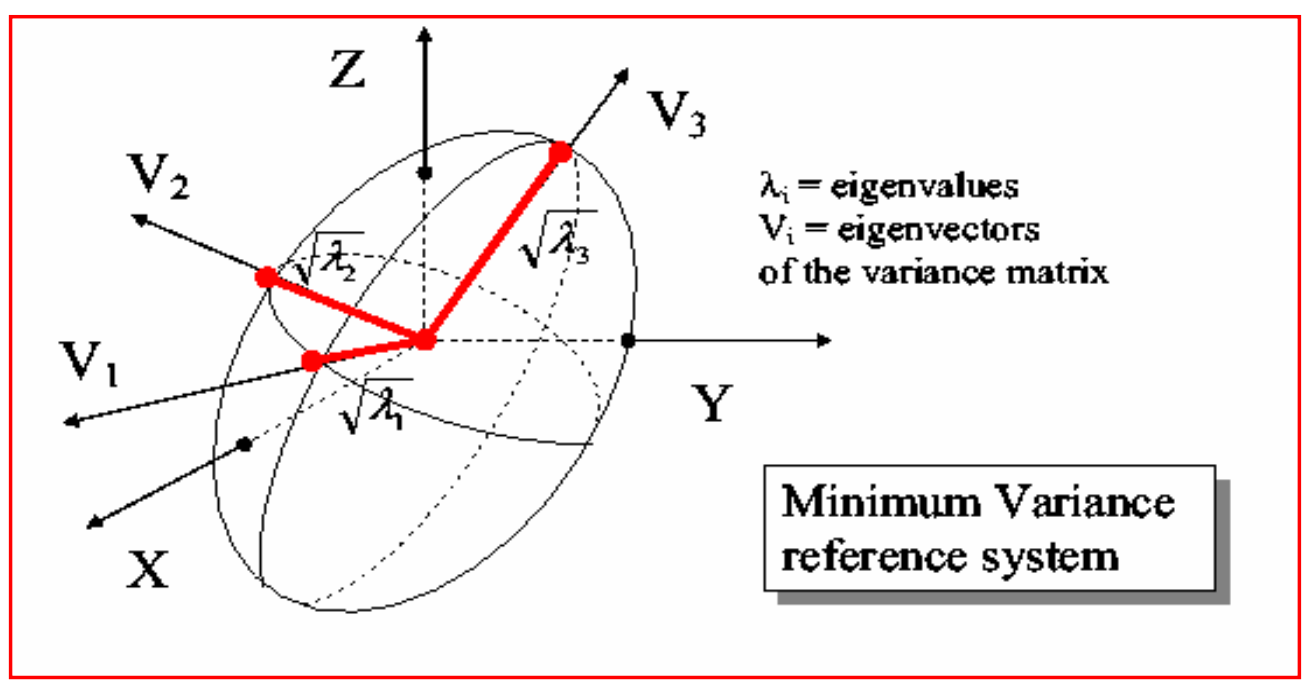

Figure 102: Original reference system $[x, y, z]$ and minimum variance reference system whose axes are $V_{1}, V_{2}$, and $V_{3}$ and represent the eigenvectors of $M$. Moreover, $\lambda_{1}, \lambda_{2}$, and $\lambda_{3}$ are the eigenvalues of $M$.

\subsection{The mean field reference system}

The mean field reference system (see Figure 103) reduces the problem of cross-talking between the components, due to the fact that the interplanetary magnetic field is not oriented like the axes of the reference system in which we perform the measurement. As a consequence, any component will experience a contribution from the other ones.

Let us suppose to have magnetic field data sampled in the RTN reference system. If the largescale mean magnetic field is oriented in the $[x, y, z]$ direction, we will look for a new reference system within the RTN reference system with the $x$ axis oriented along the mean field and the other two axes lying on a plane perpendicular to this direction.

Thus, we firstly determine the direction of the unit vector parallel to the mean field, normalizing its components

$$
\begin{aligned}
& e_{x 1}=B_{x} /|\mathbf{B}|, \\
& e_{x 2}=B_{y} /|\mathbf{B}|, \\
& e_{x 3}=B_{z} /|\mathbf{B}|,
\end{aligned}
$$

so that $\hat{e}_{x}^{\prime}\left(e_{x 1}, e_{x 2}, e_{x 3}\right)$ is the orientation of the first axis, parallel to the ambient field. As second direction it is convenient to choose the radial direction in RTN, which is roughly the direction of the solar wind flow, $\hat{e}_{R}(1,0,0)$. At this point, we compute a new direction perpendicular to the plane $\hat{e}_{R}-\hat{e}_{x}$

$$
\hat{e}_{z}^{\prime}\left(e_{z 1}, e_{z 2}, e_{z 3}\right)=\hat{e}_{x}^{\prime} \times \hat{e}_{R}
$$

Consequently, the third direction will be

$$
\hat{e}_{y}^{\prime}\left(e_{y 1}, e_{y 2}, e_{y 3}\right)=\hat{e}_{z}^{\prime} \times \hat{e}_{x}^{\prime}
$$

At this point, we can rotate our data into the new reference system. Data indicated as $B(x, y, z)$ in the old reference system, will become $B^{\prime}\left(x^{\prime}, y^{\prime}, z^{\prime}\right)$ in the new reference system. The transfor- 
mation is obtained applying the rotation matrix $A$

$$
A=\left(\begin{array}{ccc}
e_{x 1} & e_{x 2} & e_{x 3} \\
e_{y 1} & e_{y 2} & e_{y 3} \\
e_{z 1} & e_{z 2} & e_{z 3}
\end{array}\right)
$$

to the vector $B$, i.e., $B^{\prime}=A B$.

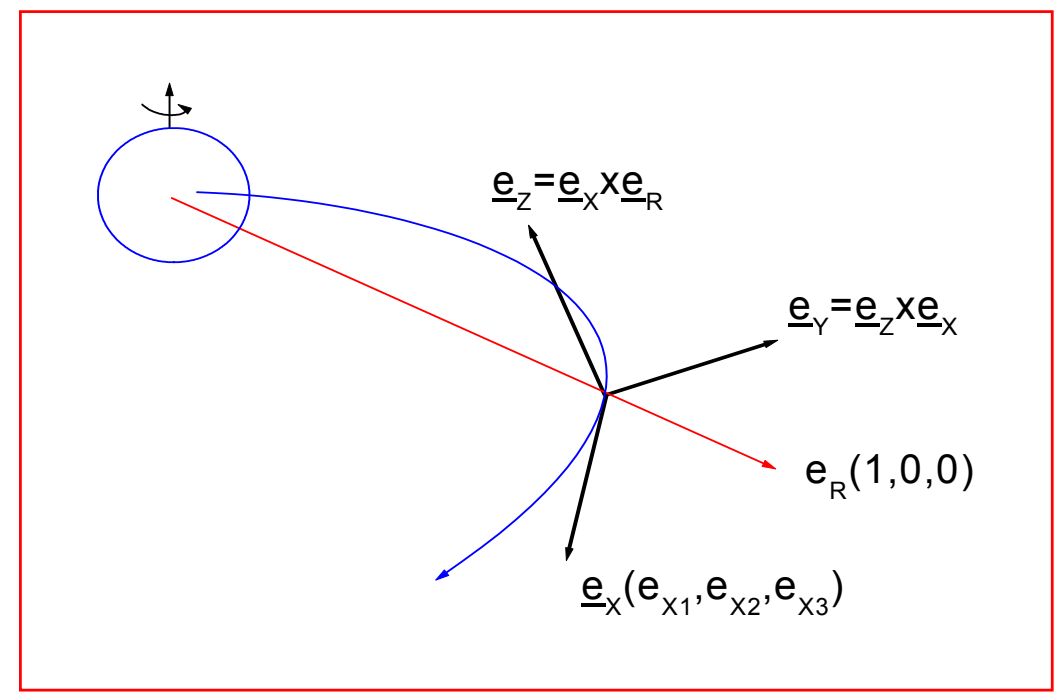

Figure 103: Mean field reference system. 


\section{Appendix E: On-board Plasma and Magnetic Field In- strumentation}

In this section, we briefly describe the working principle of two popular instruments commonly used on board spacecraft to measure magnetic field and plasma parameters. For sake of brevity, we will only concentrate on one kind of plasma and field instruments, i.e., the top-hat ion analyzer and the flux-gate magnetometer. Ample review on space instrumentation of this kind can be found, for example, in Pfaff et al. (1998a,b).

\subsection{Plasma instrument: The top-hat}

The top-hat electrostatic analyzer is a well known type of ion deflector and has been introduced by Carlson et al. (1982). It can be schematically represented by two concentric hemispheres, set to opposite voltages, with the outer one having a circular aperture centered around the symmetry axis (see Figure 104). This entrance allows charged particles to penetrate the analyzer for being detected at the base of the electrostatic plates by the anodes, which are connected to an electronic chain. To amplify the signal, between the base of the plates and the anodes are located the MicroChannelPlates (not shown in this picture). The MCP is made of a huge amount of tiny tubes, one close to the next one, able to amplify by a factor up to $10^{6}$ the electric charge of the incoming particle. The electron avalanche that follows hits the underlying anode connected to the electronic chain. The anode is divided in a certain number of angular sectors depending on the desired angular resolution.

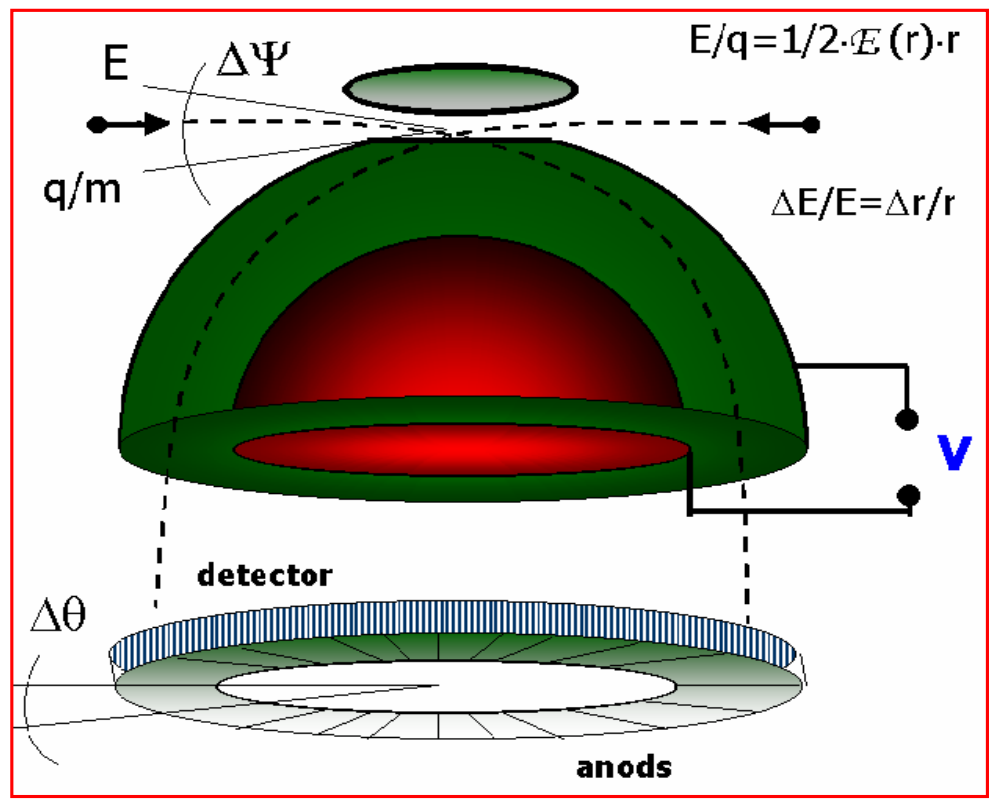

Figure 104: Outline of a top-hat plasma analyzer.

The electric field $E(r)$ generated between the two plates when an electric potential difference $\delta V$ is applied to them, is simply obtained applying the Gauss theorem and integrating between 
the internal $\left(R_{1}\right)$ and external $\left(R_{2}\right)$ radii of the analyzer

$$
E(r)=\delta V \frac{R_{1} R_{2}}{R_{1}-R_{2}} \frac{1}{r^{2}}
$$

In order to have the particle $q$ to complete the whole trajectory between the two plates and hit the detector located at the bottom of the analyzer, its centripetal force must be equal to the electric force acting on the charge. From this simple consideration we easily obtain the following relation between the kinetic energy of the particle $E_{k}$ and the electric field $E(r)$ :

$$
\frac{E_{k}}{q}=\frac{1}{2} E(r) r
$$

Replacing $E(r)$ with its expression from Equation (97) and differentiating, we get the energy resolution of the analyzer

$$
\frac{\delta E_{k}}{E_{k}}=\frac{\delta r}{r}=\text { const. }
$$

where $\delta r$ is the distance between the two plates. Thus, $\delta E_{k} / E_{k}$ depends only on the geometry of the analyzer. However, the field of view of this type of instrument is limited essentially to two dimensions since $\delta \Psi$ is usually rather small $\left(\sim 5^{\circ}\right)$. However, on a spinning s/c, a full coverage of the entire solid angle $4 \pi$ is obtained by mounting the deflector on the s/c, keeping its symmetry axis perpendicular to the s/c spin axis. In such a way the entire solid angle is covered during half period of spin.

Such an energy filter would be able to discriminate particles within a narrow energy interval $\left(E_{k}, E_{k}+\delta E_{k}\right)$ and coming from a small element $d \Omega$ of the solid angle. Given a certain energy resolution, the $3 \mathrm{D}$ particle velocity distribution function would be built sampling the whole solid angle $4 \pi$, within the energy interval to be studied.

\subsection{Measuring the velocity distribution function}

In this section, we will show how to reconstruct the average density of the distribution function starting from the particles detected by the analyzer. Let us consider the flux through a unitary surface of particles coming from a given direction. If $f\left(v_{x}, v_{y}, v_{z}\right)$ is the particle distribution function in phase space, $f\left(v_{x}, v_{y}, v_{z}\right) d v_{x} d v_{y} d v_{z}$ is the number of particles per unit volume $\left(\mathrm{pp} / \mathrm{cm}^{3}\right)$ with velocity between $v_{x}$ and $v_{x}+d v_{x}, v_{y}$ and $v_{y}+d v_{y}, v_{z}$ and $v_{z}+d v_{z}$, the consequent incident flux $\Phi_{i}$ through the unit surface is

$$
\Phi_{i}=\iiint v f d^{3} \omega
$$

where $d^{3} \omega=v^{2} d v \sin \theta d \theta d \phi$ is the unit volume in phase space (see Figure 105).

The transmitted flux $C^{t}$ will be less than the incident flux $\Phi_{i}$ because not all the incident particles will be transmitted and $\Phi_{i}$ will be multiplied by the effective surface $S(<1)$, i.e.,

$$
C^{t}=\iiint S v f d^{3} \omega=\iiint S v f v^{2} d v \sin \theta d \theta d \phi
$$

Since for a top-hat Equation 99 is valid, then

$$
v^{2} d v=v^{3} \frac{d v}{v} \sim v^{3}
$$

We have that the counts recorded within the unit phase space volume would be given by

$$
C_{\phi, \theta, v}^{t}=f_{\phi, \theta, v} S v^{4} \delta \theta \delta \phi \frac{d v}{v} \sin \theta=f_{\phi, \theta, v} v^{4} G
$$

Living Reviews in Solar Physics

http: //www . livingreviews . org/lrsp-2005-4 


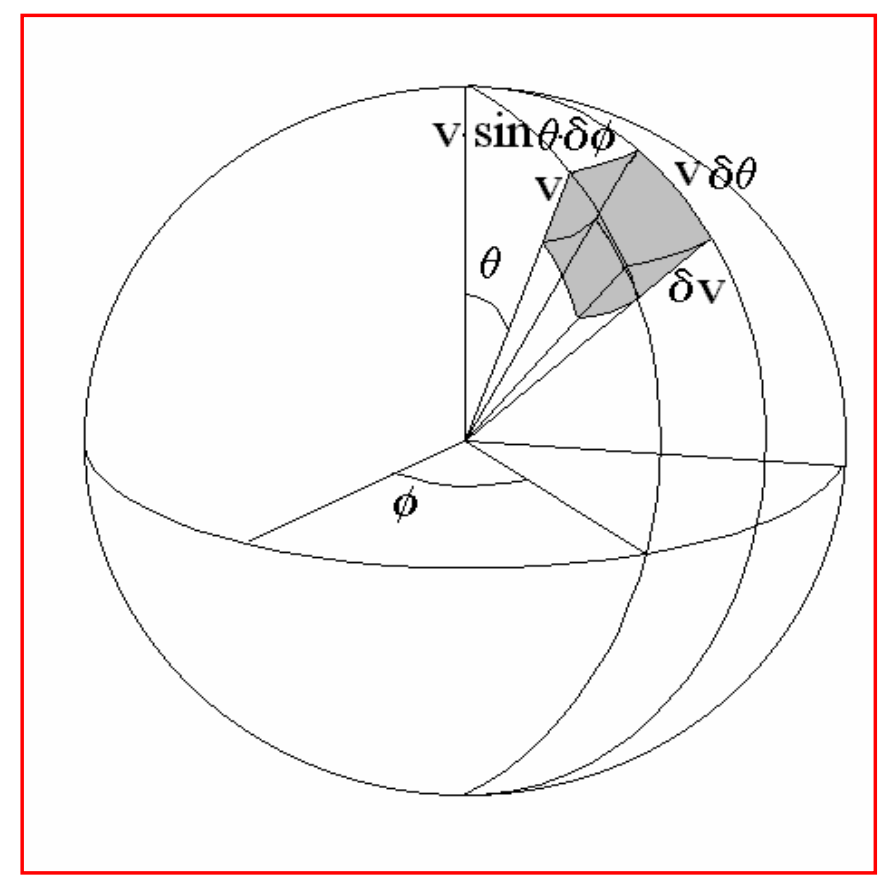

Figure 105: Unit volume in phase space.

where $G$ is called Geometrical Factor and is a characteristic of the instrument. Then, from the previous expression it follows that the phase space density function $f_{\phi, \theta, v}$ can be directly reconstructed from the counts

$$
f_{\phi, \theta, v}=\frac{C^{t}{ }_{\phi, \theta, v}}{v^{4} G} .
$$

\subsection{Computing the moments of the velocity distribution function}

Once we are able to measure the density particle distribution function $f_{\phi, \theta, v}$, we can compute the most used moments of the distribution in order to obtain the particle number density, velocity, pressure, temperature, and heat-flux (Paschmann et al., 1998).

If we simply indicate with $f(v)$ the density particle distribution function, we define as moment of order $n$ of the distribution the quantity $M_{n}$, i.e.,

$$
M_{n}=\int v_{n} f(v) d^{3} \omega
$$

It follows that the first 4 moments of the distribution are the following:

- the number density

$$
n=\int f(v) d^{3} \omega
$$

- the number flux density vector

$$
n \mathbf{V}=\int f(v) \mathbf{v} d^{3} \omega
$$


- the momentum flux density tensor

$$
\Pi=m \int f(v) \mathbf{v} \mathbf{v} d^{3} \omega,
$$

- the energy flux density vector

$$
\mathbf{Q}=\frac{m}{2} \int f(\mathbf{v}) v^{2} \mathbf{v} d^{3} \omega
$$

Once we have computed the zero-order moment, we can obtain the velocity vector from Equation (106). Moreover, we can compute $\Pi$ and $\mathbf{Q}$ in terms of velocity differences with respect to the bulk velocity, and Equations $(107,108)$ become

$$
\mathbf{P}=m \int f(\mathbf{v})(\mathbf{v}-\mathbf{V})(\mathbf{v}-\mathbf{V}) d^{3} \omega
$$

and

$$
\mathbf{H}=\frac{m}{2} \int f(\mathbf{v})|\mathbf{v}-\mathbf{V}|^{2}(\mathbf{v}-\mathbf{V}) d^{3} \omega .
$$

The new Equations $(109,110)$ represent the pressure tensor and the heat flux vector, respectively. Moreover, using the relation $\mathbf{P}=n K \mathbf{T}$ we extract the temperature tensor from Equations $(109,105)$. Finally, the scalar pressure $P$ and temperature $T$ can be obtained from the trace of the relative tensors

$$
P=\frac{\operatorname{Tr}\left(\mathbf{P}_{i j}\right)}{3}
$$

and

$$
T=\frac{\operatorname{Tr}\left(\mathbf{T}_{i j}\right)}{3} .
$$

\subsection{Field instrument: The flux-gate magnetometer}

There are two classes of instruments to measure the ambient magnetic field: scalar and vector magnetometers. While nuclear precession and optical pumping magnetometers are the most common scalar magnetometers used on board s/c (see Pfaff et al., 1998b for related material), the flux-gate magnetometer is, with no doubt, the mostly used one to perform vector measurements of the ambient magnetic field. In this section, we will briefly describe only this last instrument just for those who are not familiar at all with this kind of measurements in space.

The working principle of this magnetometer is based on the phenomenon of magnetic hysteresis. The primary element (see Figure 106) is made of two bars of high magnetic permeability material. A magnetizing coil is spooled around the two bars in an opposite sense so that the magnetic field created along the two bars will have opposite polarities but the same intensity. A secondary coil wound around both bars will detect an induced electric potential only in the presence of an external magnetic field.

The field amplitude $B B$ produced by the magnetizing field $H$ is such that the material periodically saturates during its hysteresis cycle as shown in Figure 107.

In absence of an external magnetic field, the magnetic field $B_{1}$ and $B_{2}$ produced in the two bars will be exactly the same but out of phase by $180^{\circ}$ since the two coils are spooled in an opposite sense. As a consequence, the resulting total magnetic field would be 0 as shown in Figure 107. In these conditions no electric potential would be induced on the secondary coil because the magnetic flux $\Phi$ through the secondary is zero.

On the contrary, in case of an ambient field $H_{\mathrm{A}} \neq 0$, its component parallel to the axis of the bar is such to break the symmetry of the resulting $B$ (see Figure 108). $H_{\mathrm{A}}$ represents an offset

Living Reviews in Solar Physics

http: //www . livingreviews . org/lrsp-2005-4 


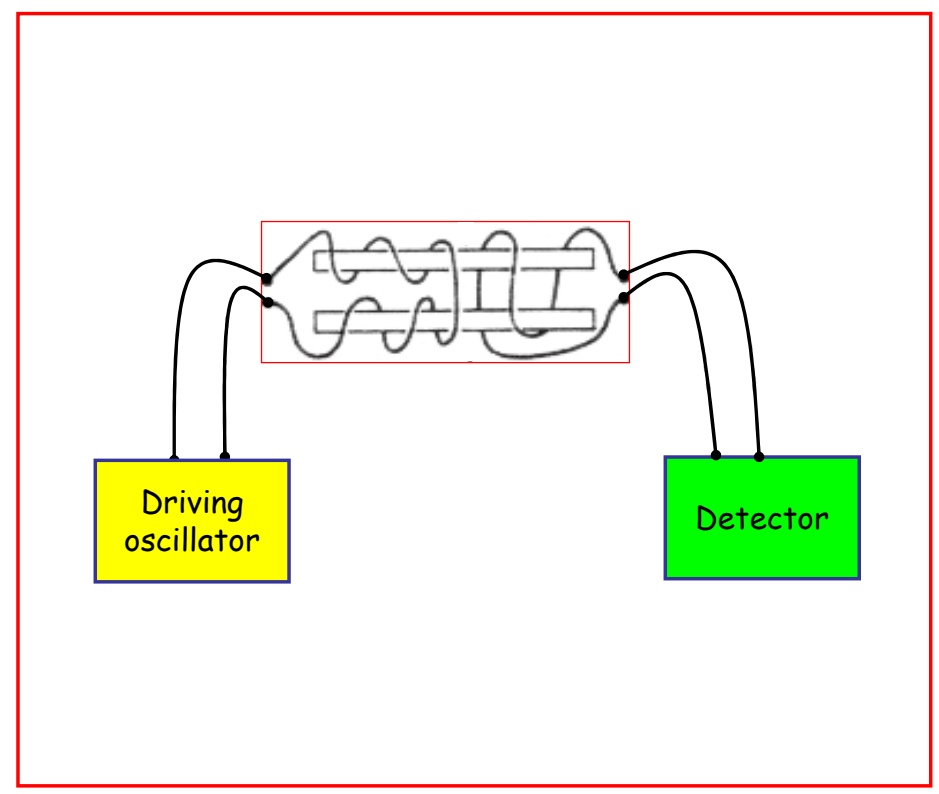

Figure 106: Outline of a flux-gate magnetometer. The driving oscillator makes an electric current, at frequency $f$, circulate along the coil. This coil is such to induce along the two bars a magnetic field with the same intensity but opposite direction so that the resulting magnetic field is zero. The presence of an external magnetic field breaks this symmetry and the resulting field $\neq 0$ will induce an electric potential in the secondary coil, proportional to the intensity of the component of the ambient field along the two bars. 


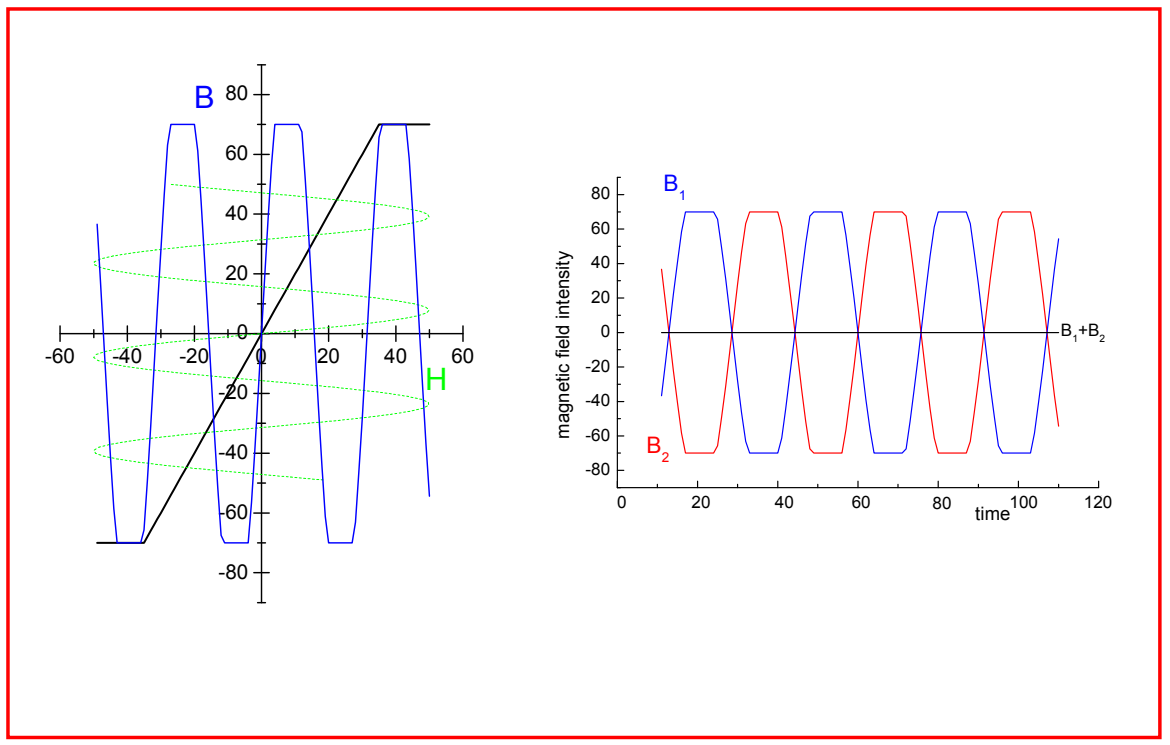

Figure 107: Left panel: This figure refers to any of the two sensitive elements of the magnetometer. The thick black line indicates the magnetic hysteresis curve, the dotted green line indicates the magnetizing field $H$, and the thin blue line represents the magnetic field $B$ produced by $H$ in each bar. The thin blue line periodically reaches saturation producing a saturated magnetic field $B$. The trace of $B$ results to be symmetric around the zero line. Right panel: magnetic fields $B_{1}$ and $B_{2}$ produced in the two bars, as a function of time. Since $B_{1}$ and $B_{2}$ have the same amplitude but out of phase by $180^{\circ}$, they cancel each other. 
that would add up to the magnetizing field $H$, so that the resulting field $B$ would not saturate in a symmetric way with respect to the zero line. Obviously, the other sensitive element would experience a specular effect and the resulting field $B=B_{1}+B_{2}$ would not be zero, as shown in Figure 108.

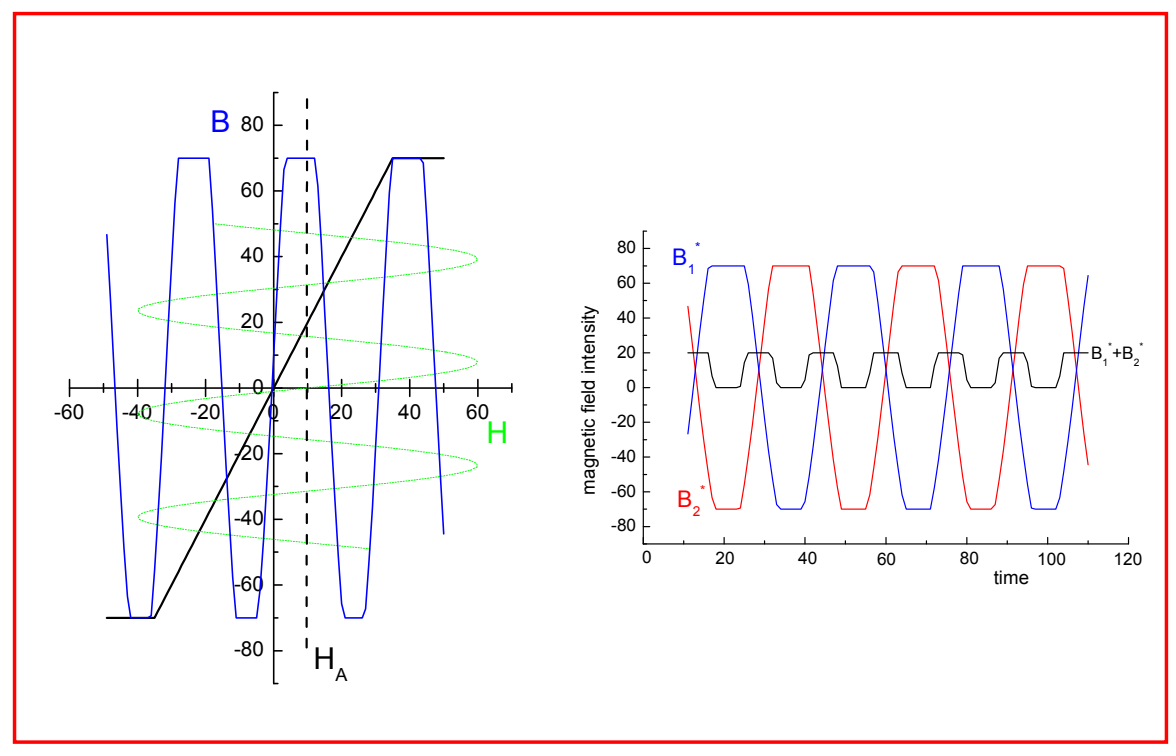

Figure 108: Left panel: the net effect of an ambient field $H_{\mathrm{A}}$ is that of introducing an offset which will break the symmetry of $B$ with respect to the zero line. This figure has to be compared with Figure 107 when no ambient field is present. The upper side of the $B$ curve saturates more than the lower side. An opposite situation would be shown by the second element. Right panel: trace of the resulting magnetic field $B=B_{1}+B_{2}$. The asymmetry introduced by $H_{\mathrm{A}}$ is such that the resulting field $B$ is different from zero.

In these conditions the resulting field $B$, fluctuating at frequency $f$, would induce an electric potential $V=-d \Phi / d t$, where $\Phi$ is the magnetic flux of $B$ through the secondary coil.

At this point, the detector would measure this voltage which would result proportional to the component of the ambient field $H_{\mathrm{A}}$ along the axis of the two bars. To have a complete measurement of the vector magnetic field $\mathbf{B}$ it will be sufficient to mount three elements on board the spacecraft, like the one shown in Figure 106, mutually orthogonal, in order to measure all the three Cartesian components. 


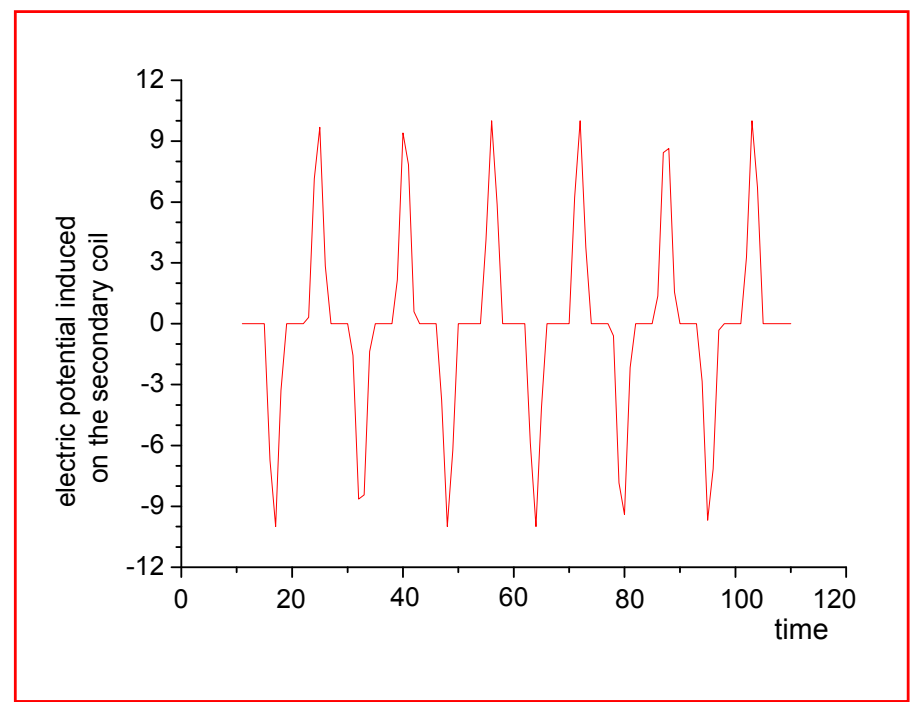

Figure 109: Time derivative of the curve $B=B_{1}+B_{2}$ shown in Figure 108 assuming the magnetic flux is referred to a unitary surface. 


\section{Appendix F: Spacecraft and Datasets}

Measurements performed by spacecrafts represent a unique chance to investigate a wide range of scales of low-frequency turbulence in a magnetized medium. The interested readers are strongly encouraged to visit the web pages of each specific space mission or, more simply, the "Virtual Space Physics Observatory" (http://vspo.gsfc.nasa.gov) as a wide source of information. In particular, the VSPO represents an easy way to access all the available datasets, related to magnetospheric and heliospheric missions, easily retrievable via "Space Physics Product Site Finder", a website that allows the user to quickly find data files and interfaces to data from a large number of space missions and ground based observations.

Two of the s/c which have contributed most to the study of MHD turbulence are the old HELIOS and VOYAGER spacecraft, which explored the inner and outer heliosphere, respectively, providing us with an almost complete map of the gross features of low-frequency plasma turbulence. The Helios project was a German-American mission consisting in two interplanetary probes: Helios 1, which was launched in December 1974, and Helios 2, launched one year later. These s/c had a highly elliptic orbit, lying in the ecliptic, which brought the s/c from $1 \mathrm{AU}$ to $0.3 \mathrm{AU}$ in only 6 months. Helios dataset is, with no doubt, the most important and unique one to study MHD turbulence in the inner heliosphere. Most of the knowledge we have today about this topic is based on Helios data mainly because this s/c is the only one that has gone so close to the Sun. As a matter of fact, the orbit of this s/c allowed to observe the radial evolution of turbulence within regions of space $(<0.7 \mathrm{AU})$ where dynamical processes between fast and slow streams have not yet reprocessed the plasma.

The two Voyagers were launched in 1977. One of them, Voyager 1 will soon reach the termination shock and enter the interstellar medium. As a consequence, for the first time, we will be able to measure interstellar particles and fields not affected by the solar wind. Within the study of MHD turbulence, the importance of the two Voyagers in the outer heliosphere is equivalent to that of the two Helios in the inner regions of the heliosphere. However, all these s/c have been limited to orbit in the, or close to the ecliptic plane.

Finally, in October 1990, Ulysses was launched and, after a fly-by with Jupiter it reached its final orbit tilted at $80.2^{\circ}$ with respect to the solar equator. For the first time, we were able to sample the solar wind coming from polar coronal holes, the pure fast wind not "polluted" by the dynamical interaction with slow equatorial wind. As a matter of fact, the Ulysses scientific mission has been dedicated to investigate the heliospheric environment out of the ecliptic plane. This mission is still providing exciting results.

Another spacecraft called WIND was launched in November 1994 and is part of the ISTP Project. WIND, was initially located at the Earth-Sun Lagrangian point $L 1$ to sample continuously the solar wind. Afterwards, it was moved to a much more complicated orbit which allows the spacecraft to repeatedly visit different regions of space around Earth, while continuing to sample the solar wind. The high resolution of magnetic field and plasma measurements of WIND makes this spacecraft very useful to investigate small scales phenomena, where kinetic effects start to play a key role.

The Advanced Composition Explorer (ACE) represents another solar wind monitor located at L1. This spacecraft was launched by NASA in 1997 and its solar wind instruments are characterized by a high rate sampling. Finally, we like to call the attention of the reader on the possibility to easily view and retrieve from the web real time solar wind data from both WIND and ACE.

Not far from now, when Voyager 2 will reach the termination shock we will have explored almost the whole heliosphere. However, the exploration will not be complete until we will reach the base of the solar corona. All the fundamental physical processes concurring during the birth of the solar wind take place in this part of the heliosphere. Moreover, this is a key region also for the study of turbulence, since here non-linear interactions between inward and outward modes start 
to be active and produce the turbulence spectrum that we observe in the heliosphere.

This region is so important for our understanding of the solar wind that both ESA and NASA are planning space mission dedicated to explore it. In particular, the European Space Agency is planning to launch the Solar Orbiter mission in October 2013 (http://www.esa.int/esaSC). Solar Orbiter is proposed as a space mission dedicated to study the solar surface, the corona, and the solar wind by means of remote sensing and in-situ measurements, respectively. Consequently, the s/c will carry a heliospheric package primarily designed to measure ions and electrons of the solar wind, energetic particles, radio waves, and magnetic fields and a remote sensing package for for EUV/X-ray spectroscopy and imaging of the disk and the extended corona. In particular, the high resolution imaging of the Sun will give close-up observations of the solar atmosphere, with the capability of resolving fine structures (below $100 \mathrm{~km}$ ) in the transition region and corona. This will certainly represent a major step forward in investigating the occurrence of intermittent structures of turbulence at very small scales.

The observations provided by Helios $25 \mathrm{yr}$ ago and, more recently, by Ulysses suggest that the local production of Alfvén waves is much stronger in the region just inside 0.3 AU, and Solar Orbiter, reaching 0.2 AU, will provide excellent observations to study problems related to local generation and non-linear coupling between outward and inward waves. Moreover, the high data sampling will provide extremely useful and totally new insight about wave dissipation via waveparticle coupling mechanism and the role that the damping of slow, fast, and Alfvén waves can have in the heating of the solar wind ions. Finally, the opportunity given by Solar Orbiter to correlate in-situ plasma measures with the simultaneous imaging of the same flow element of the solar wind during the co-rotation phase, will provide the possibility to separate temporal effects from spatial effects for the first time in the solar wind. This will be of primary importance for finally understand the physical mechanisms at the basis of the solar wind generation.

A similar mission, Solar Probe (http://solarprobe.gsfc.nasa.gov), is under development by NASA. The launch baseline is August 2012, and two solar polar passes at approximately 4 solar radii, within the sonic point of the wind, at different times in solar cycle, are foreseen. This mission, although very risky, will allow us to tremendously advance our knowledge about the physical processes that heat and accelerate the solar wind. Unfortunately, this mission has been facing serious funding problems and has a rather uncertain future.

Thus, future key missions for investigating turbulence properties in the solar wind plasma are not just behind the corner and, for the time being, we have to use observations from already flown or still flying spacecraft. This does not mean that exciting results are over, while we wait for these new missions. The main difference with the past is that now we are in a different phase of our research. This phase aims to refine many of the concepts we learned in the past, especially those concerning the radial evolution and the local production of turbulence. As a consequence more refined data analysis and computer simulations are now discovering new and very interesting aspects of MHD turbulence which, we hope, we contributed to illustrate in this review.

Living Reviews in Solar Physics

http: //www . livingreviews . org/lrsp-2005-4 


\section{References}

Balogh, A., Horbury, T.S., Forsyth, R.J., Smith, E.J., 1995, "Variances of the components and magnitude of the polar heliospheric magnetic field", in Solar Wind Eight, (Eds.) Winterhalter, D., Gosling, J.T., Habbal, S.R., Kurth, W.S., Neugebauer, M., Proceedings of the Eighth International Solar Wind Conference, Dana Point, CA 1995, vol. 382 of AIP Conference Proceedings, pp. 38-43, American Institute of Physics, Woodbury, U.S.A. Related online version (cited on 07 September 2005):

http://adsabs.harvard.edu/cgi-bin/bib_query?1995sowi.confQ. .38B. 4.1, 4.1

Balogh, A., Forsyth, R.J., Lucek, E.A., Horbury, T.S., Smith, E.J., 1999, "Heliospheric magnetic field polarity inversions at high heliographic latitudes", Geophys. Res. Lett., 26, 631-634. Related online version (cited on 07 September 2005):

http://adsabs.harvard.edu/cgi-bin/bib_query?1999GeoRL . .26 . 631B. 3.1

Barnes, A., 1979, "Hydromagnetic waves and turbulence in the solar wind", in Solar System Plasma Physics, (Eds.) Parker, E.N., Kennel, C.F., Lanzerotti, L.J., vol. 1, pp. 249-319, North-Holland, Amsterdam, Netherlands; New York, U.S.A. 6.1

Barnes, A., 1981, "Interplanetary Alfvénic fluctuations: a stochastic model", J. Geophys. Res., 86, 7498-7506. Related online version (cited on 07 September 2005):

http://adsabs.harvard.edu/cgi-bin/bib_query?1981JGR . . . 86.7498B. $\quad 3.2 .2$

Barnes, A., Hollweg, J.V., 1974, "Large-amplitude hydromagnetic waves", J. Geophys. Res., 79, 2302-2318. 3.1 .1

Batchelor, G.K., 1970, Theory of Homogeneous Turbulence, Cambridge University Press, Cambridge, U.K.; New York, U.S.A. Originally published 1953. 13, 13.2, 13.2

Bavassano, B., Bruno, R., 1989, "Evidence of local generation of Alfvénic turbulence in the solar wind", J. Geophys. Res., 94, 11 977-11 982. Related online version (cited on 07 September 2005): http://adsabs.harvard.edu/cgi-bin/bib_query?1989JGR . . .9411977B. 5.1, 9

Bavassano, B., Bruno, R., 1992, "On the role of interplanetary sources in the evolution of lowfrequency Alfvénic turbulence in the solar wind", J. Geophys. Res., 97, 19 129-19 137. Related online version (cited on 07 September 2005):

http://adsabs.harvard.edu/cgi-bin/bib_query?1992JGR . . .9719129B. 3.2.2, 5.1

Bavassano, B., Bruno, R., 1995, "Density fluctuations and turbulent Mach number in the inner solar wind", J. Geophys. Res., 100, 9475-9480. Related online version (cited on 07 September 2005):

http://adsabs.harvard.edu/cgi-bin/bib_query?1995JGR . . 100.9475B. 6.1, 6.2

Bavassano, B., Bruno, R., 2000, "Velocity and magnetic field fluctuations in Alfvénic regions of the inner solar wind: Three-fluid observations", J. Geophys. Res., 105, 5113-5118. Related online version (cited on 07 September 2005):

http://adsabs.harvard.edu/cgi-bin/bib_query?2000JGR . . 105.5113B. 3.1.8, 34, 3.1.8

Bavassano, B., Dobrowolny, M., Fanfoni, G., Mariani, F., Ness, N.F., 1982a, "Statistical properties of MHD fluctuations associated with high-speed streams from Helios 2 observations", Solar Phys., 78, 373-384. Related online version (cited on 07 September 2005):

http://adsabs.harvard.edu/cgi-bin/bib_query?1982SoPh...78. .373B. 3.1.3, 3.1.3, 3.1.5, $4.1,6,9.1$ 
Bavassano, B., Dobrowolny, M., Mariani, F., Ness, N.F., 1982b, "Radial evolution of power spectra of interplanetary Alfvénic turbulence", J. Geophys. Res., 87, 3617-3622. Related online version (cited on 07 September 2005):

http://adsabs.harvard.edu/cgi-bin/bib_query?1982JGR . . .87.3617B. 7, 3.1.2, 3.1.2, $3.2 .1,4.1,6$

Bavassano, B., Bruno, R., Klein, L., 1995, "Density-Temperature correlation in solar wind MHD fluctuations: a test for nearly incompressible models", J. Geophys. Res., 100, 5871-5875. Related online version (cited on 07 September 2005):

http://adsabs.harvard.edu/cgi-bin/bib_query?1995JGR . .100.5871B. 6.1, 6.2

Bavassano, B., Bruno, R., Rosenbauer, H., 1996a, "Compressive fluctuations in the solar wind and their polytropic index", Ann. Geophys., 14, 510-517. Related online version (cited on 07 September 2005):

http://adsabs.harvard.edu/cgi-bin/bib_query?1996AnGeo..14..510B. 6.1, 6.2

Bavassano, B., Bruno, R., Rosenbauer, H., 1996b, "MHD compressive turbulence in the solar wind and the nearly incompressible approach", Astrophys. Space Sci., 243, 159-169. 6.2

Bavassano, B., Woo, R., Bruno, R., 1997, "Heliospheric plasma sheet and coronal streamers", Geophys. Res. Lett., 24, 1655-1658. Related online version (cited on 07 September 2005):

http: //adsabs.harvard.edu/cgi-bin/bib_query?1997GeoRL. .24.1655B. 3.1, 20

Bavassano, B., Pietropaolo, E., Bruno, R., 1998, "Cross-helicity and residual energy in solar wind turbulence. Radial evolution and latitudinal dependence in the region from 1 to 5 AU", $J$. Geophys. Res., 103, 6521-6530. Related online version (cited on 07 September 2005): http://adsabs.harvard.edu/cgi-bin/bib_query?1998JGR...103.6521B. 4.2, 56

Bavassano, B., Pietropaolo, E., Bruno, R., 2000a, "On the evolution of outward and inward Alfvénic fluctuations in the polar wind", J. Geophys. Res., 105, 15959-15964. Related online version (cited on 07 September 2005):

http://adsabs.harvard.edu/cgi-bin/bib_query?2000JGR ...10515959B. 4.1, 4.2, 55, 4.2, 57

Bavassano, B., Pietropaolo, E., Bruno, R., 2000b, "Alfvénic turbulence in the polar wind: A statistical study on cross helicity and residual energy variations", J. Geophys. Res., 105, 12 69712 704. Related online version (cited on 07 September 2005):

http://adsabs.harvard.edu/cgi-bin/bib_query?2000JGR . . 10512697B. 3.1.8, 55, 4.2

Bavassano, B., Pietropaolo, E., Bruno, R., 2001, "Radial evolution of outward and inward Alfvénic fluctuations in the solar wind: A comparison between equatorial and polar observations by Ulysses", J. Geophys. Res., 106, $10659-10668$. Related online version (cited on 07 September 2005):

http://adsabs.harvard.edu/cgi-bin/bib_query?2001JGR . .10610659B. 39, 58

Bavassano, B., Pietropaolo, E., Bruno, R., 2002a, "Alfvénic turbulence in high-latitude solar wind: Radial versus latitudinal variations", J. Geophys. Res., 107, 1452. Related online version (cited on 07 September 2005):

http://adsabs.harvard.edu/cgi-bin/bib_query?2002JGRA.1071.SSH7B. 4.2, 57, 4.2

Bavassano, B., Pietropaolo, E., Bruno, R., 2002b, "On parametric instability and MHD turbulence evolution in high-latitude heliosphere", in Solspa 2001, (Ed.) Sawaya-Lacoste, H., Proceedings of the Second Solar Cycle and Space Weather Euroconference, 24-29 September 2001, Vico Equense, Italy, vol. SP-477 of ESA Conference Proceedings, pp. 313-316, ESA Publications

Living Reviews in Solar Physics

http: //www . livingreviews . org/lrsp-2005-4 
Division, Noordwijk, Netherlands. Related online version (cited on 07 September 2005): http://adsabs.harvard.edu/cgi-bin/bib_query?2002scsw.conf...313B. 4.2

Bavassano, B., Pietropaolo, E., Bruno, R., 2004, "Compressive fluctuations in high-latitude solar wind", Ann. Geophys., 22, 689-696. Related online version (cited on 07 September 2005):

http://adsabs.harvard.edu/cgi-bin/bib_query?2004AnGeo..22..689B. 6.2, 67, 68,69, 70

Belcher, J.W., Davis Jr, L., 1971, "Large-amplitude Alfvén waves in the interplanetary medium", J. Geophys. Res., 76, 3534-3563. 3.1, 3.1.3, 3.1.5, 3.1.7, 3.1.7, 3.1.8, 3.1.8, 3.2.1

Belcher, J.W., Solodyna, C.V., 1975, "Alfvén waves and directional discontinuities in the interplanetary medium", J. Geophys. Res., 80, 181-186. Related online version (cited on 07 September 2005):

http://adsabs.harvard.edu/cgi-bin/bib_query?1975JGR ...80..181B. 3.1, 3.1.3, 3.1.5, $29,3.1 .7$

Bendat, J.S., Piersol, A.G., 1971, Random Data: Analysis and Measurement Procedures, WileyInterscience, New York, U.S.A. 13.2.1

Benzi, R., Paladin, G., Vulpiani, A., Parisi, G., 1984, "On the multifractal nature of fully developed turbulence and chaotic systems", J. Phys. A, 17, 3521-3531. Related online version (cited on 07 September 2005):

http://adsabs.harvard.edu/cgi-bin/bib_query?1984JPhA...17.3521B. 7.4

Benzi, R., Ciliberto, S., Tripiccione, R., Baudet, C., Massaioli, F., Succi, S., 1993, "Extended self-similarity in turbulent flows", Phys. Rev. E, 48, 29-35. 9

Bianchini, L., Pietropaolo, E., Bruno, R., 1999, "An improved method for local intermittency recognition", in Magnetic Fields and Solar Processes, (Ed.) Wilson, A., Proceedings of the 9th European Meeting on Solar Physics, 12-18 September 1999, Florence, Italy, vol. SP-448 of ESA Conference Proceedings, pp. 1141-1146, ESA Publications Division, Noordwijk, Netherlands. Related online version (cited on 07 September 2005):

http://adsabs.harvard.edu/cgi-bin/bib_query?1999mfsp.conf.1141B. 14

Bieber, J.W., Wanner, W., Matthaeus, W.H., 1996, "Dominant two-dimensional solar wind turbulence with implications for cosmic ray transport", J. Geophys. Res., 101, 2511-2522. Related online version (cited on 07 September 2005):

http://adsabs .harvard.edu/cgi-bin/bib_query?1996JGR...101.2511B. 3.1 .5

Biskamp, D., 1993, Nonlinear Magnetohydrodynamics, vol. 1 of Cambridge Monographs on Plasma Physics, Cambridge University Press, Cambridge, U.K.; New York, U.S.A. 1, 2, 2.9, 5, 7

Biskamp, D., 2003, Magnetohydrodynamic Turbulence, Cambridge University Press, Cambridge, U.K.; New York, U.S.A. 1, 2

Boffetta, G., Carbone, V., Giuliani, P., Veltri, P., Vulpiani, A., 1999, "Power Laws in Solar Flares: Self-Organized Criticality or Turbulence?", Phys. Rev. Lett., 83, 4662-4665. Related online version (cited on 07 September 2005):

http://adsabs.harvard.edu/cgi-bin/bib_query?1999PhRvL. .83.4662B. 7.6

Bohr, T., Jensen, M.H., Paladin, G., Vulpiani, A., 1998, Dynamical Systems Approach to Turbulence, vol. 8 of Cambridge Nonlinear Science Series, Cambridge University Press, Cambridge, U.K. $\quad 1,2.6$ 
Boyd, T.J.M., Sanderson, J.J., 2003, The Physics of Plasmas, Cambridge University Press, Cambridge, U.K.; New York, U.S.A. 2

Braginskii, S.I., 1965, "Transport processes in a plasma", vol. 1 of Reviews of Plasma Physics, p. 205, Consultants Bureau, New York, U.S.A. 2.2

Bruno, R., 1992, "Inner heliosphere observations of MHD turbulence in the solar wind - Challenges to theory", in Solar Wind Seven, (Eds.) Marsch, E., Schwenn, R., Proceedings of the 3rd COSPAR Colloquium held in Goslar, Germany, 16-20 September 1991, vol. 3 of COSPAR Colloquia Series, pp. 423-428, Pergamon Press, Oxford, U.K.; New York, U.S.A. iii, 4.1

Bruno, R., Bavassano, B., 1991, "Origin of low cross-helicity regions in the solar wind", J. Geophys. Res., 96, 7841-7851. Related online version (cited on 07 September 2005): http://adsabs.harvard.edu/cgi-bin/bib_query?1991JGR...96.7841B. 38, 4.1, 4.2, 6, 6, 9.1

Bruno, R., Bavassano, B., 1992, "Evolution of the Alfvénic correlation in the solar wind", Nuovo Cimento $C$, 15, 599-605. Related online version (cited on 07 September 2005): http://adsabs.harvard.edu/cgi-bin/bib_query?1992NCimC. .15..599B. 4.1

Bruno, R., Bavassano, B., 1993, "Cross-helicity depletions in the inner heliosphere, and magnetic field and velocity fluctuation decoupling", Planet. Space Sci., 41, 677-685. Related online version (cited on 07 September 2005):

http://adsabs.harvard.edu/cgi-bin/bib_query?1993P\&SS ..41.677B. 6, 6.3, 71

Bruno, R., Dobrowolny, M., 1986, "Spectral measurements of magnetic energy and magnetic helicity between 0.29 and 0.97 AU", Ann. Geophys., 4, 17-22. Related online version (cited on 07 September 2005):

http://adsabs.harvard.edu/cgi-bin/bib_query?1986AnGeo..4...17B. 3.1.2, 3.1.5, 3.1.6, 3.1 .6

Bruno, R., Bavassano, B., Villante, U., 1985, "Evidence for long period Alfvén waves in the inner solar system", J. Geophys. Res., 90, 4373-4377. Related online version (cited on 07 September 2005):

http://adsabs.harvard.edu/cgi-bin/bib_query?1985JGR ...90.4373B. 29, 3.1.7, 3.1.8, $3.1 .8,3.2 .2,3.2 .2,4.1,4.1,4.2,6.1$

Bruno, R., Bavassano, B., Rosenbauer, H., Mariani, F., 1989, "On the local generation of interplanetary Alfvénic fluctuations", Adv. Space Res., 9, 131-133. Related online version (cited on 07 September 2005):

http://adsabs.harvard.edu/cgi-bin/bib_query?1989AdSpR ...9. .131B. 3.2 .1

Bruno, R., Bavassano, B., Pietropaolo, E., 1996, "On the nature of Alfvénic inward modes in the solar wind", in Solar Wind Eight, (Eds.) Winterhalter, D., Gosling, J.T., Habbal, S.R., Kurth, W.S., Neugebauer, M., Proceedings of the Eighth International Solar Wind Conference, Dana Point, CA 1995, vol. 382 of AIP Conference Proceedings, pp. 229-232, American Institute of Physics, Woodbury, U.S.A. 41, 3.2.2, 42, 3.2.2, 5.1, 6

Bruno, R., Bavassano, B., Bianchini, L., Pietropaolo, E., Villante, U., Carbone, V., Veltri, P., 1999a, "Solar wind intermittency studied via Local Intermittency Measure", in Magnetic Fields and Solar Processes, (Ed.) Wilson, A., The 9th European Meeting on Solar Physics, held 12-18 September, 1999, in Florence, Italy, vol. SP-448 of ESA Conference Proceedings, pp. 1147-1152, ESA, Noordwijk, Netherlands. Related online version (cited on 07 September 2005): http://adsabs.harvard.edu/cgi-bin/bib_query?1999mfsp.conf.1147B. 14

Living Reviews in Solar Physics

http: //www . livingreviews . org/lrsp-2005-4 
Bruno, R., Bavassano, B., Pietropaolo, E., Carbone, V., Veltri, P., 1999b, "Effects of intermittency on interplanetary velocity and magnetic field fluctuations anisotropy", Geophys. Res. Lett., 26, 3185-3188. Related online version (cited on 07 September 2005):

http://adsabs.harvard.edu/cgi-bin/bib_query?1999GeoRL. .26.3185B. 3.1.3, 9.1

Bruno, R., Carbone, V., Veltri, P., Pietropaolo, E., Bavassano, B., 2001, "Identifying intermittent events in the solar wind", Planet. Space Sci., 49, 1201-1210. Related online version (cited on 07 September 2005):

http://adsabs.harvard.edu/cgi-bin/bib_query?2001P\&SS...49.1201B. 9, 87, 88, 89, 92, 9

Bruno, R., Carbone, V., Sorriso-Valvo, L., Bavassano, B., 2003a, "On the role of coherent and stochastic fluctuations in the evolving solar wind MHD turbulence: Intermittency", in Solar Wind Ten, (Eds.) Velli, M., Bruno, R., Malara, F., Proceedings of the Tenth International Solar Wind Conference, Pisa, Italy, 17-21 June 2002, vol. 679 of AIP Conference Proceedings, pp. 453-456, American Institute of Physics, Melville, U.S.A. 9.1, 9.1, 9.2

Bruno, R., Carbone, V., Sorriso-Valvo, L., Bavassano, B., 2003b, "Radial evolution of solar wind intermittency in the inner heliosphere", J. Geophys. Res., 108, 8-24. Related online version (cited on 07 September 2005):

http://adsabs .harvard.edu/cgi-bin/bib_query?2003JGRA.108c.SSH8B. 8.1, 9.1, 94, 95, 9.2

Burlaga, L.F., 1991a, "Intermittent turbulence in the solar wind", J. Geophys. Res., 96, 58475851. Related online version (cited on 07 September 2005):

http://adsabs.harvard.edu/cgi-bin/bib_query?1991JGR....96.5847B. 7,8.1

Burlaga, L.F., 1991b, "Multifractal structure of the interplanetary magnetic field - Voyager 2 observations near 25 AU, 1987-1988", Geophys. Res. Lett., 18, 69-72. Related online version (cited on 07 September 2005):

http://adsabs.harvard.edu/cgi-bin/bib_query?1991GeoRL. 18_.669. 7, 8.1

Burlaga, L.F., 1991c, "Multifractal structure of speed fluctuations in recurrent streams at 1 AU and near 6 AU", Geophys. Res. Lett., 18, 1651-1654. Related online version (cited on 07 September 2005):

http://adsabs.harvard.edu/cgi-bin/bib_query?1991GeoRL. .18.1651B. 7, 8.1

Burlaga, L.F., 1992a, "Multifractal structure of the magnetic field and plasma in recurrent streams at 1 AU", J. Geophys. Res., 97, 4283-4293. Related online version (cited on 07 September 2005): http://adsabs.harvard.edu/cgi-bin/bib_query?1992JGR....97.4283B. 4.1

Burlaga, L.F., 1992b, "Multifractals in the solar wind", in Solar Wind Seven, (Eds.) Marsch, E., Schwenn, R., Proceedings of the 3rd COSPAR Colloquium held in Goslar, Germany, 16-20 September 1991, vol. 3 of COSPAR Colloquia Series, pp. 429-432, Pergamon Press, Oxford, U.K.; New York, U.S.A. 4.1

Burlaga, L.F., 1993, "Intermittent turbulence in large-scale velocity fluctuations at 1 AU near solar maximum", J. Geophys. Res., 98, 17 467-17 474. Related online version (cited on 07 September 2005):

http://adsabs.harvard.edu/cgi-bin/bib_query?1993JGR ....9817467B. 1

Burlaga, L.F., 1995, Interplanetary magnetohydrodynamics, vol. 3 of International Series on Astronomy and Astrophysics, Oxford University Press, New York, U.S.A. 1, 7 
Burlaga, L.F., Klein, L.W., 1986, "Fractal structure of the interplanetary magnetic field", J. Geophys. Res., 91, 347-350. 4.1, 8.1

Burlaga, L.F., Ogilvie, K.W., 1970, "Magnetic and Thermal Pressures in the Solar Wind", Solar Phys., 15, 61-99. Related online version (cited on 07 September 2005):

http://adsabs.harvard.edu/cgi-bin/bib_query?1970SoPh...15..61B. 6.1

Burlaga, L.F., Turner, J.M., 1976, "Microscale 'Alfvén waves' in the solar wind at 1 AU", J. Geophys. Res., 81, 73-77. Related online version (cited on 07 September 2005):

http://adsabs.harvard.edu/cgi-bin/bib_query?1976JGR . . .81 . .73B. 3.1 .3

Carbone, V., 1993, "Cascade model for intermittency in fully developed magnetohydrodynamic turbulence", Phys. Rev. Lett., 71, 1546-1548. Related online version (cited on 07 September 2005):

http://adsabs.harvard.edu/cgi-bin/bib_query?1987A\&A . .188. .239C. 1, 2.9, 7, 7.4, 8.1

Carbone, V., 1994a, "Scale similarity of the velocity structure functions in fully developed magnetohydrodynamic turbulence", Phys. Rev. E, 50, 671-674. Related online version (cited on 07 September 2005):

http://adsabs.harvard.edu/cgi-bin/bib_query?1994PhRvE. .50..671C. 8.1

Carbone, V., 1994b, "Scaling exponents of the velocity structure functions in the interplanetary medium", Ann. Geophys., 12, 585-590. Related online version (cited on 07 September 2005): http://adsabs.harvard.edu/cgi-bin/bib_query?1994AnGeo..12..585C. 8.1

Carbone, V., Pouquet, A., 2005, "An introduction to fluid and MHD turbulence for astrophysical flows: Theory, observational and numerical data, and modeling", Springer, Berlin, Germany; New York, U.S.A. In press. 7

Carbone, V., Veltri, P., 1987, "A simplified cascade model for MHD turbulence", Astron. Astrophys., 188, 239-250. 5.1

Carbone, V., Veltri, P., 1990, "A shell model for anisotropic magnetohydrodynamic turbulence", Geophys. Astrophys. Fluid Dyn., 52, 153-181. 3.1.4, 3.1.4

Carbone, V., Veltri, P., 1992, "Relaxation processes in magnetohydrodynamics: a triad-interaction model", Astron. Astrophys., 259, 359-372. Related online version (cited on 07 September 2005): http://adsabs.harvard.edu/cgi-bin/bib_query?1992A\&A . . 259 . .359C. 5.1

Carbone, V., Malara, F., Veltri, P., 1995a, "A model for the three-dimensional magnetic field correlation spectra of low-frequency solar wind fluctuations during Alfvénic periods", J. Geophys. Res., 100, 1763-1778. Related online version (cited on 07 September 2005): http://adsabs .harvard.edu/cgi-bin/bib_query?1995JGR . . 100.1763C. 3.1.5, 3.1.5

Carbone, V., Veltri, P., Bruno, R., 1995b, "Experimental Evidence for Differences in the Extended Self-Similarity Scaling Laws between Fluid and Magnetohydrodynamic Turbulent Flows", Phys. Rev. Lett., 75, 3110-3113. Related online version (cited on 07 September 2005): http://adsabs.harvard.edu/cgi-bin/bib_query?1995PhRvL..75.3110C. 8.1, 9

Carbone, V., Bruno, R., Veltri, P., 1996a, "Evidences for extended self-similarity in hydromagnetic turbulence", Geophys. Res. Lett., 23, 121-124. Related online version (cited on 07 September 2005):

http://adsabs.harvard.edu/cgi-bin/bib_query?1996GeoRL . 23..121C. 9,8.1

Living Reviews in Solar Physics

http: //www . livingreviews . org/lrsp-2005-4 
Carbone, V., Veltri, P., Bruno, R., 1996b, "Solar wind low-frequency magnetohydrodynamic turbulence: extended self-similarity and scaling laws", Nonlinear Proc. Geophys., 3, 247-261. Related online version (cited on 07 September 2005):

http://adsabs.harvard.edu/cgi-bin/bib_query?1996NPGeo...3..247C. 7.4

Carbone, V., Sorriso-Valvo, L., Martines, E., Antoni, V., Veltri, P., 2000, "Intermittency and turbulence in a magnetically confined fusion plasma", Phys. Rev. E, 62, 49-56. Related online version (cited on 07 September 2005):

http://adsabs.harvard.edu/cgi-bin/bib_query?2000PhRvE..62 ..49C. 9, 76

Carlson, C.W., Curtis, D.W., Paschmann, G., Michael, W., 1982, "An instrument for rapidly measuring plasma distribution functions with high resolution", Adv. Space Res., 2, 67-70. Related online version (cited on 07 September 2005):

http://adsabs.harvard.edu/cgi-bin/bib_query?1982AdSpR...2R. .67C. 16.1

Castaing, B., Gagne, Y., Hopfinger, V., 2001, "Velocity probability density functions of high Reynolds number turbulence", Physica D, 46, 177-200. 9.2

Chandrasekhar, S., 1967, An introduction to the study of stellar structure, Dover, New York, U.S.A. 6.1

Chang, S.C., Nishida, A., 1973, "Spatial Structure of Transverse Oscillations in the Interplanetary Magnetic Field", Astrophys. Space Sci., 23, 301-301. 3.1 .3

Chang, T., 2003, "Complexity Induced Plasma Turbulence in Coronal Holes and the Solar Wind", in Solar Wind Ten, (Eds.) Velli, M., Bruno, R., Malara, F., Proceedings of the Tenth International Solar Wind Conference, Pisa, Italy, 17-21 June 2002, vol. 679 of AIP Conference Proceedings, pp. 481-484, American Institute of Physics, Melville, U.S.A. 9

Chang, T., Wu, C., 2002, "Complexity and anomalous transport in space plasmas", Phys. Plasmas, 9, 3679-3684. Related online version (cited on 07 September 2005):

http://adsabs.harvard.edu/cgi-bin/bib_query?2002PhPl...9.3679C. 9

Chang, T., Tam, S.W.Y., Wu, C., 2004, "Complexity induced anisotropic bimodal intermittent turbulence in space plasmas", Phys. Plasmas, 11, 1287-1299. Related online version (cited on 07 September 2005):

http://adsabs.harvard.edu/cgi-bin/bib_query?2004PhPl...11.1287C. 9, 93

Chian, A.C.L., Borotto, F.A., Gonzalez, W.D., 1998, "Alfvén Intermittent Turbulence Driven by Temporal Chaos", Astrophys. J., 505, 993-998. Related online version (cited on 07 September 2005):

http://adsabs.harvard.edu/cgi-bin/bib_query?1998ApJ...505..993C. 4

Chian, A.C.L., Rempel, E.L., Macau, E.E.N., Rosa, R.R., Christiansen, F., 2003, "Alfvén Turbulence Driven by High-Dimensional Interior Crisis in the Solar Wind", in Solar Wind Ten, (Eds.) Velli, M., Bruno, R., Malara, F., Proceedings of the Tenth International Solar Wind Conference, Pisa, Italy, 17-21 June 2002, vol. 679 of AIP Conference Proceedings, pp. 558-561, American Institute of Physics, Melville, U.S.A. 4

Cho, J.Y.N., Newell, R.E., Sachse, G.W., 2000, "Anomalous scaling of mesoscale tropospheric humidity fluctuations", Geophys. Res. Lett., 27, 377-380. Related online version (cited on 07 September 2005):

http://adsabs.harvard.edu/cgi-bin/bib_query?2000GeoRL..27 ..377C. 9.2 
Coleman, P.J., 1968, "Turbulence, Viscosity, and Dissipation in the Solar-Wind Plasma", Astrophys. J., 153, 371. Related online version (cited on 07 September 2005):

http://adsabs.harvard.edu/cgi-bin/bib_query?1968ApJ . . 153..371C. 3.1.1, 21

Courant, R., Friedrichs, K.O., 1976, Supersonic Flow and Shock Waves, vol. 21 of Applied Mathematical Sciences, Springer, Berlin, Germany; New York, U.S.A. 6.1

Dasso, S., Milano, L.J., Matthaeus, W.H., Smith, C.W., 2003, "Cross-helicity correlations in the solar wind", in Solar Wind Ten, (Eds.) Velli, M., Bruno, R., Malara, F., Proceedings of the Tenth International Solar Wind Conference, Pisa, Italy, 17-21 June 2002, vol. 679 of AIP Conference Proceedings, pp. 546-549, American Institute of Physics, Melville, U.S.A. 3.1.5

Del Zanna, L., 2001, "Parametric decay of oblique arc-polarized Alfvén waves", Geophys. Res. Lett., 28, 2585-2588. Related online version (cited on 07 September 2005): http: //adsabs.harvard.edu/cgi-bin/bib_query?2001GeoRL . .28.2585D. 5.1

Del Zanna, L., Velli, M., Londrillo, P., 2001, "Parametric decay of circularly polarized Alfvén waves: Multidimensional simulations in periodic and open domains", Astron. Astrophys., 367, 705-718. Related online version (cited on 07 September 2005): http://adsabs.harvard.edu/cgi-bin/bib_query?2001A\&A...367..705D. 5.1, 5.2

Denskat, K.U., Neubauer, F.M., 1983, "Observations of hydromagnetic turbulence in the solar wind", in Solar Wind Five, (Ed.) Neugebauer, M., Proceedings of a conference held in Woodstock, Vermont, November 1-5 1982, vol. 2280 of NASA Conference Publication, pp. 81-91, NASA, Washington, U.S.A. 3.1.2, 3.2.1

Derby, N.F., 1978, "Modulational instability of finite-amplitude, circularly polarized Alfvén waves", Astrophys. J., 224, 1013-1016. Related online version (cited on 07 September 2005): http://adsabs.harvard.edu/cgi-bin/bib_query?1978ApJ . . 224.1013D. 3.2 .2

Dobrowolny, M., Mangeney, A., Veltri, P., 1980a, "Fully developed anisotropic hydromagnetic turbulence in interplanetary space", Phys. Rev. Lett., 45, 144-147. Related online version (cited on 07 September 2005):

http://adsabs.harvard.edu/cgi-bin/bib_query?1980PhRvL . 45. .144D. 3.2

Dobrowolny, M., Mangeney, A., Veltri, P., 1980b, "Properties of magnetohydrodynamic turbulence in the solar wind", Astron. Astrophys., 83, 26-32. Related online version (cited on 07 September 2005):

http://adsabs.harvard.edu/cgi-bin/bib_query?1980A\&A ...83...26D. 2.9, 3.1.7, 3.2, $5.1,13.3$

Doob, J.L., 1953, Stochastic Processes, Wiley, New York, U.S.A. 13

Dudok de Wit, T., 2004, "Can high-order moments be meaningfully estimated from experimental turbulence measurements?", Phys. Rev. E, 70, $055302(\mathrm{R}) .9$

Elsässer, W.M., 1950, "The hydromagnetic equations", Phys. Rev., 79, $183 . \quad$ 2.2, 13.3

Farge, M., 1992, "Wavelet transforms and their applications to turbulence", Annu. Rev. Fluid Mech., 24, 395-457. Related online version (cited on 07 September 2005):

http://adsabs.harvard.edu/cgi-bin/bib_query?1992AnRFM. .24_.395F. 9, 9, 9.1, 14,14

Farge, M., Holschneider, M., Colonna, J.F., 1990, "Wavelet analysis of coherent structures in two-dimensional turbulent flows", in Topological Fluid Mechanics, (Ed.) Moffat, H.K., Proceedings of the IUTAM Symposium, Cambridge, UK, 13-18 August 1989, pp. 765-766, Cambridge University Press, Cambridge, U.K.; New York, U.S.A. 9.1, 14, 14

Living Reviews in Solar Physics

http: //www. livingreviews.org/lrsp-2005-4 
Feynman, R.P., Leighton, R.B., Sands, M., 1977, The Feynman Lectures on Physics, Vol. II: The Electromagnetic Field, Addison-Wesley, Reading, U.S.A., 6th edn. 4

Forman, M.A., Burlaga, L.F., 2003, "Exploring the Castaing Distribution Function to Study Intermittence in the Solar Wind at L1 in June 2000", in Solar Wind Ten, (Eds.) Velli, M., Bruno, R., Malara, F., Proceedings of the Tenth International Solar Wind Conference, Pisa, Italy, 17-21 June 2002, vol. 679 of AIP Conference Proceedings, pp. 554-557, American Institute of Physics, Melville, U.S.A. 8.2

Forsyth, B., Breen, A., 2002, "Meeting report: The 3-D Sun and heliosphere at solar maximum", Astron. Geophys., 43, 3.32. Related online version (cited on 07 September 2005): http://adsabs.harvard.edu/cgi-bin/bib_query?2002A\&G....43c..32F. 3.1

Forsyth, F.J., Balogh, A., Horbury, T.S., Smith, E.J., 1997, "The heliospheric magnetic field at solar minimum as observed by ULYSSES", Adv. Space Res., 19, 839-842. Related online version (cited on 07 September 2005):

http://adsabs.harvard.edu/cgi-bin/bib_query?1996A\&A...316..287F. 3.1

Forsyth, R.J., Horbury, T.S., Balogh, A., Smith, E.J., 1996, "Hourly variances of fluctuations in the heliospheric magnetic field out of the ecliptic plane", Geophys. Res. Lett., 23, 595-598. Related online version (cited on 07 September 2005):

http://adsabs.harvard.edu/cgi-bin/bib_query?1996GeoRL. .23..595F. 4.1, 45, 4.1, 4.1, $49,4.1,4.2$

Frick, P., Sokoloff, D.D., 1998, "Cascade and dynamo action in a shell model of magnetohydrodynamic turbulence", Phys. Rev. E, 57, 4155-4164. Related online version (cited on 07 September 2005):

http://adsabs.harvard.edu/cgi-bin/bib_query?1998PhRvE..57.4155F. 2.7

Frisch, U., 1995, Turbulence: The Legacy of A.N. Kolmogorov, Cambridge University Press, Cambridge, U.K.; New York, U.S.A. $1,1,3,4,2,2.3,3,6,6,2.10,7.1,9$, i, 7.3, 7.3, 9.1

Frisch, U., Pouquet, A., Leorat, J., Mazure, A., 1975, "Possibility of an inverse cascade of magnetic helicity in magnetohydrodynamic turbulence", J. Fluid Mech., 68, 769-778. Related online version (cited on 07 September 2005):

http://adsabs.harvard.edu/cgi-bin/bib_query?1975JFM....68..769F. 13.1

Galeev, A.A., Oraevskii, V.N., 1963, "The stability of Alfvén waves", Sov. Phys. Dokl., 7, 9881003. 3.2 .2

Ghosh, S., Matthaeus, W.H., 1990, "Relaxation processes in a turbulent compressible magnetofluid", Phys. Fluids B, 2, 1520-1534. Related online version (cited on 07 September 2005): http://adsabs.harvard.edu/cgi-bin/bib_query?1990PhFlB...2.1520G. 5.1

Ghosh, S., Papadopoulos, K., 1987, "The onset of Alfvénic turbulence", Phys. Fluids, 30, 13711387. Related online version (cited on 07 September 2005):

http://adsabs.harvard.edu/cgi-bin/bib_query?1987PhFl...30.1371G. 4

Ghosh, S., Matthaeus, W.H., Roberts, D.A., Goldstein, M.L., 1998a, "Waves, structures, and the appearance of two-component turbulence in the solar wind", J. Geophys. Res., 103, 2370523 716. Related online version (cited on 07 September 2005):

http://adsabs.harvard.edu/cgi-bin/bib_query?1998JGR...10323705G. 3.1 .5 
Ghosh, S., Matthaeus, W.H., Roberts, D.A., Goldstein, M.L., 1998b, "The evolution of slab fluctuations in the presence of pressure-balanced magnetic structures and velocity shears", J. Geophys. Res., 103, 23 691-23 704. Related online version (cited on 07 September 2005):

http://adsabs.harvard.edu/cgi-bin/bib_query?1998JGR . . 10323691G. 3.1 .5

Giuliani, P., Carbone, V., 1998, "A note on shell models for MHD turbulence", Europhys. Lett., 43, 527-532. Related online version (cited on 07 September 2005):

http://adsabs.harvard.edu/cgi-bin/bib_query?1998EL....43. .527G. 2.7, 2.7, 7.6, 7.6

Gledzer, E.B., 1973, "System of hydrodynamic type admitting two quadratic integrals of motion", Sov. Phys. Dokl., 18, 216. 2.7

Goldstein, B.E., Smith, E.J., Balogh, A., Horbury, T.S., Goldstein, M.L., Roberts, D.A., 1995a, "Properties of magnetohydrodynamic turbulence in the solar wind as observed by Ulysses at high heliographic latitudes", Geophys. Res. Lett., 22, 3393-3396. Related online version (cited on 07 September 2005):

http://adsabs.harvard.edu/cgi-bin/bib_query?1995GeoRL..22.3393G. 4.1, 4.1, 4.2, 53, 54

Goldstein, M.L., 1978, "An instability of finite amplitude circularly polarized Alfvén waves", Astrophys. J., 219, 700-704. Related online version (cited on 07 September 2005):

http://adsabs.harvard.edu/cgi-bin/bib_query?1978ApJ ...219..700G. 3.2.2, 5.2

Goldstein, M.L., Roberts, D.A., Matthaeus, W.H., 1986, "Systematic errors in determining the propagation direction of interplanetary Alfvénic fluctuations", J. Geophys. Res., 91, 1335713 365. Related online version (cited on 07 September 2005):

http://adsabs.harvard.edu/cgi-bin/bib_query?1986JGR. . .99113357G. 13.3

Goldstein, M.L., Roberts, D.A., Matthaeus, W.H., 1989, "Numerical Simulation of Interplanetary and Magnetospheric Phenomena: The Kelvin-Helmholtz Instability", in Solar System Plasma Physics, (Eds.) Waite Jr, J.H., Burch, J.L., Moore, R.L., Papers presented at the Yosemite Conference on Outstanding Problems in Solar System Plasma Physics: Theory and Instrumentation, Yosemite National Park, Calif., February 2-5, 1988, vol. 54 of Geophysical Monograph, p. 113, American Institute of Physics, Washington, U.S.A. 5.1

Goldstein, M.L., Roberts, D.A., Fitch, C.A., 1991, "The structure of helical interplanetary magnetic fields", Geophys. Res. Lett., 18, 1505-1508. Related online version (cited on 07 September 2005):

http://adsabs.harvard.edu/cgi-bin/bib_query?1991GeoRL. .18.1505G. 3.1.6

Goldstein, M.L., Roberts, D.A., Matthaeus, W.H., 1995b, "Magnetohydrodynamic Turbulence in the Solar Wind", Annu. Rev. Astron. Astrophys., 33, 283-326. Related online version (cited on 07 September 2005):

http://adsabs.harvard.edu/cgi-bin/bib_query?1995ARA\&A. .33 . 283G. 3.1

Gollub, J.P., Swinney, H.L., 1975, "Onset of turbulence in a Rotating Fluid", Phys. Rev. Lett., 35, 927-930. $\quad 1,2.6$

Grappin, R., 1986, "Onset and decay of two-dimensional magnetohydrodynamic turbulence with velocity magnetic field correlation", Phys. Fluids, 29, 2433-2443. Related online version (cited on 07 September 2005):

http://adsabs.harvard.edu/cgi-bin/bib_query?1986PhF1 . .29.2433G. 3.1 .4

Living Reviews in Solar Physics

http://www . livingreviews . org/Irsp-2005-4 
Grappin, R., Frisch, U., Pouquet, A., Leorat, J., 1982, "Alfvénic fluctuations as asymptotic states of MHD turbulence", Astron. Astrophys., 105, 6-14. Related online version (cited on 07 September 2005):

http://adsabs.harvard.edu/cgi-bin/bib_query?1982A\&A...105....6G. 3.2 .2

Grappin, R., Leorat, J., Pouquet, A., 1983, "Dependence of MHD turbulence spectra on the velocity field-magnetic field correlation", Astron. Astrophys., 126, 51-58. Related online version (cited on 07 September 2005):

http://adsabs. harvard.edu/cgi-bin/bib_query?1983A\&A ...126 _..51G. 3.1 .8

Grappin, R., Mangeney, A., Marsch, E., 1989, "On the origin of solar wind turbulence: Helios data revisited", in Turbulence and Nonlinear Dynamics in MHD Flows, (Eds.) Meneguzzi, M., Pouquet, A., Sulem, P.L., Proceedings of the Workshop, Cargèse, France, July 4-8, 1988, pp. 81-86, North-Holland, Amsterdam, Netherlands; New York, U.S.A. Related online version (cited on 07 September 2005):

http://adsabs.harvard.edu/cgi-bin/bib_query?1990JGR ...95.8197G. 3.2.1, 4.2, 13.3

Grappin, R., Mangeney, A., Marsch, E., 1990, "On the origin of solar wind MHD turbulence HELIOS data revisited", J. Geophys. Res., 95, 8197-8209. Related online version (cited on 07 September 2005):

http://adsabs.harvard.edu/cgi-bin/bib_query?1990JGR....95.8197G. 3.2, 3.2.1, 3.2.2, 6

Grappin, R., Velli, M., Mangeney, A., 1991, "Alfvénic versus standard turbulence in the solar wind", Ann. Geophys., 9, 416-426. Related online version (cited on 07 September 2005):

http://adsabs.harvard.edu/cgi-bin/bib_query?1991AnGeo...9. .416G. 3.1.8, 3.1.8, i

Grappin, R., Mangeney, A., Velli, M., 1992, "MHD turbulence: theory/simulations", in Solar Wind Seven, (Eds.) Marsch, E., Schwenn, R., Proceedings of the 3rd COSPAR Colloquium held in Goslar, Germany, 16-20 September 1991, vol. 3 of COSPAR Colloquia Series, pp. 451-456, Pergamon Press, Oxford, U.K.; New York, U.S.A. 5.1

Halmos, P.R., 1956, Lectures on Ergodic Theory, Chelsea, New York, U.S.A. 1.2

Hassler, D.M., Dammasch, I.E., Lemaire, P., Brekke, P., Curdt, W., Mason, H.E., Vial, J., Wilhelm, K., 1999, "Solar Wind Outflow and the Chromospheric Magnetic Network", Science, 283, 810-813. Related online version (cited on 07 September 2005):

http://adsabs.harvard.edu/cgi-bin/bib_query?1999Sci...283..810H. 3.1, 15

Hattori, Y., Ishizawa, A., 2001, "Characteristic time scales and energy transfer in MHD turbulence", in IUTAM Symposium on Geometry and Statistics of Turbulence, (Eds.) Kambe, T., Nakano, T., Miyauchi, T., Held in Hayama, Japan, November 1-5, 1999, vol. 59 of Fluid Mechanics and its Applications, pp. 89-94, Kluwer, Dordrecht, Netherlands; Boston, U.S.A. 7.6

Heinemann, M., Olbert, S., 1980, "Non-WKB Alfvén waves in the solar wind", J. Geophys. Res., 85, 1311-1327. Related online version (cited on 07 September 2005):

http://adsabs.harvard.edu/cgi-bin/bib_query?1980JGR....85.1311H. 4.2

Hentschel, H.G.E.F., Procaccia, I., 1983, "The infinite number of generalized dimensions of fractals and strange attractor", Physica D, 8, 435-444. Related online version (cited on 07 September 2005):

http://adsabs.harvard.edu/cgi-bin/bib_query?1983PhyD ...8..435H. 7.3 
High Altitude Observatory, H.A.O., 1991, "NASA/Marshall Solar Physics", project homepage, NASA. URL (cited on 07 September 2005):

http://science.nasa.gov/ssl/pad/solar/. 16

Horbury, T.S., Balogh, A., 1997, "Structure function measurements of the intermittent MHD turbulent cascade", Nonlinear Proc. Geophys., 4, 185-199. Related online version (cited on 07 September 2005):

http://adsabs.harvard.edu/cgi-bin/bib_query?1997NPGeo...4.185H. 8.1

Horbury, T.S., Balogh, A., 2001, "Evolution of magnetic field fluctuations in high-speed solar wind streams: Ulysses and Helios observations", J. Geophys. Res., 106, 15,929-15,940. Related online version (cited on 07 September 2005):

http://adsabs.harvard.edu/cgi-bin/bib_query?2001JGR . . 10615929H. 4.1, 50, 4.1, 4.1, $51,52,4.2,57$

Horbury, T.S., Tsurutani, B., 2001, "Ulysses measurements of waves, turbulence and discontinuities", in The Heliosphere Near Solar Minimum: The Ulysses perspective, (Eds.) Balogh, A., Marsden, R.G., Smith, E.J., Springer-Praxis Books in Astrophysics and Astronomy, pp. 167-227, Springer, London, U.K.; New York, U.S.A. 1, 4.1, 4.1

Horbury, T.S., Balogh, A., Forsyth, R.J., Smith, E.J., 1995a, "ULYSSES magnetic field observations of fluctuations within polar coronal flows", Ann. Geophys., 13, 105-107. Related online version (cited on 07 September 2005):

http://adsabs.harvard.edu/cgi-bin/bib_query?1995AnGeo..13..105H. 4.1, 47, 4.1, 48, 8.1

Horbury, T.S., Balogh, A., Forsyth, R.J., Smith, E.J., 1995b, "Anisotropy of inertial range turbulence in the polar heliosphere", Geophys. Res. Lett., 22, 3405-3408. Related online version (cited on 07 September 2005):

http://adsabs.harvard.edu/cgi-bin/bib_query?1995GeoRL . .22.3405H. 4.1

Horbury, T.S., Balogh, A., Forsyth, R.J., Smith, E.J., 1995c, "Observations of evolving turbulence in the polar solar wind", Geophys. Res. Lett., 22, 3401-3404. Related online version (cited on 07 September 2005):

http://adsabs.harvard.edu/cgi-bin/bib_query?1995GeoRL . .22.3401H. 4.1, 46, 8.1

Horbury, T.S., Balogh, A., Forsyth, R.J., Smith, E.J., 1996a, "The rate of turbulent evolution over the Sun's poles", Astron. Astrophys., 316, 333-341. Related online version (cited on 07 September 2005):

http://adsabs.harvard.edu/cgi-bin/bib_query?1996A\&A...316..333H. 4.1, 4.1, 9.2

Horbury, T.S., Balogh, A., Forsyth, R.J., Smith, E.J., 1996b, "Magnetic field signatures of unevolved turbulence in solar polar flows", J. Geophys. Res., 101, 405-414. Related online version (cited on 07 September 2005):

http://adsabs.harvard.edu/cgi-bin/bib_query?1996JGR . . 101. .405H. 4.1

Horbury, T.S., Balogh, A., Forsyth, R.J., Smith, E.J., 1997, "ULYSSES observations of intermittent heliospheric turbulence", Adv. Space Res., 19, 847-850. Related online version (cited on 07 September 2005):

http://adsabs.harvard.edu/cgi-bin/bib_query?1997AdSpR . 19. .847H. 8.1

Hoshino, M., Goldstein, M.L., 1989, "Time evolution from linear to nonlinear stages in magnetohydrodynamic parametric instabilities", Phys. Fluids B, 1, 1405-1415. 5.2

Living Reviews in Solar Physics

http: //www . livingreviews . org/lrsp-2005-4 
Huang, K., 1987, Statistical Mechanics, Wiley, New York, U.S.A., 2nd edn. 1.2

Hunt, J.C.R., Phillips, O.M., Williams, D. (Eds.), 1991, Turbulence and stochastic processes: Kolmogorov's ideas 50 years on, vol. 434 of Proc. R. Soc. London, Ser. A, The Royal Society, London, U.K. 3

Iroshnikov, P.S., 1963, "Turbulence of a Conducting Fluid in a Strong Magnetic Field", Sov. Astron., 7, 566. 2.9, 3.1.7

Katul, G.G., Hsieh, C.I., Sigmon, J., 1997, "Energy-inertial scale interaction for temperature and velocity in the unstable surface layer", Boundary-Layer Meteorol., 82, 49-80. Related online version (cited on 07 September 2005):

http://adsabs.harvard.edu/cgi-bin/bib_query?1997BoLMe..82_..49K. 9, 75

Klein, L., Bruno, R., Bavassano, B., Rosenbauer, H., 1993, "Anisotropy and minimum variance of magnetohydrodynamic fluctuations in the inner heliosphere", J. Geophys. Res., 98, 1746117466. Related online version (cited on 07 September 2005): http://adsabs .harvard.edu/cgi-bin/bib_query?1993JGR . . .9817461K. 3.1.3, 6, 6.3

Klein, L.W., 1987, Observations of Turbulence and Fluctuations in the Solar Wind, Ph.D. Thesis, Catholic University of America, Washington, U.S.A. 3.1.2

Klein, L.W., Roberts, D.A., Goldstein, M.L., 1991, "Anisotropy and minimum variance directions of solar wind fluctuations in the outer heliosphere", J. Geophys. Res., 96, 3779-3788. Related online version (cited on 07 September 2005):

http://adsabs .harvard.edu/cgi-bin/bib_query?1991JGR . . .96.3779K. 3.1.3, 9.1

Klein, L.W., Matthaeus, W.H., Roberts, D.A., Goldstein, M.L., 1992, "Evolution of spatial and temporal correlations in the solar wind - Observations and interpretation", in Solar Wind Seven, (Eds.) Marsch, E., Schwenn, R., Proceedings of the 3rd COSPAR Colloquium held in Goslar, Germany, 16-20 September 1991, vol. 3 of COSPAR Colloquia Series, pp. 197-200, Pergamon Press, Oxford, U.K.; New York, U.S.A. Related online version (cited on 07 September 2005): http://adsabs.harvard.edu/cgi-bin/bib_query?1992sws...coll...197K. 3.1 .2

Kolmogorov, A.N., 1941, "The local structure turbulence in incompressible viscous fluids for very large Reynolds numbers", Dokl. Akad. Nauk. SSSR, 30, 301-305. Reprinted in Proc. R. Soc. London, Ser. A 434 (1991) 9-13. 1, 3, 6, 2.10, 3.1.7, 7.1

Kraichnan, R.H., 1965, "Inertial Range Spectrum of Hydromagnetic Turbulence", Phys. Fluids, 8, 1385-1387. 1, 2.9, 7, 3.1.7, 3.1.8, 8.1, 8.1

Kraichnan, R.H., 1974, "On Kolmogorov's Inertial-Range Theories", J. Fluid Mech., 62, 305-330. Related online version (cited on 07 September 2005): http://adsabs.harvard.edu/cgi-bin/bib_query?1974JFM....62. .305K. 3.1.7, 7.3

Krause, F., Rädler, K.H., 1980, Mean field magnetohydrodynamics and dynamo theory, AkademieVerlag, Berlin, Germany. 3.1.8

Landau, L.D., Lifshitz, E.M., 1971, Physique théorique, Vol. 6: Mécanique des fluides, Editions MIR, Moscow, U.S.S.R. 1, 2.6, 7.1

Lorenz, E.N., 1963, "Deterministic Nonperiodic Flow", J. Atmos. Sci., 20, $130 . \quad$ 1, 2.6

Malara, F., Velli, M., 1996, "Parametric instability of a large-amplitude nonmonochromatic Alfvén wave", Phys. Plasmas, 3, 4427-4433. Related online version (cited on 07 September 2005): http://adsabs.harvard.edu/cgi-bin/bib_query?1996PhPl _...3.4427M. 5.2 
Malara, F., Veltri, P., Carbone, V., 1992, "Competition among nonlinear effects in tearing instability saturation", Phys. Fluids B, 4, 3070. Related online version (cited on 07 September 2005): http://adsabs.harvard.edu/cgi-bin/bib_query?1992PhFlB . .4.3070M. 5.1

Malara, F., Primavera, L., Veltri, P., 1996, "Compressive fluctuations generated by time evolution of Alfvénic perturbations in the solar wind current sheet", J. Geophys. Res., 101, 21 597-21617. Related online version (cited on 07 September 2005): http: //adsabs.harvard.edu/cgi-bin/bib_query?1996JGR . . 10121597M. 5.1

Malara, F., Primavera, L., Veltri, P., 2000, "Nonlinear evolution of parametric instability of a large-amplitude nonmonochromatic Alfvén wave", Phys. Plasmas, 7, 2866-2877. Related online version (cited on 07 September 2005):

http://adsabs.harvard.edu/cgi-bin/bib_query?2000PhPl...7.2866M. 3.2.2, 5.1, 5.2

Malara, F., Primavera, L., Veltri, P., 2001, "Parametric Instability of a Broad-band Alfvén Wave: Nonlinear Evolution and Saturation", in Recent insights into the physics of the sun and heliosphere: Highlights from SOHO and other space missions, (Eds.) Brekke, P., Fleck, B., Gurman, J.B., Proceedings of the 24th General Assembly of the IAU held at Manchester, United Kingdom, 7-11 August 2000 (IAU Symposium 203, pp. 511-513, Astronomical Society of the Pacific, San Francisco, U.S.A. Related online version (cited on 07 September 2005):

http://adsabs.harvard.edu/cgi-bin/bib_query?2001IAUS . 203..511M. 3.2.2, 4.1, 5.2, 61, 5.2, 62

Malara, F., Primavera, L., Veltri, P., 2002, "Parametric instability of Alfvénic fluctuations in highlatitude solar wind", in Solspa 2001, (Ed.) Sawaya-Lacoste, H., Proceedings of the Second Solar Cycle and Space Weather Euroconference, 24-29 September 2001, Vico Equense, Italy, vol. SP-477 of ESA Conference Proceedings, pp. 309-312, ESA Publications Division, Noordwijk, Netherlands. Related online version (cited on 07 September 2005):

http://adsabs.harvard.edu/cgi-bin/bib_query?2002scsw.conf . 309M. 3.2.2, 4.1

Mariani, F., Ness, N.F., Burlaga, L.F., Bavassano, B., Villante, U., 1978, "The large-scale structure of the interplanetary magnetic field between 1 and $0.3 \mathrm{AU}$ during the primary mission of HELIOS 1", J. Geophys. Res., 83, 5161-5166. Related online version (cited on 07 September 2005): http://adsabs.harvard.edu/cgi-bin/bib_query?1978JGR . . .83.5161M. 4.1

Marsch, E., 1992, "Introduction to kinetic physics, waves and turbulence in the solar wind", in Solar Wind Seven, (Eds.) Marsch, E., Schwenn, R., Proceedings of the 3rd COSPAR Colloquium held in Goslar, Germany, 16-20 September 1991, vol. 3 of COSPAR Colloquia Series, pp. 499-504, Pergamon Press, Oxford, U.K.; New York, U.S.A. 7

Marsch, E., Liu, S., 1993, "Structure functions and intermittency of velocity fluctuations in the inner solar wind", Ann. Geophys., 11, 227-238. Related online version (cited on 07 September 2005):

http://adsabs.harvard.edu/cgi-bin/bib_query?1993AnGeo..11.227M. 8.1, 9.1, 9.1, 9.2

Marsch, E., Mangeney, A., 1987, "Ideal MHD equations in terms of compressive Elsässer variables", J. Geophys. Res., 92, 7363-7367. Related online version (cited on 07 September 2005): http://adsabs.harvard.edu/cgi-bin/bib_query?1987JGR . . .92.7363M. 2.5, 3.2

Marsch, E., Tu, C.-Y., 1989, "Dynamics of correlation functions with Elsässer variables for inhomogeneous MHD turbulence", J. Plasma Phys., 41, 479-491. Related online version (cited on 07 September 2005):

http://adsabs.harvard.edu/cgi-bin/bib_query?1989JP1Ph..41..479M. 2.5, 13.3

Living Reviews in Solar Physics

http://www. livingreviews.org/lrsp-2005-4 
Marsch, E., Tu, C.-Y., 1990a, "On the radial evolution of MHD turbulence in the inner heliosphere", J. Geophys. Res., 95, 8211-8229. Related online version (cited on 07 September 2005): http://adsabs.harvard.edu/cgi-bin/bib_query?1990JGR ...95.8211M. 3.1.3, 3.1.8, 32, $37,4.1,4.2,5.1$

Marsch, E., Tu, C.-Y., 1990b, "Spectral and spatial evolution of compressible turbulence in the inner solar wind", J. Geophys. Res., 95, 11945-11956. Related online version (cited on 07 September 2005):

http://adsabs.harvard.edu/cgi-bin/bib_query?1990JGR....9511945M. 3.1.2, 6, 64

Marsch, E., Tu, C.-Y., 1993a, "Modeling results on spatial transport and spectral transfer of solar wind Alfvénic turbulence", J. Geophys. Res., 98, 21 045-21 059. Related online version (cited on 07 September 2005):

http://adsabs.harvard.edu/cgi-bin/bib_query?1993JGR . . .9821045M. 3.1.8, 3.2.2, 4.1, $5.1,60,6.1,6.1$

Marsch, E., Tu, C.-Y., 1993b, "Correlations between the fluctuations of pressure, density, temperature and magnetic field in the solar wind", Ann. Geophys., 11, 659-677. Related online version (cited on 07 September 2005):

http://adsabs.harvard.edu/cgi-bin/bib_query?1993AnGeo..11..659M. 5.1, 66

Marsch, E., Tu, C.-Y., 1994, "Non-Gaussian probability distributions of solar wind fluctuations", Ann. Geophys., 12, 1127-1138. Related online version (cited on 07 September 2005):

http://adsabs.harvard.edu/cgi-bin/bib_query?1994AnGeo..12.1127M. 8.2, 9.1, 9.2

Marsch, E., Tu, C.-Y., 1996, "Spatial evolution of the magnetic field spectral exponent in the solar wind: Helios and Ulysses comparison", J. Geophys. Res., 101, 11149-11 152. Related online version (cited on 07 September 2005):

http://adsabs.harvard.edu/cgi-bin/bib_query?1996JGR...10111149M. 4.1, 4.1, 48

Marsch, E., Tu, C.-Y., 1997, "Intermittency, non-Gaussian statistics and fractal scaling of MHD fluctuations in the solar wind", Nonlinear Proc. Geophys., 4, 101-124. Related online version (cited on 07 September 2005):

http://adsabs.harvard.edu/cgi-bin/bib_query?1997NPGeo...4.101M. 8.1, 8.1

Marsch, E., Tu, C.-Y., Rosenbauer, H., 1996, "Multifractal scaling of the kinetic energy flux in solar wind turbulence", Ann. Geophys., 14, 259-269. Related online version (cited on 07 September 2005):

http://adsabs.harvard.edu/cgi-bin/bib_query?1995sowi.conf...81M. 8.1

Matthaeus, W.H., 1986, "The Alfvén effect reconsidered", unknown status. Paper presented at 1986 Sherwood Controlled Fusion Theory Conference, Courant Institute of Mathematical Sciences, New York. 3.1.8

Matthaeus, W.H., Brown, M.R., 1988, "Nearly incompressible magnetohydrodynamics at low Mach number", Phys. Fluids, 31, 3634-3644. Related online version (cited on 07 September 2005): http://adsabs.harvard.edu/cgi-bin/bib_query?1988PhFl_..31.3634M. 6.1

Matthaeus, W.H., Goldstein, M.L., 1982a, "Stationarity of magnetohydrodynamic fluctuations in the solar wind", J. Geophys. Res., 87, 10347-10 354. Related online version (cited on 07 September 2005):

http://adsabs.harvard.edu/cgi-bin/bib_query?1982JGR . . 8710347M. $\quad 3.1 .2,13$ 
Matthaeus, W.H., Goldstein, M.L., 1982b, "Measurement of the rugged invariants of magnetohydrodynamic turbulence in the solar wind", J. Geophys. Res., 87, 6011-6028. Related online version (cited on 07 September 2005):

http://adsabs.harvard.edu/cgi-bin/bib_query?1982JGR . . .87.6011M. 3.1.5, 3.1.6, 27, $3.1 .8,3.1 .8,98,13.2$

Matthaeus, W.H., Goldstein, M.L., 1986, "Low-frequency 1/f noise in the interplanetary magnetic field", Phys. Rev. Lett., 57, 495-498. Related online version (cited on 07 September 2005): http://adsabs.harvard.edu/cgi-bin/bib_query?1986PhRvL. .57 .495M. 3.1 .2

Matthaeus, W.H., Goldstein, M.L., Roberts, D.A., 1990, "Evidence for the presence of quasitwo-dimensional nearly incompressible fluctuations in the solar wind", J. Geophys. Res., 95, 20 673-20 683. Related online version (cited on 07 September 2005):

http://adsabs.harvard.edu/cgi-bin/bib_query?1990JGR....9520673M. 3.1.5, 26, 3.1.5, 3.2 .1

Matthaeus, W.H., Klein, L.W., Ghosh, S., Brown, M.R., 1991, "Nearly incompressible magnetohydrodynamics, pseudosound, and solar wind fluctuations", J. Geophys. Res., 96, 5421-5435. Related online version (cited on 07 September 2005):

http://adsabs.harvard.edu/cgi-bin/bib_query?1991JGR . . .96.5421M. 6.1, 6.2

McComas, D.J., Barraclough, B.L., Gosling, J.T., Hammond, C.M., Phillips, J.L., Neugebauer, M., Balogh, A., Forsyth, R.J., 1995, "Structures in the polar solar wind: Plasma and field observations from Ulysses", J. Geophys. Res., 100, 19 893-19 902. Related online version (cited on 07 September 2005):

http://adsabs.harvard.edu/cgi-bin/bib_query?1995JGR . . 10019893M. 6.2

McComas, D.J., Hoogeveen, G.W., Gosling, J.T., Phillips, J.L., Neugebauer, M., Balogh, A., Forsyth, R.J., 1996, "Ulysses observations of pressure-balance structures in the polar solar wind", Astron. Astrophys., 316, 368-373. Related online version (cited on 07 September 2005):

http://adsabs.harvard.edu/cgi-bin/bib_query?1996A\&A...316 . 368M. 6.2

McComas, D.J., Bame, S.J., Barraclough, B.L., Feldman, W.C., Funsten, H.O., Gosling, J.T., Riley, P., Skoug, R.M., Balogh, A., Forsyth, R.J., Goldstein, B.E., Neugebauer, M., 1998, "Ulysses' return to the slow solar wind", Geophys. Res. Lett., 25, 1-4. Related online version (cited on 07 September 2005):

http://adsabs.harvard.edu/cgi-bin/bib_query?1998GeoRL..25...1M. 4

McComas, D.J., Elliott, H.A., Schwadron, N.A., Gosling, J.T., Skoug, R.M., Goldstein, B.E., 2003, "The three-dimensional solar wind around solar maximum", Geophys. Res. Lett., 30, 241. Related online version (cited on 07 September 2005):

http://adsabs.harvard.edu/cgi-bin/bib_query?2003AIPC..679...33M. 4, 43

McComb, W.D., 1990, The Physics of Fluid Turbulence, vol. 25 of Oxford Engineering Science Series, Oxford University Press, Oxford, U.K.; New York, U.S.A. 2

McCracken, K.G., Ness, N.F., 1966, "The collimation of cosmic rays by the interplanetary magnetic field", J. Geophys. Res., 71, 3315-3325. 6.1

Meneveau, C., 1991, "Analysis of turbulence in the orthonormal wavelet rapresentation", J. Fluid Mech., 232, 469-520. Related online version (cited on 07 September 2005):

http://adsabs.harvard.edu/cgi-bin/bib_query?1991JFM . . 232 . .469M. 7.3, 7.4, 8.1

Living Reviews in Solar Physics

http: //www. livingreviews . org/lrsp-2005-4 
Meneveau, C., Sreenivasan, K.R., 1987a, "Simple multifractal cascade model for fully developed turbulence", Phys. Rev. Lett., 59, 1424-1427. Related online version (cited on 07 September 2005):

http://adsabs.harvard.edu/cgi-bin/bib_query?1987PhRvL. .59.1424M. 8.1, 8.1

Meneveau, C., Sreenivasan, K.R., 1987b, "Simple multifractal cascade model for fully developed turbulence", Phys. Rev. Lett., 59, 1424-1427. Related online version (cited on 07 September 2005):

http://adsabs.harvard.edu/cgi-bin/bib_query?1987PhRvL. .59.1424M. 8.1

Mininni, P.D., Gómez, D.O., Mahajan, S.M., 2003a, "Dynamo Action in Magnetohydrodynamics and Hall-Magnetohydrodynamics", Astrophys. J., 587, 472-481. Related online version (cited on 07 September 2005):

http://adsabs.harvard.edu/cgi-bin/bib_query?2003ApJ. ..587. .472M. 3.1.6, 28, 3.1.8, 3.1 .8

Mininni, P.D., Gómez, D.O., Mahajan, S.M., 2003b, "Role of the Hall Current in Magnetohydrodynamic Dynamos", Astrophys. J., 584, 1120-1126. Related online version (cited on 07 September 2005):

http://adsabs.harvard.edu/cgi-bin/bib_query?2003ApJ ...584.1120M. 3.1.8

Mjolhus, E., 1976, "On the modulational instability of hydromagnetic waves parallel to the magnetic field", J. Plasma Phys., 16, 321-334. Related online version (cited on 07 September 2005): http://adsabs.harvard.edu/cgi-bin/bib_query?1976JP1Ph..16..321M. 4

Moffat, H.K., 1978, Magnetic field generation in electrically conducting fluids, Cambridge University Press, Cambridge, U.K.; New York, U.S.A. 3.1.6

Monin, A.S., Yaglom, A.M., 1975, Statistical fluid mechanics - Mechanics of turbulence, Vol. 2, MIT Press, Cambridge, U.S.A. 4.1

Montgomery, D., 1982, "Major disruptions, inverse cascades, and the Strauss equations", Phys. Scripta, 2, 83-88. Related online version (cited on 07 September 2005):

http://adsabs.harvard.edu/cgi-bin/bib_query?1982PhST ...2. . .83M. $\quad 3.1 .5,3.1 .5$

Montgomery, D., 1983, "Theory of hydromagnetic turbulence", in Solar Wind Five, (Ed.) Neugebauer, M., Proceedings of a conference held in Woodstock, Vermont, November 1-5 1982, vol. 2280 of NASA Conference Publication, pp. 107-130, NASA, Washington, U.S.A. 13.2

Montgomery, D., Brown, M.R., Matthaeus, W.H., 1987, "Density fluctuation spectra in magnetohydrodynamic turbulence", J. Geophys. Res., 92, 282-284. Related online version (cited on 07 September 2005):

http://adsabs.harvard.edu/cgi-bin/bib_query?1987JGR....92..282M. 6.1

Novikov, E.A., 1969, "Scale similarity for random fields", Sov. Phys. Dokl., 14, 104-107. 7.4

Ohkitani, K., Yamada, M., 1989, "Temporal intermittency in the energy cascade process and local Lyapunov analysis in fully-developed model turbulence", Prog. Theor. Phys., 89, 329-341. Related online version (cited on 07 September 2005):

http://adsabs.harvard.edu/cgi-bin/bib_query?1989PThPh..81..3290. 2.7

Okkels, F., 1997, The Intermittent Dynamics in Turbulent Shell Models, University of Copenhagen, Copenhagen, Denmark. Related online version (cited on 07 September 2005):

http://www.nbi.dk/CATS/theses.html. 7.6, 7.6 
Onorato, M., Camussi, R., Iuso, G., 2000, "Anomalous scaling and bursting process in an experimental turbulent channel flow", Phys. Rev. E, 61, 1447-1460. 14

Oughton, S., 1993, Transport of solar wind fluctuations: A turbulence approach, Ph.D. Thesis, Delaware University, Wilmington, U.S.A. Related online version (cited on 07 September 2005): http://adsabs.harvard.edu/cgi-bin/bib_query?1993PhDT . . . . ...120. 3.1 .5

Oughton, S., Matthaeus, W.H., 1992, "Evolution of solar wind fluctuations and the influence of turbulent 'mixing"', in Solar Wind Seven, (Eds.) Marsch, E., Schwenn, R., Proceedings of the 3rd COSPAR Colloquium held in Goslar, Germany, 16-20 September 1991, vol. 3 of COSPAR Colloquia Series, pp. 523-526, Pergamon Press, Oxford, U.K.; New York, U.S.A. 2.5

Oughton, S., Priest, E., Matthaeus, W.H., 1994, "The influence of a mean magnetic field on threedimensional MHD turbulence", J. Fluid Mech., 280, 95-117. Related online version (cited on 07 September 2005):

http://adsabs.harvard.edu/cgi-bin/bib_query?1994JFM . . . 280 . . $950 . \quad 3.1 .4$

Pagel, C., Balogh, A., 2002, "Intermittency in the solar wind: A comparison between solar minimum and maximum using Ulysses data", J. Geophys. Res., 107, 1178. Related online version (cited on 07 September 2005):

http: //adsabs.harvard.edu/cgi-bin/bib_query?2002JGRA.107h.SSH6P. 9.2

Pagel, C., Balogh, A., 2003, "Radial dependence of intermittency in the fast polar solar wind magnetic field using Ulysses", J. Geophys. Res., 108, 1012. Related online version (cited on 07 September 2005):

http://adsabs.harvard.edu/cgi-bin/bib_query?2003JGRA.108a.SSH2P. 9.2, 96

Paladin, G., Vulpiani, A., 1987, "Anomalous scaling laws in multifractal objects", Phys. Rep., 156, 147-225. Related online version (cited on 07 September 2005):

http://adsabs.harvard.edu/cgi-bin/bib_query?1987PhR . .156 . 147P. 8.1

Parker, E.N., 1963, "Theory of Solar Wind", in Proceedings of the International Conference on Cosmic Rays, Vol. 1: Solar Particles and Sun-Earth Relations, Held in December 1963 at Jaipur, India, p. 175, Tata Institute of Fundamental Research, Bombay, India. 6.1

Parker, E.N., 1980, "Book note: Cosmical magnetic fields: Their origin and their activity", Astron. Quart., 3, 201-201. 3.1 .3

Paschmann, G., Fazakerley, A.N., Schwartz, S., 1998, "Moments of Plasma Velocity Distributions", in Analysis Methods for Multi-Spacecraft Data, (Eds.) Paschmann, G., Daly, P.W., vol. SR-001 of ISSI Scientific Report, pp. 125-158, ESA Publication Divisions for ISSI, Noordwijk, Netherlands. Related online version (cited on 07 September 2005):

http://www.issi.unibe.ch/publi_scirep.html. 16.3

Pfaff, R.F., Borovsky, J.E., Young, D.T. (Eds.), 1998a, Measurement Techniques in Space Plasmas, Vol. 1: Particles, vol. 102 of Geophysical Monograph, American Geophysical Union, Washington, U.S.A. 16

Pfaff, R.F., Borovsky, J.E., Young, D.T. (Eds.), 1998b, Measurement Techniques in Space Plasmas, Vol. 2: Fields, vol. 102 of Geophysical Monograph, American Geophysical Union, Washington, U.S.A. $\quad 16,16.4$

Pierrehumbert, R.T., 1999, "Huascaran $\delta^{18} \mathrm{O}$ as an indicator of tropical climate during the Last Glacial Maximum", Geophys. Res. Lett., 26, 1345-1348. Related online version (cited on 07 September 2005):

http://adsabs.harvard.edu/cgi-bin/bib_query?1999GeoRL . 26.1345P. 9.2

Living Reviews in Solar Physics

http: //www. livingreviews . org/lrsp-2005-4 
Politano, H., Pouquet, A., 1995, "Model of intermittency in magnetohydrodynamic turbulence", Phys. Rev. E, 52, 636-641. Related online version (cited on 07 September 2005):

http://adsabs.harvard.edu/cgi-bin/bib_query?1995PhRvE. 52 .636P. 2.10, 2.10, 7.4

Politano, H., Pouquet, A., 1998, "von Kármán-Howarth equation for magnetohydrodynamics and its consequences on third-order longitudinal structure and correlation functions", Phys. Rev. E, 57, R21-R25. Related online version (cited on 07 September 2005):

http://adsabs.harvard.edu/cgi-bin/bib_query?1998PhRvE..57 ..21P. 7.4

Politano, H., Pouquet, A., Carbone, V., 1998a, "Determination of anomalous exponents of structure functions in two-dimensional magnetohydrodynamic turbulence", Europhys. Lett., 43, 516. Related online version (cited on 07 September 2005):

http://adsabs.harvard.edu/cgi-bin/bib_query?1998EL ....43..516P. 2.10

Politano, H., Pouquet, A., Carbone, V., 1998b, "Determination of anomalous exponents of structure functions in two-dimensional magnetohydrodynamic turbulence", Europhys. Lett., 43, 516521. Related online version (cited on 07 September 2005):

http://adsabs.harvard.edu/cgi-bin/bib_query?1998EL ....43..516P. 9, 3, 9

Polygiannakis, J.M., Moussas, X., Quenby, J.J., Smith, E.J., 1994, "Spectral polarization analysis of the interplanetary magnetic field fluctuations", Solar Phys., 149, 381-389. Related online version (cited on 07 September 2005):

http://adsabs.harvard.edu/cgi-bin/bib_query?1994SoPh..149...381P. 3.1.6

Pope, S.B., 2000, Turbulent flows, Cambridge University Press, Cambridge, U.K.; New York, U.S.A. $\quad 2,9$

Pouquet, A., 1993, "Magnetohydrodynamic Turbulence", in Les Houches Summer School on Astrophysical Fluid Dynamics, (Eds.) Zahn, J.-P., Zinn-Justin, J., Proceedings of the Les Houches Summer School, Course XLVII, 29 June - 31 July, 1987, p. 139, Elsevier, Amsterdam, Netherlands. 5

Pouquet, A., 1996, "Turbulence, Statistics and Structures: an Introduction", in Plasma Astrophysics, (Eds.) Chiuderi, C., Einaudi, G., Lectures held at the Astrophysics School VII in San Miniato, Italy, 3-14 October 1994, vol. 468 of Lecture Notes in Physics, p. 163, Springer, Berlin, Germany; New York, U.S.A. 5

Pouquet, A., Frisch, U., Leorat, J., 1976, "Strong MHD helical turbulence and the nonlinear dynamo effect", J. Fluid Mech., 77, 321-354. Related online version (cited on 07 September 2005):

http://adsabs.harvard.edu/cgi-bin/bib_query?1976JFM....77...321P. 3.1.6

Primavera, L., Malara, F., Veltri, P., 2003, "Parametric instability in the solar wind: numerical study of the nonlinear evolution", in Solar Wind Ten, (Eds.) Velli, M., Bruno, R., Malara, F., Proceedings of the Tenth International Solar Wind Conference, Pisa, Italy, 17-21 June 2002, vol. 679 of AIP Conference Proceedings, pp. 505-508, American Institute of Physics, Melville, U.S.A. $\quad 3.2 .2,4.1$

Reisenfeld, D.B., McComas, D.J., Steinberg, J.T., 1999, "Evidence of a solar origin for pressure balance structures in the high-latitude solar wind", Geophys. Res. Lett., 26, 1805-1808. Related online version (cited on 07 September 2005):

http://adsabs.harvard.edu/cgi-bin/bib_query?1999GeoRL..26.1805R. 6.2 
Reynolds, O., 1883, "An experimental investigation of the circumstances which determine whether the motion of water shall be direct or sinuous, and the law of resistance in parallel channels", Philos. Trans. R. Soc. London, 174, 935-982. Related online version (cited on 07 September 2005):

http://www.eng.man.ac.uk/historic/reynolds/oreynB.htm. 8

Roberts, D.A., 1989, "Interplanetary observational constraints on Alfvén wave acceleration of the solar wind", J. Geophys. Res., 94, 6899-6905. Related online version (cited on 07 September 2005):

http://adsabs.harvard.edu/cgi-bin/bib_query?1989JGR . . .94.6899R. 4.1

Roberts, D.A., 1992, "Observation and simulation of the radial evolution and stream structure of solar wind turbulence", in Solar Wind Seven, (Eds.) Marsch, E., Schwenn, R., Proceedings of the 3rd COSPAR Colloquium held in Goslar, Germany, 16-20 September 1991, vol. 3 of COSPAR Colloquia Series, pp. 533-538, Pergamon Press, Oxford, U.K.; New York, U.S.A. 3.1.2, 3.1.8, 6

Roberts, D.A., Goldstein, M.L., 1988, "Simulation of Interplanetary Dynamical Processes", in Proceedings of the Third International Conference on Supercomputing, (Eds.) Kartashev, L.P., Kartashev, S.I., p. 370, International Supercomputing Institute, St. Petersburg, U.S.A. 5.1

Roberts, D.A., Goldstein, M.L., Klein, L.W., Matthaeus, W.H., 1987a, "The nature and evolution of magnetohydrodynamic fluctuations in the solar wind: Voyager observations", J. Geophys. Res., 92, 11 021-11 040. Related online version (cited on 07 September 2005):

http://adsabs.harvard.edu/cgi-bin/bib_query?1987JGR . . .9211021R. 3.1.7, 3.1.8, 4.1, $5.1,13.3$

Roberts, D.A., Goldstein, M.L., Klein, L.W., Matthaeus, W.H., 1987b, "Origin and evolution of fluctuations in the solar wind: HELIOS observations and Helios-Voyager comparisons", J. Geophys. Res., 92, 12 023-12 035. Related online version (cited on 07 September 2005):

http://adsabs.harvard.edu/cgi-bin/bib_query?1987JGR. . .9212023R. 3.1.8, 31, 3.1.8, $5.1,6,13.3$

Roberts, D.A., Goldstein, M.L., Klein, L.W., 1990, "The amplitudes of interplanetary fluctuations - Stream structure, heliocentric distance, and frequency dependence", J. Geophys. Res., 95, 4203-4216. Related online version (cited on 07 September 2005):

http://adsabs.harvard.edu/cgi-bin/bib_query?1990JGR . . .95.4203R. 3.1.8, ii, 4.2

Roberts, D.A., Ghosh, S., Goldstein, M.L., Matthaeus, W.H., 1991, "Magnetohydrodynamic simulation of the radial evolution and stream structure of solar-wind turbulence", Phys. Rev. Lett., 67, 3741-3744. Related online version (cited on 07 September 2005): http://adsabs.harvard.edu/cgi-bin/bib_query?1991PhRvL. .67.3741R. 5.1, 59, 5.1, 6

Roberts, D.A., Goldstein, M.L., Matthaeus, W.H., Ghosh, S., 1992, "Velocity shear generation of solar wind turbulence", J. Geophys. Res., 97, 17115. Related online version (cited on 07 September 2005):

http://adsabs.harvard.edu/cgi-bin/bib_query?1992JGR....9717115R. 3.1.8, 4.1, 5.1, $5.1,6$

Ruelle, D., Takens, F., 1971, "On the nature of turbulence”, Commun. Math. Phys., 20, 167.1, $2.6,4$

Ruíz-Chavarría, G., Baudet, C., Ciliberto, S., 1995, "Extended Self Similarity of passive scalars in fully developed turbulence", Europhys. Lett., 32, 319. 1, ii

Living Reviews in Solar Physics

http://www . livingreviews . org/Irsp-2005-4 
Russell, C.T., 1972, "Comments on the Measurement of Power Spectra of the Interplanetary Magnetic Field", in Solar Wind, (Eds.) Sonett, C.P., Coleman, P.J., Wilcox, J.M., Proceedings of a conference held March 21-26, 1971, at the Asilomar Conference Grounds, Pacific Grove, Calif., pp. 365-374, NASA, Washington, U.S.A. 3.1.1, 22

Ruzmaikin, A., Lyannaya, I.P., Styashkin, V.A., Eroshenko, E., 1993, "The spectrum of the interplanetary magnetic field near 1.3 AU", J. Geophys. Res., 98, 13 303-13 306. Related online version (cited on 07 September 2005):

http://adsabs.harvard.edu/cgi-bin/bib_query?1993JGR....9813303R. 4.1

Ruzmaikin, A.A., Feynman, J., Goldstein, B.E., Smith, E.J., Balogh, A., 1995, "Intermittent turbulence in solar wind from the south polar hole", J. Geophys. Res., 100, 3395-3403. Related online version (cited on 07 September 2005):

http://adsabs .harvard.edu/cgi-bin/bib_query?1995JGR . .100.3395R. 4.1, 8.1

Sagdeev, R.Z., Galeev, A.A., 1969, Nonlinear Plasma Theory, Benjamin, New York, U.S.A. 5.2

Saur, J., Politano, H., Pouquet, A., Matthaeus, W.H., 2002, "Evidence for weak MHD turbulence in the middle magnetosphere of Jupiter", Astron. Astrophys., 386, 699-708. Related online version (cited on 07 September 2005):

http://adsabs.harvard.edu/cgi-bin/bib_query?2002A\&A...386..699S. 3.1 .5

Saur, J., Pouquet, A., Matthaeus, W.H., 2003, "Correction to "An acceleration mechanism for the generation of the main auroral oval on Jupiter"", Geophys. Res. Lett., 30, 19-22. Related online version (cited on 07 September 2005):

http://adsabs.harvard.edu/cgi-bin/bib_query?2003GeoRL. .30m.. 19S. 3.1.5

Scarf, F.L., Wolfe, J.H., Silva, R.W., 1967, "A Plasma Instability Associated with Thermal Anisotropies in the Solar Wind", J. Geophys. Res., 72, 993-999. Related online version (cited on 07 September 2005):

http://adsabs.harvard.edu/cgi-bin/bib_query?1967JGR . ...72..993S. 3.1.1

Schertzer, D., Lovejoy, S., Schmitt, F., Chigirinskaya, Y., Marsan, D., 1997, "Multifractal Cascade Dynamics and Turbulent Intermittency", Fractals, 5, 427-471. 10

She, Z.-S., Leveque, E., 1994, "Universal scaling laws in fully developed turbulence", Phys. Rev. Lett., 72, 336-339. Related online version (cited on 07 September 2005):

http://adsabs.harvard.edu/cgi-bin/bib_query?1994PhRvL..72..336S. 7.4

Shebalin, J.V., Matthaeus, W.H., Montgomery, D., 1983, "Anisotropy in MHD turbulence due to a mean magnetic field", J. Plasma Phys., 29, 525-547. 3.1.4, 3.1.4, 3.1.5, 3.1.5

Siscoe, G.L., Davis, L., Coleman, P.J., Smith, E.J., Jones, D.E., 1968, "Power Spectra and Discontinuities of the Interplanetary Magnetic Field: Mariner 4", J. Geophys. Res., 73, 61-99. Related online version (cited on 07 September 2005):

http://adsabs.harvard.edu/cgi-bin/bib_query?1968JGR...73..61S. 6.1

Smith, C.W., 2003, "The Geometry of Turbulent Magnetic Fluctuations at High Heliograpahic Latitudes", in Solar Wind Ten, (Eds.) Velli, M., Bruno, R., Malara, F., Proceedings of the Tenth International Solar Wind Conference, Pisa, Italy, 17-21 June 2002, vol. 679 of AIP Conference Proceedings, pp. 413-416, American Institute of Physics, Melville, U.S.A. 3.1.5

Smith, C.W., Goldstein, M.L., Matthaeus, W.H., 1983, "Turbulence analysis of the Jovian upstream wave phenomenon", J. Geophys. Res., 88, 5581-5593. Related online version (cited on 07 September 2005):

http://adsabs.harvard.edu/cgi-bin/bib_query?1983JGR . . .88.5581S. 3.1.6 
Smith, C.W., Goldstein, M.L., Matthaeus, W.H., Viñas, A.F., 1984, "Erratum: Correction to "Turbulence analysis of the Jovian upstream 'wave' phenomenon"”, J. Geophys. Res., 89, 91599160. 3.1.6

Smith, E.J., Balogh, A., Neugebauer, M., McComas, D.J., 1995, "Ulysses observations of Alfvén waves in the southern and northern solar hemispheres", Geophys. Res. Lett., 22, 3381-3384. $4.1,44,4.1$

Solodyna, C.V., Belcher, J.W., 1976, "On the minimum variance direction of magnetic field fluctuations in the azimuthal velocity structure of the solar wind", Geophys. Res. Lett., 3, 565-568. Related online version (cited on 07 September 2005):

http://adsabs.harvard.edu/cgi-bin/bib_query?1976GeoRL . . 3. .565S. 3.1.3, 3.1.8

Sonnerup, B.U.O., Cahill, L.J., 1967, "Magnetopause Structure and Attitude from Explorer 12 Observations", J. Geophys. Res., 72, 171. 3.1.3, 15.1

Sorriso-Valvo, L., Carbone, V., Veltri, P., Consolini, G., Bruno, R., 1999, "Intermittency in the solar wind turbulence through probability distribution functions of fluctuations", Geophys. Res. Lett., 26, 1801-1804. Related online version (cited on 07 September 2005): http://adsabs.harvard.edu/cgi-bin/bib_query?1999GeoRL..26.1801S. 8.2, 84, 85, 86

Sorriso-Valvo, L., Carbone, V., Giuliani, P., Veltri, P., Bruno, R., Antoni, V., Martines, E., 2001, "Intermittency in plasma turbulence", Planet. Space Sci., 49, 1193-1200. Related online version (cited on 07 September 2005):

http://adsabs.harvard.edu/cgi-bin/bib_query?2001P\&SS ..49.1193S. 8.2

Tennekes, H., Wyngaard, J., 1972, "The intermittent small-scale structure of turbulence: Dataprocessing hazards", J. Fluid Mech., 55, 93. Related online version (cited on 07 September 2005): http://adsabs.harvard.edu/cgi-bin/bib_query?1972JFM...55...93T. 9

Thieme, K.M., Schwenn, R., Marsch, E., 1989, "Are structures in high-speed streams signatures of coronal fine structures?", Adv. Space Res., 9, 127-130. Related online version (cited on 07 September 2005):

http://adsabs.harvard.edu/cgi-bin/bib_query?1989AdSpR...9.127T. 6.1, 65, 6.1, 9

Ting, A.C., Matthaeus, W.H., Montgomery, D., 1986, "Turbulent relaxation processes in magnetohydrodynamics", Phys. Fluids, 29, 3261-3274. Related online version (cited on 07 September 2005):

http://adsabs.harvard.edu/cgi-bin/bib_query?1986PhFl_..29.3261T. 5.1

Tsurutani, B.T., Smith, E.J., 1979, "Interplanetary discontinuities - Temporal variations and the radial gradient from 1 to 8.5 AU", J. Geophys. Res., 84, 2773-2787. Related online version (cited on 07 September 2005):

http://adsabs.harvard.edu/cgi-bin/bib_query?1979JGR...84.2773T. 9

Tu, C.-Y., 1987a, "A Self-Consistent Two-Time Scale Solar Wind Model", in Solar Wind Six, (Eds.) Pizzo, V.J., Holzer, T., Sime, D.G., Proceedings of the Sixth International Solar Wind Conference held 23-28 August, 1987 at YMCA of the Rockies, Estes Park, Colorado, vol. 306 of NCAR Technical Notes, p. 112, Natl. Cent. for Atmos. Res., Boulder, U.S.A. 3.1.2

Tu, C.-Y., 1987b, "A solar wind model with the power spectrum of Alfvénic fluctuations", Solar Phys., 109, 149-186. Related online version (cited on 07 September 2005):

http://adsabs.harvard.edu/cgi-bin/bib_query?1987SoPh..109..149T. 3.1 .2

Living Reviews in Solar Physics

http: //www. livingreviews.org/lrsp-2005-4 
Tu, C.-Y., 1988, "The damping of interplanetary Alfvénic fluctuations and the heating of the solar wind", J. Geophys. Res., 93, 7-20. Related online version (cited on 07 September 2005): http://adsabs.harvard.edu/cgi-bin/bib_query?1988JGR ...93_...7T. 7, 3.1.2, 8.1

Tu, C.-Y., Marsch, E., 1990a, "Transfer equations for spectral densities of inhomogeneous MHD turbulence", J. Plasma Phys., 44, 103-122. Related online version (cited on 07 September 2005): http://adsabs.harvard.edu/cgi-bin/bib_query?1990JP1Ph. .44..103T. 2.5, 3.1.8, 3.2.1, 9, 13.3

Tu, C.-Y., Marsch, E., 1990b, "Evidence for a 'background' spectrum of solar wind turbulence in the inner heliosphere", J. Geophys. Res., 95, 4337-4341. Related online version (cited on 07 September 2005):

http://adsabs.harvard.edu/cgi-bin/bib_query?1990JGR....95.4337T. 36, 4.2

Tu, C.-Y., Marsch, E., 1991, "A case study of very low cross-helicity fluctuations in the solar wind", Ann. Geophys., 9, 319-332. Related online version (cited on 07 September 2005):

http://adsabs.harvard.edu/cgi-bin/bib_query?1991AnGeo...9..319T. 3.2.1, 3.2.1, 40, 4.2

Tu, C.-Y., Marsch, E., 1993, "A model of solar wind fluctuations with two components:Alfvén waves and convective structures", J. Geophys. Res., 98, 1257-1276. Related online version (cited on 07 September 2005):

http://adsabs.harvard.edu/cgi-bin/bib_query?1993JGR....98.1257T. 3.1.8, 4.1, 9, 9.1

Tu, C.-Y., Marsch, E., 1994, "On the nature of compressive fluctuations in the solar wind", $J$. Geophys. Res., 99, 21 481. Related online version (cited on 07 September 2005):

http://adsabs.harvard.edu/cgi-bin/bib_query?1994JGR ...9921481T. 6.1, 6.1, 6.2, 69

Tu, C.-Y., Marsch, E., 1995a, "MHD structures, waves and turbulence in the solar wind: Observations and theories", Space Sci. Rev., 73, 1-2. Related online version (cited on 07 September 2005):

http://adsabs.harvard.edu/cgi-bin/bib_query?1995SSRv...73...1T. $\quad 1,3.1,17,7$, 3.2.2, 4.1, 4.2, 9, 13.3.2

Tu, C.-Y., Marsch, E., 1995b, "Comment on "Evolution of energy-containing turbulent eddies in the solar wind" by W.H. Matthaeus, S. Oughton, D.H. Pontius, Jr., and Y. Zhou", J. Geophys. Res., 100, 12 323-12 328. Related online version (cited on 07 September 2005):

http://adsabs.harvard.edu/cgi-bin/bib_query?1995JGR...10012323T. 3.1 .2

Tu, C.-Y., Marsch, E., 1996, "Energy spectrum transfer equations of solar wind turbulence", in Solar Wind Eight, (Eds.) Winterhalter, D., Gosling, J.T., Habbal, S.R., Kurth, W.S., Neugebauer, M., Proceedings of the Eighth International Solar Wind Conference, Dana Point, CA 1995, vol. 382 of AIP Conference Proceedings, pp. 233-238, American Institute of Physics, Woodbury, U.S.A. $\quad 2.5$

Tu, C.-Y., Pu, Z.-Y., Wei, F.-S., 1984, "The power spectrum of interplanetary Alfvénic fluctuations Derivation of the governing equation and its solution", J. Geophys. Res., 89, 9695-9702. Related online version (cited on 07 September 2005):

http://adsabs.harvard.edu/cgi-bin/bib_query?1984JGR. . . 89.9695T. 3.1.2, 60, 8.1

Tu, C.-Y., Freeman, J.W., Lopez, R.E., 1989a, "The proton temperature and the total hourly variance of the magnetic field components in different solar wind speed regions", Solar Phys., 119, 197-206. Related online version (cited on 07 September 2005): http://adsabs.harvard.edu/cgi-bin/bib_query?1989SoPh..119...197T. 3.1 .3 
Tu, C.-Y., Marsch, E., Thieme, K.M., 1989b, "Basic properties of solar wind MHD turbulence near 0.3 AU analyzed by means of Elsaesser variables", J. Geophys. Res., 94, 11739-11 759. Related online version (cited on 07 September 2005):

http://adsabs.harvard.edu/cgi-bin/bib_query?1989JGR . . . 9411739T. 3.2, 3.2.2, 3.2.2, $3.2 .2,4.1,5.1$

Tu, C.-Y., Roberts, D.A., Goldstein, M.L., 1989c, "Spectral evolution and cascade constant of solar wind Alfvénic turbulence", J. Geophys. Res., 94, 13 575-13 578. Related online version (cited on 07 September 2005):

http://adsabs.harvard.edu/cgi-bin/bib_query?1989JGR. . .9413575T. 7, 13.3

Tu, C.-Y., Marsch, E., Rosenbauer, H., 1990, "The dependence of MHD turbulence spectra on the inner solar wind stream structure near solar minimum", Geophys. Res. Lett., 17, 283-286. Related online version (cited on 07 September 2005):

http://adsabs.harvard.edu/cgi-bin/bib_query?1990GeoRL..17..283T. 3.2.1, 35

Tu, C.-Y., Marsch, E., Rosenbauer, H., 1991, "Temperature fluctuation spectra in the inner solar wind", Ann. Geophys., 9, 748-753. Related online version (cited on 07 September 2005): http://adsabs.harvard.edu/cgi-bin/bib_query?1991AnGeo...9.748T. 6,64

Tu, C.-Y., Marsch, E., Rosenbauer, H., 1996, "An extended structure-function model and its application to the analysis of solar wind intermittency properties", Ann. Geophys., 14, 270-285. Related online version (cited on 07 September 2005):

http://adsabs.harvard.edu/cgi-bin/bib_query?1996AnGeo..14..270T. 8.1, 8.1, 9.1

Umeki, H., Terasawa, T., 1992, "Decay instability of incoherent Alfvén waves in the solar wind", J. Geophys. Res., 97, 3113-3119. Related online version (cited on 07 September 2005): http://adsabs.harvard.edu/cgi-bin/bib_query?1992JGR . . .97.3113U. 5.2

Van Dyke, M., 1982, An Album of Fluid Motion, The Parabolic Press, Stanford, U.S.A. 4

Vellante, M., Lazarus, A.J., 1987, "An analysis of solar wind fluctuations between 1 and 10 AU", J. Geophys. Res., 92, 9893-9900. Related online version (cited on 07 September 2005): http://adsabs.harvard.edu/cgi-bin/bib_query?1987JGR . . .92.9893V. 6.1

Velli, M., Grappin, R., Mangeney, A., 1989, "Turbulent cascade of incompressible unidirectional Alfvén waves in the interplanetary medium", Phys. Rev. Lett., 63, 1807-1810. Related online version (cited on 07 September 2005):

http://adsabs.harvard.edu/cgi-bin/bib_query?1989PhRvL . 63.1807V. 3.2.2, 5

Velli, M., Grappin, R., Mangeney, A., 1990, "Solar wind expansion effects on the evolution of hydromagnetic turbulence in the interplanetary medium", Computer Phys. Commun., 59, 153162. Related online version (cited on 07 September 2005):

http://adsabs.harvard.edu/cgi-bin/bib_query?1990CoPhC..59..153V. 3.2.2, 5

Veltri, P., 1980, "An observational picture of solar-wind MHD turbulence", Nuovo Cimento C, 3, 45-55. 7

Veltri, P., Mangeney, A., 1999a, "Scaling laws and intermittent structures in solar wind MHD turbulence", in Solar Wind Nine, (Eds.) Habbal, S.R., Hollweg, J.V., Isenberg, P.A., Proceedings of the Ninth International Solar Wind Conference, Nantucket, Massachusetts, October, 1998, vol. 471 of AIP Conference Proceedings, pp. 543-546, American Institute of Physics, Melville, U.S.A. 9

Living Reviews in Solar Physics

http: //www. livingreviews . org/lrsp-2005-4 
Veltri, P., Mangeney, A., 1999b, "Scaling laws and intermittentnstructures in solar wind MHD turbulence", in Solar Wind Nine, (Eds.) Habbal, S.R., Hollweg, J.V., Isenberg, P.A., Proceedings of the Ninth International Solar Wind Conference, Nantucket, Massachusetts, October, 1998, vol. 471 of AIP Conference Proceedings, pp. 543-546, American Institute of Physics, Woodbury, U.S.A. 14

Veltri, P., Mangeney, A., Dobrowolny, M., 1982, "Cross-helicity effects in anisotropic MHD turbulence", Nuovo Cimento B, 68, 235-251. Related online version (cited on 07 September 2005): http://adsabs.harvard.edu/cgi-bin/bib_query?1982NCimB. .68..235V. 3.1.4, 3.2

Veltri, P., Malara, F., Primavera, L., 1992, "Correlation, anisotropy and compressibility of low frequency fluctuations in solar wind", in Solar Wind Seven, (Eds.) Marsch, E., Schwenn, R., Proceedings of the 3rd COSPAR Colloquium held in Goslar, Germany, 16-20 September 1991, vol. 3 of COSPAR Colloquia Series, pp. 423-428, Pergamon Press, Oxford, U.K.; New York, U.S.A. $\quad 5.1$

Viñas, A.F., Goldstein, M.L., 1991, "Parametric instabilities of circularly polarized large-amplitude dispersive Alfvén waves: Excitation of obliquely-propagating daughter and side-band waves", $J$. Plasma Phys., 46, 129.5 .1

Wanner, W., Wibberenz, G., 1993, "A study of the propagation of solar energetic protons in the inner heliosphere", J. Geophys. Res., 98, 3513-3528. Related online version (cited on 07 September 2005):

http://adsabs.harvard.edu/cgi-bin/bib_query?1993JGR....98.3513W. 3.1.5

Yaglom, A.M., 1949, "O lokalnoi strukture polya temperatur v turbulentnom potoke", Dokl. Akad. Nauk. SSSR, 69, 743-746. 2.10

Zank, G.P., Matthaeus, W.H., 1990, "Nearly incompressible hydrodynamics and heat conduction", Phys. Rev. Lett., 64, 1243-1246. Related online version (cited on 07 September 2005): http://adsabs.harvard.edu/cgi-bin/bib_query?1990PhRvL. .64.1243Z. 6.1

Zank, G.P., Matthaeus, W.H., 1991, "The equations of nearly incompressible fluids. I - Hydrodynamics, turbulence, and waves", Phys. Fluids, 3, 69-82. Related online version (cited on 07 September 2005):

http://adsabs.harvard.edu/cgi-bin/bib_query?1991PhFl_..3..667. 6.1, 6.2

Zank, G.P., Matthaeus, W.H., 1992, "Waves and turbulence in the solar wind", J. Geophys. Res., 97, 17 189-17 194. Related online version (cited on 07 September 2005): http://adsabs.harvard.edu/cgi-bin/bib_query?1992JGR . . .9717189Z. 3.1.5, 3.1.5, 6.1

Zank, G.P., Matthaeus, W.H., 1993, "Nearly incompressible fluids. II - Magnetohydrodynamics, turbulence, and waves", Phys. Fluids, 5, 257-273. Related online version (cited on 07 September 2005): http://adsabs.harvard.edu/cgi-bin/bib_query?1993PhFl_...5..257Z. 6.2

Zank, G.P., Matthaeus, W.H., Klein, L.W., 1990, "Temperature and density anti-correlations in solar wind fluctuations", Geophys. Res. Lett., 17, 1239-1242. Related online version (cited on 07 September 2005):

http://adsabs.harvard.edu/cgi-bin/bib_query?1990GeoRL..17.1239Z. 6.1

Zhou, Y., Matthaeus, W.H., 1989, "Non-WKB evolution of solar wind fluctuations: A turbulence modeling approach", Geophys. Res. Lett., 16, 755-758. Related online version (cited on 07 September 2005):

http://adsabs.harvard.edu/cgi-bin/bib_query?1989GeoRL..16. .755Z. 3.2 
Zhou, Y., Matthaeus, W.H., 1990, "Transport and turbulence modeling of solar wind fluctuations", J. Geophys. Res., 95, 10 291-10311. Related online version (cited on 07 September 2005): http://adsabs.harvard.edu/cgi-bin/bib_query?1990JGR . .. 9510291Z. 2.5

Zweben, S.J., Menyuk, C.R., Taylor, R.J., 1979, "Small-Scale Magnetic Fluctuations Inside the Macrotor Tokamak", Phys. Rev. Lett., 42, 1720. Related online version (cited on 07 September 2005):

http://adsabs.harvard.edu/cgi-bin/bib_query?1979PhRvL. .42.1720Z. 3.1.5 